\title{
Simplified Molecular Input Line Entry System-based descriptors in QSAR modelling for HIV-protease inhibitors
}

\author{
Md Ataul Islam, Tahir S. Pillay*, \# \\ Department of Chemical Pathology, Faculty of Health Sciences, University of Pretoria and National Health \\ Laboratory Service Tshwane Academic Division, Pretoria, South Africa. \\ ${ }^{\#}$ Division of Chemical Pathology, Faculty of Health Sciences, University of Cape Town South Africa
}
"Correspondence should be addressed to T.S. Pillay, Department of Chemical Pathology, Faculty of Health Sciences, University of Pretoria, Private Bag X323, Arcadia, Pretoria, 0007
Email: tspillay@gmail.com
Phone: $+27-123192114$
Fax: $+27-123283600$

\section{Highlights}

- QSAR models were developed on HIV-protease inhibitors.

- Descriptors were generated from SMILES-based structures.

- Monte Carlo algorithm was used to correlate the biological activity with descriptors.

- Models were developed with- and without considering influence of inhibitory activity in chemical structures.

- Presence of rings in the molecules was found to be important for inhibition of HIVprotease.

\begin{abstract}
Simplified Molecular Input Line Entry System (SMILES) descriptor based quantitative structure-activity relationship (QSAR) study was performed on a set of HIV-protease inhibitors to explore the structural functionalities for inhibition of the HIV-protease. For this purpose a set of HIV-inhibitors was collected from the literature along with their inhibitory constants. Monte Carlo optimization-based CORAL software was used for QSAR model development. Firstly, the dataset was divided into three random splits and secondly each split was divided into training, calibration, test and validation sets. Training set was used for model development whereas the rest of the sets was used to assess the quality of the developed models. QSAR models were developed with and without considering the influence of cyclic rings towards the inhibitory activity. Statistical quality of QSAR models developed from all splits were very good and fulfilled the criteria. The values of $R^{2}, Q^{2}, s, R_{\text {pred }}^{2}$ and $r_{m}^{2}$ explained that selected models are robust in nature and efficient enough to predict the inhibitory activity of the molecules outside of the training set. Statistical parameters also suggested that the presence of cyclic rings have a crucial impact on inhibitory activity. The
\end{abstract}


molecular fragments were found to be important for the increase or decrease of the inhibitory activity which explained that models have mechanistic interpretation. This ligand-based QSAR study can provide clear directions to design and modulate potential HIV-protease inhibitors.

Keywords: HIV-protease; Descriptors; QSAR; Monte Carlo method, SMILES; CORAL software

\section{Introduction}

The human immunodeficiency virus (HIV-1) is a retrovirus which affects the immune system of the body leaving the victim vulnerable to opportunistic infections, malignancies and neurological disorder with progression of the HIV infection. The acquired immunodeficiency syndrome (AIDS) is the most advanced stage of HIV infection, an epidemic disease with significant health challenges worldwide. Since HIV was identified more than three decades ago, it has spread to almost all parts of the world[1,2]. Owing to its rapid spread it has become a serious global threat and to date, there is no curative treatment for this fatal disease. According to the World Health Organization (WHO), a total of 37.20 million people are living with AIDS and 1.20 million people died in 2014 alone. The HIV$1[1,3-5]$ virus shows extensive and dynamic genetic diversity[6] which has implications for the understanding of viral transmission, pathogenesis and diagnosis, and strongly influences strategies for vaccine development. The HIV-1 protease receptor (HIV-1 PR) is a key therapeutic target for the development of anti-HIV inhibitors[7] for the treatment of AIDS as it plays an important role in the maturation and replication of the virus. HIV-1 PR is comprised of a homodimeric $\mathrm{C}-2$ symmetric structure and one catalytic aspartic residue contributed by each monomer along with threonine and glycine residues which are flexible and a flap that favours the binding of substrate and inhibitors. Highly active antiretroviral therapy (HAART), protease inhibitors (PIs) and reverse-transcriptase inhibitors are widely used for the treatment of HIV -infection worldwide and have resulted in the extraordinary success of HIV/AIDS chemotherapy[8-11]. Rapid development of drug-resistant HIV-1 variants and transmission of these resistant viral strains along with the adverse side effects of currently used HIV-1 PIs, remain critical factors which limit the clinical success of HAART[12-14]. Several research groups globally have developed HIV-1 protease inhibitors which show excellent antiviral profiles[15-21]. A few clinically approved HIV-1 protease inhibitors (atazanavir, indivanir, nelfinavir and sequinavir) are available for treatment of 
HIV/AIDS but these are very peptide-like and have poor bio-availability. Consequently, there is a demand to identify new potential PIs with enhanced activity against drug resistant variants and exceptional pharmacokinetic and safety profiles.

The primary objective of the present work was to establish the relationships between the chemical structure and corresponding biological activity through statistical methods in terms of QSAR modelling. QSARs are statistically validated and mathematical relationships between experimental or calculated properties obtained from molecular structures with biological activities. The experimental or calculated properties obtained from chemical structures are the descriptors which characterize specific information of the molecule being studied. The QSAR models provide insights into the essential structural information of the ligands or inhibitors which contribute to biological activity[22]. Descriptors are generally categorised as physico-chemical, topological and electronic, geometric and structural, and simple indicatorparameters. Further, descriptors can also be divided based on dimensionality (0D, 1D, 2D or 3D)[23]. Geometry-based descriptors are generally difficult to calculate and require high computational costs and long computational calculation time. Consequently the conformation-independent descriptors such as 0D, 1D and 2D QSAR/QSPR models based on the constitutional and topological molecular features of compounds have been developed as an alternate approach[24, 25]. In general the QSAR models are developed on molecular graph based descriptors[26-28] but the simplified molecular input-line entry system (SMILES) representation can also be considered for the molecular structure[29-31] which can further be used for molecular descriptor calculations followed by development of QSAR models. The descriptors based on SMILES notation depend both on the molecular structure and the property under analysis irrespective of details from the 3D-molecular geometry[22]. The descriptors generated from SMILES-based molecular structure and further used for development of QSAR models is an attractive direction of research work in the field of the QSAR theory and applications[32-34]. Several research groups have already proven the importance of the methodology, which was capable of developing models with a similar or improved quality to the ones built with descriptors containing thousands of 0D-3D descriptors[35-41].

Therefore the current research work was considered to build QSAR models based on the SMILES notation optimal descriptors using the Monte Carlo method for HIV-protease inhibitors followed by analysis and explanation of involvement of molecular fragments responsible for the inhibition of HIV-protease. 


\section{Materials and methods}

\subsection{Dataset}

In order to develop QSAR models a set of 129 HIV protease inhibitors[42-44] with experimental inhibitory constant $\left(K_{i}\right)$ were collected from literature. The fluorescence resonance energy transfer (FRET) method had been used to determine experimental $K_{i}$ values by the same group of authors. The molecules of the dataset have a wide range of $K_{i}$, from 0.0008 to $237.8 \mathrm{nM}$. All datasets are provided in the Supplementary file (Tables S1 - S3) with their inhibitory activity. The SMILES format of whole dataset was generated for input purposes. The $K i$ values were converted into $p K_{i}$ as $p K_{i}=\log \left[\left(1 / K_{i}\right) \mathrm{x} 1000\right]$ and considered as the endpoint of the QSAR. The whole dataset was randomly divided into three splits (Splits 1 to 3) and further each split was arbitrarily distributed into training, calibration, test and validation sets. Each of the set has specific role in QSAR formulation. The training set was used for model development and calibration and test sets were used to check the predictive ability of developed model. The validation set was used for final estimation of the model using those compounds that were invisible during model formulationi.e. no information from the validation set was involved in the model generation.

\subsection{Optimal descriptors}

The molecular structures were converted into SMILES format which is one of the convenient representations of chemical compounds. The SMILES format can be used to select optimal molecular descriptors which are mathematical functions of so-called correlation weights (CW) that is "Descriptors of Correlation Weights" (DCW). The Monte Carlo algorithm was used to calculate the DCW. Firstly, the DCW were calculated without considering the influence of the cyclic rings as per following equation (1).

$$
\begin{aligned}
D C W_{1}\left(S M I L E S, T, N_{e p o c h}\right) & =\alpha \sum C W\left(S_{k}\right)+\beta \sum C W\left(S S_{k}\right)+\gamma \sum C W\left(S S S_{k}\right)+\mathrm{x} \cdot C W(N O S P) \\
& +\mathrm{y} \cdot C W(H A L O)+z \cdot C W(B O N D)+t \cdot C W(P A I R)
\end{aligned}
$$

Where, $\mathrm{T}$ is the threshold which defines as coefficient for classifying various molecular features extracted from SMILES into two classes: a) active, in which CW is involved in the modelling process and b) rare, in which $\mathrm{CW}$ is not involved in the modelling process. The $\mathrm{N}_{\text {epoch }}$ is the number of epochs used in the Monte Carlo optimization that provides the best statistical results of the calibration set. The $S_{k}$ represents one or two symbols from SMILES which cannot be inspected separately. On combination of two or three $\mathrm{S}_{\mathrm{k}}$ the $\mathrm{SS}_{\mathrm{k}}$ and $\mathrm{SSS}_{\mathrm{k}}$ are 
formed respectively. NOSP, HALO, BOND and PAIR are different descriptors based on presence of absence of different chemical elements and bonds. NOSP refers to nitrogen, oxygen, sulphur and phosphorus; HALO indicates halogen atoms such as fluorine, chlorine and bromine; BOND indicates double (=), triple (\#) or stereochemical bonds (@ or @ @); and PAIR refers to the probable grouping of pair atoms and/or SMILES attributes (for example double, triple, and stereochemical bonds) that takes place in the structure together. $\alpha$, $\beta, \gamma, \mathrm{x}, \mathrm{y}$ and $\mathrm{t}$ are discrete coefficient with values 0 and 1. Details of the above descriptors with example are explained by Worachartcheewan et al.[39].

In the case of the second version of optimal descriptors calculation, in addition to the equation (1) the influence of cyclic rings on inhibitory activity were also considered and the equation is given below (2).

$$
\begin{aligned}
D C W_{2}\left(S M I L E S, T, N_{\text {epoch }}\right) & =\alpha \sum C W\left(S_{k}\right)+\beta \sum C W\left(S S_{k}\right)+\gamma \sum C W\left(S S S_{k}\right)+\mathrm{x} \cdot C W(N O S P) \\
& +\mathrm{y} \cdot C W(H A L O)+z \cdot C W(B O N D)+t \cdot C W(P A I R) C W(\mathrm{C} 3)+C W(C 4) \\
& +C W(C 5)+C W(C 6)+C W(C 7)
\end{aligned}
$$

Where, C3, C4, C5, C6 and C7 are three-membered cycles, four-membered cycles, fivemembered cycles, six-membered cycles and seven-membered cycles. Details of such descriptors are explained elsewhere [45]. The purpose of optimization is to obtain the maximal correlation coefficient between optimal descriptors and the end point of the training set.

Monte Carlo method was used to calculate the $\mathrm{CW}$ which gives the best statistical results for the test set. In order to search the preferable threshold $\left(\mathrm{T}^{*}\right)$ and preferable number of epochs $\left(\mathrm{N}^{*}\right)$, range of $\mathrm{T}$ and $\mathrm{N}_{\text {epoch }}$ were selected from 1 to 10 and 1 to 20 respectively. The best $\left(\mathrm{N}^{*}\right.$, $\mathrm{T}^{*}$ ) were selected from each split based on best statistical results. The preferable statistics of the calibration set makes it possible to calculate the endpoint value using numerical values of correlation weights from the training set as follows:

$$
\text { Endpoint }=C_{0}+C_{1} \times \text { DCW }\left(\text { SMILES, } \mathrm{T}, \mathrm{N}_{\text {epoch }}\right)
$$

Where, endpoint is the inhibitory constant and, $\mathrm{C}_{0}$ and $\mathrm{C}_{1}$ are constant. The external validation set which was not involved for model building was used to verify the predictive potential of the model. 


\subsection{Validation}

Validation of any statistical model is an essential and crucial step to verify the predictive ability and reliability of the model. Any QSAR model can be validated by means of a) internal validation using training set compounds; b) external validation using test compounds; and c) Y-scrambling or randomization of data. Several studies[34, 35, 37, 38, 41] used these validation methodologies on SMILES notation optimal descriptor based QSAR models. In this respect the cross-validated correlation coefficient $\left(Q^{2}\right)$ and error of estimation $(s)$ were calculated based on predicted activity of training compounds. High $Q^{2}(>0.5)$ and low $s$ explained better predictive ability of the model[46]. Furthermore, in order to confirm the good predictive ability of the training set compounds, the modified $r^{2}\left(r_{m(L O O)}^{2}\right)$ developed by Roy et al.[47, 48] the $r_{m(L O O)}^{2}$ was calculated which is the measure of the degree of deviation of the predicted activity from the observed ones. It was reported that model may be considered with $r_{m(L O O)}^{2}>0.5$. For calibration, test and external validation set compounds the $R_{\text {pred }}^{2}$ (correlation coefficient) was also calculate to judge the productiveness of developed model. It is reported that models with $R_{\text {pred }}^{2} \geq 0.5$ can be considered for further evaluation. The value of $R_{\text {pred }}^{2}$ concerns the mean experimental activity of the training set compounds. Consequently good statistical value of $R_{\text {pred }}^{2}$ might be found for molecules with a varied range of activity values, but this does not confirm that the predicted activity values are well correlated with observed activity. However, if a high correlation is preserved, there might be a substantial numerical difference between the two values. In order to better indicate the predictive ability of the model, modified $r^{2}\left[r_{m(t e s t)}^{2}\right][49,50]$ values were calculated (threshold value $=0.5$ ).In order to verify the chance correlation $\mathbf{Y}$-scrambling described by Ojha and Roy[51] was also performed in which ten probes of calculation were carried out. In one probe of calculation, $\mathrm{X}$ and $\mathrm{Y}$ represent the vectors of experiment and the vector of prediction. First of all exchange of random $\mathrm{N} 1$ and random $\mathrm{N} 2$ from row $\mathrm{X}$ ( $\mathrm{Y}$ is not modified) were performed thousand times. Further, from above probes the $R_{(X, Y)}^{2}$ was calculated and represented as $R_{r}^{2}$. The ${ }^{C} R_{p}^{2}$ was finally calculated according to the equation (3).

${ }^{C} R_{p}^{2}=R \times\left(R^{2}-R_{r}^{2}\right)^{1 / 2}$

Where $R^{2}$ and $R^{2}{ }_{r}$ were utilized from the non-randomized and randomized model respectively. For acceptance of QSAR model the threshold value of ${ }^{C} R_{p}^{2}$ should be greater than 0.5 . 


\section{Results and discussion}

In the current research work, HIV-protease inhibition constant activity $\left(K_{i}\right)$ was used as a function of molecular features extracted from SMILES-based attributes. A set of 129 HIVprotease inhibitors were modelled in online freely available CORAL software (http://www.insilico.eu/coral/) which is based on the Monte Carlo optimization technique. In this technique molecular description in the form of SMILES was used to develop well predictive and statistically validated QSAR models and further QSAR models were used to predict the endpoint data that is the $p K_{i}$ values. Two approaches were considered to develop QSAR models viz. with and without considering influence of cyclic rings of the molecules in inhibitory activity. In order to identify the optimal $\mathrm{T}$ and $\mathrm{N}_{\text {epoch }}\left(\mathrm{T}^{*}\right.$ and $\mathrm{N}^{*}{ }_{\text {epoch }}$ ) for Monte Carlo optimization the training set molecules were used to develop models using threshold values in the range of 1 to 10 and the number of epochs ranging from 1 to 20 (Figure 1 ) for each splits. Finally the $\mathrm{T}^{*}$ and $\mathrm{N}^{*}$ were chosen based on best statistical results and further used for constructing the QSAR models. In case of without considering influence of rings on activity, the best $\left(\mathrm{T}^{*}, \mathrm{~N}^{*}\right)$ for splits 1,2 and 3 were found to be $(1,17),(1,12)$ and $(1,19)$ respectively. On the other hand, the optimal $\left(\mathrm{T}^{*}, \mathrm{~N}^{*}\right)$ were $(1,19),(1,18)$ and $(1,12)$ for splits 1, 2 and 3 respectively by considering the influence of rings on the inhibitory activity. The whole data set was divided into four groups i.e. training, calibration, test and validation sets in each split. The training set was used for model generation while rest of the sets were used for evaluating the predictive performance of generated models.

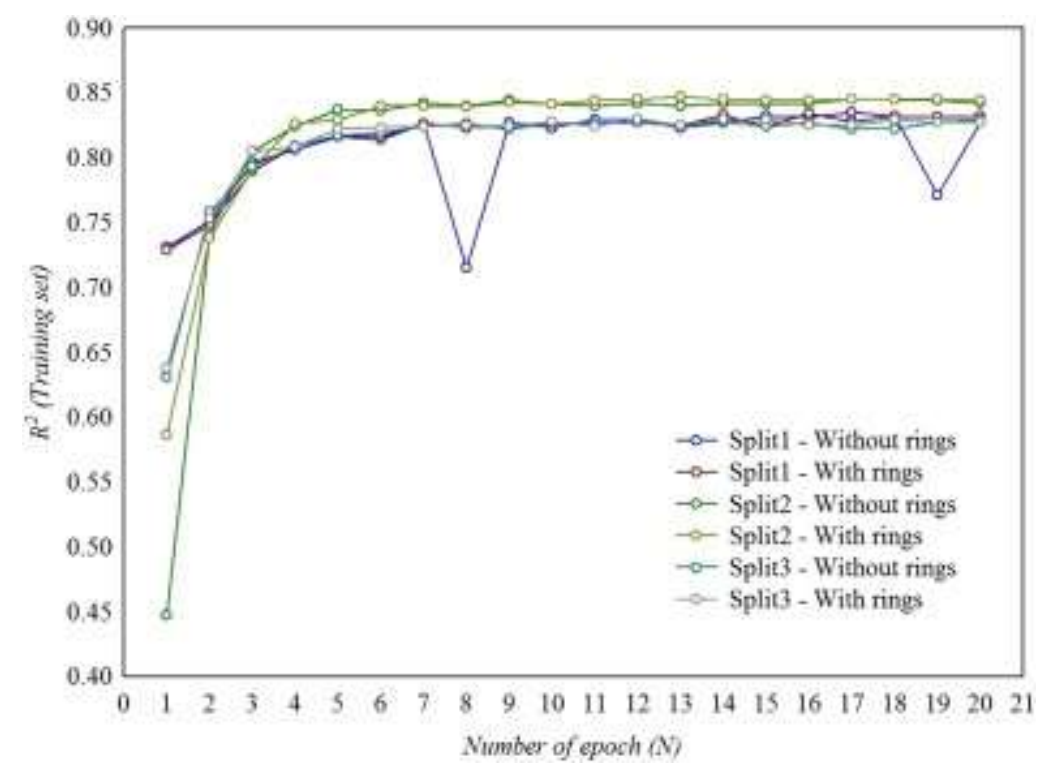

Figure 1: Number of epoch (N) vs. correlation coefficient. 


\section{Split 1:}

Without considering influence of various cyclic rings:

$$
p K_{i}=0.0001( \pm 0.0255)+0.0580( \pm 0.0005) \times D C W(1,17) \quad(\text { Model 1) }
$$

Training set: $\quad n=70 ; R^{2}=0.837 ; s=0.448 ; F=331 ; Q^{2}=0.831 ; s_{p}=0.455 ; r_{m}^{2}=$

$\begin{array}{ll} & 0.830 ; \Delta r^{2}{ }_{m}=0.149 \\ \text { Calibration set: } & n=20 ; R^{2}=0.952 ; s=0.312 ; F=332 ; R^{2}{ }_{\text {pred }}=0.948 ; r_{m}^{2}=0.949 ; \Delta r_{m}^{2}\end{array}$ $=0.040$

Test set: $\quad n=21 ; R^{2}=0.799 ; s=0.561, F=72 ; R_{\text {pred }}^{2}=0.769 ; r_{m}^{2}=0.686 ; \Delta r_{m}^{2}$ $=0.043$

External set: $\quad n=18 ; R^{2}=0.794 ; s=0.677, F=82 ; R_{\text {pred }}^{2}=0.781 ; r_{m}^{2}=0.791 ; \Delta r_{m}^{2}$ $=0.043$

Considering influence of various cyclic rings:

$p K_{i}=-0.0011( \pm 0.0267)+0.0461( \pm 0.0004) \times D C W(1,19)$

(Model 2)

Training set: $\quad n=70 ; R^{2}=0.811 ; s=0.482, F=346 ; Q^{2}=0.807, s_{p}=0.447 ; r_{m}^{2}=$ $0.836 ; \Delta r_{m}^{2}=0.144$

Calibration set: $\quad n=20 ; R^{2}=0.950 ; s=0.315 ; F=382 ; R_{\text {pred }}^{2}=0.943 ; r_{m}^{2}=0.953$; $\Delta r_{m}^{2}=0.036$

Test set: $\quad n=21 ; R^{2}=0.806 ; s=0.553, F=85 ; R_{\text {pred }}^{2}=0.800 ; r_{m}^{2}=0.690 ; \Delta r_{m}^{2}$ $=0.103$

External set: $\quad n=18 ; R^{2}=0.752 ; s=0.738, F=51 ; R_{\text {pred }}^{2}=0.729 ; r_{m}^{2}=0.630 ; \Delta r_{m}^{2}$ $=0.080$

\section{Split 2:}

Without considering influence of various cyclic rings:

$p K_{i}=-0.0035( \pm 0.0196)+0.0485( \pm 0.0003) \times D C W(1,12)$

Training set:

$n=72 ; R^{2}=0.843 ; s=0.454, F=376 ; Q^{2}=0.830 ; s_{p}=0.451 ; r_{m}^{2}=$ $0.843 ; \Delta r_{m}^{2}=0.138$

Calibration set: $\quad n=20 ; R^{2}=0.947 ; s=0.304 ; F=324 ; R_{\text {pred }}^{2}=0.943 ; r_{m}^{2}=0.946$; $\Delta r_{m}^{2}=0.046$

Test set:

$n=19 ; R^{2}=0.781 ; s=0.676 ; F=61 ; R_{\text {pred }}^{2}=0.778 ; r_{m}^{2}=0.694 ; \Delta r_{m}^{2}$

External set: $=0.005$

$n=18 ; R^{2}=0.740 ; s=0.644, F=45 ; R_{\text {pred }}^{2}=0.736 ; r_{m}^{2}=0.531 ; \Delta r_{m}^{2}$ $=0.209$ 
Considering influence of various cyclic rings:

$$
p K_{i}=0.0053( \pm 0.0196)+0.0652( \pm 0.0004) \times D C W(1,18)
$$

Training set: $n=72 ; R^{2}=0.841 ; s=0.457 ; F=370 ; Q^{2}=0.828, s_{p}=0.454 ; r_{m}^{2}=$ $0.841 ; \Delta r_{m}^{2}=0.130$

Calibration set: $\quad n=20 ; R^{2}=0.941 ; s=0.323 ; F=286 ; R_{\text {pred }}^{2}=0.937 ; r_{m}^{2}=0.939$; $\Delta r_{m}^{2}=0.107$

Test set:

$n=19 ; R^{2}=0.784 ; s=0.617 ; F=89 ; R_{\text {pred }}^{2}=0.779 ; r_{m}^{2}=0.827 ; \Delta r_{m}^{2}$

External set: $=0.107$

\section{Split 3:}

$n=18 ; R^{2}=0.753 ; s=0.624 ; F=45 ; R_{\text {pred }}^{2}=0.751 ; r_{m}^{2}=0.538 ; \Delta r_{m}^{2}=$ 0.203

Without considering influence of various cyclic rings:

$$
p K_{i}=-0.00001( \pm 0.0204)+0.0463( \pm 0.0003) \times D C W(1,19)
$$

Training set: $n=75 ; R^{2}=0.829 ; s=0.491 ; F=355 ; Q^{2}=0.826 ; s_{p}=0.487 ; r_{m}^{2}=$ $0.829 ; \Delta r^{2}{ }_{m}=0.148$

Calibration set: $\quad n=20 ; R^{2}=0.945 ; s=0.335 ; F=311 ; R_{\text {pred }}^{2}=0.943 ; r_{m}^{2}=0.945$; $\Delta r_{m}^{2}=0.048$

Test set:

$n=19 ; R^{2}=0.804 ; s=0.592 ; F=70 ; R_{\text {pred }}^{2}=0.811 ; r_{m}^{2}=0.614 ; \Delta r_{m}^{2}$

External set: $=0.168$

$n=15 ; R^{2}=0.881 ; s=0.381 ; F=96 ; R_{\text {pred }}^{2}=0.907 ; r_{m}^{2}=0.791 ; \Delta r_{m}^{2}=$ 0.075

Considering influence of various cyclic rings:

$$
p K_{i}=-0.00003( \pm 0.0211)+0.0393( \pm 0.0002) \times D C W(1,12)
$$

Training set:

$$
n=75 ; R^{2}=0.830 ; s=0.489 ; F=357 ; Q^{2}=0.827, s_{p}=0.486 ; r_{m}^{2}=
$$
$0.830 ; \Delta r^{2}=0.148$

Calibration set: $n=20 ; R^{2}=0.945 ; s=0.335 ; F=311 ; R_{\text {pred }}^{2}=0.944 ; r_{m}^{2}=0.945$;

Test set: $\Delta r_{m}^{2}=0.048$

External set: $n=19 ; R^{2}=0.773 ; s=0.615 ; F=58 ; R^{2}$ red $=0.796 ; r_{m}^{2}=0.602 ; \Delta r_{m}^{2}$ $=0.159$

$$
\begin{aligned}
& n=15 ; R^{2}=0.915 ; s=0.311 ; F=140 ; R^{2}{ }_{\text {red }}=0.938 ; r_{m}^{2}=0.883 ; \\
& \Delta r_{m}^{2}=0.012
\end{aligned}
$$

Models 1, 3 and 5 were developed from splits 1, 2 and 3 respectively without considering any influence of rings on the inhibitory activity of the training set molecules. On the other side, Models 2, 3 and 4 were generated with involvement of the influence of rings on the inhibitory activity of training set molecules of splits 1,2 and 3 respectively. All models were developed 
using best Monte Carlo optimization runs and also according to Organisation for Economic and Co-operation (OECD) principles[52].

Table 1: Statistical parameters of training, calibration, test and validation sets of Models 1 to 6

\begin{tabular}{|c|c|c|c|c|c|c|c|c|c|c|c|}
\hline Model & set & $n$ & $R^{2}$ & $s$ & $F$ & $Q^{2}$ & ${ }^{C} R_{p}^{2}$ & $\boldsymbol{R}_{\text {pred }}^{2}$ & $R^{2}-Q^{2}$ & $r_{m}^{2}$ & $\Delta r_{m}^{2}$ \\
\hline \multirow{4}{*}{ Model 1} & Training & 70 & 0.837 & 0.448 & 331 & 0.831 & 0.823 & - & 0.006 & 0.830 & 0.149 \\
\hline & Calibration & 20 & 0.952 & 0.312 & 332 & - & 0.907 & 0.948 & - & 0.949 & 0.040 \\
\hline & Test & 21 & 0.799 & 0.561 & 72 & - & 0.782 & 0.769 & - & 0.686 & 0.043 \\
\hline & Validation & 18 & 0.794 & 0.677 & 82 & - & - & 0.781 & - & 0.791 & 0.043 \\
\hline \multirow{4}{*}{ Model 2} & Training & 70 & 0.811 & 0.482 & 346 & 0.807 & 0.824 & - & 0.004 & 0.836 & 0.144 \\
\hline & Calibration & 20 & 0.950 & 0.315 & 382 & - & 0.937 & 0.943 & - & 0.953 & 0.036 \\
\hline & Test & 21 & 0.806 & 0.553 & 85 & - & 0.796 & 0.800 & - & 0.690 & 0.103 \\
\hline & Validation & 18 & 0.752 & 0.738 & 51 & - & - & 0.729 & - & 0.630 & 0.080 \\
\hline \multirow{4}{*}{ Model 3} & Training & 72 & 0.843 & 0.454 & 376 & 0.830 & 0.830 & - & 0.013 & 0.843 & 0.138 \\
\hline & Calibration & 20 & 0.947 & 0.304 & 324 & - & 0.903 & 0.943 & - & 0.946 & 0.046 \\
\hline & Test & 19 & 0.781 & 0.676 & 61 & - & 0.766 & 0.778 & - & 0.694 & 0.005 \\
\hline & Validation & 18 & 0.740 & 0.644 & 45 & - & - & 0.736 & - & 0.531 & 0.209 \\
\hline \multirow{4}{*}{ Model 4} & Training & 72 & 0.841 & 0.457 & 370 & 0.828 & 0.834 & - & 0.013 & 0.841 & 0.130 \\
\hline & Calibration & 20 & 0.941 & 0.323 & 286 & - & 0.919 & 0.937 & - & 0.939 & 0.107 \\
\hline & Test & 19 & 0.784 & 0.617 & 89 & - & 0.811 & 0.779 & - & 0.827 & 0.107 \\
\hline & Validation & 18 & 0.753 & 0.624 & 45 & - & - & 0.751 & - & 0.538 & 0.203 \\
\hline \multirow{4}{*}{ Model 5} & Training & 75 & 0.829 & 0.491 & 355 & 0.826 & 0.827 & - & 0.003 & 0.829 & 0.148 \\
\hline & Calibration & 20 & 0.945 & 0.335 & 311 & - & 0.923 & 0.943 & - & 0.945 & 0.048 \\
\hline & Test & 19 & 0.804 & 0.592 & 70 & - & 0.787 & 0.811 & - & 0.614 & 0.168 \\
\hline & Validation & 15 & 0.881 & 0.381 & 96 & - & - & 0.907 & - & 0.791 & 0.075 \\
\hline \multirow{4}{*}{ Model 6} & Training & 75 & 0.830 & 0.489 & 357 & 0.827 & 0.825 & - & 0.003 & 0.830 & 0.148 \\
\hline & Calibration & 20 & 0.945 & 0.335 & 311 & - & 0.933 & 0.944 & - & 0.945 & 0.048 \\
\hline & Test & 19 & 0.804 & 0.592 & 70 & - & 0.748 & 0.811 & - & 0.614 & 0.168 \\
\hline & Validation & 15 & 0.881 & 0.381 & 96 & - & - & 0.907 & - & 0.791 & 0.075 \\
\hline
\end{tabular}


The statistical results of Models 1 to $\mathbf{6}$ are depicted in Table 1. Values of Table 1 clearly indicated that all models are statistically reliable and fulfilled the criterion explained by Tropsha et al.[53] and Ojha et al.[48] for a predictive QSAR model such as $R^{2}>0.6, Q^{2}>0.5$, ${ }^{C} R_{p}^{2}>0.5,\left(R^{2}-Q^{2}\right)<0.2, R_{p r e d}^{2}>0.5, r_{m}^{2}>0.5$ and $\Delta r_{m}^{2}<0.1$. Experimental and predicted activities of all sets are delineated in Figure 2 and supplementary materials (Tables S1 - S3). The high $R^{2}, Q^{2}$ and $R_{\text {pred }}^{2}$ values of all models explained close prediction of inhibitory activity to the experimental activity which supports the robustness of the models. The reliability of the developed models were also assessed by the difference between $R^{2}$ and $Q^{2}$ $\left(R^{2}-Q^{2}\right)$ which is a metric that accounts for the fraction of $\mathbf{Y}$-data explained by accumulated chance correlations. It is reported that values of the metric less than 0.2 implies that model

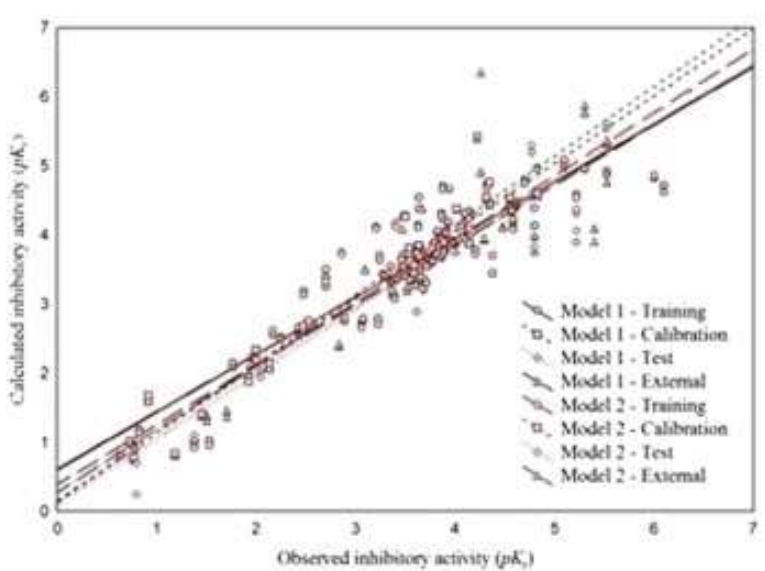

a

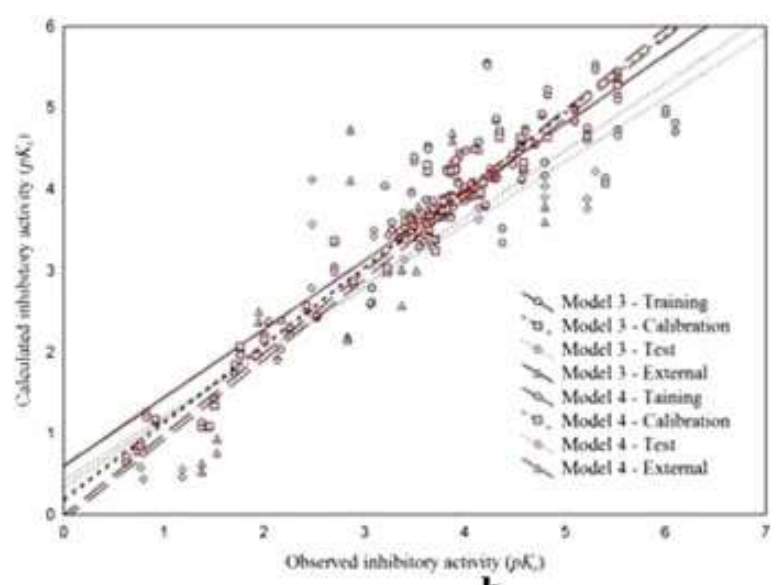

b

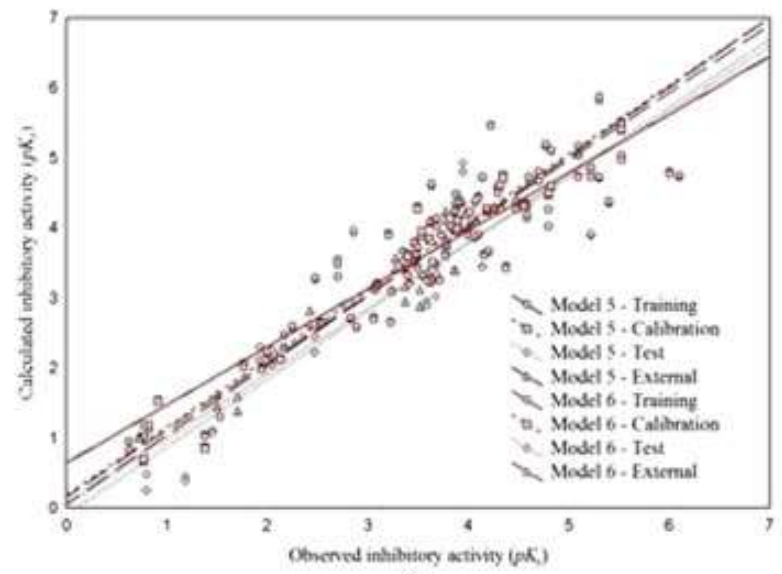

c

Figure 2: Observed and predicted activities as per Models 1 -6. a) Split 1; b) Split 2 and c) Split 3.

developed not by chance[39]. In case of Models 1 to $\mathbf{6}$ the values of the metric lie between 0.130 to 0.003 which is below the criteria mentioned above, therefore models were developed not by chance. Similarly chance correlations were evaluated by the $\mathbf{Y}$-scrambling technique. In the method the $\mathrm{Y}$ value (i.e. $p K_{i}$ ) was randomized and reordered with respect to its associated descriptors. Correspondingly the Y-scrambling was performed for 1000 trials in 
ten separate runs for all three splits. The average value of each run is given in supplementary file (Tables S4 - S6). If the correlation of randomised runs are better than the original then the models may be developed by chance. In the present study it was observed that no randomised run obtained better correlation than the original run in case of Models $\mathbf{1}$ to $\mathbf{6}$. Therefore Models 1 to $\mathbf{6}$ were developed not by chance and they are robust in nature. The correlation values of original and randomized runs are given in supplementary file (Tables S4 - S6).

Comparison of results (Table 1) between models developed by considering (Models 2, 4 and 6) and without considering (Models 1, 3 and 5) influence of the cyclic rings of the molecular scaffolds of training sets on inhibitory activity revealed that the rings have considerable impact on inhibition of HIV-protease as statistical results are better in case of calibration, test and external sets for Models 2, 4 and 6. In the current research, descriptors used were based on SMILES notation that means ignoring the three dimensional molecular structures of dataset which reduce the computation time as well as computation resources. All statistical parameters satisfied the criteria except $\Delta r_{m}^{2}$ for external validation set in Models $\mathbf{3}$ and 4, where it is more than 0.2 . It is noted that if $r_{m}^{2}$ is more than 0.5 then $\Delta r_{m}^{2}$ should preferably less than 0.2. But it is also reported that this is not a rigid limitation[54]. Radar plots of training set molecules of Models 1 to 6 were plotted and delineated in Figure 3. The plots explore the fitness of experimental and calculated inhibitory activity. Substantial overlapping of experimental and calculated $p K_{i}$ values in the radar plots support the idea that the models are efficient enough to predict the biological activity of the molecules of training set molecules. 


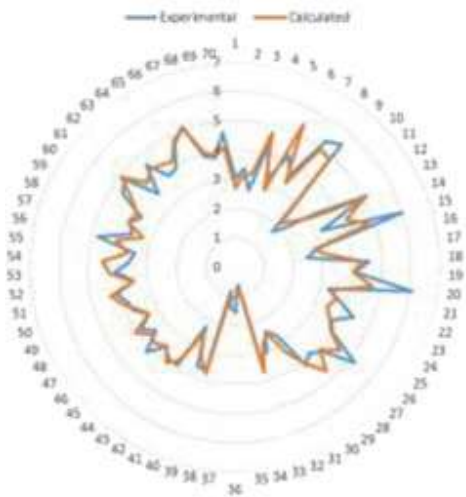

Model 1

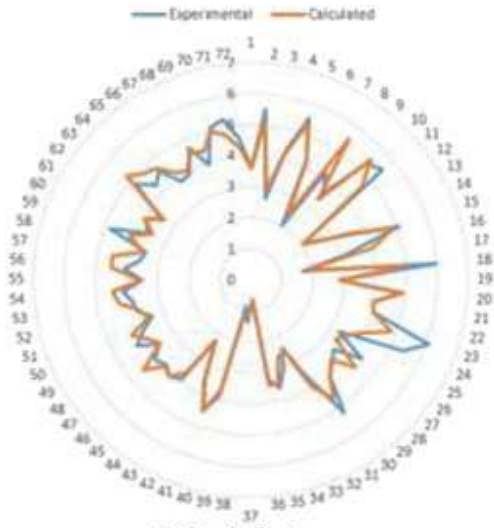

Model 4

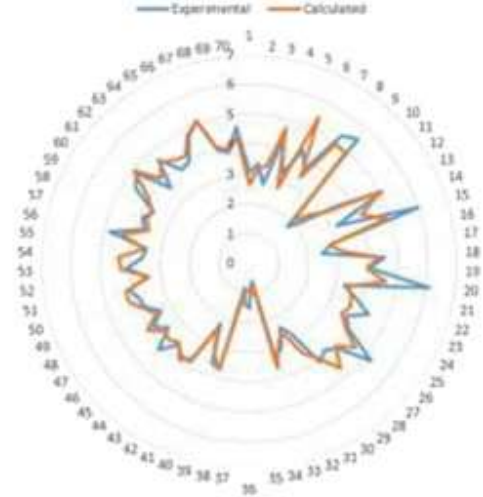

Model 2

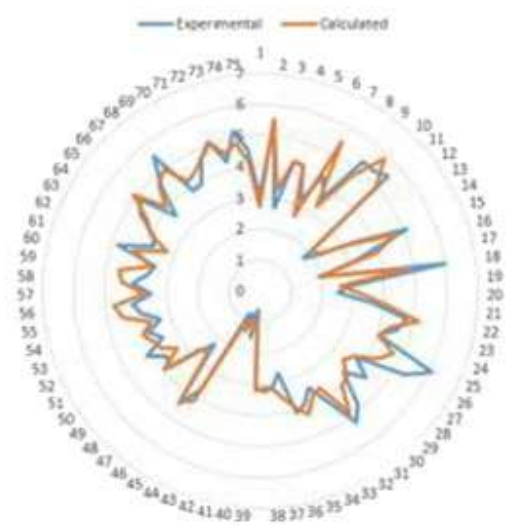

Model 5

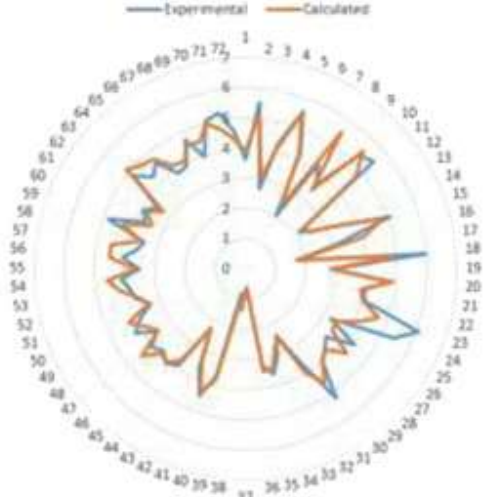

Model 3

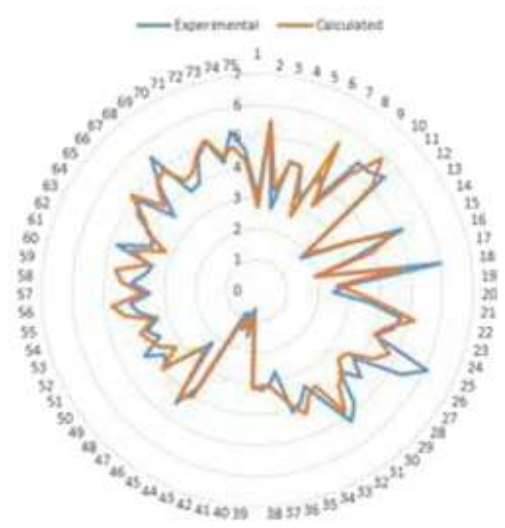

Model 6

Figure 3: Radar plot showing fitness of predicted and actual activity values of training set of Models 1 to 6

The molecular fragments obtained from SMILES were interpreted to explore chemical information analysed using correlation weights from QSAR models. This analysis was performed on the data samples divided into the following categories: a) molecular features with constant positive values of correlation weights i.e. positive correlation weights in all runs, increased for an endpoint; b) molecular features with negative constant value of correlation weights in all runs decreased for an endpoint; c) unstable molecular features which have both positive and negative correlation weights in several runs. The correlation weights of molecular features greater than ' 0 ' implies that molecular features have positive influence on inhibitory activity, if it is less than ' 0 ' then molecular features have negative influence on the inhibitory activity. If the correlation weights of molecular features have a mix of greater than and less than ' 0 ' values then the impact of molecular features on the inhibitory activity is undefined.

In this manuscript molecule $\mathbf{H 1}$ was considered to explain the impact of molecular features on $p K_{i}$. It was observe that smiles attributes ' $3 \ldots .$. ' and ' $\mathrm{C} \ldots \ldots 1$ ' have positive influence on $p K_{i}$ in case of Models 3, 4, 5 and 6. The attribute NOSP11100000 (presence of 'N', 'O' and 
' $\mathrm{S}$ ' but absence of 'P') gives negative impact on $p K_{i}$ in case of Models $\mathbf{2}-\mathbf{6}$ but positive for Model 1. The HALO00000000 (absence of halogen atoms) increases the $p K_{i}$ in the case of models developed without considering the influence of the rings (Models 1, 3 and 5), but decreases the $p K_{i}$ in the presence of cyclic rings (Models 2, 4 and 6). BOND10100000 attributes contribute negative impact on all models except Model 6. Decrease of $p K_{i}$ due to presence of oxygen and double bond occurs in all models. Presence of nitrogen atom in case of Models 3 - $\mathbf{6}$ gives an increased $p K_{i}$ value while for Models $\mathbf{1}$ and 2 its involvement decreases the $p K_{i}$ value. The ' $++++\mathrm{N}---\mathrm{S}===$ ' was found to be important for increasing the $p K_{i}$ in the case of the presence of cyclic rings (Models 2, 4 and 6). Another attribute ' $++++\mathrm{S}-$ --B2==' gives negative influence on $p K_{i}$ in for Models $2-\mathbf{5}$, but for Models $\mathbf{1}$ and $\mathbf{6}$ its impact is positive. Presence of chirality with anti-clockwise ('@') gives negative influence for inhibitory activity for Models 1, 2, 4, and 6. Influence of chirality with clockwise ('@@) increases the $p K_{i}$ in all models except Model 2. From the above discussion it is clear that models developed (Models 1 - 6) using the HIV-protease inhibitors with considering the inhibitory constant as endpoints have mechanistic interpretations for deducing how the molecular features can be attributed to appropriate atoms and/or molecular fragments.

The $p K_{i}$ of well-known HIV-protease inhibitors such as atazanavir, indivanir, nelfinavir and sequinavir were calculated as per Models $\mathbf{1}$ to $\mathbf{6}$ and given in Table 2. The calculated activity explained that influence of rings have positive impact inhibitory activity as $p K_{i}$ values were found to be higher in models developed with considering rings (Models 2, 4 and 6) in comparison to models developed without considering the influence of rings in inhibitory activity. Therefore the models developed using atomic and molecular fragments using SMILES notation can successfully predict the inhibitory activity of unknown molecules. Therefore the developed models can be used to design new molecular scaffolds of HIVprotease inhibitors with increased or decreased inhibitory activities. 
Table 2: Calculated inhibitory activity $(p K i)$ of different standard HIV-protease inhibitors based on QSAR models

Molecule Structure $p K_{i}$

Model 1 Model 2 Model 3 Model 4 Model 5 Model 6

Atazanavir

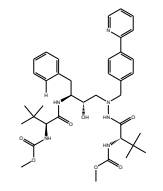

2.324

2.385

2.684

2.873

2.652

2.786

Indivanir

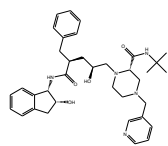

2.358

2.363

2.418

2.571

2.280

2.461

Nelfinavir

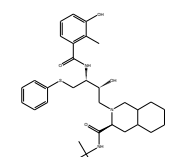

2.510

3.171

2.782

2.791

3.195

3.062

Sequinavir

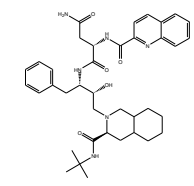

2.524

3.243

3.796

2.650

5.699

3.395

\section{Conclusions}

The predictive QSAR models for HIV-protease inhibitors were developed with inhibitory constant using online CORAL software. Incorporation of the Monte Carlo optimization technique in CORAL software provides an effective and prominent tool to develop good statistical QSAR models. SMILES-based descriptors were used with and without considering the influence of different rings on the inhibitory activity. The predictive performance of all models were tested. The radar plot also explained the well-fitness of experimental and calculated inhibitory activities of training set molecules. The statistical quality of the models explained that cyclic rings influence the inhibition of HIV-protease. All models have mechanistic interpretations in terms of the importance of molecular features on correlation weights for increases or decreases in the inhibitory activity $\left(p K_{i}\right)$. The developed models can be used to for prediction of inhibitory constants for HIV-protease inhibitors without experiments. The predictive nature of all models was validated according to OECD guidelines. Therefore it can be concluded that the data represented indicates that the suggested approach can be applied to design novel and potential HIV protease inhibitors for the wider community of people with HIV. 


\section{Acknowledgement}

MA Islam and TS Pillay were funded by the University of Pretoria Vice Chancellor's postdoctoral fellowship and National Research Foundation (NRF), South Africa Innovation postdoctoral fellowship schemes.

\section{References}

[1] R.C. Gallo, P.S. Sarin, E.P. Gelmann, M. Robert-Guroff, E. Richardson, V.S. Kalyanaraman, D. Mann, G.D. Sidhu, R.E. Stahl, S. Zolla-Pazner, J. Leibowitch, M. Popovic, Isolation of human T-cell leukemia virus in acquired immune deficiency syndrome (AIDS), Science, 220 (1983) 865-867.

[2] N. Chin'ombe, V. Ruhanya, HIV/AIDS vaccines for Africa: scientific opportunities, challenges and strategies, The Pan African medical journal, 20 (2015) 386.

[3] F. Barre-Sinoussi, J.C. Chermann, F. Rey, M.T. Nugeyre, S. Chamaret, J. Gruest, C. Dauguet, C. Axler-Blin, F. Vezinet-Brun, C. Rouzioux, W. Rozenbaum, L. Montagnier, Isolation of a T-lymphotropic retrovirus from a patient at risk for acquired immune deficiency syndrome (AIDS), Science, 220 (1983) 868-871.

[4] M. Popovic, M.G. Sarngadharan, E. Read, R.C. Gallo, Detection, isolation, and continuous production of cytopathic retroviruses (HTLV-III) from patients with AIDS and pre-AIDS, Science, 224 (1984) 497-500.

[5] M.G. Sarngadharan, M. Popovic, L. Bruch, J. Schupbach, R.C. Gallo, Antibodies reactive with human Tlymphotropic retroviruses (HTLV-III) in the serum of patients with AIDS, Science, 224 (1984) 506-508.

[6] J. Hemelaar, E. Gouws, P.D. Ghys, S. Osmanov, Global and regional distribution of HIV-1 genetic subtypes and recombinants in 2004, Aids, 20 (2006) W13-23.

[7] A. Wlodawer, M. Miller, M. Jaskolski, B.K. Sathyanarayana, E. Baldwin, I.T. Weber, L.M. Selk, L. Clawson, J. Schneider, S.B. Kent, Conserved folding in retroviral proteases: crystal structure of a synthetic HIV1 protease, Science, 245 (1989) 616-621.

[8] J.A. Bartlett, R. DeMasi, J. Quinn, C. Moxham, F. Rousseau, Overview of the effectiveness of triple combination therapy in antiretroviral-naive HIV-1 infected adults, Aids, 15 (2001) 1369-1377.

[9] R.M. Gulick, J.W. Mellors, D. Havlir, J.J. Eron, A. Meibohm, J.H. Condra, F.T. Valentine, D. McMahon, C. Gonzalez, L. Jonas, E.A. Emini, J.A. Chodakewitz, R. Isaacs, D.D. Richman, 3-year suppression of HIV viremia with indinavir, zidovudine, and lamivudine, Annals of internal medicine, 133 (2000) 35-39.

[10] F.J. Palella, Jr., K.M. Delaney, A.C. Moorman, M.O. Loveless, J. Fuhrer, G.A. Satten, D.J. Aschman, S.D. Holmberg, Declining morbidity and mortality among patients with advanced human immunodeficiency virus infection. HIV Outpatient Study Investigators, The New England journal of medicine, 338 (1998) 853-860.

[11] R.S. Hogg, K.V. Heath, B. Yip, K.J. Craib, M.V. O'Shaughnessy, M.T. Schechter, J.S. Montaner, Improved survival among HIV-infected individuals following initiation of antiretroviral therapy, JAMA : the journal of the American Medical Association, 279 (1998) 450-454.

[12] L. Waters, M. Nelson, Why do patients fail HIV therapy?, International journal of clinical practice, 61 (2007) 983-990.

[13] J.H. Condra, W.A. Schleif, O.M. Blahy, L.J. Gabryelski, D.J. Graham, J.C. Quintero, A. Rhodes, H.L. Robbins, E. Roth, M. Shivaprakash, et al., In vivo emergence of HIV-1 variants resistant to multiple protease inhibitors, Nature, 374 (1995) 569-571.

[14] F. Clavel, A.J. Hance, HIV drug resistance, The New England journal of medicine, 350 (2004) 1023-1035.

[15] A.G. Tomasselli, R.L. Heinrikson, Targeting the HIV-protease in AIDS therapy: a current clinical perspective, Biochimica et biophysica acta, 1477 (2000) 189-214.

[16] G.S. Reddy, A. Ali, M.N. Nalam, S.G. Anjum, H. Cao, R.S. Nathans, C.A. Schiffer, T.M. Rana, Design and synthesis of HIV-1 protease inhibitors incorporating oxazolidinones as P2/P2' ligands in pseudosymmetric dipeptide isosteres, Journal of medicinal chemistry, 50 (2007) 4316-4328.

[17] A.K. Ghosh, B.D. Chapsal, I.T. Weber, H. Mitsuya, Design of HIV protease inhibitors targeting protein backbone: an effective strategy for combating drug resistance, Accounts of chemical research, 41 (2008) 78-86.

[18] A.M. Wensing, N.M. van Maarseveen, M. Nijhuis, Fifteen years of HIV Protease Inhibitors: raising the barrier to resistance, Antiviral research, 85 (2010) 59-74.

[19] A.K. Ghosh, B.D. Chapsal, A. Baldridge, M.P. Steffey, D.E. Walters, Y. Koh, M. Amano, H. Mitsuya, Design and synthesis of potent HIV-1 protease inhibitors incorporating hexahydrofuropyranol-derived high affinity $\mathrm{P}(2)$ ligands: structure-activity studies and biological evaluation, Journal of medicinal chemistry, 54 (2011) 622-634. 
[20] A.K. Ganguly, S.S. Alluri, D. Caroccia, D. Biswas, C.H. Wang, E. Kang, Y. Zhang, A.T. McPhail, S.S. Carroll, C. Burlein, V. Munshi, P. Orth, C. Strickland, Design, synthesis, and X-ray crystallographic analysis of a novel class of HIV-1 protease inhibitors, Journal of medicinal chemistry, 54 (2011) 7176-7183.

[21] X. Qiu, G.D. Zhao, L.Q. Tang, Z.P. Liu, Design and synthesis of highly potent HIV-1 protease inhibitors with novel isosorbide-derived P2 ligands, Bioorganic \& medicinal chemistry letters, 24 (2014) 2465-2468.

[22] J.V. Zivkovic, N.V. Trutic, J.B. Veselinovic, G.M. Nikolic, A.M. Veselinovic, Monte Carlo method based QSAR modeling of maleimide derivatives as glycogen synthase kinase-3beta inhibitors, Computers in biology and medicine, 64 (2015) 276-282.

[23] K. Roy, S. Kar, R.N. Das, A Primer on QSAR/QSPR Modeling Fundamental Concepts, Springer International Publishing2015.

[24] P.R. Duchowicz, N.C. Comelli, E.V. Ortiz, E.A. Castro, QSAR study for carcinogenicity in a large set of organic compounds, Current drug safety, 7 (2012) 282-288.

[25] A. Talevi, C.L. Bellera, M. Di Ianni, P.R. Duchowicz, L.E. Bruno-Blanch, E.A. Castro, An integrated drug development approach applying topological descriptors, Current computer-aided drug design, 8 (2012) 172-181.

[26] M. Randic, S.C. Basak, A new descriptor for structure-property and structure-activity correlations, Journal of chemical information and computer sciences, 41 (2001) 650-656.

[27] S. Junkes Bda, A.C. Arruda, R.A. Yunes, L.C. Porto, V.E. Heinzen, Semi-empirical topological index: a tool for QSPR/QSAR studies, Journal of molecular modeling, 11 (2005) 128-134.

[28] O. Ivanciuc, Chemical graphs, molecular matrices and topological indices in chemoinformatics and quantitative structure-activity relationships, Current computer-aided drug design, 9 (2013) 153-163.

[29] D. Weininger, SMILES, a chemical language and information system. 1. Introduction to methodology and encoding rules, Journal of chemical information and modeling, 28 (1988) 31-36.

[30] D. Weininger, A. Weininger, J.L. Weininger, SMILES. 2. Algorithm for generation of unique SMILES notation Journal of chemical information and modeling, 29 (1989) 97-101.

[31] D. Weininger, SMILES. 3. Depict. Graphical depiction of chemical structures, Journal of chemical information and modeling, 30 (1990) 237-243.

[32] A.A. Toropov, E. Benfenati, SMILES in QSPR/QSAR Modeling: results and perspectives, Current drug discovery technologies, 4 (2007) 77-116.

[33] A.A. Toropov, E. Benfenati, SMILES as an alternative to the graph in QSAR modelling of bee toxicity, Computational biology and chemistry, 31 (2007) 57-60.

[34] A.M. Veselinovic, J.B. Veselinovic, J.V. Zivkovic, G.M. Nikolic, Application of SMILES Notation Based Optimal Descriptors in Drug Discovery and Design, Current topics in medicinal chemistry, 15 (2015) 17681779.

[35] A.A. Toropov, A.P. Toropova, R.G. Diaza, E. Benfenati, G. Gini, SMILES-based optimal descriptors: QSAR modeling of estrogen receptor binding affinity by correlation balance, Structural Chemistry, 23 (2012) 529-544.

[36] A.M. Veselinovic, J.B. Milosavljevic, A.A. Toropov, G.M. Nikolic, SMILES-based QSAR models for the calcium channel-antagonistic effect of 1,4-dihydropyridines, Archiv der Pharmazie, 346 (2013) 134-139.

[37] A.M. Veselinovic, J.B. Milosavljevic, A.A. Toropov, G.M. Nikolic, SMILES-based QSAR model for arylpiperazines as high-affinity 5-HT(1A) receptor ligands using CORAL, European journal of pharmaceutical sciences : official journal of the European Federation for Pharmaceutical Sciences, 48 (2013) 532-541.

[38] J.B. Veselinovic, A.A. Toropov, A.P. Toropova, G.M. Nikolic, A.M. Veselinovic, Monte carlo methodbased QSAR modeling of penicillins binding to human serum proteins, Archiv der Pharmazie, 348 (2015) 6267.

[39] A. Worachartcheewan, P. Mandi, V. Prachayasittikul, A.P. Toropova, A.A. Toropov, C. Nantasenamat, Large-scale QSAR study of aromatase inhibitors using SMILES-based descriptors, Chemometrics and Intelligent Laboratory Systems, 138 (2014) 120-126.

[40] Q. Li, X. Ding, H. Si, H. Gao, QSAR model based on SMILES of inhibitory rate of 2, 3-diarylpropenoic acids on AKR1C3, Chemometrics and Intelligent Laboratory Systems, 139 (2014) 120-126.

[41] A.P. Toropova, A.A. Toropov, J.B. Veselinovic, F.N. Miljkovic, A.M. Veselinovic, QSAR models for HEPT derivates as NNRTI inhibitors based on Monte Carlo method, European journal of medicinal chemistry, 77 (2014) 298-305.

[42] A. Ali, G.S. Reddy, H. Cao, S.G. Anjum, M.N. Nalam, C.A. Schiffer, T.M. Rana, Discovery of HIV-1 protease inhibitors with picomolar affinities incorporating $\mathrm{N}$-aryl-oxazolidinone-5-carboxamides as novel P2 ligands, Journal of medicinal chemistry, 49 (2006) 7342-7356.

[43] A. Ali, G.S. Reddy, M.N. Nalam, S.G. Anjum, H. Cao, C.A. Schiffer, T.M. Rana, Structure-based design, synthesis, and structure-activity relationship studies of HIV-1 protease inhibitors incorporating phenyloxazolidinones, Journal of medicinal chemistry, 53 (2010) 7699-7708. 
[44] M.K. Parai, D.J. Huggins, H. Cao, M.N. Nalam, A. Ali, C.A. Schiffer, B. Tidor, T.M. Rana, Design, synthesis, and biological and structural evaluations of novel HIV-1 protease inhibitors to combat drug resistance, Journal of medicinal chemistry, 55 (2012) 6328-6341.

[45] M.A. Toropova, A.A. Toropov, I. Raska, Jr., M. Raskova, Searching therapeutic agents for treatment of Alzheimer disease using the Monte Carlo method, Computers in biology and medicine, 64 (2015) 148-154.

[46] H. Kubinyi, F.A. Hamprecht, T. Mietzner, Three-dimensional quantitative similarity-activity relationships (3D QSiAR) from SEAL similarity matrices, Journal of medicinal chemistry, 41 (1998) 2553-2564.

[47] K. Roy, I. Mitra, S. Kar, P.K. Ojha, R.N. Das, H. Kabir, Comparative studies on some metrics for external validation of QSPR models, Journal of chemical information and modeling, 52 (2012) 396-408.

[48] P.K. Ojha, I. Mitra, R.N. Das, K. Roy, Further exploring rm2 metrics for validation of QSPR models, Chemometrics and Intelligent Laboratory Systems, 107 (2011) 194-205.

[49] P. Pratim Roy, S. Paul, I. Mitra, K. Roy, On two novel parameters for validation of predictive QSAR models, Molecules, 14 (2009) 1660-1701.

[50] P.P. Roy, K. Roy, On Some Aspects of Variable Selection for Partial Least Squares Regression Models, Molecular Informatics, 27 (2007) 302-313.

[51] P.K. Ojha, R. Roy, Comparative QSARs for antimalarial endochins: importance of descriptor-thinning and noise reduction prior to feature selection, Chemometrics and Intelligent Laboratory Systems, 109 (2011) 146161.

[52] OECD, Guidance document on the validation of (quantitative) structure-activity relationships [(Q) SAR] models, ENV/JM/MONO(2007(2), Organisation for Economic Co-operation and Development, Paris, 2007.

[53] A. Tropsha, P. Gramatica, V.K. Gombar, The importance of being earnest: validation is the absolute essential for successful application and interpretation of QSPR models, 22 (2003) 69-77., QSAR \& Combinatorial Science, 22 (2003) 69-77.

[54] P.K. Ojha, I. Mitra, R.N. Das, K. Roy, Further exploring r m 2 metrics for validation of QSPR models, Chemometrics and Intelligent Laboratory Systems, 107 (2011) 194-205. 


\section{Supplementary materials}

Table S1: Observed, predicted activity and DCW, and distribution into the training (+), calibration (-), test (\#) and validation (*) sets of split 1 (Models $\mathbf{1}$ and 2)

\begin{tabular}{|c|c|c|c|c|c|c|c|}
\hline \multirow{2}{*}{$\begin{array}{l}\text { Mol. } \\
\text { No. }\end{array}$} & & \multirow{2}{*}{${ }^{1}$ Exp. } & \multicolumn{2}{|c|}{ Model 1} & \multicolumn{2}{|c|}{ Model 2} & \multirow{2}{*}{ SMILES } \\
\hline & & & ${ }^{2}$ Calc. & DCW & ${ }^{2}$ Calc. & DCW & \\
\hline $\mathrm{H} 1$ & + & 3.059 & 2.721 & 46.882 & 2.656 & 57.677 & $\begin{array}{l}\mathrm{O}=\mathrm{C}(\mathrm{O}[\mathrm{C} @ @](\mathrm{C})([\mathrm{H}]) \mathrm{CC}(=\mathrm{O}) \mathrm{OC}) \mathrm{N}[\mathrm{C} @ @]([\mathrm{H}])(\mathrm{C} / \mathrm{C} 1=\mathrm{C} / \mathrm{C}=\mathrm{C} \\
\mathrm{C}=\mathrm{C} 1)[\mathrm{C} @](\mathrm{O})([\mathrm{H}]) \mathrm{CN}(\mathrm{C}[\mathrm{C} @ @](\mathrm{C})([\mathrm{H}]) \mathrm{CC})[\mathrm{S}](=\mathrm{O})(=\mathrm{O}) \mathrm{C} 2= \\
\mathrm{CC}=\mathrm{C}(\mathrm{C}=\mathrm{C} 2) \mathrm{OC}\end{array}$ \\
\hline H5 & + & 3.371 & 3.126 & 53.864 & 3.197 & 69.425 & $\begin{array}{l}\mathrm{O}=\mathrm{C}(\mathrm{O}[\mathrm{C} @ @](\mathrm{C})([\mathrm{H}]) \mathrm{CCC}(\mathrm{C})=\mathrm{O}) \mathrm{N}[\mathrm{C} @ @]([\mathrm{H}])(\mathrm{C} / \mathrm{C} 1=\mathrm{C} / \mathrm{C}=\mathrm{C} \\
\mathrm{C}=\mathrm{C} 1)[\mathrm{C} @](\mathrm{O})([\mathrm{H}]) \mathrm{CN}(\mathrm{C}[\mathrm{C} @ @](\mathrm{C})([\mathrm{H}]) \mathrm{CC})[\mathrm{S}](=\mathrm{O})(=\mathrm{O}) \mathrm{C} 2= \\
\mathrm{CC}=\mathrm{C}(\mathrm{C}=\mathrm{C} 2) \mathrm{OC}\end{array}$ \\
\hline H6 & + & 2.697 & 3.256 & 56.104 & 3.323 & 72.156 & $\begin{array}{l}\mathrm{O}=\mathrm{C}(\mathrm{OCCNC}(=\mathrm{O}) \mathrm{OC}(\mathrm{C})(\mathrm{C}) \mathrm{C}) \mathrm{N}[\mathrm{C} @ @]([\mathrm{H}])(\mathrm{C} / \mathrm{C} 1=\mathrm{C} / \mathrm{C}=\mathrm{CC}=\mathrm{C} \\
1)[\mathrm{C} @](\mathrm{O})([\mathrm{H}]) \mathrm{CN}(\mathrm{C}[\mathrm{C} @ @](\mathrm{C})([\mathrm{H}]) \mathrm{CC})[\mathrm{S}](=\mathrm{O})(=\mathrm{O}) \mathrm{C} 2=\mathrm{CC}= \\
\mathrm{C}(\mathrm{C}=\mathrm{C} 2) \mathrm{OC}\end{array}$ \\
\hline H8 & + & 4.319 & 4.736 & 81.597 & 4.693 & 101.884 & $\begin{array}{l}\mathrm{O}=\mathrm{C}(\mathrm{OCCN} 1 \mathrm{CCNC} 1=\mathrm{O}) \mathrm{N}[\mathrm{C} @ @]([\mathrm{H}])(\mathrm{C} / \mathrm{C} 2=\mathrm{C} / \mathrm{C}=\mathrm{CC}=\mathrm{C} 2)[\mathrm{C} \\
@](\mathrm{O})([\mathrm{H}]) \mathrm{CN}(\mathrm{C}[\mathrm{C} @ @](\mathrm{C})([\mathrm{H}]) \mathrm{CC})[\mathrm{S}](=\mathrm{O})(=\mathrm{O}) \mathrm{C} 3=\mathrm{CC}=\mathrm{C}(\mathrm{C}= \\
\mathrm{C} 3) \mathrm{OC}\end{array}$ \\
\hline H12 & + & 3.229 & 2.794 & 48.131 & 2.714 & 58.928 & $\begin{array}{l}\mathrm{O}=\mathrm{C}(\mathrm{O}[\mathrm{C} @ @](\mathrm{C})([\mathrm{H}]) \mathrm{CC}(=\mathrm{O}) \mathrm{OC}) \mathrm{N}[\mathrm{C} @ @]([\mathrm{H}])(\mathrm{C} / \mathrm{C} 1=\mathrm{C} / \mathrm{C}=\mathrm{C} \\
\mathrm{C}=\mathrm{C} 1)[\mathrm{C} @](\mathrm{O})([\mathrm{H}]) \mathrm{CN}(\mathrm{C}[\mathrm{C} @ @](\mathrm{C})([\mathrm{H}]) \mathrm{CC})[\mathrm{S}](=\mathrm{O})(=\mathrm{O}) \mathrm{C} 3= \\
\mathrm{CC}=\mathrm{C} 2 \mathrm{~N}=\mathrm{C}[\mathrm{S}] \mathrm{C} 2=\mathrm{C} 3\end{array}$ \\
\hline H14 & + & 4.222 & 5.388 & 92.825 & 5.440 & 118.118 & $\begin{array}{l}\mathrm{O}=\mathrm{C}(\mathrm{OCCNC}(=\mathrm{O}) \mathrm{C}([\mathrm{Cl}])([\mathrm{Cl}])[\mathrm{H}]) \mathrm{N}[\mathrm{C} @ @]([\mathrm{H}])(\mathrm{C} / \mathrm{C} 1=\mathrm{C} / \mathrm{C}=\mathrm{C} \\
\mathrm{C}=\mathrm{C} 1)[\mathrm{C} @](\mathrm{O})([\mathrm{H}]) \mathrm{CN}(\mathrm{C}[\mathrm{C} @ @](\mathrm{C})([\mathrm{H}]) \mathrm{CC})[\mathrm{S}](=\mathrm{O})(=\mathrm{O}) \mathrm{C} 3= \\
\mathrm{CC}=\mathrm{C} 2 \mathrm{~N}=\mathrm{C}[\mathrm{S}] \mathrm{C} 2=\mathrm{C} 3\end{array}$ \\
\hline H16 & + & 3.638 & 3.329 & 57.353 & 3.381 & 73.408 & $\begin{array}{l}\mathrm{O}=\mathrm{C}(\mathrm{OCCNC}(=\mathrm{O}) \mathrm{OC}(\mathrm{C})(\mathrm{C}) \mathrm{C}) \mathrm{N}[\mathrm{C} @ @]([\mathrm{H}])(\mathrm{C} / \mathrm{C} 1=\mathrm{C} / \mathrm{C}=\mathrm{CC}=\mathrm{C} \\
1)[\mathrm{C} @](\mathrm{O})([\mathrm{H}]) \mathrm{CN}(\mathrm{C}[\mathrm{C} @ @](\mathrm{C})([\mathrm{H}]) \mathrm{CC})[\mathrm{S}](=\mathrm{O})(=\mathrm{O}) \mathrm{C} 3=\mathrm{CC}= \\
\mathrm{C} 2 \mathrm{~N}=\mathrm{C}[\mathrm{S}] \mathrm{C} 2=\mathrm{C} 3\end{array}$ \\
\hline H18 & + & 5.301 & 4.954 & 85.363 & 4.957 & 107.624 & $\begin{array}{l}\mathrm{O}=\mathrm{C}(\mathrm{OCCN} 1 \mathrm{CCNC} 1=\mathrm{O}) \mathrm{N}[\mathrm{C} @ @]([\mathrm{H}])(\mathrm{C} / \mathrm{C} 2=\mathrm{C} / \mathrm{C}=\mathrm{CC}=\mathrm{C} 2)[\mathrm{C} \\
@](\mathrm{O})([\mathrm{H}]) \mathrm{CN}(\mathrm{C}[\mathrm{C} @ @](\mathrm{C})([\mathrm{H}]) \mathrm{CC})[\mathrm{S}](=\mathrm{O})(=\mathrm{O}) \mathrm{C} 4=\mathrm{CC}=\mathrm{C} 3 \mathrm{~N} \\
=\mathrm{C}[\mathrm{S}] \mathrm{C} 3=\mathrm{C} 4\end{array}$ \\
\hline $\mathrm{H} 21$ & + & 5.523 & 4.897 & 84.375 & 4.934 & 107.127 & $\begin{array}{l}\mathrm{O}=\mathrm{C}(\mathrm{OC}[\mathrm{C} @] 1([\mathrm{H}]) \mathrm{NC}(=\mathrm{O}) \mathrm{CC} 1) \mathrm{N}[\mathrm{C} @ @]([\mathrm{H}])(\mathrm{C} / \mathrm{C} 2=\mathrm{C} / \mathrm{C}=\mathrm{CC} \\
=\mathrm{C} 2)[\mathrm{C} @](\mathrm{O})([\mathrm{H}]) \mathrm{CN}(\mathrm{C}[\mathrm{C} @ @](\mathrm{C})([\mathrm{H}]) \mathrm{CC})[\mathrm{S}](=\mathrm{O})(=\mathrm{O}) \mathrm{C} 4=\mathrm{C} \\
\mathrm{C}=\mathrm{C} 3 \mathrm{~N}=\mathrm{C}[\mathrm{S}] \mathrm{C} 3=\mathrm{C} 4\end{array}$ \\
\hline $\mathrm{H} 22$ & + & 1.763 & 2.155 & 37.133 & 2.079 & 45.158 & $\begin{array}{l}\mathrm{O}=\mathrm{C}(\mathrm{O}[\mathrm{C} @ @](\mathrm{C})([\mathrm{H}]) \mathrm{CC}(=\mathrm{O}) \mathrm{OC}) \mathrm{N}[\mathrm{C} @ @]([\mathrm{H}])(\mathrm{C} / \mathrm{C} 1=\mathrm{C} / \mathrm{C}=\mathrm{C} \\
\mathrm{C}=\mathrm{C} 1)[\mathrm{C} @](\mathrm{O})([\mathrm{H}]) \mathrm{CN}(\mathrm{CCCCC})[\mathrm{S}](=\mathrm{O})(=\mathrm{O}) \mathrm{C} 2=\mathrm{CC}=\mathrm{C}(\mathrm{C}=\mathrm{C} 2) \\
\mathrm{OC}\end{array}$ \\
\hline $\mathrm{H} 24$ & + & 2.167 & 2.560 & 44.115 & 2.620 & 56.906 & $\begin{array}{l}\mathrm{O}=\mathrm{C}(\mathrm{O}[\mathrm{C} @ @](\mathrm{C})([\mathrm{H}]) \mathrm{CCC}(\mathrm{C})=\mathrm{O}) \mathrm{N}[\mathrm{C} @ @]([\mathrm{H}])(\mathrm{C} / \mathrm{C} 1=\mathrm{C} / \mathrm{C}=\mathrm{C} \\
\mathrm{C}=\mathrm{C} 1)[\mathrm{C} @](\mathrm{O})([\mathrm{H}]) \mathrm{CN}(\mathrm{CCCCC})[\mathrm{S}](=\mathrm{O})(=\mathrm{O}) \mathrm{C} 2=\mathrm{CC}=\mathrm{C}(\mathrm{C}=\mathrm{C} 2) \\
\mathrm{OC}\end{array}$ \\
\hline $\mathrm{H} 26$ & + & 3.391 & 3.114 & 53.648 & 3.080 & 66.881 & $\begin{array}{l}\mathrm{O}=\mathrm{C}(\mathrm{OC}[\mathrm{C} @](\mathrm{C})([\mathrm{H}]) \mathrm{C}(=\mathrm{O}) \mathrm{OC}) \mathrm{N}[\mathrm{C} @ @]([\mathrm{H}])(\mathrm{C} / \mathrm{C} 1=\mathrm{C} / \mathrm{C}=\mathrm{CC} \\
=\mathrm{C} 1)[\mathrm{C} @](\mathrm{O})([\mathrm{H}]) \mathrm{CN}(\mathrm{CCCCC})[\mathrm{S}](=\mathrm{O})(=\mathrm{O}) \mathrm{C} 2=\mathrm{CC}=\mathrm{C}(\mathrm{C}=\mathrm{C} 2) \mathrm{O} \\
\mathrm{C}\end{array}$ \\
\hline $\mathrm{H} 28$ & + & 5.097 & 4.984 & 85.874 & 5.036 & 109.341 & $\begin{array}{l}\mathrm{O}=\mathrm{C}(\mathrm{OCCN} 1 \mathrm{CCOC} 1=\mathrm{O}) \mathrm{N}[\mathrm{C} @ @]([\mathrm{H}])(\mathrm{C} / \mathrm{C} 2=\mathrm{C} / \mathrm{C}=\mathrm{CC}=\mathrm{C} 2)[\mathrm{C} \\
@](\mathrm{O})([\mathrm{H}]) \mathrm{CN}(\mathrm{CCCCC})[\mathrm{S}](=\mathrm{O})(=\mathrm{O}) \mathrm{C} 3=\mathrm{CC}=\mathrm{C}(\mathrm{C}=\mathrm{C} 3) \mathrm{OC}\end{array}$ \\
\hline $\mathrm{H} 30$ & + & 3.205 & 4.113 & 70.860 & 4.093 & 88.867 & $\begin{array}{l}\mathrm{O}=\mathrm{C}(\mathrm{OC}[\mathrm{C} @] 1([\mathrm{H}]) \mathrm{NC}(=\mathrm{O}) \mathrm{CC} 1) \mathrm{N}[\mathrm{C} @ @]([\mathrm{H}])(\mathrm{C} / \mathrm{C} 2=\mathrm{C} / \mathrm{C}=\mathrm{CC} \\
=\mathrm{C} 2)[\mathrm{C} @](\mathrm{O})([\mathrm{H}]) \mathrm{CN}(\mathrm{CCCCC})[\mathrm{S}](=\mathrm{O})(=\mathrm{O}) \mathrm{C} 3=\mathrm{CC}=\mathrm{C}(\mathrm{C}=\mathrm{C} 3) \mathrm{O} \\
\mathrm{C}\end{array}$ \\
\hline
\end{tabular}




\begin{tabular}{|c|c|c|c|c|c|c|c|}
\hline H33 & + & 6.000 & 4.822 & 83.077 & 4.864 & 105.598 & $\begin{array}{l}\mathrm{O}=\mathrm{C}(\mathrm{OCCNC}(=\mathrm{O}) \mathrm{C}([\mathrm{Cl}])([\mathrm{Cl}])[\mathrm{H}]) \mathrm{N}[\mathrm{C} @ @]([\mathrm{H}])(\mathrm{C} / \mathrm{C} 1=\mathrm{C} / \mathrm{C}=\mathrm{C} \\
\mathrm{C}=\mathrm{C} 1)[\mathrm{C} @](\mathrm{O})([\mathrm{H}]) \mathrm{CN}(\mathrm{CCCCC})[\mathrm{S}](=\mathrm{O})(=\mathrm{O}) \mathrm{C} 3=\mathrm{CC}=\mathrm{C} 2 \mathrm{~N}=\mathrm{C}[ \\
\mathrm{S}] \mathrm{C} 2=\mathrm{C} 3\end{array}$ \\
\hline H35 & + & 2.889 & 2.763 & 47.604 & 2.804 & 60.888 & $\begin{array}{l}\mathrm{O}=\mathrm{C}(\mathrm{OCCNC}(=\mathrm{O}) \mathrm{OC}(\mathrm{C})(\mathrm{C}) \mathrm{C}) \mathrm{N}[\mathrm{C} @ @]([\mathrm{H}])(\mathrm{C} / \mathrm{C} 1=\mathrm{C} / \mathrm{C}=\mathrm{CC}=\mathrm{C} \\
1)[\mathrm{C} @](\mathrm{O})([\mathrm{H}]) \mathrm{CN}(\mathrm{CCCCC})[\mathrm{S}](=\mathrm{O})(=\mathrm{O}) \mathrm{C} 3=\mathrm{CC}=\mathrm{C} 2 \mathrm{~N}=\mathrm{C}[\mathrm{S}] \mathrm{C} 2 \\
=\mathrm{C} 3\end{array}$ \\
\hline H36 & + & 2.476 & 3.186 & 54.898 & 3.138 & 68.133 & $\begin{array}{l}\mathrm{O}=\mathrm{C}(\mathrm{OC}[\mathrm{C} @](\mathrm{C})([\mathrm{H}]) \mathrm{C}(=\mathrm{O}) \mathrm{OC}) \mathrm{N}[\mathrm{C} @ @]([\mathrm{H}])(\mathrm{C} / \mathrm{C} 1=\mathrm{C} / \mathrm{C}=\mathrm{CC} \\
=\mathrm{C} 1)[\mathrm{C} @](\mathrm{O})([\mathrm{H}]) \mathrm{CN}(\mathrm{CCCCC})[\mathrm{S}](=\mathrm{O})(=\mathrm{O}) \mathrm{C} 3=\mathrm{CC}=\mathrm{C} 2 \mathrm{~N}=\mathrm{C}[\mathrm{S}] \\
\mathrm{C} 2=\mathrm{C} 3\end{array}$ \\
\hline $\mathrm{H} 40$ & + & 4.569 & 4.331 & 74.626 & 4.357 & 94.607 & $\begin{array}{l}\mathrm{O}=\mathrm{C}(\mathrm{OC}[\mathrm{C} @] 1([\mathrm{H}]) \mathrm{NC}(=\mathrm{O}) \mathrm{CC} 1) \mathrm{N}[\mathrm{C} @ @]([\mathrm{H}])(\mathrm{C} / \mathrm{C} 2=\mathrm{C} / \mathrm{C}=\mathrm{CC} \\
=\mathrm{C} 2)[\mathrm{C} @](\mathrm{O})([\mathrm{H}]) \mathrm{CN}(\mathrm{CCCCC})[\mathrm{S}](=\mathrm{O})(=\mathrm{O}) \mathrm{C} 4=\mathrm{CC}=\mathrm{C} 3 \mathrm{~N}=\mathrm{C}[\mathrm{S}] \\
\mathrm{C} 3=\mathrm{C} 4\end{array}$ \\
\hline $\mathrm{H} 42$ & + & 4.081 & 4.054 & 69.851 & 3.980 & 86.416 & $\begin{array}{l}\mathrm{O}=\mathrm{C} 1 \mathrm{O}[\mathrm{C} @ @]([\mathrm{H}])(\mathrm{CN} 1 \mathrm{C}=2 \mathrm{C}=\mathrm{C} / \mathrm{C}=\mathrm{C}(/[\mathrm{F}]) \mathrm{C}=2) \mathrm{C}(=\mathrm{O}) \mathrm{N}[\mathrm{C} @ \\
@]([\mathrm{H}])(\mathrm{C} / \mathrm{C} 3=\mathrm{C} / \mathrm{C}=\mathrm{CC}=\mathrm{C} 3)[\mathrm{C} @ @](\mathrm{O})([\mathrm{H}]) \mathrm{CN}(\mathrm{CC}(\mathrm{C})(\mathrm{C})[\mathrm{H}])[ \\
\mathrm{S}](=\mathrm{O})(=\mathrm{O}) \mathrm{C} 4=\mathrm{CC}=\mathrm{C}(\mathrm{C}=\mathrm{C} 4) \mathrm{OC}\end{array}$ \\
\hline H45 & + & 6.097 & 4.721 & 81.346 & 4.623 & 100.384 & $\begin{array}{l}\mathrm{O}=\mathrm{C} 1 \mathrm{O}[\mathrm{C} @ @]([\mathrm{H}])(\mathrm{CN} 1 \mathrm{C}=2 \mathrm{C}=\mathrm{CC}=\mathrm{C}(\mathrm{C}=2) \mathrm{C}(\mathrm{C})=\mathrm{O}) \mathrm{C}(=\mathrm{O}) \mathrm{N}[ \\
\mathrm{C} @ @]([\mathrm{H}])(\mathrm{C} / \mathrm{C} 3=\mathrm{C} / \mathrm{C}=\mathrm{CC}=\mathrm{C} 3)[\mathrm{C} @ @](\mathrm{O})([\mathrm{H}]) \mathrm{CN}(\mathrm{CC}(\mathrm{C})(\mathrm{C})[ \\
\mathrm{H}])[\mathrm{S}](=\mathrm{O})(=\mathrm{O}) \mathrm{C} 4=\mathrm{CC}=\mathrm{C}(\mathrm{C}=\mathrm{C} 4) \mathrm{OC}\end{array}$ \\
\hline $\mathrm{H} 48$ & + & 3.276 & 3.432 & 59.126 & 3.414 & 74.135 & $\begin{array}{l}\mathrm{O}=\mathrm{C} 1 \mathrm{O}[\mathrm{C} @ @]([\mathrm{H}])(\mathrm{CN} 1 \mathrm{C}=2 \mathrm{C}=\mathrm{CC}=\mathrm{CC}=2) \mathrm{C}(=\mathrm{O}) \mathrm{N}[\mathrm{C} @ @]([\mathrm{H}] \\
)(\mathrm{C} / \mathrm{C} 3=\mathrm{C} / \mathrm{C}=\mathrm{CC}=\mathrm{C} 3)[\mathrm{C} @ @](\mathrm{O})([\mathrm{H}]) \mathrm{CN}(\mathrm{CC}(\mathrm{C})(\mathrm{C})[\mathrm{H}])[\mathrm{S}](=\mathrm{O})( \\
=\mathrm{O}) \mathrm{C} 4=\mathrm{C} / \mathrm{C}=\mathrm{C}(/ \mathrm{N}) \mathrm{C}=\mathrm{C} 4\end{array}$ \\
\hline H49 & + & 3.770 & 3.605 & 62.110 & 3.624 & 78.683 & $\begin{array}{l}\mathrm{O}=\mathrm{C} 1 \mathrm{O}[\mathrm{C} @ @]([\mathrm{H}])(\mathrm{CN} 1 \mathrm{C}=2 \mathrm{C}=\mathrm{C} / \mathrm{C}=\mathrm{C}(/[\mathrm{F}]) \mathrm{C}=2) \mathrm{C}(=\mathrm{O}) \mathrm{N}[\mathrm{C} @ \\
@]([\mathrm{H}])(\mathrm{C} / \mathrm{C} 3=\mathrm{C} / \mathrm{C}=\mathrm{CC}=\mathrm{C} 3)[\mathrm{C} @ @](\mathrm{O})([\mathrm{H}]) \mathrm{CN}(\mathrm{CC}(\mathrm{C})(\mathrm{C})[\mathrm{H}])[ \\
\mathrm{S}](=\mathrm{O})(=\mathrm{O}) \mathrm{C} 4=\mathrm{C} / \mathrm{C}=\mathrm{C}(/ \mathrm{N}) \mathrm{C}=\mathrm{C} 4\end{array}$ \\
\hline H51 & + & 4.377 & 3.442 & 59.311 & 3.704 & 80.436 & $\begin{array}{l}\mathrm{O}=\mathrm{C} 1 \mathrm{O}[\mathrm{C} @ @]([\mathrm{H}])(\mathrm{CN} 1 \mathrm{C}=2 \mathrm{C}=\mathrm{CC}=\mathrm{C}(\mathrm{C}=2) \mathrm{C}([\mathrm{F}])([\mathrm{F}])[\mathrm{F}]) \mathrm{C}(= \\
\mathrm{O}) \mathrm{N}[\mathrm{C} @ @]([\mathrm{H}])(\mathrm{C} / \mathrm{C} 3=\mathrm{C} / \mathrm{C}=\mathrm{CC}=\mathrm{C} 3)[\mathrm{C} @ @](\mathrm{O})([\mathrm{H}]) \mathrm{CN}(\mathrm{CC}(\mathrm{C} \\
)(\mathrm{C})[\mathrm{H}])[\mathrm{S}](=\mathrm{O})(=\mathrm{O}) \mathrm{C} 4=\mathrm{C} / \mathrm{C}=\mathrm{C}(/ \mathrm{N}) \mathrm{C}=\mathrm{C} 4\end{array}$ \\
\hline H53 & + & 3.735 & 3.649 & 62.862 & 3.553 & 77.142 & $\begin{array}{l}\mathrm{O}=\mathrm{C} 1 \mathrm{O}[\mathrm{C} @ @]([\mathrm{H}])(\mathrm{CN} 1 \mathrm{C}=2 \mathrm{C}=\mathrm{CC}(=\mathrm{CC}=2) \mathrm{C}(=\mathrm{O}) \mathrm{C}) \mathrm{C}(=\mathrm{O}) \mathrm{N}[ \\
\mathrm{C} @ @]([\mathrm{H}])(\mathrm{C} / \mathrm{C} 3=\mathrm{C} / \mathrm{C}=\mathrm{CC}=\mathrm{C} 3)[\mathrm{C} @ @](\mathrm{O})([\mathrm{H}]) \mathrm{CN}(\mathrm{CC}(\mathrm{C})(\mathrm{C})[ \\
\mathrm{H}])[\mathrm{S}](=\mathrm{O})(=\mathrm{O}) \mathrm{C} 4=\mathrm{C} / \mathrm{C}=\mathrm{C}(/ \mathrm{N}) \mathrm{C}=\mathrm{C} 4\end{array}$ \\
\hline H54 & + & 3.971 & 3.924 & 67.605 & 3.899 & 84.662 & $\begin{array}{l}\mathrm{O}=\mathrm{C} 1 \mathrm{O}[\mathrm{C} @ @]([\mathrm{H}])(\mathrm{CN} 1 \mathrm{C}=2 \mathrm{C}=\mathrm{C} / \mathrm{C}=\mathrm{C}(/[\mathrm{F}]) \mathrm{C}=2) \mathrm{C}(=\mathrm{O}) \mathrm{N}[\mathrm{C} @ \\
@]([\mathrm{H}])(\mathrm{C} / \mathrm{C} 3=\mathrm{C} / \mathrm{C}=\mathrm{CC}=\mathrm{C} 3)[\mathrm{C} @ @](\mathrm{O})([\mathrm{H}]) \mathrm{CN}(\mathrm{CC}(\mathrm{C})(\mathrm{C})[\mathrm{H}])[ \\
\mathrm{S}](=\mathrm{O})(=\mathrm{O}) \mathrm{C} 5=\mathrm{CC}=\mathrm{C} 4 \mathrm{OCOC} 4=\mathrm{C} 5\end{array}$ \\
\hline H57 & + & 5.222 & 4.591 & 79.100 & 4.543 & 98.630 & $\begin{array}{l}\mathrm{O}=\mathrm{C} 1 \mathrm{O}[\mathrm{C} @ @]([\mathrm{H}])(\mathrm{CN} 1 \mathrm{C}=2 \mathrm{C}=\mathrm{CC}=\mathrm{C}(\mathrm{C}=2) \mathrm{C}(=\mathrm{O}) \mathrm{C}) \mathrm{C}(=\mathrm{O}) \mathrm{N}[ \\
\mathrm{C} @ @]([\mathrm{H}])(\mathrm{C} / \mathrm{C} 3=\mathrm{C} / \mathrm{C}=\mathrm{CC}=\mathrm{C} 3)[\mathrm{C} @ @](\mathrm{O})([\mathrm{H}]) \mathrm{CN}(\mathrm{CC}(\mathrm{C})(\mathrm{C})[ \\
\mathrm{H}])[\mathrm{S}](=\mathrm{O})(=\mathrm{O}) \mathrm{C} 5=\mathrm{CC}=\mathrm{C} 4 \mathrm{OCOC} 4=\mathrm{C} 5\end{array}$ \\
\hline H59 & + & 4.155 & 4.040 & 69.606 & 4.015 & 87.187 & $\begin{array}{l}\mathrm{O}=\mathrm{C} 1 \mathrm{O}[\mathrm{C} @ @]([\mathrm{H}])(\mathrm{CN} 1 \mathrm{C}=2 \mathrm{C}=\mathrm{C} / \mathrm{C}=\mathrm{C}(/[\mathrm{F}]) \mathrm{C}=2) \mathrm{C}(=\mathrm{O}) \mathrm{N}[\mathrm{C} @ \\
@]([\mathrm{H}])(\mathrm{C} / \mathrm{C} 3=\mathrm{C} / \mathrm{C}=\mathrm{CC}=\mathrm{C} 3)[\mathrm{C} @ @](\mathrm{O})([\mathrm{H}]) \mathrm{CN}(\mathrm{CC}(\mathrm{C})(\mathrm{C})[\mathrm{H}])[ \\
\mathrm{S}](=\mathrm{O})(=\mathrm{O}) \mathrm{C} 4=\mathrm{C} / \mathrm{C}=\mathrm{C}(/ \mathrm{OC}) \mathrm{C}(\mathrm{C})=\mathrm{C} 4\end{array}$ \\
\hline H62 & + & 3.876 & 4.707 & 81.102 & 4.659 & 101.155 & $\begin{array}{l}\mathrm{O}=\mathrm{C} 1 \mathrm{O}[\mathrm{C} @ @]([\mathrm{H}])(\mathrm{CN} 1 \mathrm{C}=2 \mathrm{C}=\mathrm{CC}=\mathrm{C}(\mathrm{C}=2) \mathrm{C}(=\mathrm{O}) \mathrm{C}) \mathrm{C}(=\mathrm{O}) \mathrm{N}[ \\
\mathrm{C} @ @]([\mathrm{H}])(\mathrm{C} / \mathrm{C} 3=\mathrm{C} / \mathrm{C}=\mathrm{CC}=\mathrm{C} 3)[\mathrm{C} @ @](\mathrm{O})([\mathrm{H}]) \mathrm{CN}(\mathrm{CC}(\mathrm{C})(\mathrm{C})[ \\
\mathrm{H}])[\mathrm{S}](=\mathrm{O})(=\mathrm{O}) \mathrm{C} 4=\mathrm{C} / \mathrm{C}=\mathrm{C}(/ \mathrm{OC}) \mathrm{C}(\mathrm{C})=\mathrm{C} 4\end{array}$ \\
\hline H63 & + & 4.097 & 4.084 & 70.358 & 3.944 & 85.645 & $\begin{array}{l}\mathrm{O}=\mathrm{C} 1 \mathrm{O}[\mathrm{C} @ @]([\mathrm{H}])(\mathrm{CN} 1 \mathrm{C}=2 \mathrm{C}=\mathrm{CC}(=\mathrm{CC}=2) \mathrm{C}(=\mathrm{O}) \mathrm{C}) \mathrm{C}(=\mathrm{O}) \mathrm{N}[ \\
\mathrm{C} @ @]([\mathrm{H}])(\mathrm{C} / \mathrm{C} 3=\mathrm{C} / \mathrm{C}=\mathrm{CC}=\mathrm{C} 3)[\mathrm{C} @ @](\mathrm{O})([\mathrm{H}]) \mathrm{CN}(\mathrm{CC}(\mathrm{C})(\mathrm{C})[ \\
\mathrm{H}])[\mathrm{S}](=\mathrm{O})(=\mathrm{O}) \mathrm{C} 4=\mathrm{C} / \mathrm{C}=\mathrm{C}(/ \mathrm{OC}) \mathrm{C}(\mathrm{C})=\mathrm{C} 4\end{array}$ \\
\hline H65 & + & 2.699 & 3.383 & 58.290 & 3.508 & 76.171 & $\begin{array}{l}\mathrm{O}=\mathrm{C} 1 \mathrm{O}[\mathrm{C} @ @]([\mathrm{H}])(\mathrm{CN} 1 \mathrm{C}=2 \mathrm{C}=\mathrm{CC}=\mathrm{C}(\mathrm{C}=2) \mathrm{C}(=\mathrm{O}) \mathrm{C}) \mathrm{C}(=\mathrm{O}) \mathrm{N}[ \\
\mathrm{C} @ @]([\mathrm{H}])(\mathrm{C} / \mathrm{C} 3=\mathrm{C} / \mathrm{C}=\mathrm{CC}=\mathrm{C} 3)[\mathrm{C} @ @](\mathrm{O})([\mathrm{H}]) \mathrm{CN}(\mathrm{CC}(\mathrm{C})(\mathrm{C})[ \\
\mathrm{H}])[\mathrm{S}](=\mathrm{O})(=\mathrm{O}) \mathrm{C} 4=\mathrm{CC}=\mathrm{C}(\mathrm{C}=\mathrm{C} 4) \mathrm{OC}([\mathrm{F}])([\mathrm{F}])[\mathrm{F}]\end{array}$ \\
\hline H66 & + & 2.420 & 2.543 & 43.810 & 2.655 & 57.654 & $\mathrm{O}=\mathrm{C} 1 \mathrm{O}[\mathrm{C} @ @]([\mathrm{H}])(\mathrm{CN} 1 \mathrm{C}=2 \mathrm{C}=\mathrm{CC}=\mathrm{CC}=2) \mathrm{C}(=\mathrm{O}) \mathrm{N}[\mathrm{C} @ @]([\mathrm{H}]$ \\
\hline
\end{tabular}




\begin{tabular}{|c|c|c|c|c|c|c|c|}
\hline & & & & & & & $\begin{array}{l})(\mathrm{C} / \mathrm{C} 3=\mathrm{C} / \mathrm{C}=\mathrm{CC}=\mathrm{C} 3)[\mathrm{C} @ @](\mathrm{O})([\mathrm{H}]) \mathrm{CN}(\mathrm{CC}(\mathrm{C})(\mathrm{C})[\mathrm{H}])[\mathrm{S}](=\mathrm{O})( \\
=\mathrm{O}) \mathrm{C} 4=\mathrm{CC}=\mathrm{C}(\mathrm{C}=\mathrm{C} 4) \mathrm{OC}([\mathrm{F}])([\mathrm{F}])[\mathrm{F}]\end{array}$ \\
\hline H67 & + & 3.076 & 2.760 & 47.546 & 2.793 & 60.662 & $\begin{array}{l}\mathrm{O}=\mathrm{C} 1 \mathrm{O}[\mathrm{C} @ @]([\mathrm{H}])(\mathrm{CN} 1 \mathrm{C}=2 \mathrm{C}=\mathrm{CC}(=\mathrm{CC}=2) \mathrm{C}(=\mathrm{O}) \mathrm{C}) \mathrm{C}(=\mathrm{O}) \mathrm{N}[ \\
\mathrm{C} @ @]([\mathrm{H}])(\mathrm{C} / \mathrm{C} 3=\mathrm{C} / \mathrm{C}=\mathrm{CC}=\mathrm{C} 3)[\mathrm{C} @ @](\mathrm{O})([\mathrm{H}]) \mathrm{CN}(\mathrm{CC}(\mathrm{C})(\mathrm{C})[ \\
\mathrm{H}])[\mathrm{S}](=\mathrm{O})(=\mathrm{O}) \mathrm{C} 4=\mathrm{CC}=\mathrm{C}(\mathrm{C}=\mathrm{C} 4) \mathrm{OC}([\mathrm{F}])([\mathrm{F}])[\mathrm{F}]\end{array}$ \\
\hline H69 & + & 3.237 & 3.705 & 63.841 & 3.634 & 78.905 & $\begin{array}{l}\mathrm{O}=\mathrm{C} 1 \mathrm{O}[\mathrm{C} @ @]([\mathrm{H}])(\mathrm{CN} 1 \mathrm{C}=2 \mathrm{C}=\mathrm{CC}([\mathrm{F}])=\mathrm{C}([\mathrm{F}]) \mathrm{C}=2) \mathrm{C}(=\mathrm{O}) \mathrm{N}[\mathrm{C} \\
@ @]([\mathrm{H}])(\mathrm{C} / \mathrm{C} 3=\mathrm{C} / \mathrm{C}=\mathrm{CC}=\mathrm{C} 3)[\mathrm{C} @ @](\mathrm{O})([\mathrm{H}]) \mathrm{CN}(\mathrm{C}[\mathrm{C} @ @] 4([ \\
\mathrm{H}]) \mathrm{CC} 4)[\mathrm{S}](=\mathrm{O})(=\mathrm{O}) \mathrm{C} 5=\mathrm{CC}=\mathrm{C}(\mathrm{C}=\mathrm{C} 5) \mathrm{OC}\end{array}$ \\
\hline H71 & + & 0.622 & 0.807 & 13.895 & 0.820 & 17.824 & $\begin{array}{l}\mathrm{O}=\mathrm{C} 1 \mathrm{O}[\mathrm{C} @ @]([\mathrm{H}])(\mathrm{CN} 1 \mathrm{C}=2 \mathrm{C}=\mathrm{CC}=\mathrm{CC}=2) \mathrm{C}(=\mathrm{O}) \mathrm{N}[\mathrm{C} @ @]([\mathrm{H}] \\
)(\mathrm{C} / \mathrm{C} 3=\mathrm{C} / \mathrm{C}=\mathrm{CC}=\mathrm{C} 3)[\mathrm{C} @ @](\mathrm{O})([\mathrm{H}]) \mathrm{CN}(\mathrm{C} / \mathrm{C} 4=\mathrm{C} / \mathrm{C}=\mathrm{C}[\mathrm{S}] 4)[\mathrm{S}]( \\
=\mathrm{O})(=\mathrm{O}) \mathrm{C} 5=\mathrm{CC}=\mathrm{CC}(=\mathrm{C} 5) \mathrm{OC}\end{array}$ \\
\hline $\mathrm{H} 72$ & + & 0.724 & 0.980 & 16.879 & 1.030 & 22.372 & $\begin{array}{l}\mathrm{O}=\mathrm{C} 1 \mathrm{O}[\mathrm{C} @ @]([\mathrm{H}])(\mathrm{CN} 1 \mathrm{C}=2 \mathrm{C}=\mathrm{C} / \mathrm{C}=\mathrm{C}(/[\mathrm{F}]) \mathrm{C}=2) \mathrm{C}(=\mathrm{O}) \mathrm{N}[\mathrm{C} @ \\
@]([\mathrm{H}])(\mathrm{C} / \mathrm{C} 3=\mathrm{C} / \mathrm{C}=\mathrm{CC}=\mathrm{C} 3)[\mathrm{C} @ @](\mathrm{O})([\mathrm{H}]) \mathrm{CN}(\mathrm{C} / \mathrm{C} 4=\mathrm{C} / \mathrm{C}=\mathrm{C}[ \\
\mathrm{S}] 4)[\mathrm{S}](=\mathrm{O})(=\mathrm{O}) \mathrm{C} 5=\mathrm{CC}=\mathrm{CC}(=\mathrm{C} 5) \mathrm{OC}\end{array}$ \\
\hline H73 & + & 1.530 & 1.023 & 17.631 & 0.959 & 20.831 & $\begin{array}{l}\mathrm{O}=\mathrm{C} 1 \mathrm{O}[\mathrm{C} @ @]([\mathrm{H}])(\mathrm{CN} 1 \mathrm{C}=2 \mathrm{C}=\mathrm{CC}(=\mathrm{CC}=2) \mathrm{C}(=\mathrm{O}) \mathrm{C}) \mathrm{C}(=\mathrm{O}) \mathrm{N}[ \\
\mathrm{C} @ @]([\mathrm{H}])(\mathrm{C} / \mathrm{C} 3=\mathrm{C} / \mathrm{C}=\mathrm{CC}=\mathrm{C} 3)[\mathrm{C} @ @](\mathrm{O})([\mathrm{H}]) \mathrm{CN}(\mathrm{C} / \mathrm{C} 4=\mathrm{C} / \mathrm{C} \\
=\mathrm{C}[\mathrm{S}] 4)[\mathrm{S}](=\mathrm{O})(=\mathrm{O}) \mathrm{C} 5=\mathrm{CC}=\mathrm{CC}(=\mathrm{C} 5) \mathrm{OC}\end{array}$ \\
\hline H77 & + & 1.377 & 1.009 & 17.391 & 0.923 & 20.067 & $\begin{array}{l}\mathrm{O}=\mathrm{C} 1 \mathrm{O}[\mathrm{C} @ @]([\mathrm{H}])(\mathrm{CN} 1 \mathrm{C}=2 \mathrm{C}=\mathrm{CC}=\mathrm{CC}=2) \mathrm{C}(=\mathrm{O}) \mathrm{N}[\mathrm{C} @ @]([\mathrm{H}] \\
)(\mathrm{C} / \mathrm{C} 3=\mathrm{C} / \mathrm{C}=\mathrm{CC}=\mathrm{C} 3)[\mathrm{C} @ @](\mathrm{O})([\mathrm{H}]) \mathrm{CN}(\mathrm{C}[\mathrm{C} @ @] 4([\mathrm{H}]) \mathrm{CCCO} \\
4)[\mathrm{S}](=\mathrm{O})(=\mathrm{O}) \mathrm{C} 5=\mathrm{CC}=\mathrm{CC}(=\mathrm{C} 5) \mathrm{OC}\end{array}$ \\
\hline H78 & + & 0.824 & 1.183 & 20.374 & 1.133 & 24.616 & $\begin{array}{l}\mathrm{O}=\mathrm{C} 1 \mathrm{O}[\mathrm{C} @ @]([\mathrm{H}])(\mathrm{CN} 1 \mathrm{C}=2 \mathrm{C}=\mathrm{C} / \mathrm{C}=\mathrm{C}(/[\mathrm{F}]) \mathrm{C}=2) \mathrm{C}(=\mathrm{O}) \mathrm{N}[\mathrm{C} @ \\
@]([\mathrm{H}])(\mathrm{C} / \mathrm{C} 3=\mathrm{C} / \mathrm{C}=\mathrm{CC}=\mathrm{C} 3)[\mathrm{C} @ @](\mathrm{O})([\mathrm{H}]) \mathrm{CN}(\mathrm{C}[\mathrm{C} @ @] 4([\mathrm{H}] \\
) \mathrm{CCCO} 4)[\mathrm{S}](=\mathrm{O})(=\mathrm{O}) \mathrm{C} 5=\mathrm{CC}=\mathrm{CC}(=\mathrm{C} 5) \mathrm{OC}\end{array}$ \\
\hline H79 & + & 3.686 & 3.751 & 64.621 & 3.690 & 80.114 & $\begin{array}{l}\mathrm{O}=\mathrm{C} 1 \mathrm{O}[\mathrm{C} @ @]([\mathrm{H}])(\mathrm{CN} 1 \mathrm{C}=2 \mathrm{C}=\mathrm{CC}=\mathrm{CC}=2) \mathrm{C}(=\mathrm{O}) \mathrm{N}[\mathrm{C} @ @]([\mathrm{H}] \\
)(\mathrm{C} / \mathrm{C} 3=\mathrm{C} / \mathrm{C}=\mathrm{CC}=\mathrm{C} 3)[\mathrm{C} @ @](\mathrm{O})([\mathrm{H}]) \mathrm{CN}(\mathrm{CC}(\mathrm{C})(\mathrm{C})[\mathrm{H}])[\mathrm{S}](=\mathrm{O})( \\
=\mathrm{O}) \mathrm{C} 5=\mathrm{CC}=\mathrm{C} 4 \mathrm{OCOC} 4=\mathrm{C} 5\end{array}$ \\
\hline H81 & + & 3.599 & 3.300 & 56.864 & 3.264 & 70.868 & $\begin{array}{l}\mathrm{O}=\mathrm{C} 1 \mathrm{O}[\mathrm{C} @ @]([\mathrm{H}])(\mathrm{CN} 1 \mathrm{C}=2 \mathrm{C}=\mathrm{CC}=\mathrm{CC}=2) \mathrm{C}(=\mathrm{O}) \mathrm{N}[\mathrm{C} @ @]([\mathrm{H}] \\
)(\mathrm{C} / \mathrm{C} 3=\mathrm{C} / \mathrm{C}=\mathrm{CC}=\mathrm{C} 3)[\mathrm{C} @ @](\mathrm{O})([\mathrm{H}]) \mathrm{CN}(\mathrm{CC}(\mathrm{C})(\mathrm{C})[\mathrm{H}])[\mathrm{S}](=\mathrm{O})( \\
=\mathrm{O}) \mathrm{C} 4=\mathrm{CC}=\mathrm{C}(\mathrm{C}=\mathrm{C} 4) \mathrm{CO}\end{array}$ \\
\hline H83 & + & 2.248 & 2.516 & 43.353 & 2.503 & 54.356 & $\begin{array}{l}\mathrm{O}=\mathrm{C} 1 \mathrm{O}[\mathrm{C} @ @]([\mathrm{H}])(\mathrm{CN} 1 \mathrm{C}=2 \mathrm{C}=\mathrm{CC}=\mathrm{CC}=2 \mathrm{O}) \mathrm{C}(=\mathrm{O}) \mathrm{N}[\mathrm{C} @ @]([ \\
\mathrm{H}])(\mathrm{C} / \mathrm{C} 3=\mathrm{C} / \mathrm{C}=\mathrm{CC}=\mathrm{C} 3)[\mathrm{C} @ @](\mathrm{O})([\mathrm{H}]) \mathrm{CN}(\mathrm{CC}(\mathrm{C})(\mathrm{C})[\mathrm{H}])[\mathrm{S}](= \\
\mathrm{O})(=\mathrm{O}) \mathrm{C} 4=\mathrm{CC}=\mathrm{C}(\mathrm{C}=\mathrm{C} 4) \mathrm{OC}\end{array}$ \\
\hline H85 & + & 3.866 & 3.675 & 63.325 & 3.692 & 80.160 & $\begin{array}{l}\mathrm{O}=\mathrm{C} 1 \mathrm{O}[\mathrm{C} @ @]([\mathrm{H}])(\mathrm{CN} 1 \mathrm{C}=2 \mathrm{C}=\mathrm{CC}=\mathrm{CC}=2 \mathrm{C}([\mathrm{F}])([\mathrm{F}])[\mathrm{F}]) \mathrm{C}(=\mathrm{O} \\
) \mathrm{N}[\mathrm{C} @ @]([\mathrm{H}])(\mathrm{C} / \mathrm{C} 3=\mathrm{C} / \mathrm{C}=\mathrm{CC}=\mathrm{C} 3)[\mathrm{C} @ @](\mathrm{O})([\mathrm{H}]) \mathrm{CN}(\mathrm{CC}(\mathrm{C})( \\
\mathrm{C})[\mathrm{H}])[\mathrm{S}](=\mathrm{O})(=\mathrm{O}) \mathrm{C} 4=\mathrm{C} / \mathrm{C}=\mathrm{C}(/ \mathrm{N}) \mathrm{C}=\mathrm{C} 4\end{array}$ \\
\hline H87 & + & 3.848 & 3.994 & 68.820 & 3.967 & 86.139 & $\begin{array}{l}\mathrm{O}=\mathrm{C} 1 \mathrm{O}[\mathrm{C} @ @]([\mathrm{H}])(\mathrm{CN} 1 \mathrm{C}=2 \mathrm{C}=\mathrm{CC}=\mathrm{CC}=2 \mathrm{C}([\mathrm{F}])([\mathrm{F}])[\mathrm{F}]) \mathrm{C}(=\mathrm{O} \\
) \mathrm{N}[\mathrm{C} @ @]([\mathrm{H}])(\mathrm{C} / \mathrm{C} 3=\mathrm{C} / \mathrm{C}=\mathrm{CC}=\mathrm{C} 3)[\mathrm{C} @ @](\mathrm{O})([\mathrm{H}]) \mathrm{CN}(\mathrm{CC}(\mathrm{C})( \\
\mathrm{C})[\mathrm{H}])[\mathrm{S}](=\mathrm{O})(=\mathrm{O}) \mathrm{C} 5=\mathrm{CC}=\mathrm{C} 4 \mathrm{OCOC} 4=\mathrm{C} 5\end{array}$ \\
\hline H89 & + & 3.629 & 3.544 & 61.063 & 3.541 & 76.893 & $\begin{array}{l}\mathrm{O}=\mathrm{C} 1 \mathrm{O}[\mathrm{C} @ @]([\mathrm{H}])(\mathrm{CN} 1 \mathrm{C}=2 \mathrm{C}=\mathrm{CC}=\mathrm{CC}=2 \mathrm{C}([\mathrm{F}])([\mathrm{F}])[\mathrm{F}]) \mathrm{C}(=\mathrm{O} \\
) \mathrm{N}[\mathrm{C} @ @]([\mathrm{H}])(\mathrm{C} / \mathrm{C} 3=\mathrm{C} / \mathrm{C}=\mathrm{CC}=\mathrm{C} 3)[\mathrm{C} @ @](\mathrm{O})([\mathrm{H}]) \mathrm{CN}(\mathrm{CC}(\mathrm{C})( \\
\mathrm{C})[\mathrm{H}])[\mathrm{S}](=\mathrm{O})(=\mathrm{O}) \mathrm{C} 4=\mathrm{CC}=\mathrm{C}(\mathrm{C}=\mathrm{C} 4) \mathrm{CO}\end{array}$ \\
\hline H91 & + & 4.201 & 3.791 & 65.320 & 3.726 & 80.903 & $\begin{array}{l}\mathrm{O}=\mathrm{C} 1 \mathrm{O}[\mathrm{C} @ @]([\mathrm{H}])(\mathrm{CN} 1 \mathrm{C}=2 \mathrm{C}=\mathrm{CC}([\mathrm{F}])=\mathrm{CC}=2[\mathrm{~F}]) \mathrm{C}(=\mathrm{O}) \mathrm{N}[\mathrm{C} \\
@ @]([\mathrm{H}])(\mathrm{C} / \mathrm{C} 3=\mathrm{C} / \mathrm{C}=\mathrm{CC}=\mathrm{C} 3)[\mathrm{C} @ @](\mathrm{O})([\mathrm{H}]) \mathrm{CN}(\mathrm{CC}(\mathrm{C})(\mathrm{C})[\mathrm{H} \\
])[\mathrm{S}](=\mathrm{O})(=\mathrm{O}) \mathrm{C} 4=\mathrm{CC}=\mathrm{C}(\mathrm{C}=\mathrm{C} 4) \mathrm{OC}\end{array}$ \\
\hline H92 & + & 3.460 & 3.661 & 63.074 & 3.645 & 79.150 & $\begin{array}{l}\mathrm{O}=\mathrm{C} 1 \mathrm{O}[\mathrm{C} @ @]([\mathrm{H}])(\mathrm{CN} 1 \mathrm{C}=2 \mathrm{C}=\mathrm{CC}([\mathrm{F}])=\mathrm{CC}=2[\mathrm{~F}]) \mathrm{C}(=\mathrm{O}) \mathrm{N}[\mathrm{C} \\
@ @]([\mathrm{H}])(\mathrm{C} / \mathrm{C} 3=\mathrm{C} / \mathrm{C}=\mathrm{CC}=\mathrm{C} 3)[\mathrm{C} @ @](\mathrm{O})([\mathrm{H}]) \mathrm{CN}(\mathrm{CC}(\mathrm{C})(\mathrm{C})[\mathrm{H} \\
])[\mathrm{S}](=\mathrm{O})(=\mathrm{O}) \mathrm{C} 5=\mathrm{CC}=\mathrm{C} 4 \mathrm{OCOC} 4=\mathrm{C} 5\end{array}$ \\
\hline H93 & + & 3.824 & 4.037 & 69.562 & 4.061 & 88.168 & $\begin{array}{l}\mathrm{O}=\mathrm{C} 1 \mathrm{O}[\mathrm{C} @ @]([\mathrm{H}])(\mathrm{CN} 1 \mathrm{C}=2 \mathrm{C}=\mathrm{CC}([\mathrm{F}])=\mathrm{CC}=2[\mathrm{~F}]) \mathrm{C}(=\mathrm{O}) \mathrm{N}[\mathrm{C} \\
@ @]([\mathrm{H}])(\mathrm{C} / \mathrm{C} 3=\mathrm{C} / \mathrm{C}=\mathrm{CC}=\mathrm{C} 3)[\mathrm{C} @ @](\mathrm{O})([\mathrm{H}]) \mathrm{CN}(\mathrm{CC}(\mathrm{C})(\mathrm{C})[\mathrm{H}\end{array}$ \\
\hline
\end{tabular}




\begin{tabular}{|c|c|c|c|c|c|c|c|}
\hline & & & & & & & ]$)[\mathrm{S}](=\mathrm{O})(=\mathrm{O}) \mathrm{C} 5=\mathrm{CC}=\mathrm{C} 4 \mathrm{~N}=\mathrm{C}[\mathrm{S}] \mathrm{C} 4=\mathrm{C} 5$ \\
\hline H95 & + & 3.349 & 3.329 & 57.357 & 3.542 & 76.916 & $\begin{array}{l}\mathrm{O}=\mathrm{C} 1 \mathrm{O}[\mathrm{C} @ @]([\mathrm{H}])(\mathrm{CN} 1 \mathrm{C}=2 \mathrm{C}=\mathrm{CC}([\mathrm{F}])=\mathrm{CC}=2) \mathrm{C}(=\mathrm{O}) \mathrm{N}[\mathrm{C} @ @ \\
]([\mathrm{H}])(\mathrm{C} / \mathrm{C} 3=\mathrm{C} / \mathrm{C}=\mathrm{CC}=\mathrm{C} 3)[\mathrm{C} @ @](\mathrm{O})([\mathrm{H}]) \mathrm{CN}(\mathrm{CC}(\mathrm{C})(\mathrm{C})[\mathrm{H}])[\mathrm{S}] \\
(=\mathrm{O})(=\mathrm{O}) \mathrm{C} 4=\mathrm{C} / \mathrm{C}=\mathrm{C}(/ \mathrm{N}) \mathrm{C}=\mathrm{C} 4\end{array}$ \\
\hline H97 & + & 3.777 & 3.648 & 62.851 & 3.818 & 82.895 & $\begin{array}{l}\mathrm{O}=\mathrm{C} 1 \mathrm{O}[\mathrm{C} @ @]([\mathrm{H}])(\mathrm{CN} 1 \mathrm{C}=2 \mathrm{C}=\mathrm{CC}([\mathrm{F}])=\mathrm{CC}=2) \mathrm{C}(=\mathrm{O}) \mathrm{N}[\mathrm{C} @ @ \\
]([\mathrm{H}])(\mathrm{C} / \mathrm{C} 3=\mathrm{C} / \mathrm{C}=\mathrm{CC}=\mathrm{C} 3)[\mathrm{C} @ @](\mathrm{O})([\mathrm{H}]) \mathrm{CN}(\mathrm{CC}(\mathrm{C})(\mathrm{C})[\mathrm{H}])[\mathrm{S}] \\
(=\mathrm{O})(=\mathrm{O}) \mathrm{C} 5=\mathrm{CC}=\mathrm{C} 4 \mathrm{OCOC} 4=\mathrm{C} 5\end{array}$ \\
\hline H98 & + & 3.876 & 4.024 & 69.339 & 4.233 & 91.913 & $\begin{array}{l}\mathrm{O}=\mathrm{C} 1 \mathrm{O}[\mathrm{C} @ @]([\mathrm{H}])(\mathrm{CN} 1 \mathrm{C}=2 \mathrm{C}=\mathrm{CC}([\mathrm{F}])=\mathrm{CC}=2) \mathrm{C}(=\mathrm{O}) \mathrm{N}[\mathrm{C} @ @ \\
]([\mathrm{H}])(\mathrm{C} / \mathrm{C} 3=\mathrm{C} / \mathrm{C}=\mathrm{CC}=\mathrm{C} 3)[\mathrm{C} @ @](\mathrm{O})([\mathrm{H}]) \mathrm{CN}(\mathrm{CC}(\mathrm{C})(\mathrm{C})[\mathrm{H}])[\mathrm{S}] \\
(=\mathrm{O})(=\mathrm{O}) \mathrm{C} 5=\mathrm{CC}=\mathrm{C} 4 \mathrm{~N}=\mathrm{C}[\mathrm{S}] \mathrm{C} 4=\mathrm{C} 5\end{array}$ \\
\hline H100 & + & 4.137 & 4.344 & 74.845 & 4.244 & 92.139 & $\begin{array}{l}\mathrm{O}=\mathrm{C} 1 \mathrm{O}[\mathrm{C} @ @]([\mathrm{H}])(\mathrm{CN} 1 \mathrm{C}=2 \mathrm{C}=\mathrm{CC}(=\mathrm{CC}=2) \mathrm{C}(\mathrm{C})=\mathrm{O}) \mathrm{C}(=\mathrm{O}) \mathrm{N}[ \\
\mathrm{C} @ @]([\mathrm{H}])(\mathrm{C} / \mathrm{C} 3=\mathrm{C} / \mathrm{C}=\mathrm{CC}=\mathrm{C} 3)[\mathrm{C} @ @](\mathrm{O})([\mathrm{H}]) \mathrm{CN}(\mathrm{CC}(\mathrm{C})(\mathrm{C})[ \\
\mathrm{H}])[\mathrm{S}](=\mathrm{O})(=\mathrm{O}) \mathrm{C} 5=\mathrm{CC}=\mathrm{C} 4 \mathrm{~N}=\mathrm{C}[\mathrm{S}] \mathrm{C} 4=\mathrm{C} 5\end{array}$ \\
\hline H101 & + & 3.499 & 3.517 & 60.600 & 3.402 & 73.875 & $\begin{array}{l}\mathrm{O}=\mathrm{C} 1 \mathrm{O}[\mathrm{C} @ @]([\mathrm{H}])(\mathrm{CN} 1 \mathrm{C}=2 \mathrm{C}=\mathrm{CC}(=\mathrm{CC}=2) \mathrm{C}(\mathrm{C})=\mathrm{O}) \mathrm{C}(=\mathrm{O}) \mathrm{N}[ \\
\mathrm{C} @ @]([\mathrm{H}])(\mathrm{C} / \mathrm{C} 3=\mathrm{C} / \mathrm{C}=\mathrm{CC}=\mathrm{C} 3)[\mathrm{C} @ @](\mathrm{O})([\mathrm{H}]) \mathrm{CN}(\mathrm{CC}(\mathrm{C})(\mathrm{C})[ \\
\mathrm{H}])[\mathrm{S}](=\mathrm{O})(=\mathrm{O}) \mathrm{C} 4=\mathrm{CC}=\mathrm{C}(\mathrm{C}=\mathrm{C} 4) \mathrm{CO}\end{array}$ \\
\hline H102 & + & 4.097 & 4.300 & 74.092 & 4.315 & 93.680 & $\begin{array}{l}\mathrm{O}=\mathrm{C} 1 \mathrm{O}[\mathrm{C} @ @]([\mathrm{H}])(\mathrm{CN} 1 \mathrm{C}=2 \mathrm{C}=\mathrm{C} / \mathrm{C}=\mathrm{C}(/[\mathrm{F}]) \mathrm{C}=2) \mathrm{C}(=\mathrm{O}) \mathrm{N}[\mathrm{C} @ \\
@]([\mathrm{H}])(\mathrm{C} / \mathrm{C} 3=\mathrm{C} / \mathrm{C}=\mathrm{CC}=\mathrm{C} 3)[\mathrm{C} @ @](\mathrm{O})([\mathrm{H}]) \mathrm{CN}(\mathrm{CC}(\mathrm{C})(\mathrm{C})[\mathrm{H}])[ \\
\mathrm{S}](=\mathrm{O})(=\mathrm{O}) \mathrm{C} 5=\mathrm{CC}=\mathrm{C} 4 \mathrm{~N}=\mathrm{C}[\mathrm{S}] \mathrm{C} 4=\mathrm{C} 5\end{array}$ \\
\hline H104 & + & 3.635 & 4.546 & 78.324 & 4.384 & 95.194 & $\begin{array}{l}\mathrm{O}=\mathrm{C} 1 \mathrm{O}[\mathrm{C} @ @]([\mathrm{H}])(\mathrm{CN} 1 \mathrm{C}=2 \mathrm{C}=\mathrm{CC}([\mathrm{F}])=\mathrm{C}([\mathrm{F}]) \mathrm{C}=2) \mathrm{C}(=\mathrm{O}) \mathrm{N}[\mathrm{C} \\
@ @]([\mathrm{H}])(\mathrm{C} / \mathrm{C} 3=\mathrm{C} / \mathrm{C}=\mathrm{CC}=\mathrm{C} 3)[\mathrm{C} @ @](\mathrm{O})([\mathrm{H}]) \mathrm{CN}(\mathrm{CC}(\mathrm{C})(\mathrm{C})[\mathrm{H} \\
])[\mathrm{S}](=\mathrm{O})(=\mathrm{O}) \mathrm{C} 5=\mathrm{CC}=\mathrm{C} 4 \mathrm{~N}=\mathrm{C}[\mathrm{S}] \mathrm{C} 4=\mathrm{C} 5\end{array}$ \\
\hline H105 & + & 3.481 & 3.719 & 64.080 & 3.543 & 76.930 & $\begin{array}{l}\mathrm{O}=\mathrm{C} 1 \mathrm{O}[\mathrm{C} @ @]([\mathrm{H}])(\mathrm{CN} 1 \mathrm{C}=2 \mathrm{C}=\mathrm{CC}([\mathrm{F}])=\mathrm{C}([\mathrm{F}]) \mathrm{C}=2) \mathrm{C}(=\mathrm{O}) \mathrm{N}[\mathrm{C} \\
@ @]([\mathrm{H}])(\mathrm{C} / \mathrm{C} 3=\mathrm{C} / \mathrm{C}=\mathrm{CC}=\mathrm{C} 3)[\mathrm{C} @ @](\mathrm{O})([\mathrm{H}]) \mathrm{CN}(\mathrm{CC}(\mathrm{C})(\mathrm{C})[\mathrm{H} \\
])[\mathrm{S}](=\mathrm{O})(=\mathrm{O}) \mathrm{C} 4=\mathrm{CC}=\mathrm{C}(\mathrm{C}=\mathrm{C} 4) \mathrm{CO}\end{array}$ \\
\hline H106 & + & 4.796 & 4.138 & 71.293 & 4.395 & 95.434 & $\begin{array}{l}\mathrm{O}=\mathrm{C} 1 \mathrm{O}[\mathrm{C} @ @]([\mathrm{H}])(\mathrm{CN} 1 \mathrm{C}=2 \mathrm{C}=\mathrm{CC}=\mathrm{C}(\mathrm{C}=2) \mathrm{C}([\mathrm{F}])([\mathrm{F}])[\mathrm{F}]) \mathrm{C}(= \\
\mathrm{O}) \mathrm{N}[\mathrm{C} @ @]([\mathrm{H}])(\mathrm{C} / \mathrm{C} 3=\mathrm{C} / \mathrm{C}=\mathrm{CC}=\mathrm{C} 3)[\mathrm{C} @ @](\mathrm{O})([\mathrm{H}]) \mathrm{CN}(\mathrm{CC}(\mathrm{C} \\
)(\mathrm{C})[\mathrm{H}])[\mathrm{S}](=\mathrm{O})(=\mathrm{O}) \mathrm{C} 5=\mathrm{CC}=\mathrm{C} 4 \mathrm{~N}=\mathrm{C}[\mathrm{S}] \mathrm{C} 4=\mathrm{C} 5\end{array}$ \\
\hline H107 & + & 3.708 & 3.311 & 57.049 & 3.554 & 77.170 & $\begin{array}{l}\mathrm{O}=\mathrm{C} 1 \mathrm{O}[\mathrm{C} @ @]([\mathrm{H}])(\mathrm{CN} 1 \mathrm{C}=2 \mathrm{C}=\mathrm{CC}=\mathrm{C}(\mathrm{C}=2) \mathrm{C}([\mathrm{F}])([\mathrm{F}])[\mathrm{F}]) \mathrm{C}(= \\
\mathrm{O}) \mathrm{N}[\mathrm{C} @ @]([\mathrm{H}])(\mathrm{C} / \mathrm{C} 3=\mathrm{C} / \mathrm{C}=\mathrm{CC}=\mathrm{C} 3)[\mathrm{C} @ @](\mathrm{O})([\mathrm{H}]) \mathrm{CN}(\mathrm{CC}(\mathrm{C} \\
)(\mathrm{C})[\mathrm{H}])[\mathrm{S}](=\mathrm{O})(=\mathrm{O}) \mathrm{C} 4=\mathrm{CC}=\mathrm{C}(\mathrm{C}=\mathrm{C} 4) \mathrm{CO}\end{array}$ \\
\hline H109 & + & 3.886 & 3.936 & 67.820 & 3.749 & 81.412 & $\begin{array}{l}\mathrm{O}=\mathrm{C} 1 \mathrm{O}[\mathrm{C} @ @]([\mathrm{H}])(\mathrm{CN} 1 \mathrm{C}=2 \mathrm{C}=\mathrm{CC}=\mathrm{C}(\mathrm{C}=2) \mathrm{OC}([\mathrm{F}])([\mathrm{F}])[\mathrm{F}]) \mathrm{C}( \\
=\mathrm{O}) \mathrm{N}[\mathrm{C} @ @]([\mathrm{H}])(\mathrm{C} / \mathrm{C} 3=\mathrm{C} / \mathrm{C}=\mathrm{CC}=\mathrm{C} 3)[\mathrm{C} @ @](\mathrm{O})([\mathrm{H}]) \mathrm{CN}(\mathrm{CC}( \\
\mathrm{C})(\mathrm{C})[\mathrm{H}])[\mathrm{S}](=\mathrm{O})(=\mathrm{O}) \mathrm{C} 4=\mathrm{CC}=\mathrm{C}(\mathrm{C}=\mathrm{C} 4) \mathrm{OC}\end{array}$ \\
\hline H110 & + & 3.622 & 3.806 & 65.574 & 3.669 & 79.659 & $\begin{array}{l}\mathrm{O}=\mathrm{C} 1 \mathrm{O}[\mathrm{C} @ @]([\mathrm{H}])(\mathrm{CN} 1 \mathrm{C}=2 \mathrm{C}=\mathrm{CC}=\mathrm{C}(\mathrm{C}=2) \mathrm{OC}([\mathrm{F}])([\mathrm{F}])[\mathrm{F}]) \mathrm{C}( \\
=\mathrm{O}) \mathrm{N}[\mathrm{C} @ @]([\mathrm{H}])(\mathrm{C} / \mathrm{C} 3=\mathrm{C} / \mathrm{C}=\mathrm{CC}=\mathrm{C} 3)[\mathrm{C} @ @](\mathrm{O})([\mathrm{H}]) \mathrm{CN}(\mathrm{CC}( \\
\mathrm{C})(\mathrm{C})[\mathrm{H}])[\mathrm{S}](=\mathrm{O})(=\mathrm{O}) \mathrm{C} 5=\mathrm{CC}=\mathrm{C} 4 \mathrm{OCOC} 4=\mathrm{C} 5\end{array}$ \\
\hline H111 & + & 4.585 & 4.182 & 72.062 & 4.084 & 88.677 & $\begin{array}{l}\mathrm{O}=\mathrm{C} 1 \mathrm{O}[\mathrm{C} @ @]([\mathrm{H}])(\mathrm{CN} 1 \mathrm{C}=2 \mathrm{C}=\mathrm{CC}=\mathrm{C}(\mathrm{C}=2) \mathrm{OC}([\mathrm{F}])([\mathrm{F}])[\mathrm{F}]) \mathrm{C}( \\
=\mathrm{O}) \mathrm{N}[\mathrm{C} @ @]([\mathrm{H}])(\mathrm{C} / \mathrm{C} 3=\mathrm{C} / \mathrm{C}=\mathrm{CC}=\mathrm{C} 3)[\mathrm{C} @ @](\mathrm{O})([\mathrm{H}]) \mathrm{CN}(\mathrm{CC}( \\
\mathrm{C})(\mathrm{C})[\mathrm{H}])[\mathrm{S}](=\mathrm{O})(=\mathrm{O}) \mathrm{C} 5=\mathrm{CC}=\mathrm{C} 4 \mathrm{~N}=\mathrm{C}[\mathrm{S}] \mathrm{C} 4=\mathrm{C} 5\end{array}$ \\
\hline H113 & + & 4.824 & 4.968 & 85.588 & 4.958 & 107.649 & $\begin{array}{l}\mathrm{O}=\mathrm{C} 1 \mathrm{O}[\mathrm{C} @ @]([\mathrm{H}])(\mathrm{CN} 1 \mathrm{C}=2 \mathrm{C}=\mathrm{CC}=\mathrm{C}(\mathrm{C}=2) \mathrm{C}(\mathrm{C})=\mathrm{O}) \mathrm{C}(=\mathrm{O}) \mathrm{N}[ \\
\mathrm{C} @ @]([\mathrm{H}])(\mathrm{C} / \mathrm{C} 3=\mathrm{C} / \mathrm{C}=\mathrm{CC}=\mathrm{C} 3)[\mathrm{C} @ @](\mathrm{O})([\mathrm{H}]) \mathrm{CN}(\mathrm{CC}(\mathrm{C})(\mathrm{C})[ \\
\mathrm{H}])[\mathrm{S}](=\mathrm{O})(=\mathrm{O}) \mathrm{C} 5=\mathrm{CC}=\mathrm{C} 4 \mathrm{~N}=\mathrm{C}[\mathrm{S}] \mathrm{C} 4=\mathrm{C} 5\end{array}$ \\
\hline H114 & + & 3.627 & 4.141 & 71.344 & 4.117 & 89.385 & $\begin{array}{l}\mathrm{O}=\mathrm{C} 1 \mathrm{O}[\mathrm{C} @ @]([\mathrm{H}])(\mathrm{CN} 1 \mathrm{C}=2 \mathrm{C}=\mathrm{CC}=\mathrm{C}(\mathrm{C}=2) \mathrm{C}(\mathrm{C})=\mathrm{O}) \mathrm{C}(=\mathrm{O}) \mathrm{N}[ \\
\mathrm{C} @ @]([\mathrm{H}])(\mathrm{C} / \mathrm{C} 3=\mathrm{C} / \mathrm{C}=\mathrm{CC}=\mathrm{C} 3)[\mathrm{C} @ @](\mathrm{O})([\mathrm{H}]) \mathrm{CN}(\mathrm{CC}(\mathrm{C})(\mathrm{C})[ \\
\mathrm{H}])[\mathrm{S}](=\mathrm{O})(=\mathrm{O}) \mathrm{C} 4=\mathrm{CC}=\mathrm{C}(\mathrm{C}=\mathrm{C} 4) \mathrm{CO}\end{array}$ \\
\hline H116 & + & 4.602 & 4.387 & 75.594 & 4.474 & 97.147 & $\begin{array}{l}\mathrm{O}=\mathrm{C} 1 \mathrm{O}[\mathrm{C} @ @]([\mathrm{H}])(\mathrm{CN} 1 \mathrm{C}=2 \mathrm{C}=\mathrm{CC}=\mathrm{C}(\mathrm{C}=2)[\mathrm{S}](\mathrm{C})(=\mathrm{O})=\mathrm{O}) \mathrm{C}(= \\
\mathrm{O}) \mathrm{N}[\mathrm{C} @ @]([\mathrm{H}])(\mathrm{C} / \mathrm{C} 3=\mathrm{C} / \mathrm{C}=\mathrm{CC}=\mathrm{C} 3)[\mathrm{C} @ @](\mathrm{O})([\mathrm{H}]) \mathrm{CN}(\mathrm{CC}(\mathrm{C} \\
)(\mathrm{C})[\mathrm{H}])[\mathrm{S}](=\mathrm{O})(=\mathrm{O}) \mathrm{C} 5=\mathrm{CC}=\mathrm{C} 4 \mathrm{OCOC} 4=\mathrm{C} 5\end{array}$ \\
\hline
\end{tabular}




\begin{tabular}{|c|c|c|c|c|c|c|c|}
\hline H118 & + & 3.866 & 4.316 & 74.364 & 4.279 & 92.905 & $\begin{array}{l}\mathrm{O}=\mathrm{C} 1 \mathrm{O}[\mathrm{C} @ @]([\mathrm{H}])(\mathrm{CN} 1 \mathrm{C}=2 \mathrm{C}=\mathrm{CC}=\mathrm{C}(\mathrm{C}=2)[\mathrm{N}+](=\mathrm{O})[\mathrm{O}- \\
]) \mathrm{C}(=\mathrm{O}) \mathrm{N}[\mathrm{C} @ @]([\mathrm{H}])(\mathrm{C} / \mathrm{C} 3=\mathrm{C} / \mathrm{C}=\mathrm{CC}=\mathrm{C} 3)[\mathrm{C} @ @](\mathrm{O})([\mathrm{H}]) \mathrm{CN}( \\
\mathrm{CC}(\mathrm{C})(\mathrm{C})[\mathrm{H}])[\mathrm{S}](=\mathrm{O})(=\mathrm{O}) \mathrm{C} 4=\mathrm{CC}=\mathrm{C}(\mathrm{C}=\mathrm{C} 4) \mathrm{OC}\end{array}$ \\
\hline H119 & + & 3.932 & 4.186 & 72.118 & 4.198 & 91.151 & $\begin{array}{l}\mathrm{O}=\mathrm{C} 1 \mathrm{O}[\mathrm{C} @ @]([\mathrm{H}])(\mathrm{CN} 1 \mathrm{C}=2 \mathrm{C}=\mathrm{CC}=\mathrm{C}(\mathrm{C}=2)[\mathrm{N}+](=\mathrm{O})[\mathrm{O}- \\
]) \mathrm{C}(=\mathrm{O}) \mathrm{N}[\mathrm{C} @ @]([\mathrm{H}])(\mathrm{C} / \mathrm{C} 3=\mathrm{C} / \mathrm{C}=\mathrm{CC}=\mathrm{C} 3)[\mathrm{C} @ @](\mathrm{O})([\mathrm{H}]) \mathrm{CN}( \\
\mathrm{CC}(\mathrm{C})(\mathrm{C})[\mathrm{H}])[\mathrm{S}](=\mathrm{O})(=\mathrm{O}) \mathrm{C} 5=\mathrm{CC}=\mathrm{C} 4 \mathrm{OCOC} 4=\mathrm{C} 5\end{array}$ \\
\hline H121 & + & 4.699 & 4.796 & 82.634 & 4.750 & 103.139 & $\begin{array}{l}\mathrm{O}=\mathrm{C} 1 \mathrm{O}[\mathrm{C} @ @]([\mathrm{H}])(\mathrm{CN} 1 \mathrm{C}=2 \mathrm{C}=\mathrm{C} / \mathrm{C}=\mathrm{C}(/ \mathrm{N}) \mathrm{C}=2) \mathrm{C}(=\mathrm{O}) \mathrm{N}[\mathrm{C} @ @ \\
]([\mathrm{H}])(\mathrm{C} / \mathrm{C} 3=\mathrm{C} / \mathrm{C}=\mathrm{CC}=\mathrm{C} 3)[\mathrm{C} @ @](\mathrm{O})([\mathrm{H}]) \mathrm{CN}(\mathrm{CC}(\mathrm{C})(\mathrm{C})[\mathrm{H}])[\mathrm{S}] \\
(=\mathrm{O})(=\mathrm{O}) \mathrm{C} 4=\mathrm{CC}=\mathrm{C}(\mathrm{C}=\mathrm{C} 4) \mathrm{OC}\end{array}$ \\
\hline H123 & + & 5.097 & 5.042 & 86.876 & 5.085 & 110.404 & $\begin{array}{l}\mathrm{O}=\mathrm{C} 1 \mathrm{O}[\mathrm{C} @ @]([\mathrm{H}])(\mathrm{CN} 1 \mathrm{C}=2 \mathrm{C}=\mathrm{C} / \mathrm{C}=\mathrm{C}(/ \mathrm{N}) \mathrm{C}=2) \mathrm{C}(=\mathrm{O}) \mathrm{N}[\mathrm{C} @ @ @ \\
]([\mathrm{H}])(\mathrm{C} / \mathrm{C} 3=\mathrm{C} / \mathrm{C}=\mathrm{CC}=\mathrm{C} 3)[\mathrm{C} @ @](\mathrm{O})([\mathrm{H}]) \mathrm{CN}(\mathrm{CC}(\mathrm{C})(\mathrm{C})[\mathrm{H}])[\mathrm{S}] \\
(=\mathrm{O})(=\mathrm{O}) \mathrm{C} 5=\mathrm{CC}=\mathrm{C} 4 \mathrm{~N}=\mathrm{C}[\mathrm{S}] \mathrm{C} 4=\mathrm{C} 5\end{array}$ \\
\hline H124 & + & 3.914 & 4.069 & 70.104 & 4.036 & 87.644 & $\begin{array}{l}\mathrm{O}=\mathrm{C} 1 \mathrm{O}[\mathrm{C} @ @]([\mathrm{H}])(\mathrm{CN} 1 \mathrm{C}=2 \mathrm{C}=\mathrm{CC}=\mathrm{C}(\mathrm{C}=2) \mathrm{NC}(=\mathrm{O}) \mathrm{C}) \mathrm{C}(=\mathrm{O}) \mathrm{N} \\
{[\mathrm{C} @ @]([\mathrm{H}])(\mathrm{C} / \mathrm{C} 3=\mathrm{C} / \mathrm{C}=\mathrm{CC}=\mathrm{C} 3)[\mathrm{C} @ @](\mathrm{O})([\mathrm{H}]) \mathrm{CN}(\mathrm{CC}(\mathrm{C})(\mathrm{C})} \\
\mathrm{H}])[\mathrm{S}](=\mathrm{O})(=\mathrm{O}) \mathrm{C} 4=\mathrm{CC}=\mathrm{C}(\mathrm{C}=\mathrm{C} 4) \mathrm{OC}\end{array}$ \\
\hline H127 & + & 3.788 & 3.867 & 66.630 & 3.898 & 84.635 & $\begin{array}{l}\mathrm{O}=\mathrm{C} 1 \mathrm{O}[\mathrm{C} @ @]([\mathrm{H}])(\mathrm{CN} 1 \mathrm{C}=2 \mathrm{C}=\mathrm{CC}=\mathrm{C}(\mathrm{C}=2) \mathrm{NC}(=\mathrm{O}) \mathrm{OC}) \mathrm{C}(=\mathrm{O}) \\
\mathrm{N}[\mathrm{C} @ @]([\mathrm{H}])(\mathrm{C} / \mathrm{C} 3=\mathrm{C} / \mathrm{C}=\mathrm{CC}=\mathrm{C} 3)[\mathrm{C} @ @](\mathrm{O})([\mathrm{H}]) \mathrm{CN}(\mathrm{CC}(\mathrm{C})( \\
\mathrm{C})[\mathrm{H}])[\mathrm{S}](=\mathrm{O})(=\mathrm{O}) \mathrm{C} 4=\mathrm{CC}=\mathrm{C}(\mathrm{C}=\mathrm{C} 4) \mathrm{OC}\end{array}$ \\
\hline H129 & + & 4.585 & 4.113 & 70.872 & 4.233 & 91.900 & $\begin{array}{l}\mathrm{O}=\mathrm{C} 1 \mathrm{O}[\mathrm{C} @ @]([\mathrm{H}])(\mathrm{CN} 1 \mathrm{C}=2 \mathrm{C}=\mathrm{CC}=\mathrm{C}(\mathrm{C}=2) \mathrm{NC}(=\mathrm{O}) \mathrm{OC}) \mathrm{C}(=\mathrm{O}) \\
\mathrm{N}[\mathrm{C} @ @]([\mathrm{H}])(\mathrm{C} / \mathrm{C} 3=\mathrm{C} / \mathrm{C}=\mathrm{CC}=\mathrm{C} 3)[\mathrm{C} @ @](\mathrm{O})([\mathrm{H}]) \mathrm{CN}(\mathrm{CC}(\mathrm{C})( \\
\mathrm{C})[\mathrm{H}])[\mathrm{S}](=\mathrm{O})(=\mathrm{O}) \mathrm{C} 5=\mathrm{CC}=\mathrm{C} 4 \mathrm{~N}=\mathrm{C}[\mathrm{S}] \mathrm{C} 4=\mathrm{C} 5\end{array}$ \\
\hline $\mathrm{H} 3$ & - & 1.923 & 1.947 & 33.537 & 1.884 & 40.924 & $\begin{array}{l}\mathrm{O}=\mathrm{C}(\mathrm{OCCNC}(=\mathrm{O}) \mathrm{C}([\mathrm{F}])([\mathrm{F}])[\mathrm{F}]) \mathrm{N}[\mathrm{C} @ @]([\mathrm{H}])(\mathrm{C} / \mathrm{C} 1=\mathrm{C} / \mathrm{C}=\mathrm{CC} \\
=\mathrm{C} 1)[\mathrm{C} @](\mathrm{O})([\mathrm{H}]) \mathrm{CN}(\mathrm{C}[\mathrm{C} @ @](\mathrm{C})([\mathrm{H}]) \mathrm{CC})[\mathrm{S}](=\mathrm{O})(=\mathrm{O}) \mathrm{C} 2=\mathrm{C} \\
\mathrm{C}=\mathrm{C}(\mathrm{C}=\mathrm{C} 2) \mathrm{OC}\end{array}$ \\
\hline $\mathrm{H} 7$ & - & 4.553 & 4.419 & 76.137 & 4.544 & 98.663 & $\begin{array}{l}\mathrm{O}=\mathrm{C}(\mathrm{OC}[\mathrm{C} @ @](\mathrm{C})([\mathrm{H}]) \mathrm{C}(=\mathrm{O}) \mathrm{OC}) \mathrm{N}[\mathrm{C} @ @]([\mathrm{H}])(\mathrm{C} / \mathrm{C} 1=\mathrm{C} / \mathrm{C}=\mathrm{C} \\
\mathrm{C}=\mathrm{C} 1)[\mathrm{C} @](\mathrm{O})([\mathrm{H}]) \mathrm{CN}(\mathrm{C}[\mathrm{C} @ @](\mathrm{C})([\mathrm{H}]) \mathrm{CC})[\mathrm{S}](=\mathrm{O})(=\mathrm{O}) \mathrm{C} 2= \\
\mathrm{CC}=\mathrm{C}(\mathrm{C}=\mathrm{C} 2) \mathrm{OC}\end{array}$ \\
\hline H10 & - & 1.455 & 1.372 & 23.637 & 1.410 & 30.632 & $\begin{array}{l}\mathrm{O}=\mathrm{C}(\mathrm{O}[\mathrm{C} @] 1([\mathrm{H}]) \mathrm{CCOC} 1=\mathrm{O}) \mathrm{N}[\mathrm{C} @ @]([\mathrm{H}])(\mathrm{C} / \mathrm{C} 2=\mathrm{C} / \mathrm{C}=\mathrm{CC}=\mathrm{C} \\
2)[\mathrm{C} @](\mathrm{O})([\mathrm{H}]) \mathrm{CN}(\mathrm{C}[\mathrm{C} @ @](\mathrm{C})([\mathrm{H}]) \mathrm{CC})[\mathrm{S}](=\mathrm{O})(=\mathrm{O}) \mathrm{C} 3=\mathrm{CC}= \\
\mathrm{C}(\mathrm{C}=\mathrm{C} 3) \mathrm{OC}\end{array}$ \\
\hline $\mathrm{H} 20$ & - & 0.915 & 1.591 & 27.403 & 1.675 & 36.373 & $\begin{array}{l}\mathrm{O}=\mathrm{C}(\mathrm{O}[\mathrm{C} @] 1([\mathrm{H}]) \mathrm{CCOC} 1=\mathrm{O}) \mathrm{N}[\mathrm{C} @ @]([\mathrm{H}])(\mathrm{C} / \mathrm{C} 2=\mathrm{C} / \mathrm{C}=\mathrm{CC}=\mathrm{C} \\
2)[\mathrm{C} @](\mathrm{O})([\mathrm{H}]) \mathrm{CN}(\mathrm{C}[\mathrm{C} @ @](\mathrm{C})([\mathrm{H}]) \mathrm{CC})[\mathrm{S}](=\mathrm{O})(=\mathrm{O}) \mathrm{C} 4=\mathrm{CC}= \\
\mathrm{C} 3 \mathrm{~N}=\mathrm{C}[\mathrm{S}] \mathrm{C} 3=\mathrm{C} 4\end{array}$ \\
\hline $\mathrm{H} 25$ & - & 2.529 & 2.690 & 46.355 & 2.746 & 59.637 & $\begin{array}{l}\mathrm{O}=\mathrm{C}(\mathrm{OCCNC}(=\mathrm{O}) \mathrm{OC}(\mathrm{C})(\mathrm{C}) \mathrm{C}) \mathrm{N}[\mathrm{C} @ @]([\mathrm{H}])(\mathrm{C} / \mathrm{C} 1=\mathrm{C} / \mathrm{C}=\mathrm{CC}=\mathrm{C} \\
1)[\mathrm{C} @](\mathrm{O})([\mathrm{H}]) \mathrm{CN}(\mathrm{CCCCC})[\mathrm{S}](=\mathrm{O})(=\mathrm{O}) \mathrm{C} 2=\mathrm{CC}=\mathrm{C}(\mathrm{C}=\mathrm{C} 2) \mathrm{OC}\end{array}$ \\
\hline $\mathrm{H} 29$ & - & 1.181 & 0.806 & 13.888 & 0.833 & 18.113 & $\begin{array}{l}\mathrm{O}=\mathrm{C}(\mathrm{O}[\mathrm{C} @] 1([\mathrm{H}]) \mathrm{CCOC} 1=\mathrm{O}) \mathrm{N}[\mathrm{C} @ @]([\mathrm{H}])(\mathrm{C} / \mathrm{C} 2=\mathrm{C} / \mathrm{C}=\mathrm{CC}=\mathrm{C} \\
2)[\mathrm{C} @](\mathrm{O})([\mathrm{H}]) \mathrm{CN}(\mathrm{CCCCC})[\mathrm{S}](=\mathrm{O})(=\mathrm{O}) \mathrm{C} 3=\mathrm{CC}=\mathrm{C}(\mathrm{C}=\mathrm{C} 3) \mathrm{OC}\end{array}$ \\
\hline H34 & - & 2.472 & 2.633 & 45.365 & 2.678 & 58.157 & $\begin{array}{l}\mathrm{O}=\mathrm{C}(\mathrm{O}[\mathrm{C} @ @](\mathrm{C})([\mathrm{H}]) \mathrm{CCC}(\mathrm{C})=\mathrm{O}) \mathrm{N}[\mathrm{C} @ @]([\mathrm{H}])(\mathrm{C} / \mathrm{C} 1=\mathrm{C} / \mathrm{C}=\mathrm{C} \\
\mathrm{C}=\mathrm{C} 1)[\mathrm{C} @](\mathrm{O})([\mathrm{H}]) \mathrm{CN}(\mathrm{CCCCC})[\mathrm{S}](=\mathrm{O})(=\mathrm{O}) \mathrm{C} 3=\mathrm{CC}=\mathrm{C} 2 \mathrm{~N}=\mathrm{C}[ \\
\mathrm{S}] \mathrm{C} 2=\mathrm{C} 3\end{array}$ \\
\hline $\mathrm{H} 47$ & - & 4.347 & 4.447 & 76.622 & 4.761 & 103.379 & $\begin{array}{l}\mathrm{O}=\mathrm{C} 1 \mathrm{O}[\mathrm{C} @ @]([\mathrm{H}])(\mathrm{CN} 1 \mathrm{C}=2 \mathrm{C}=\mathrm{CC}=\mathrm{C}(\mathrm{C}=2) \mathrm{OC}) \mathrm{C}(=\mathrm{O}) \mathrm{N}[\mathrm{C} @ @ @ \\
]([\mathrm{H}])(\mathrm{C} / \mathrm{C} 3=\mathrm{C} / \mathrm{C}=\mathrm{CC}=\mathrm{C} 3)[\mathrm{C} @ @](\mathrm{O})([\mathrm{H}]) \mathrm{CN}(\mathrm{CC}(\mathrm{C})(\mathrm{C})[\mathrm{H}])[\mathrm{S}] \\
(=\mathrm{O})(=\mathrm{O}) \mathrm{C} 4=\mathrm{CC}=\mathrm{C}(\mathrm{C}=\mathrm{C} 4) \mathrm{OC}\end{array}$ \\
\hline H50 & - & 3.638 & 3.850 & 66.341 & 3.693 & 80.196 & $\begin{array}{l}\mathrm{O}=\mathrm{C} 1 \mathrm{O}[\mathrm{C} @ @]([\mathrm{H}])(\mathrm{CN} 1 \mathrm{C}=2 \mathrm{C}=\mathrm{CC}([\mathrm{F}])=\mathrm{C}([\mathrm{F}]) \mathrm{C}=2) \mathrm{C}(=\mathrm{O}) \mathrm{N}[\mathrm{C} \\
@ @]([\mathrm{H}])(\mathrm{C} / \mathrm{C} 3=\mathrm{C} / \mathrm{C}=\mathrm{CC}=\mathrm{C} 3)[\mathrm{C} @ @](\mathrm{O})([\mathrm{H}]) \mathrm{CN}(\mathrm{CC}(\mathrm{C})(\mathrm{C})[\mathrm{H} \\
])[\mathrm{S}](=\mathrm{O})(=\mathrm{O}) \mathrm{C} 4=\mathrm{C} / \mathrm{C}=\mathrm{C}(/ \mathrm{N}) \mathrm{C}=\mathrm{C} 4\end{array}$ \\
\hline H52 & - & 3.495 & 4.272 & 73.606 & 4.267 & 92.651 & $\begin{array}{l}\mathrm{O}=\mathrm{C} 1 \mathrm{O}[\mathrm{C} @ @]([\mathrm{H}])(\mathrm{CN} 1 \mathrm{C}=2 \mathrm{C}=\mathrm{CC}=\mathrm{C}(\mathrm{C}=2) \mathrm{C}(=\mathrm{O}) \mathrm{C}) \mathrm{C}(=\mathrm{O}) \mathrm{N}[ \\
\mathrm{C} @ @]([\mathrm{H}])(\mathrm{C} / \mathrm{C} 3=\mathrm{C} / \mathrm{C}=\mathrm{CC}=\mathrm{C} 3)[\mathrm{C} @ @](\mathrm{O})([\mathrm{H}]) \mathrm{CN}(\mathrm{CC}(\mathrm{C})(\mathrm{C})[ \\
\mathrm{H}])[\mathrm{S}](=\mathrm{O})(=\mathrm{O}) \mathrm{C} 4=\mathrm{C} / \mathrm{C}=\mathrm{C}(/ \mathrm{N}) \mathrm{C}=\mathrm{C} 4\end{array}$ \\
\hline
\end{tabular}




\begin{tabular}{|c|c|c|c|c|c|c|c|}
\hline H61 & - & 4.143 & 3.877 & 66.807 & 4.096 & 88.940 & $\begin{array}{l}\mathrm{O}=\mathrm{C} 1 \mathrm{O}[\mathrm{C} @ @]([\mathrm{H}])(\mathrm{CN} 1 \mathrm{C}=2 \mathrm{C}=\mathrm{CC}=\mathrm{C}(\mathrm{C}=2) \mathrm{C}([\mathrm{F}])([\mathrm{F}])[\mathrm{F}]) \mathrm{C}(= \\
\mathrm{O}) \mathrm{N}[\mathrm{C} @ @]([\mathrm{H}])(\mathrm{C} / \mathrm{C} 3=\mathrm{C} / \mathrm{C}=\mathrm{CC}=\mathrm{C} 3)[\mathrm{C} @ @](\mathrm{O})([\mathrm{H}]) \mathrm{CN}(\mathrm{CC}(\mathrm{C} \\
)(\mathrm{C})[\mathrm{H}])[\mathrm{S}](=\mathrm{O})(=\mathrm{O}) \mathrm{C} 4=\mathrm{C} / \mathrm{C}=\mathrm{C}(/ \mathrm{OC}) \mathrm{C}(\mathrm{C})=\mathrm{C} 4\end{array}$ \\
\hline H64 & - & 2.000 & 2.336 & 40.249 & 2.196 & 47.697 & $\begin{array}{l}\mathrm{O}=\mathrm{C} 1 \mathrm{O}[\mathrm{C} @ @]([\mathrm{H}])(\mathrm{CN} 1 \mathrm{C}=2 \mathrm{C}=\mathrm{CC}=\mathrm{C}(\mathrm{C}=2) \mathrm{C}([\mathrm{F}])([\mathrm{F}])[\mathrm{F}]) \mathrm{C}(= \\
\mathrm{O}) \mathrm{N}[\mathrm{C} @ @]([\mathrm{H}])(\mathrm{C} / \mathrm{C} 3=\mathrm{C} / \mathrm{C}=\mathrm{CC}=\mathrm{C} 3)[\mathrm{C} @ @](\mathrm{O})([\mathrm{H}]) \mathrm{CN}(\mathrm{CC}(\mathrm{C} \\
)(\mathrm{C})[\mathrm{H}])[\mathrm{S}](=\mathrm{O})(=\mathrm{O}) \mathrm{C} 4=\mathrm{CC}=\mathrm{C}(\mathrm{C}=\mathrm{C} 4) \mathrm{OC}([\mathrm{F}])([\mathrm{F}])[\mathrm{F}] \sim\end{array}$ \\
\hline H68 & - & 3.590 & 3.460 & 59.610 & 3.564 & 77.392 & $\begin{array}{l}\mathrm{O}=\mathrm{C} 1 \mathrm{O}[\mathrm{C} @ @]([\mathrm{H}])(\mathrm{CN} 1 \mathrm{C}=2 \mathrm{C}=\mathrm{C} / \mathrm{C}=\mathrm{C}(/[\mathrm{F}]) \mathrm{C}=2) \mathrm{C}(=\mathrm{O}) \mathrm{N}[\mathrm{C} @ \\
@]([\mathrm{H}])(\mathrm{C} / \mathrm{C} 3=\mathrm{C} / \mathrm{C}=\mathrm{CC}=\mathrm{C} 3)[\mathrm{C} @ @](\mathrm{O})([\mathrm{H}]) \mathrm{CN}(\mathrm{C}[\mathrm{C} @ @] 4([\mathrm{H}] \\
) \mathrm{CC} 4)[\mathrm{S}](=\mathrm{O})(=\mathrm{O}) \mathrm{C} 5=\mathrm{CC}=\mathrm{C}(\mathrm{C}=\mathrm{C} 5) \mathrm{OC}\end{array}$ \\
\hline $\mathrm{H} 74$ & - & 0.769 & 0.745 & 12.839 & 0.789 & 17.155 & $\begin{array}{l}\mathrm{O}=\mathrm{C} 1 \mathrm{O}[\mathrm{C} @ @]([\mathrm{H}])(\mathrm{CN} 1 \mathrm{C}=2 \mathrm{C}=\mathrm{CC}=\mathrm{CC}=2) \mathrm{C}(=\mathrm{O}) \mathrm{N}[\mathrm{C} @ @]([\mathrm{H}] \\
)(\mathrm{C} / \mathrm{C} 3=\mathrm{C} / \mathrm{C}=\mathrm{CC}=\mathrm{C} 3)[\mathrm{C} @ @](\mathrm{O})([\mathrm{H}]) \mathrm{CN}(\mathrm{C} / \mathrm{C} 4=\mathrm{C} / \mathrm{C}=\mathrm{C}[\mathrm{S}] 4)[\mathrm{S}]( \\
=\mathrm{O})(=\mathrm{O}) \mathrm{C} 5=\mathrm{CC}([\mathrm{F}])=\mathrm{C}([\mathrm{F}]) \mathrm{C}=\mathrm{C} 5[\mathrm{~F}]\end{array}$ \\
\hline H82 & - & 2.133 & 2.067 & 35.613 & 2.147 & 46.624 & $\begin{array}{l}\mathrm{O}=\mathrm{C} 1 \mathrm{O}[\mathrm{C} @ @]([\mathrm{H}])(\mathrm{CN} 1 \mathrm{C}=2 \mathrm{C}=\mathrm{CC}=\mathrm{CC}=2 \mathrm{O}) \mathrm{C}(=\mathrm{O}) \mathrm{N}[\mathrm{C} @ @]([ \\
\mathrm{H}])(\mathrm{C} / \mathrm{C} 3=\mathrm{C} / \mathrm{C}=\mathrm{CC}=\mathrm{C} 3)[\mathrm{C} @ @](\mathrm{O})([\mathrm{H}]) \mathrm{CN}(\mathrm{CC}(\mathrm{C})(\mathrm{C})[\mathrm{H}])[\mathrm{S}](= \\
\mathrm{O})(=\mathrm{O}) \mathrm{C} 4=\mathrm{C} / \mathrm{C}=\mathrm{C}(/ \mathrm{N}) \mathrm{C}=\mathrm{C} 4\end{array}$ \\
\hline H83 & - & 4.013 & 4.371 & 75.308 & 4.383 & 95.157 & $\begin{array}{l}\mathrm{O}=\mathrm{C} 1 \mathrm{O}[\mathrm{C} @ @]([\mathrm{H}])(\mathrm{CN} 1 \mathrm{C}=2 \mathrm{C}=\mathrm{CC}=\mathrm{CC}=2 \mathrm{C}([\mathrm{F}])([\mathrm{F}])[\mathrm{F}]) \mathrm{C}(=\mathrm{O} \\
) \mathrm{N}[\mathrm{C} @ @]([\mathrm{H}])(\mathrm{C} / \mathrm{C} 3=\mathrm{C} / \mathrm{C}=\mathrm{CC}=\mathrm{C} 3)[\mathrm{C} @ @](\mathrm{O})([\mathrm{H}]) \mathrm{CN}(\mathrm{CC}(\mathrm{C})( \\
\mathrm{C})[\mathrm{H}])[\mathrm{S}](=\mathrm{O})(=\mathrm{O}) \mathrm{C} 5=\mathrm{CC}=\mathrm{C} 4 \mathrm{~N}=\mathrm{C}[\mathrm{S}] \mathrm{C} 4=\mathrm{C} 5\end{array}$ \\
\hline H96 & - & 3.893 & 3.778 & 65.097 & 3.899 & 84.648 & $\begin{array}{l}\mathrm{O}=\mathrm{C} 1 \mathrm{O}[\mathrm{C} @ @]([\mathrm{H}])(\mathrm{CN} 1 \mathrm{C}=2 \mathrm{C}=\mathrm{CC}([\mathrm{F}])=\mathrm{CC}=2) \mathrm{C}(=\mathrm{O}) \mathrm{N}[\mathrm{C} @ @ \\
]([\mathrm{H}])(\mathrm{C} / \mathrm{C} 3=\mathrm{C} / \mathrm{C}=\mathrm{CC}=\mathrm{C} 3)[\mathrm{C} @ @](\mathrm{O})([\mathrm{H}]) \mathrm{CN}(\mathrm{CC}(\mathrm{C})(\mathrm{C})[\mathrm{H}])[\mathrm{S}] \\
(=\mathrm{O})(=\mathrm{O}) \mathrm{C} 4=\mathrm{CC}=\mathrm{C}(\mathrm{C}=\mathrm{C} 4) \mathrm{OC}\end{array}$ \\
\hline H115 & - & 4.310 & 4.518 & 77.840 & 4.555 & 98.900 & $\begin{array}{l}\mathrm{O}=\mathrm{C} 1 \mathrm{O}[\mathrm{C} @ @]([\mathrm{H}])(\mathrm{CN} 1 \mathrm{C}=2 \mathrm{C}=\mathrm{CC}=\mathrm{C}(\mathrm{C}=2)[\mathrm{S}](\mathrm{C})(=\mathrm{O})=\mathrm{O}) \mathrm{C}(= \\
\mathrm{O}) \mathrm{N}[\mathrm{C} @ @]([\mathrm{H}])(\mathrm{C} / \mathrm{C} 3=\mathrm{C} / \mathrm{C}=\mathrm{CC}=\mathrm{C} 3)[\mathrm{C} @ @](\mathrm{O})([\mathrm{H}]) \mathrm{CN}(\mathrm{CC}(\mathrm{C} \\
)(\mathrm{C})[\mathrm{H}])[\mathrm{S}](=\mathrm{O})(=\mathrm{O}) \mathrm{C} 4=\mathrm{CC}=\mathrm{C}(\mathrm{C}=\mathrm{C} 4) \mathrm{OC}\end{array}$ \\
\hline H120 & - & 4.824 & 4.562 & 78.605 & 4.614 & 100.169 & $\begin{array}{l}\mathrm{O}=\mathrm{C} 1 \mathrm{O}[\mathrm{C} @ @]([\mathrm{H}])(\mathrm{CN} 1 \mathrm{C}=2 \mathrm{C}=\mathrm{CC}=\mathrm{C}(\mathrm{C}=2)[\mathrm{N}+](=\mathrm{O})[\mathrm{O}- \\
]) \mathrm{C}(=\mathrm{O}) \mathrm{N}[\mathrm{C} @ @]([\mathrm{H}])(\mathrm{C} / \mathrm{C} 3=\mathrm{C} / \mathrm{C}=\mathrm{CC}=\mathrm{C} 3)[\mathrm{C} @ @](\mathrm{O})([\mathrm{H}]) \mathrm{CN}( \\
\mathrm{CC}(\mathrm{C})(\mathrm{C})[\mathrm{H}])[\mathrm{S}](=\mathrm{O})(=\mathrm{O}) \mathrm{C} 5=\mathrm{CC}=\mathrm{C} 4 \mathrm{~N}=\mathrm{C}[\mathrm{S}] \mathrm{C} 4=\mathrm{C} 5\end{array}$ \\
\hline H128 & - & 3.539 & 3.737 & 64.384 & 3.817 & 82.882 & $\begin{array}{l}\mathrm{O}=\mathrm{C} 1 \mathrm{O}[\mathrm{C} @ @]([\mathrm{H}])(\mathrm{CN} 1 \mathrm{C}=2 \mathrm{C}=\mathrm{CC}=\mathrm{C}(\mathrm{C}=2) \mathrm{NC}(=\mathrm{O}) \mathrm{OC}) \mathrm{C}(=\mathrm{O}) \\
\mathrm{N}[\mathrm{C} @ @]([\mathrm{H}])(\mathrm{C} / \mathrm{C} 3=\mathrm{C} / \mathrm{C}=\mathrm{CC}=\mathrm{C} 3)[\mathrm{C} @ @](\mathrm{O})([\mathrm{H}]) \mathrm{CN}(\mathrm{CC}(\mathrm{C})( \\
\mathrm{C})[\mathrm{H}])[\mathrm{S}](=\mathrm{O})(=\mathrm{O}) \mathrm{C} 5=\mathrm{CC}=\mathrm{C} 4 \mathrm{OCOC} 4=\mathrm{C} 5\end{array}$ \\
\hline H1 & $\#$ & 3.614 & 2.893 & 49.841 & 3.750 & 81.431 & $\begin{array}{l}\mathrm{O}=\mathrm{C}(\mathrm{OC}(\mathrm{C})(\mathrm{C}) \mathrm{C}) \mathrm{N}[\mathrm{C} @ @]([\mathrm{H}])(\mathrm{C} / \mathrm{C} 1=\mathrm{C} / \mathrm{C}=\mathrm{CC}=\mathrm{C} 1)[\mathrm{C} @](\mathrm{O})([ \\
\mathrm{H}]) \mathrm{CN}(\mathrm{C}[\mathrm{C} @ @](\mathrm{C})([\mathrm{H}]) \mathrm{CC})[\mathrm{S}](=\mathrm{O})(=\mathrm{O}) \mathrm{C} 3=\mathrm{CC}=\mathrm{C} 2 \mathrm{~N}=\mathrm{C}[\mathrm{S}] \mathrm{C} 2 \\
=\mathrm{C} 3\end{array}$ \\
\hline H9 & \# & 5.523 & 5.550 & 95.623 & 5.613 & 121.860 & $\begin{array}{l}\mathrm{O}=\mathrm{C}(\mathrm{OCCN} 1 \mathrm{CCOC} 1=\mathrm{O}) \mathrm{N}[\mathrm{C} @ @]([\mathrm{H}])(\mathrm{C} / \mathrm{C} 2=\mathrm{C} / \mathrm{C}=\mathrm{CC}=\mathrm{C} 2)[\mathrm{C} \\
@](\mathrm{O})([\mathrm{H}]) \mathrm{CN}(\mathrm{C}[\mathrm{C} @ @](\mathrm{C})([\mathrm{H}]) \mathrm{CC})[\mathrm{S}](=\mathrm{O})(=\mathrm{O}) \mathrm{C} 3=\mathrm{CC}=\mathrm{C}(\mathrm{C}= \\
\mathrm{C} 3) \mathrm{OC}\end{array}$ \\
\hline H13 & \# & 2.040 & 2.019 & 34.786 & 1.942 & 42.175 & $\begin{array}{l}\mathrm{O}=\mathrm{C}(\mathrm{OCCNC}(=\mathrm{O}) \mathrm{C}([\mathrm{F}])([\mathrm{F}])[\mathrm{F}]) \mathrm{N}[\mathrm{C} @ @]([\mathrm{H}])(\mathrm{C} / \mathrm{C} 1=\mathrm{C} / \mathrm{C}=\mathrm{CC} \\
=\mathrm{C} 1)[\mathrm{C} @](\mathrm{O})([\mathrm{H}]) \mathrm{CN}(\mathrm{C}[\mathrm{C} @ @](\mathrm{C})([\mathrm{H}]) \mathrm{CC})[\mathrm{S}](=\mathrm{O})(=\mathrm{O}) \mathrm{C} 3=\mathrm{C} \\
\mathrm{C}=\mathrm{C} 2 \mathrm{~N}=\mathrm{C}[\mathrm{S}] \mathrm{C} 2=\mathrm{C} 3\end{array}$ \\
\hline H17 & \# & 2.856 & 3.752 & 64.647 & 3.714 & 80.652 & $\begin{array}{l}\mathrm{O}=\mathrm{C}(\mathrm{OC}[\mathrm{C} @](\mathrm{C})([\mathrm{H}]) \mathrm{C}(=\mathrm{O}) \mathrm{OC}) \mathrm{N}[\mathrm{C} @ @]([\mathrm{H}])(\mathrm{C} / \mathrm{C} 1=\mathrm{C} / \mathrm{C}=\mathrm{CC} \\
=\mathrm{C} 1)[\mathrm{C} @](\mathrm{O})([\mathrm{H}]) \mathrm{CN}(\mathrm{C}[\mathrm{C} @ @](\mathrm{C})([\mathrm{H}]) \mathrm{CC})[\mathrm{S}](=\mathrm{O})(=\mathrm{O}) \mathrm{C} 3=\mathrm{C} \\
\mathrm{C}=\mathrm{C} 2 \mathrm{~N}=\mathrm{C}[\mathrm{S}] \mathrm{C} 2=\mathrm{C} 3\end{array}$ \\
\hline $\mathrm{H} 27$ & \# & 3.400 & 4.170 & 71.848 & 4.116 & 89.365 & $\begin{array}{l}\mathrm{O}=\mathrm{C}(\mathrm{OCCN} 1 \mathrm{CCNC} 1=\mathrm{O}) \mathrm{N}[\mathrm{C} @ @]([\mathrm{H}])(\mathrm{C} / \mathrm{C} 2=\mathrm{C} / \mathrm{C}=\mathrm{CC}=\mathrm{C} 2)[\mathrm{C} \\
@](\mathrm{O})([\mathrm{H}]) \mathrm{CN}(\mathrm{CCCCC})[\mathrm{S}](=\mathrm{O})(=\mathrm{O}) \mathrm{C} 3=\mathrm{CC}=\mathrm{C}(\mathrm{C}=\mathrm{C} 3) \mathrm{OC}\end{array}$ \\
\hline H38 & \# & 4.770 & 5.203 & 89.640 & 5.300 & 115.081 & $\begin{array}{l}\mathrm{O}=\mathrm{C}(\mathrm{OCCN} 1 \mathrm{CCOC} 1=\mathrm{O}) \mathrm{N}[\mathrm{C} @ @]([\mathrm{H}])(\mathrm{C} / \mathrm{C} 2=\mathrm{C} / \mathrm{C}=\mathrm{CC}=\mathrm{C} 2)[\mathrm{C} \\
@](\mathrm{O})([\mathrm{H}]) \mathrm{CN}(\mathrm{CCCCC})[\mathrm{S}](=\mathrm{O})(=\mathrm{O}) \mathrm{C} 4=\mathrm{CC}=\mathrm{C} 3 \mathrm{~N}=\mathrm{C}[\mathrm{S}] \mathrm{C} 3=\mathrm{C} 4\end{array}$ \\
\hline H39 & $\#$ & 1.376 & 1.025 & 17.654 & 1.098 & 23.853 & $\begin{array}{l}\mathrm{O}=\mathrm{C}(\mathrm{O}[\mathrm{C} @] 1([\mathrm{H}]) \mathrm{CCOC} 1=\mathrm{O}) \mathrm{N}[\mathrm{C} @ @]([\mathrm{H}])(\mathrm{C} / \mathrm{C} 2=\mathrm{C} / \mathrm{C}=\mathrm{CC}=\mathrm{C} \\
\text { 2)[C@ }](\mathrm{O})([\mathrm{H}]) \mathrm{CN}(\mathrm{CCCCC})[\mathrm{S}](=\mathrm{O})(=\mathrm{O}) \mathrm{C} 4=\mathrm{CC}=\mathrm{C} 3 \mathrm{~N}=\mathrm{C}[\mathrm{S}] \mathrm{C} 3 \\
=\mathrm{C} 4\end{array}$ \\
\hline
\end{tabular}




\begin{tabular}{|c|c|c|c|c|c|c|c|}
\hline $\mathrm{H} 43$ & \# & 4.180 & 4.300 & 74.082 & 4.050 & 87.929 & $\begin{array}{l}\mathrm{O}=\mathrm{C} 1 \mathrm{O}[\mathrm{C} @ @]([\mathrm{H}])(\mathrm{CN} 1 \mathrm{C}=2 \mathrm{C}=\mathrm{CC}([\mathrm{F}])=\mathrm{C}([\mathrm{F}]) \mathrm{C}=2) \mathrm{C}(=\mathrm{O}) \mathrm{N}[\mathrm{C} \\
@ @]([\mathrm{H}])(\mathrm{C} / \mathrm{C} 3=\mathrm{C} / \mathrm{C}=\mathrm{CC}=\mathrm{C} 3)[\mathrm{C} @ @](\mathrm{O})([\mathrm{H}]) \mathrm{CN}(\mathrm{CC}(\mathrm{C})(\mathrm{C})[\mathrm{H} \\
])[\mathrm{S}](=\mathrm{O})(=\mathrm{O}) \mathrm{C} 4=\mathrm{CC}=\mathrm{C}(\mathrm{C}=\mathrm{C} 4) \mathrm{OC}\end{array}$ \\
\hline $\mathrm{H} 44$ & $\#$ & 5.222 & 3.892 & 67.051 & 4.061 & 88.169 & $\begin{array}{l}\mathrm{O}=\mathrm{C} 1 \mathrm{O}[\mathrm{C} @ @]([\mathrm{H}])(\mathrm{CN} 1 \mathrm{C}=2 \mathrm{C}=\mathrm{CC}=\mathrm{C}(\mathrm{C}=2) \mathrm{C}([\mathrm{F}])([\mathrm{F}])[\mathrm{F}]) \mathrm{C}(= \\
\mathrm{O}) \mathrm{N}[\mathrm{C} @ @]([\mathrm{H}])(\mathrm{C} / \mathrm{C} 3=\mathrm{C} / \mathrm{C}=\mathrm{CC}=\mathrm{C} 3)[\mathrm{C} @ @](\mathrm{O})([\mathrm{H}]) \mathrm{CN}(\mathrm{CC}(\mathrm{C} \\
)(\mathrm{C})[\mathrm{H}])[\mathrm{S}](=\mathrm{O})(=\mathrm{O}) \mathrm{C} 4=\mathrm{CC}=\mathrm{C}(\mathrm{C}=\mathrm{C} 4) \mathrm{OC}\end{array}$ \\
\hline H55 & $\#$ & 4.071 & 4.169 & 71.836 & 3.969 & 86.175 & $\begin{array}{l}\mathrm{O}=\mathrm{C} 1 \mathrm{O}[\mathrm{C} @ @]([\mathrm{H}])(\mathrm{CN} 1 \mathrm{C}=2 \mathrm{C}=\mathrm{CC}([\mathrm{F}])=\mathrm{C}([\mathrm{F}]) \mathrm{C}=2) \mathrm{C}(=\mathrm{O}) \mathrm{N}[\mathrm{C} \\
@ @ @([\mathrm{H}])(\mathrm{C} / \mathrm{C} 3=\mathrm{C} / \mathrm{C}=\mathrm{CC}=\mathrm{C} 3)[\mathrm{C} @ @](\mathrm{O})([\mathrm{H}]) \mathrm{CN}(\mathrm{CC}(\mathrm{C})(\mathrm{C})[\mathrm{H} \\
])[\mathrm{S}](=\mathrm{O})(=\mathrm{O}) \mathrm{C} 5=\mathrm{CC}=\mathrm{C} 4 \mathrm{OCOC} 4=\mathrm{C} 5\end{array}$ \\
\hline H58 & \# & 4.796 & 3.967 & 68.357 & 3.828 & 83.121 & $\begin{array}{l}\mathrm{O}=\mathrm{C} 1 \mathrm{O}[\mathrm{C} @ @]([\mathrm{H}])(\mathrm{CN} 1 \mathrm{C}=2 \mathrm{C}=\mathrm{CC}(=\mathrm{CC}=2) \mathrm{C}(=\mathrm{O}) \mathrm{C}) \mathrm{C}(=\mathrm{O}) \mathrm{N}[ \\
\mathrm{C} @ @]([\mathrm{H}])(\mathrm{C} / \mathrm{C} 3=\mathrm{C} / \mathrm{C}=\mathrm{CC}=\mathrm{C} 3)[\mathrm{C} @ @](\mathrm{O})([\mathrm{H}]) \mathrm{CN}(\mathrm{CC}(\mathrm{C})(\mathrm{C})[ \\
\mathrm{H}])[\mathrm{S}](=\mathrm{O})(=\mathrm{O}) \mathrm{C} 5=\mathrm{CC}=\mathrm{C} 4 \mathrm{OCOC} 4=\mathrm{C} 5\end{array}$ \\
\hline H75 & $\#$ & 0.795 & 0.701 & 12.077 & 0.250 & 5.444 & $\begin{array}{l}\mathrm{O}=\mathrm{C} 1 \mathrm{O}[\mathrm{C} @ @]([\mathrm{H}])(\mathrm{CN} 1 \mathrm{C}=2 \mathrm{C}=\mathrm{C} / \mathrm{C}=\mathrm{C}(/[\mathrm{F}]) \mathrm{C}=2) \mathrm{C}(=\mathrm{O}) \mathrm{N}[\mathrm{C} @ \\
@]([\mathrm{H}])(\mathrm{C} / \mathrm{C} 3=\mathrm{C} / \mathrm{C}=\mathrm{CC}=\mathrm{C} 3)[\mathrm{C} @ @](\mathrm{O})([\mathrm{H}]) \mathrm{CN}(\mathrm{C} / \mathrm{C} 4=\mathrm{C} / \mathrm{C}=\mathrm{C}[ \\
\mathrm{S}] 4)[\mathrm{S}](=\mathrm{O})(=\mathrm{O}) \mathrm{C} 5=\mathrm{CC}([\mathrm{F}])=\mathrm{C}([\mathrm{F}]) \mathrm{C}=\mathrm{C} 5[\mathrm{~F}]\end{array}$ \\
\hline H86 & $\#$ & 4.066 & 4.125 & 71.066 & 4.048 & 87.892 & $\begin{array}{l}\mathrm{O}=\mathrm{C} 1 \mathrm{O}[\mathrm{C} @ @]([\mathrm{H}])(\mathrm{CN} 1 \mathrm{C}=2 \mathrm{C}=\mathrm{CC}=\mathrm{CC}=2 \mathrm{C}([\mathrm{F}])([\mathrm{F}])[\mathrm{F}]) \mathrm{C}(=\mathrm{O} \\
) \mathrm{N}[\mathrm{C} @ @]([\mathrm{H}])(\mathrm{C} / \mathrm{C} 3=\mathrm{C} / \mathrm{C}=\mathrm{CC}=\mathrm{C} 3)[\mathrm{C} @ @](\mathrm{O})([\mathrm{H}]) \mathrm{CN}(\mathrm{CC}(\mathrm{C})( \\
\mathrm{C})[\mathrm{H}])[\mathrm{S}](=\mathrm{O})(=\mathrm{O}) \mathrm{C} 4=\mathrm{CC}=\mathrm{C}(\mathrm{C}=\mathrm{C} 4) \mathrm{OC}\end{array}$ \\
\hline $\mathrm{H} 90$ & \# & 3.415 & 3.342 & 57.579 & 3.370 & 73.171 & $\begin{array}{l}\mathrm{O}=\mathrm{C} 1 \mathrm{O}[\mathrm{C} @ @]([\mathrm{H}])(\mathrm{CN} 1 \mathrm{C}=2 \mathrm{C}=\mathrm{CC}([\mathrm{F}])=\mathrm{CC}=2[\mathrm{~F}]) \mathrm{C}(=\mathrm{O}) \mathrm{N}[\mathrm{C} \\
@ @]([\mathrm{H}])(\mathrm{C} / \mathrm{C} 3=\mathrm{C} / \mathrm{C}=\mathrm{CC}=\mathrm{C} 3)[\mathrm{C} @ @](\mathrm{O})([\mathrm{H}]) \mathrm{CN}(\mathrm{CC}(\mathrm{C})(\mathrm{C})[\mathrm{H} \\
])[\mathrm{S}](=\mathrm{O})(=\mathrm{O}) \mathrm{C} 4=\mathrm{C} / \mathrm{C}=\mathrm{C}(/ \mathrm{N}) \mathrm{C}=\mathrm{C} 4\end{array}$ \\
\hline H94 & $\#$ & 3.674 & 3.211 & 55.318 & 3.219 & 69.904 & $\begin{array}{l}\mathrm{O}=\mathrm{C} 1 \mathrm{O}[\mathrm{C} @ @]([\mathrm{H}])(\mathrm{CN} 1 \mathrm{C}=2 \mathrm{C}=\mathrm{CC}([\mathrm{F}])=\mathrm{CC}=2[\mathrm{~F}]) \mathrm{C}(=\mathrm{O}) \mathrm{N}[\mathrm{C} \\
@ @ @([\mathrm{H}])(\mathrm{C} / \mathrm{C} 3=\mathrm{C} / \mathrm{C}=\mathrm{CC}=\mathrm{C} 3)[\mathrm{C} @ @](\mathrm{O})([\mathrm{H}]) \mathrm{CN}(\mathrm{CC}(\mathrm{C})(\mathrm{C})[\mathrm{H} \\
])[\mathrm{S}](=\mathrm{O})(=\mathrm{O}) \mathrm{C} 4=\mathrm{CC}=\mathrm{C}(\mathrm{C}=\mathrm{C} 4) \mathrm{CO}\end{array}$ \\
\hline H99 & \# & 3.684 & 3.198 & 55.095 & 3.392 & 73.649 & $\begin{array}{l}\mathrm{O}=\mathrm{C} 1 \mathrm{O}[\mathrm{C} @ @]([\mathrm{H}])(\mathrm{CN} 1 \mathrm{C}=2 \mathrm{C}=\mathrm{CC}([\mathrm{F}])=\mathrm{CC}=2) \mathrm{C}(=\mathrm{O}) \mathrm{N}[\mathrm{C} @ @ \\
]([\mathrm{H}])(\mathrm{C} / \mathrm{C} 3=\mathrm{C} / \mathrm{C}=\mathrm{CC}=\mathrm{C} 3)[\mathrm{C} @ @](\mathrm{O})([\mathrm{H}]) \mathrm{CN}(\mathrm{CC}(\mathrm{C})(\mathrm{C})[\mathrm{H}])[\mathrm{S}] \\
(=\mathrm{O})(=\mathrm{O}) \mathrm{C} 4=\mathrm{CC}=\mathrm{C}(\mathrm{C}=\mathrm{C} 4) \mathrm{CO}\end{array}$ \\
\hline H103 & \# & 3.496 & 3.474 & 59.848 & 3.473 & 75.417 & $\begin{array}{l}\mathrm{O}=\mathrm{C} 1 \mathrm{O}[\mathrm{C} @ @]([\mathrm{H}])(\mathrm{CN} 1 \mathrm{C}=2 \mathrm{C}=\mathrm{C} / \mathrm{C}=\mathrm{C}(/[\mathrm{F}]) \mathrm{C}=2) \mathrm{C}(=\mathrm{O}) \mathrm{N}[\mathrm{C} @ \\
@]([\mathrm{H}])(\mathrm{C} / \mathrm{C} 3=\mathrm{C} / \mathrm{C}=\mathrm{CC}=\mathrm{C} 3)[\mathrm{C} @ @](\mathrm{O})([\mathrm{H}]) \mathrm{CN}(\mathrm{CC}(\mathrm{C})(\mathrm{C})[\mathrm{H}])[ \\
\mathrm{S}](=\mathrm{O})(=\mathrm{O}) \mathrm{C} 4=\mathrm{CC}=\mathrm{C}(\mathrm{C}=\mathrm{C} 4) \mathrm{CO}\end{array}$ \\
\hline H108 & $\#$ & 3.648 & 3.487 & 60.079 & 3.393 & 73.680 & $\begin{array}{l}\mathrm{O}=\mathrm{C} 1 \mathrm{O}[\mathrm{C} @ @]([\mathrm{H}])(\mathrm{CN} 1 \mathrm{C}=2 \mathrm{C}=\mathrm{CC}=\mathrm{C}(\mathrm{C}=2) \mathrm{OC}([\mathrm{FF}])([\mathrm{F}])[\mathrm{F}]) \mathrm{C}( \\
=\mathrm{O}) \mathrm{N}[\mathrm{C} @ @]([\mathrm{H}])(\mathrm{C} / \mathrm{C} 3=\mathrm{C} / \mathrm{C}=\mathrm{CC}=\mathrm{C} 3)[\mathrm{C} @ @](\mathrm{O})([\mathrm{H}]) \mathrm{CN}(\mathrm{CC}( \\
\mathrm{C})(\mathrm{C})[\mathrm{H}])[\mathrm{S}](=\mathrm{O})(=\mathrm{O}) \mathrm{C} 4=\mathrm{C} / \mathrm{C}=\mathrm{C}(/ \mathrm{N}) \mathrm{C}=\mathrm{C} 4\end{array}$ \\
\hline H112 & \# & 3.544 & 3.356 & 57.818 & 3.243 & 70.413 & $\begin{array}{l}\mathrm{O}=\mathrm{C} 1 \mathrm{O}[\mathrm{C} @ @]([\mathrm{H}])(\mathrm{CN} 1 \mathrm{C}=2 \mathrm{C}=\mathrm{CC}=\mathrm{C}(\mathrm{C}=2) \mathrm{OC}([\mathrm{F}])([\mathrm{F}])[\mathrm{F}]) \mathrm{C}( \\
=\mathrm{O}) \mathrm{N}[\mathrm{C} @ @]([\mathrm{H}])(\mathrm{C} / \mathrm{C} 3=\mathrm{C} / \mathrm{C}=\mathrm{CC}=\mathrm{C} 3)[\mathrm{C} @ @](\mathrm{O})([\mathrm{H}]) \mathrm{CN}(\mathrm{CC}( \\
\mathrm{C})(\mathrm{C})[\mathrm{H}])[\mathrm{S}](=\mathrm{O})(=\mathrm{O}) \mathrm{C} 4=\mathrm{CC}=\mathrm{C}(\mathrm{C}=\mathrm{C} 4) \mathrm{CO}\end{array}$ \\
\hline H122 & \# & 3.947 & 4.666 & 80.388 & 4.670 & 101.386 & $\begin{array}{l}\mathrm{O}=\mathrm{C} 1 \mathrm{O}[\mathrm{C} @ @]([\mathrm{H}])(\mathrm{CN} 1 \mathrm{C}=2 \mathrm{C}=\mathrm{C} / \mathrm{C}=\mathrm{C}(/ \mathrm{N}) \mathrm{C}=2) \mathrm{C}(=\mathrm{O}) \mathrm{N}[\mathrm{C} @ @ \\
]([\mathrm{H}])(\mathrm{C} / \mathrm{C} 3=\mathrm{C} / \mathrm{C}=\mathrm{CC}=\mathrm{C} 3)[\mathrm{C} @ @](\mathrm{O})([\mathrm{H}]) \mathrm{CN}(\mathrm{CC}(\mathrm{C})(\mathrm{C})[\mathrm{H}])[\mathrm{S}] \\
(=\mathrm{O})(=\mathrm{O}) \mathrm{C} 5=\mathrm{CC}=\mathrm{C} 4 \mathrm{OCOC} 4=\mathrm{C} 5\end{array}$ \\
\hline H126 & \# & 5.222 & 4.315 & 74.346 & 4.371 & 94.909 & $\begin{array}{l}\mathrm{O}=\mathrm{C} 1 \mathrm{O}[\mathrm{C} @ @]([\mathrm{H}])(\mathrm{CN} 1 \mathrm{C}=2 \mathrm{C}=\mathrm{CC}=\mathrm{C}(\mathrm{C}=2) \mathrm{NC}(=\mathrm{O}) \mathrm{C}) \mathrm{C}(=\mathrm{O}) \mathrm{N} \\
{[\mathrm{C} @ @]([\mathrm{H}])(\mathrm{C} / \mathrm{C} 3=\mathrm{C} / \mathrm{C}=\mathrm{CC}=\mathrm{C} 3)[\mathrm{C} @ @](\mathrm{O})([\mathrm{H}]) \mathrm{CN}(\mathrm{CC}(\mathrm{C})(\mathrm{C})[} \\
\mathrm{H}])[\mathrm{S}](=\mathrm{O})(=\mathrm{O}) \mathrm{C} 5=\mathrm{CC}=\mathrm{C} 4 \mathrm{~N}=\mathrm{C}[\mathrm{S}] \mathrm{C} 4=\mathrm{C} 5\end{array}$ \\
\hline $\mathrm{H} 4$ & $*$ & 5.523 & 5.315 & 91.576 & 5.383 & 116.866 & $\begin{array}{l}\mathrm{O}=\mathrm{C}(\mathrm{OCCNC}(=\mathrm{O}) \mathrm{C}([\mathrm{Cl}])([\mathrm{Cl}])[\mathrm{H}]) \mathrm{N}[\mathrm{C} @ @]([\mathrm{H}])(\mathrm{C} / \mathrm{C} 1=\mathrm{C} / \mathrm{C}=\mathrm{C} \\
\mathrm{C}=\mathrm{C} 1)[\mathrm{C} @(\mathrm{O})([\mathrm{H}]) \mathrm{CN}(\mathrm{C}[\mathrm{C} @ @](\mathrm{C})([\mathrm{H}]) \mathrm{CC})[\mathrm{S}](=\mathrm{O})(=\mathrm{O}) \mathrm{C} 2= \\
\mathrm{CC}=\mathrm{C}(\mathrm{C}=\mathrm{C} 2) \mathrm{OC}\end{array}$ \\
\hline H11 & $*$ & 4.260 & 4.912 & 84.626 & 6.351 & 137.880 & $\begin{array}{l}\mathrm{O}=\mathrm{C}(\mathrm{OC}[\mathrm{C} @ @] 1([\mathrm{H}]) \mathrm{NOCC} 1) \mathrm{N}[\mathrm{C} @ @]([\mathrm{H}])(\mathrm{C} / \mathrm{C} 2=\mathrm{C} / \mathrm{C}=\mathrm{CC}= \\
\mathrm{C} 2)[\mathrm{C} @](\mathrm{O})([\mathrm{H}]) \mathrm{CN}(\mathrm{C}[\mathrm{C} @ @](\mathrm{C})([\mathrm{H}]) \mathrm{CC})[\mathrm{S}](=\mathrm{O})(=\mathrm{O}) \mathrm{C} 3=\mathrm{CC} \\
=\mathrm{C}(\mathrm{C}=\mathrm{C} 3) \mathrm{OC}\end{array}$ \\
\hline H15 & $*$ & 3.519 & 3.199 & 55.113 & 3.255 & 70.676 & $\mathrm{O}=\mathrm{C}(\mathrm{O}[\mathrm{C} @ @](\mathrm{C})([\mathrm{H}]) \mathrm{CCC}(\mathrm{C})=\mathrm{O}) \mathrm{N}[\mathrm{C} @ @]([\mathrm{H}])(\mathrm{C} / \mathrm{C} 1=\mathrm{C} / \mathrm{C}=\mathrm{C}$ \\
\hline
\end{tabular}




\begin{tabular}{|c|c|c|c|c|c|c|c|}
\hline & & & & & & & $\begin{array}{l}\mathrm{C}=\mathrm{C} 1)[\mathrm{C} @](\mathrm{O})([\mathrm{H}]) \mathrm{CN}(\mathrm{C}[\mathrm{C} @ @](\mathrm{C})([\mathrm{H}]) \mathrm{CC})[\mathrm{S}](=\mathrm{O})(=\mathrm{O}) \mathrm{C} 3= \\
\mathrm{CC}=\mathrm{C} 2 \mathrm{~N}=\mathrm{C}[\mathrm{S}] \mathrm{C} 2=\mathrm{C} 3\end{array}$ \\
\hline H19 & $*$ & 5.301 & 5.768 & 99.388 & 5.877 & 127.601 & $\begin{array}{l}\mathrm{O}=\mathrm{C}(\mathrm{OCCN} 1 \mathrm{CCOC} 1=\mathrm{O}) \mathrm{N}[\mathrm{C} @ @]([\mathrm{H}])(\mathrm{C} / \mathrm{C} 2=\mathrm{C} / \mathrm{C}=\mathrm{CC}=\mathrm{C} 2)[\mathrm{C} \\
@](\mathrm{O})([\mathrm{H}]) \mathrm{CN}(\mathrm{C}[\mathrm{C} @ @](\mathrm{C})([\mathrm{H}]) \mathrm{CC})[\mathrm{S}](=\mathrm{O})(=\mathrm{O}) \mathrm{C} 4=\mathrm{CC}=\mathrm{C} 3 \mathrm{~N} \\
=\mathrm{C}[\mathrm{S}] \mathrm{C} 3=\mathrm{C} 4\end{array}$ \\
\hline $\mathrm{H} 23$ & $*$ & 1.503 & 1.381 & 23.788 & 1.307 & 28.404 & $\begin{array}{l}\mathrm{O}=\mathrm{C}(\mathrm{OCCNC}(=\mathrm{O}) \mathrm{C}([\mathrm{F}])([\mathrm{F}])[\mathrm{F}]) \mathrm{N}[\mathrm{C} @ @]([\mathrm{H}])(\mathrm{C} / \mathrm{C} 1=\mathrm{C} / \mathrm{C}=\mathrm{CC} \\
=\mathrm{C} 1)[\mathrm{C} @](\mathrm{O})([\mathrm{H}]) \mathrm{CN}(\mathrm{CCCCC})[\mathrm{S}](=\mathrm{O})(=\mathrm{O}) \mathrm{C} 2=\mathrm{CC}=\mathrm{C}(\mathrm{C}=\mathrm{C} 2) \mathrm{O} \\
\mathrm{C}\end{array}$ \\
\hline H31 & $*$ & 1.943 & 2.228 & 38.382 & 2.137 & 46.409 & $\begin{array}{l}\mathrm{O}=\mathrm{C}(\mathrm{O}[\mathrm{C} @ @](\mathrm{C})([\mathrm{H}]) \mathrm{CC}(=\mathrm{O}) \mathrm{OC}) \mathrm{N}[\mathrm{C} @ @]([\mathrm{H}])(\mathrm{C} / \mathrm{C} 1=\mathrm{C} / \mathrm{C}=\mathrm{C} \\
\mathrm{C}=\mathrm{C} 1)[\mathrm{C} @](\mathrm{O})([\mathrm{H}]) \mathrm{CN}(\mathrm{CCCC})[\mathrm{S}](=\mathrm{O})(=\mathrm{O}) \mathrm{C} 3=\mathrm{CC}=\mathrm{C} 2 \mathrm{~N}=\mathrm{C}[ \\
\mathrm{S}] \mathrm{C} 2=\mathrm{C} 3\end{array}$ \\
\hline H32 & $*$ & 1.706 & 1.453 & 25.037 & 1.365 & 29.656 & $\begin{array}{l}\mathrm{O}=\mathrm{C}(\mathrm{OCCNC}(=\mathrm{O}) \mathrm{C}([\mathrm{F}])([\mathrm{F}])[\mathrm{F}]) \mathrm{N}[\mathrm{C} @ @]([\mathrm{H}])(\mathrm{C} / \mathrm{C} 1=\mathrm{C} / \mathrm{C}=\mathrm{CC} \\
=\mathrm{C} 1)[\mathrm{C} @](\mathrm{O})([\mathrm{H}]) \mathrm{CN}(\mathrm{CCCCC})[\mathrm{S}](=\mathrm{O})(=\mathrm{O}) \mathrm{C} 3=\mathrm{CC}=\mathrm{C} 2 \mathrm{~N}=\mathrm{C}[\mathrm{S}] \\
\mathrm{C} 2=\mathrm{C} 3\end{array}$ \\
\hline H37 & $*$ & 3.664 & 4.389 & 75.614 & 4.380 & 95.105 & $\begin{array}{l}\mathrm{O}=\mathrm{C}(\mathrm{OCCN} 1 \mathrm{CCNC} 1=\mathrm{O}) \mathrm{N}[\mathrm{C} @ @]([\mathrm{H}])(\mathrm{C} / \mathrm{C} 2=\mathrm{C} / \mathrm{C}=\mathrm{CC}=\mathrm{C} 2)[\mathrm{C} \\
@](\mathrm{O})([\mathrm{H}]) \mathrm{CN}(\mathrm{CCCCC})[\mathrm{S}](=\mathrm{O})(=\mathrm{O}) \mathrm{C} 4=\mathrm{CC}=\mathrm{C} 3 \mathrm{~N}=\mathrm{C}[\mathrm{S}] \mathrm{C} 3=\mathrm{C} 4\end{array}$ \\
\hline $\mathrm{H} 41$ & $*$ & 4.000 & 3.881 & 66.867 & 3.770 & 81.867 & $\begin{array}{l}\mathrm{O}=\mathrm{C} 1 \mathrm{O}[\mathrm{C} @ @]([\mathrm{H}])(\mathrm{CN} 1 \mathrm{C}=2 \mathrm{C}=\mathrm{CC}=\mathrm{CC}=2) \mathrm{C}(=\mathrm{O}) \mathrm{N}[\mathrm{C} @ @]([\mathrm{H}] \\
)(\mathrm{C} / \mathrm{C} 3=\mathrm{C} / \mathrm{C}=\mathrm{CC}=\mathrm{C} 3)[\mathrm{C} @ @](\mathrm{O})([\mathrm{H}]) \mathrm{CN}(\mathrm{CC}(\mathrm{C})(\mathrm{C})[\mathrm{H}])[\mathrm{S}](=\mathrm{O})( \\
=\mathrm{O}) \mathrm{C} 4=\mathrm{CC}=\mathrm{C}(\mathrm{C}=\mathrm{C} 4) \mathrm{OC}\end{array}$ \\
\hline H46 & $*$ & 5.398 & 4.098 & 70.603 & 3.909 & 84.874 & $\begin{array}{l}\mathrm{O}=\mathrm{C} 1 \mathrm{O}[\mathrm{C} @ @]([\mathrm{H}])(\mathrm{CN} 1 \mathrm{C}=2 \mathrm{C}=\mathrm{CC}(=\mathrm{CC}=2) \mathrm{C}(\mathrm{C})=\mathrm{O}) \mathrm{C}(=\mathrm{O}) \mathrm{N}[ \\
\mathrm{C} @ @]([\mathrm{H}])(\mathrm{C} / \mathrm{C} 3=\mathrm{C} / \mathrm{C}=\mathrm{CC}=\mathrm{C} 3)[\mathrm{C} @ @](\mathrm{O})([\mathrm{H}]) \mathrm{CN}(\mathrm{CC}(\mathrm{C})(\mathrm{C})[ \\
\mathrm{H}])[\mathrm{S}](=\mathrm{O})(=\mathrm{O}) \mathrm{C} 4=\mathrm{CC}=\mathrm{C}(\mathrm{C}=\mathrm{C} 4) \mathrm{OC}\end{array}$ \\
\hline H56 & $*$ & 4.796 & 3.761 & 64.805 & 3.980 & 86.416 & $\begin{array}{l}\mathrm{O}=\mathrm{C} 1 \mathrm{O}[\mathrm{C} @ @]([\mathrm{H}])(\mathrm{CN} 1 \mathrm{C}=2 \mathrm{C}=\mathrm{CC}=\mathrm{C}(\mathrm{C}=2) \mathrm{C}([\mathrm{F}])([\mathrm{F}])[\mathrm{F}]) \mathrm{C}(= \\
\mathrm{O}) \mathrm{N}[\mathrm{C} @ @]([\mathrm{H}])(\mathrm{C} / \mathrm{C} 3=\mathrm{C} / \mathrm{C}=\mathrm{CC}=\mathrm{C} 3)[\mathrm{C} @ @](\mathrm{O})([\mathrm{H}]) \mathrm{CN}(\mathrm{CC}(\mathrm{C} \\
)(\mathrm{C})[\mathrm{H}])[\mathrm{S}](=\mathrm{O})(=\mathrm{O}) \mathrm{C} 5=\mathrm{CC}=\mathrm{C} 4 \mathrm{OCOC} 4=\mathrm{C} 5\end{array}$ \\
\hline H60 & $*$ & 3.465 & 4.286 & 73.837 & 4.085 & 88.700 & $\begin{array}{l}\mathrm{O}=\mathrm{C} 1 \mathrm{O}[\mathrm{C} @ @]([\mathrm{H}])(\mathrm{CN} 1 \mathrm{C}=2 \mathrm{C}=\mathrm{CC}([\mathrm{F}])=\mathrm{C}([\mathrm{F}]) \mathrm{C}=2) \mathrm{C}(=\mathrm{O}) \mathrm{N}[\mathrm{C} \\
@ @]([\mathrm{H}])(\mathrm{C} / \mathrm{C} 3=\mathrm{C} / \mathrm{C}=\mathrm{CC}=\mathrm{C} 3)[\mathrm{C} @ @](\mathrm{O})([\mathrm{H}]) \mathrm{CN}(\mathrm{CC}(\mathrm{C})(\mathrm{C})[\mathrm{H} \\
])[\mathrm{S}](=\mathrm{O})(=\mathrm{O}) \mathrm{C} 4=\mathrm{C} / \mathrm{C}=\mathrm{C}(/ \mathrm{OC}) \mathrm{C}(\mathrm{C})=\mathrm{C} 4\end{array}$ \\
\hline $\mathrm{H} 70$ & $*$ & 3.097 & 3.503 & 60.362 & 3.493 & 75.851 & $\begin{array}{l}\mathrm{O}=\mathrm{C} 1 \mathrm{O}[\mathrm{C} @ @]([\mathrm{H}])(\mathrm{CN} 1 \mathrm{C}=2 \mathrm{C}=\mathrm{CC}(=\mathrm{CC}=2) \mathrm{C}(\mathrm{C})=\mathrm{O}) \mathrm{C}(=\mathrm{O}) \mathrm{N}[ \\
\mathrm{C} @ @]([\mathrm{H}])(\mathrm{C} / \mathrm{C} 3=\mathrm{C} / \mathrm{C}=\mathrm{CC}=\mathrm{C} 3)[\mathrm{C} @ @](\mathrm{O})([\mathrm{H}]) \mathrm{CN}(\mathrm{C}[\mathrm{C} @ @] 4( \\
[\mathrm{H}]) \mathrm{CC} 4)[\mathrm{S}](=\mathrm{O})(=\mathrm{O}) \mathrm{C} 5=\mathrm{CC}=\mathrm{C}(\mathrm{C}=\mathrm{C} 5) \mathrm{OC}\end{array}$ \\
\hline H76 & $*$ & 0.775 & 0.962 & 16.575 & 0.928 & 20.162 & $\begin{array}{l}\mathrm{O}=\mathrm{C} 1 \mathrm{O}[\mathrm{C} @ @]([\mathrm{H}])(\mathrm{CN} 1 \mathrm{C}=2 \mathrm{C}=\mathrm{CC}(=\mathrm{CC}=2) \mathrm{C}(=\mathrm{O}) \mathrm{C}) \mathrm{C}(=\mathrm{O}) \mathrm{N}[ \\
\mathrm{C} @ @]([\mathrm{H}])(\mathrm{C} / \mathrm{C} 3=\mathrm{C} / \mathrm{C}=\mathrm{CC}=\mathrm{C} 3)[\mathrm{C} @ @](\mathrm{O})([\mathrm{H}]) \mathrm{CN}(\mathrm{C} / \mathrm{C} 4=\mathrm{C} / \mathrm{C} \\
=\mathrm{C}[\mathrm{S}] 4)[\mathrm{S}](=\mathrm{O})(=\mathrm{O}) \mathrm{C} 5=\mathrm{CC}([\mathrm{F}])=\mathrm{C}([\mathrm{F}]) \mathrm{C}=\mathrm{C} 5[\mathrm{~F}]\end{array}$ \\
\hline H80 & $*$ & 4.481 & 4.127 & 71.109 & 4.105 & 89.132 & $\begin{array}{l}\mathrm{O}=\mathrm{C} 1 \mathrm{O}[\mathrm{C} @ @]([\mathrm{H}])(\mathrm{CN} 1 \mathrm{C}=2 \mathrm{C}=\mathrm{CC}=\mathrm{CC}=2) \mathrm{C}(=\mathrm{O}) \mathrm{N}[\mathrm{C} @ @]([\mathrm{H}] \\
)(\mathrm{C} / \mathrm{C} 3=\mathrm{C} / \mathrm{C}=\mathrm{CC}=\mathrm{C} 3)[\mathrm{C} @ @](\mathrm{O})([\mathrm{H}]) \mathrm{CN}(\mathrm{CC}(\mathrm{C})(\mathrm{C})[\mathrm{H}])[\mathrm{S}](=\mathrm{O})( \\
=\mathrm{O}) \mathrm{C} 5=\mathrm{CC}=\mathrm{C} 4 \mathrm{~N}=\mathrm{C}[\mathrm{S}] \mathrm{C} 4=\mathrm{C} 5\end{array}$ \\
\hline H84 & $*$ & 2.833 & 2.386 & 41.107 & 2.422 & 52.603 & $\begin{array}{l}\mathrm{O}=\mathrm{C} 1 \mathrm{O}[\mathrm{C} @ @]([\mathrm{H}])(\mathrm{CN} 1 \mathrm{C}=2 \mathrm{C}=\mathrm{CC}=\mathrm{CC}=2 \mathrm{O}) \mathrm{C}(=\mathrm{O}) \mathrm{N}[\mathrm{C} @ @]([ \\
\mathrm{H}])(\mathrm{C} / \mathrm{C} 3=\mathrm{C} / \mathrm{C}=\mathrm{CC}=\mathrm{C} 3)[\mathrm{C} @ @](\mathrm{O})([\mathrm{H}]) \mathrm{CN}(\mathrm{CC}(\mathrm{C})(\mathrm{C})[\mathrm{H}])[\mathrm{S}](= \\
\mathrm{O})(=\mathrm{O}) \mathrm{C} 5=\mathrm{CC}=\mathrm{C} 4 \mathrm{OCOC} 4=\mathrm{C} 5\end{array}$ \\
\hline H117 & $*$ & 5.523 & 4.764 & 82.082 & 4.890 & 106.165 & $\begin{array}{l}\mathrm{O}=\mathrm{C} 1 \mathrm{O}[\mathrm{C} @ @]([\mathrm{H}])(\mathrm{CN} 1 \mathrm{C}=2 \mathrm{C}=\mathrm{CC}=\mathrm{C}(\mathrm{C}=2)[\mathrm{S}](\mathrm{C})(=\mathrm{O})=\mathrm{O}) \mathrm{C}(= \\
\mathrm{O}) \mathrm{N}[\mathrm{C} @ @]([\mathrm{H}])(\mathrm{C} / \mathrm{C} 3=\mathrm{C} / \mathrm{C}=\mathrm{CC}=\mathrm{C} 3)[\mathrm{C} @ @](\mathrm{O})([\mathrm{H}]) \mathrm{CN}(\mathrm{CC}(\mathrm{C} \\
)(\mathrm{C})[\mathrm{H}])[\mathrm{S}](=\mathrm{O})(=\mathrm{O}) \mathrm{C} 5=\mathrm{CC}=\mathrm{C} 4 \mathrm{~N}=\mathrm{C}[\mathrm{S}] \mathrm{C} 4=\mathrm{C} 5\end{array}$ \\
\hline H125 & $*$ & 4.292 & 3.938 & 67.858 & 3.956 & 85.890 & $\begin{array}{l}\mathrm{O}=\mathrm{C} 1 \mathrm{O}[\mathrm{C} @ @]([\mathrm{H}])(\mathrm{CN} 1 \mathrm{C}=2 \mathrm{C}=\mathrm{CC}=\mathrm{C}(\mathrm{C}=2) \mathrm{NC}(=\mathrm{O}) \mathrm{C}) \mathrm{C}(=\mathrm{O}) \mathrm{N} \\
{[\mathrm{C} @ @]([\mathrm{H}])(\mathrm{C} / \mathrm{C} 3=\mathrm{C} / \mathrm{C}=\mathrm{CC}=\mathrm{C} 3)[\mathrm{C} @ @](\mathrm{O})([\mathrm{H}]) \mathrm{CN}(\mathrm{CC}(\mathrm{C})(\mathrm{C})[} \\
\mathrm{H}])[\mathrm{S}](=\mathrm{O})(=\mathrm{O}) \mathrm{C} 5=\mathrm{CC}=\mathrm{C} 4 \mathrm{OCOC} 4=\mathrm{C} 5\end{array}$ \\
\hline
\end{tabular}

${ }^{1}$ Experimental $p K_{I},{ }^{2}$ Calculated $p K_{i}$ 
Table S2: Observed, predicted activity and DCW, and distribution into the training (+), calibration (-), test (\#) and validation (*) sets of split 2 (Models 3 and 4)

\begin{tabular}{|c|c|c|c|c|c|c|c|}
\hline \multirow{2}{*}{$\begin{array}{l}\text { Mol. } \\
\text { No. }\end{array}$} & \multirow{2}{*}{ Set } & \multirow{2}{*}{${ }^{1}$ Exp. } & \multicolumn{2}{|c|}{ Model 3} & \multicolumn{2}{|c|}{ Model 4} & \multirow{2}{*}{ SMILES } \\
\hline & & & ${ }^{2}$ Calc. & DCW & ${ }^{2}$ Calc. & DCW & \\
\hline H1 & + & 3.614 & 3.867 & 79.727 & 3.584 & 54.876 & $\begin{array}{l}\mathrm{O}=\mathrm{C}(\mathrm{OC}(\mathrm{C})(\mathrm{C}) \mathrm{C}) \mathrm{N}[\mathrm{C} @ @]([\mathrm{H}])(\mathrm{C} / \mathrm{C} 1=\mathrm{C} / \mathrm{C}=\mathrm{CC}=\mathrm{C} 1)[\mathrm{C} @](\mathrm{O})([\mathrm{H}]) \mathrm{C} \\
\mathrm{N}(\mathrm{C}[\mathrm{C} @ @](\mathrm{C})([\mathrm{H}]) \mathrm{CC})[\mathrm{S}](=\mathrm{O})(=\mathrm{O}) \mathrm{C} 3=\mathrm{CC}=\mathrm{C} 2 \mathrm{~N}=\mathrm{C}[\mathrm{S}] \mathrm{C} 2=\mathrm{C} 3\end{array}$ \\
\hline $\mathrm{H} 4$ & + & 5.523 & 5.144 & 106.029 & 5.097 & 78.081 & $\begin{array}{l}\mathrm{O}=\mathrm{C}(\mathrm{OCCNC}(=\mathrm{O}) \mathrm{C}([\mathrm{Cl}])([\mathrm{Cl}])[\mathrm{H}]) \mathrm{N}[\mathrm{C} @ @]([\mathrm{H}])(\mathrm{C} / \mathrm{C} 1=\mathrm{C} / \mathrm{C}=\mathrm{CC}=\mathrm{C} 1 \\
)[\mathrm{C} @](\mathrm{O})([\mathrm{H}]) \mathrm{CN}(\mathrm{C}[\mathrm{C} @ @](\mathrm{C})([\mathrm{H}]) \mathrm{CC})[\mathrm{S}](=\mathrm{O})(=\mathrm{O}) \mathrm{C} 2=\mathrm{CC}=\mathrm{C}(\mathrm{C}=\mathrm{C} 2 \\
\mathrm{OOC}\end{array}$ \\
\hline H6 & + & 2.697 & 3.040 & 62.686 & 2.978 & 45.592 & $\begin{array}{l}\mathrm{O}=\mathrm{C}(\mathrm{OCCNC}(=\mathrm{O}) \mathrm{OC}(\mathrm{C})(\mathrm{C}) \mathrm{C}) \mathrm{N}[\mathrm{C} @ @]([\mathrm{H}])(\mathrm{C} / \mathrm{C} 1=\mathrm{C} / \mathrm{C}=\mathrm{CC}=\mathrm{C} 1)[\mathrm{C} \\
@](\mathrm{O})([\mathrm{H}]) \mathrm{CN}(\mathrm{C}[\mathrm{C} @ @](\mathrm{C})([\mathrm{H}]) \mathrm{CC})[\mathrm{S}](=\mathrm{O})(=\mathrm{O}) \mathrm{C} 2=\mathrm{CC}=\mathrm{C}(\mathrm{C}=\mathrm{C} 2) \mathrm{O} \\
\mathrm{C}\end{array}$ \\
\hline $\mathrm{H} 8$ & + & 4.319 & 4.148 & 85.512 & 4.138 & 63.375 & $\begin{array}{l}\mathrm{O}=\mathrm{C}(\mathrm{OCCN} 1 \mathrm{CCNC} 1=\mathrm{O}) \mathrm{N}[\mathrm{C} @ @]([\mathrm{H}])(\mathrm{C} / \mathrm{C} 2=\mathrm{C} / \mathrm{C}=\mathrm{CC}=\mathrm{C} 2)[\mathrm{C} @](\mathrm{O}) \\
([\mathrm{H}]) \mathrm{CN}(\mathrm{C}[\mathrm{C} @ @](\mathrm{C})([\mathrm{H}]) \mathrm{CC})[\mathrm{S}](=\mathrm{O})(=\mathrm{O}) \mathrm{C} 3=\mathrm{CC}=\mathrm{C}(\mathrm{C}=\mathrm{C} 3) \mathrm{OC}\end{array}$ \\
\hline H9 & + & 5.523 & 5.447 & 112.269 & 5.377 & 82.376 & $\begin{array}{l}\mathrm{O}=\mathrm{C}(\mathrm{OCCN} 1 \mathrm{CCOC} 1=\mathrm{O}) \mathrm{N}[\mathrm{C} @ @]([\mathrm{H}])(\mathrm{C} / \mathrm{C} 2=\mathrm{C} / \mathrm{C}=\mathrm{CC}=\mathrm{C} 2)[\mathrm{C} @](\mathrm{O}) \\
([\mathrm{H}]) \mathrm{CN}(\mathrm{C}[\mathrm{C} @ @](\mathrm{C})([\mathrm{H}]) \mathrm{CC})[\mathrm{S}](=\mathrm{O})(=\mathrm{O}) \mathrm{C} 3=\mathrm{CC}=\mathrm{C}(\mathrm{C}=\mathrm{C} 3) \mathrm{OC}\end{array}$ \\
\hline H11 & + & 4.260 & 4.245 & 87.510 & 4.250 & 65.087 & $\begin{array}{l}\mathrm{O}=\mathrm{C}(\mathrm{OC}[\mathrm{C} @ @] 1([\mathrm{H}]) \mathrm{NOCC} 1) \mathrm{N}[\mathrm{C} @ @]([\mathrm{H}])(\mathrm{C} / \mathrm{C} 2=\mathrm{C} / \mathrm{C}=\mathrm{CC}=\mathrm{C} 2)[\mathrm{C} \\
@](\mathrm{O})([\mathrm{H}]) \mathrm{CN}(\mathrm{C}[\mathrm{C} @ @](\mathrm{C})([\mathrm{H}]) \mathrm{CC})[\mathrm{S}](=\mathrm{O})(=\mathrm{O}) \mathrm{C} 3=\mathrm{CC}=\mathrm{C}(\mathrm{C}=\mathrm{C} 3) \mathrm{O} \\
\mathrm{C}\end{array}$ \\
\hline H13 & + & 2.040 & 2.364 & 48.765 & 2.378 & 36.385 & $\begin{array}{l}\mathrm{O}=\mathrm{C}(\mathrm{OCCNC}(=\mathrm{O}) \mathrm{C}([\mathrm{F}])([\mathrm{F}])[\mathrm{F}]) \mathrm{N}[\mathrm{C} @ @]((\mathrm{H}])(\mathrm{C} / \mathrm{C} 1=\mathrm{C} / \mathrm{C}=\mathrm{CC}=\mathrm{C} 1)[ \\
\mathrm{C} @](\mathrm{O})([\mathrm{H}]) \mathrm{CN}(\mathrm{C}[\mathrm{C} @ @](\mathrm{C})([\mathrm{H}]) \mathrm{CC})[\mathrm{S}](=\mathrm{O})(=\mathrm{O}) \mathrm{C} 3=\mathrm{CC}=\mathrm{C} 2 \mathrm{~N}=\mathrm{C}[\mathrm{S} \\
] \mathrm{C} 2=\mathrm{C} 3\end{array}$ \\
\hline H14 & + & 4.222 & 5.545 & 114.300 & 5.522 & 84.606 & $\begin{array}{l}\mathrm{O}=\mathrm{C}(\mathrm{OCCNC}(=\mathrm{O}) \mathrm{C}([\mathrm{Cl}])([\mathrm{Cl}])[\mathrm{H}]) \mathrm{N}[\mathrm{C} @ @]([\mathrm{H}])(\mathrm{C} / \mathrm{C} 1=\mathrm{C} / \mathrm{C}=\mathrm{CC}=\mathrm{C} 1 \\
)[\mathrm{C} @](\mathrm{O})([\mathrm{H}]) \mathrm{CN}(\mathrm{C}[\mathrm{C} @ @](\mathrm{C})([\mathrm{H}]) \mathrm{CC})[\mathrm{S}](=\mathrm{O})(=\mathrm{O}) \mathrm{C} 3=\mathrm{CC}=\mathrm{C} 2 \mathrm{~N}=\mathrm{Cl} \\
\mathrm{S}] \mathrm{C} 2=\mathrm{C} 3\end{array}$ \\
\hline H16 & + & 3.638 & 3.441 & 70.957 & 3.404 & 52.117 & $\begin{array}{l}\mathrm{O}=\mathrm{C}(\mathrm{OCCNC}(=\mathrm{O}) \mathrm{OC}(\mathrm{C})(\mathrm{C}) \mathrm{C}) \mathrm{N}[\mathrm{C} @ @]([\mathrm{H}])(\mathrm{C} / \mathrm{C} 1=\mathrm{C} / \mathrm{C}=\mathrm{CC}=\mathrm{C} 1)[\mathrm{C} \\
@](\mathrm{O})([\mathrm{H}]) \mathrm{CN}(\mathrm{C}[\mathrm{C} @ @](\mathrm{C})([\mathrm{H}]) \mathrm{CC})[\mathrm{S}](=\mathrm{O})(=\mathrm{O}) \mathrm{C} 3=\mathrm{CC}=\mathrm{C} 2 \mathrm{~N}=\mathrm{C}[\mathrm{S}] \\
\mathrm{C} 2=\mathrm{C} 3\end{array}$ \\
\hline H19 & + & 5.301 & 5.519 & 113.764 & 5.460 & 83.650 & $\begin{array}{l}\mathrm{O}=\mathrm{C}(\mathrm{OCCN} 1 \mathrm{CCOC} 1=\mathrm{O}) \mathrm{N}[\mathrm{C} @ @]([\mathrm{H}])(\mathrm{C} / \mathrm{C2}=\mathrm{C} / \mathrm{C}=\mathrm{CC}=\mathrm{C} 2)[\mathrm{C} @](\mathrm{O}) \\
([\mathrm{H}]) \mathrm{CN}(\mathrm{C}[\mathrm{C} @ @](\mathrm{C})([\mathrm{H}]) \mathrm{CC})[\mathrm{S}](=\mathrm{O})(=\mathrm{O}) \mathrm{C4}=\mathrm{CC}=\mathrm{C} 3 \mathrm{~N}=\mathrm{C}[\mathrm{S}] \mathrm{C} 3=\mathrm{C4}\end{array}$ \\
\hline $\mathrm{H} 21$ & + & 5.523 & 4.728 & 97.476 & 4.659 & 71.365 & $\begin{array}{l}\mathrm{O}=\mathrm{C}(\mathrm{OC}[\mathrm{C} @] 1([\mathrm{H}]) \mathrm{NC}(=\mathrm{O}) \mathrm{CC} 1) \mathrm{N}[\mathrm{C} @ @]([\mathrm{H}])(\mathrm{C} / \mathrm{C} 2=\mathrm{C} / \mathrm{C}=\mathrm{CC}=\mathrm{C} 2) \\
{[\mathrm{C} @](\mathrm{O})([\mathrm{H}]) \mathrm{CN}(\mathrm{C}[\mathrm{C} @ @](\mathrm{C})([\mathrm{H}]) \mathrm{CC})[\mathrm{S}](=\mathrm{O})(=\mathrm{O}) \mathrm{C} 4=\mathrm{CC}=\mathrm{C} 3 \mathrm{~N}=\mathrm{C}[} \\
\mathrm{S}] \mathrm{C} 3=\mathrm{C} 4\end{array}$ \\
\hline $\mathrm{H} 24$ & + & 2.167 & 2.385 & 49.206 & 2.033 & 31.102 & $\begin{array}{l}\mathrm{O}=\mathrm{C}(\mathrm{O}[\mathrm{C} @ @](\mathrm{C})([\mathrm{H}]) \mathrm{CCC}(\mathrm{C})=\mathrm{O}) \mathrm{N}[\mathrm{C} @ @]([\mathrm{H}])(\mathrm{C} / \mathrm{C} 1=\mathrm{C} / \mathrm{C}=\mathrm{CC}=\mathrm{C} 1 \\
)[\mathrm{C} @](\mathrm{O})([\mathrm{H}]) \mathrm{CN}(\mathrm{CCCCC})[\mathrm{S}](=\mathrm{O})(=\mathrm{O}) \mathrm{C} 2=\mathrm{CC}=\mathrm{C}(\mathrm{C}=\mathrm{C} 2) \mathrm{OC}\end{array}$ \\
\hline $\mathrm{H} 25$ & + & 2.529 & 2.422 & 49.957 & 2.443 & 37.381 & $\begin{array}{l}\mathrm{O}=\mathrm{C}(\mathrm{OCCNC}(=\mathrm{O}) \mathrm{OC}(\mathrm{C})(\mathrm{C}) \mathrm{C}) \mathrm{N}[\mathrm{C} @ @]([\mathrm{H}])(\mathrm{C} / \mathrm{C} 1=\mathrm{C} / \mathrm{C}=\mathrm{CC}=\mathrm{C} 1)[\mathrm{C} \\
@](\mathrm{O})([\mathrm{H}]) \mathrm{CN}(\mathrm{CCCCC})[\mathrm{S}](=\mathrm{O})(=\mathrm{O}) \mathrm{C} 2=\mathrm{CC}=\mathrm{C}(\mathrm{C}=\mathrm{C} 2) \mathrm{OC}\end{array}$ \\
\hline $\mathrm{H} 27$ & + & 3.400 & 3.530 & 72.783 & 3.602 & 55.164 & $\begin{array}{l}\mathrm{O}=\mathrm{C}(\mathrm{OCCN} 1 \mathrm{CCNC} 1=\mathrm{O}) \mathrm{N}[\mathrm{C} @ @]([\mathrm{H}])(\mathrm{C} / \mathrm{C} 2=\mathrm{C} / \mathrm{C}=\mathrm{CC}=\mathrm{C} 2)[\mathrm{C} @](\mathrm{O}) \\
([\mathrm{H}]) \mathrm{CN}(\mathrm{CCCCC})[\mathrm{S}](=\mathrm{O})(=\mathrm{O}) \mathrm{C} 3=\mathrm{CC}=\mathrm{C}(\mathrm{C}=\mathrm{C} 3) \mathrm{OC}\end{array}$ \\
\hline $\mathrm{H} 28$ & + & 5.097 & 4.829 & 99.540 & 4.841 & 74.165 & $\begin{array}{l}\mathrm{O}=\mathrm{C}(\mathrm{OCCN} 1 \mathrm{CCOC} 1=\mathrm{O}) \mathrm{N}[\mathrm{C} @ @]([\mathrm{H}])(\mathrm{C} / \mathrm{C} 2=\mathrm{C} / \mathrm{C}=\mathrm{CC}=\mathrm{C} 2)[\mathrm{C} @](\mathrm{O}) \\
([\mathrm{H}]) \mathrm{CN}(\mathrm{CCCCC})[\mathrm{S}](=\mathrm{O})(=\mathrm{O}) \mathrm{C} 3=\mathrm{CC}=\mathrm{C}(\mathrm{C}=\mathrm{C} 3) \mathrm{OC}\end{array}$ \\
\hline $\mathrm{H} 30$ & + & 3.205 & 4.038 & 83.252 & 4.040 & 61.880 & $\begin{array}{l}\mathrm{O}=\mathrm{C}(\mathrm{OC}[\mathrm{C} @] 1([\mathrm{H}]) \mathrm{NC}(=\mathrm{O}) \mathrm{CC} 1) \mathrm{N}[\mathrm{C} @ @]([\mathrm{H}])(\mathrm{C} / \mathrm{C} 2=\mathrm{C} / \mathrm{C}=\mathrm{CC}=\mathrm{C} 2) \\
{[\mathrm{C} @](\mathrm{O})([\mathrm{H}]) \mathrm{CN}(\mathrm{CCCCC})[\mathrm{S}](=\mathrm{O})(=\mathrm{O}) \mathrm{C} 3=\mathrm{CC}=\mathrm{C}(\mathrm{C}=\mathrm{C} 3) \mathrm{OC}}\end{array}$ \\
\hline H32 & + & 1.706 & 1.746 & 36.036 & 1.842 & 28.174 & $\begin{array}{l}\mathrm{O}=\mathrm{C}(\mathrm{OCCNC}(=\mathrm{O}) \mathrm{C}([\mathrm{F}])([\mathrm{F}])[\mathrm{F}]) \mathrm{N}[\mathrm{C} @ @]([\mathrm{H}])(\mathrm{C} / \mathrm{C} 1=\mathrm{C} / \mathrm{C}=\mathrm{CC}=\mathrm{C} 1)[ \\
\mathrm{C} @](\mathrm{O})([\mathrm{H}]) \mathrm{CN}(\mathrm{CCCCC})[\mathrm{S}](=\mathrm{O})(=\mathrm{O}) \mathrm{C} 3=\mathrm{CC}=\mathrm{C} 2 \mathrm{~N}=\mathrm{C}[\mathrm{S}] \mathrm{C} 2=\mathrm{C} 3\end{array}$ \\
\hline
\end{tabular}




\begin{tabular}{|c|c|c|c|c|c|c|c|}
\hline H33 & + & 6.000 & 4.927 & 101.572 & 4.987 & 76.395 & $\begin{array}{l}\mathrm{O}=\mathrm{C}(\mathrm{OCCNC}(=\mathrm{O}) \mathrm{C}([\mathrm{Cl}])([\mathrm{Cl}])[\mathrm{H}]) \mathrm{N}[\mathrm{C} @ @]([\mathrm{H}])(\mathrm{C} / \mathrm{C} 1=\mathrm{C} / \mathrm{C}=\mathrm{CC}=\mathrm{C} 1 \\
)[\mathrm{C} @](\mathrm{O})([\mathrm{H}]) \mathrm{CN}(\mathrm{CCCCC})[\mathrm{S}](=\mathrm{O})(=\mathrm{O}) \mathrm{C} 3=\mathrm{CC}=\mathrm{C} 2 \mathrm{~N}=\mathrm{C}[\mathrm{S}] \mathrm{C} 2=\mathrm{C} 3\end{array}$ \\
\hline H35 & + & 2.889 & 2.823 & 58.229 & 2.868 & 43.906 & $\begin{array}{l}\mathrm{O}=\mathrm{C}(\mathrm{OCCNC}(=\mathrm{O}) \mathrm{OC}(\mathrm{C})(\mathrm{C}) \mathrm{C}) \mathrm{N}[\mathrm{C} @ @]([\mathrm{H}])(\mathrm{C} / \mathrm{C} 1=\mathrm{C} / \mathrm{C}=\mathrm{CC}=\mathrm{C} 1)[\mathrm{C} \\
@](\mathrm{O})([\mathrm{H}]) \mathrm{CN}(\mathrm{CCCCC})[\mathrm{S}](=\mathrm{O})(=\mathrm{O}) \mathrm{C} 3=\mathrm{CC}=\mathrm{C} 2 \mathrm{~N}=\mathrm{C}[\mathrm{S}] \mathrm{C} 2=\mathrm{C} 3\end{array}$ \\
\hline H38 & + & 4.770 & 4.901 & 101.035 & 4.925 & 75.439 & $\begin{array}{l}\mathrm{O}=\mathrm{C}(\mathrm{OCCN} 1 \mathrm{CCOC} 1=\mathrm{O}) \mathrm{N}[\mathrm{C} @ @]([\mathrm{H}])(\mathrm{C} / \mathrm{C} 2=\mathrm{C} / \mathrm{C}=\mathrm{CC}=\mathrm{C} 2)[\mathrm{C} @](\mathrm{O}) \\
([\mathrm{H}]) \mathrm{CN}(\mathrm{CCCCC})[\mathrm{S}](=\mathrm{O})(=\mathrm{O}) \mathrm{C} 4=\mathrm{CC}=\mathrm{C} 3 \mathrm{~N}=\mathrm{C}[\mathrm{S}] \mathrm{C} 3=\mathrm{C} 4\end{array}$ \\
\hline $\mathrm{H} 41$ & + & 4.000 & 3.856 & 79.502 & 4.018 & 61.540 & $\begin{array}{l}\mathrm{O}=\mathrm{C} 1 \mathrm{O}[\mathrm{C} @ @]([\mathrm{H}])(\mathrm{CN} 1 \mathrm{C}=2 \mathrm{C}=\mathrm{CC}=\mathrm{CC}=2) \mathrm{C}(=\mathrm{O}) \mathrm{N}[\mathrm{C} @ @]([\mathrm{H}])(\mathrm{C} / \\
\mathrm{C} 3=\mathrm{C} / \mathrm{C}=\mathrm{CC}=\mathrm{C} 3)[\mathrm{C} @ @](\mathrm{O})([\mathrm{H}]) \mathrm{CN}(\mathrm{CC}(\mathrm{C})(\mathrm{C})[\mathrm{H}])[\mathrm{S}](=\mathrm{O})(=\mathrm{O}) \mathrm{C} 4=\mathrm{C} \\
\mathrm{C}=\mathrm{C}(\mathrm{C}=\mathrm{C} 4) \mathrm{OC}\end{array}$ \\
\hline $\mathrm{H} 42$ & + & 4.081 & 4.049 & 83.474 & 4.085 & 62.567 & $\begin{array}{l}\mathrm{O}=\mathrm{C} 1 \mathrm{O}[\mathrm{C} @ @]([\mathrm{H}])(\mathrm{CN} 1 \mathrm{C}=2 \mathrm{C}=\mathrm{C} / \mathrm{C}=\mathrm{C}(/[\mathrm{F}]) \mathrm{C}=2) \mathrm{C}(=\mathrm{O}) \mathrm{N}[\mathrm{C} @ @]([ \\
\mathrm{H}])(\mathrm{C} / \mathrm{C} 3=\mathrm{C} / \mathrm{C}=\mathrm{CC}=\mathrm{C} 3)[\mathrm{C} @ @](\mathrm{O})([\mathrm{H}]) \mathrm{CN}(\mathrm{CC}(\mathrm{C})(\mathrm{C})[\mathrm{H}])[\mathrm{S}](=\mathrm{O})(=\mathrm{O} \\
) \mathrm{C} 4=\mathrm{CC}=\mathrm{C}(\mathrm{C}=\mathrm{C} 4) \mathrm{OC}\end{array}$ \\
\hline H45 & + & 6.097 & 4.704 & 96.967 & 4.815 & 73.763 & $\begin{array}{l}\mathrm{O}=\mathrm{C} 1 \mathrm{O}[\mathrm{C} @ @]([\mathrm{H}])(\mathrm{CN} 1 \mathrm{C}=2 \mathrm{C}=\mathrm{CC}=\mathrm{C}(\mathrm{C}=2) \mathrm{C}(\mathrm{C})=\mathrm{O}) \mathrm{C}(=\mathrm{O}) \mathrm{N}[\mathrm{C} @] @ \\
([\mathrm{H}])(\mathrm{C} / \mathrm{C} 3=\mathrm{C} / \mathrm{C}=\mathrm{CC}=\mathrm{C} 3)[\mathrm{C} @ @](\mathrm{O})([\mathrm{H}]) \mathrm{CN}(\mathrm{CC}(\mathrm{C})(\mathrm{C})[\mathrm{H}])[\mathrm{S}](=\mathrm{O})(= \\
\mathrm{O}) \mathrm{C} 4=\mathrm{CC}=\mathrm{C}(\mathrm{C}=\mathrm{C} 4) \mathrm{OC}\end{array}$ \\
\hline H46 & + & 5.398 & 4.133 & 85.206 & 4.065 & 62.264 & $\begin{array}{l}\mathrm{O}=\mathrm{C} 1 \mathrm{O}[\mathrm{C} @ @]([\mathrm{H}])(\mathrm{CN} 1 \mathrm{C}=2 \mathrm{C}=\mathrm{CC}(=\mathrm{CC}=2) \mathrm{C}(\mathrm{C})=\mathrm{O}) \mathrm{C}(=\mathrm{O}) \mathrm{N}[\mathrm{C} @{ } @] \\
([\mathrm{H}])(\mathrm{C} / \mathrm{C} 3=\mathrm{C} / \mathrm{C}=\mathrm{CC}=\mathrm{C} 3)[\mathrm{C} @ @](\mathrm{O})([\mathrm{H}]) \mathrm{CN}(\mathrm{CC}(\mathrm{C})(\mathrm{C})[\mathrm{H}])[\mathrm{S}](=\mathrm{O})(= \\
\mathrm{O}) \mathrm{C} 4=\mathrm{CC}=\mathrm{C}(\mathrm{C}=\mathrm{C} 4) \mathrm{OC}\end{array}$ \\
\hline H48 & + & 3.276 & 3.492 & 71.998 & 3.597 & 55.087 & $\begin{array}{l}\mathrm{O}=\mathrm{C} 1 \mathrm{O}[\mathrm{C} @ @]([\mathrm{H}])(\mathrm{CN} 1 \mathrm{C}=2 \mathrm{C}=\mathrm{CC}=\mathrm{CC}=2) \mathrm{C}(=\mathrm{O}) \mathrm{N}[\mathrm{C} @ @]([\mathrm{H}])(\mathrm{C} / \\
\mathrm{C} 3=\mathrm{C} / \mathrm{C}=\mathrm{CC}=\mathrm{C} 3)[\mathrm{C} @ @](\mathrm{O})([\mathrm{H}]) \mathrm{CN}(\mathrm{CC}(\mathrm{C})(\mathrm{C})[\mathrm{H}])[\mathrm{S}](=\mathrm{O})(=\mathrm{O}) \mathrm{C} 4=\mathrm{C} \\
/ \mathrm{C}=\mathrm{C}(/ \mathrm{N}) \mathrm{C}=\mathrm{C} 4\end{array}$ \\
\hline H51 & + & 4.377 & 3.515 & 72.488 & 3.336 & 51.071 & $\begin{array}{l}\mathrm{O}=\mathrm{C} 1 \mathrm{O}[\mathrm{C} @ @]([\mathrm{H}])(\mathrm{CN} 1 \mathrm{C}=2 \mathrm{C}=\mathrm{CC}=\mathrm{C}(\mathrm{C}=2) \mathrm{C}([\mathrm{F}])([\mathrm{F}])[\mathrm{F}]) \mathrm{C}(=\mathrm{O}) \mathrm{N}[\mathrm{C} \\
@ @]([\mathrm{H}])(\mathrm{C} / \mathrm{C} 3=\mathrm{C} / \mathrm{C}=\mathrm{CC}=\mathrm{C} 3)[\mathrm{C} @ @](\mathrm{O})([\mathrm{H}]) \mathrm{CN}(\mathrm{CC}(\mathrm{C})(\mathrm{C})[\mathrm{H}])[\mathrm{S}]( \\
=0)(=\mathrm{O}) \mathrm{C} 4=\mathrm{C} / \mathrm{C}=\mathrm{C}(/ \mathrm{N}) \mathrm{C}=\mathrm{C} 4\end{array}$ \\
\hline H52 & + & 3.495 & 4.339 & 89.463 & 4.395 & 67.311 & $\begin{array}{l}\mathrm{O}=\mathrm{C} 1 \mathrm{O}[\mathrm{C} @ @]([\mathrm{H}])(\mathrm{CN} 1 \mathrm{C}=2 \mathrm{C}=\mathrm{CC}=\mathrm{C}(\mathrm{C}=2) \mathrm{C}(=\mathrm{O}) \mathrm{C}) \mathrm{C}(=\mathrm{O}) \mathrm{N}[\mathrm{C} @] \\
([\mathrm{H}])(\mathrm{C} / \mathrm{C} 3=\mathrm{C} / \mathrm{C}=\mathrm{CC}=\mathrm{C} 3)[\mathrm{C} @ @](\mathrm{O})([\mathrm{H}]) \mathrm{CN}(\mathrm{CC}(\mathrm{C})(\mathrm{C})[\mathrm{H}])[\mathrm{S}](=\mathrm{O})(= \\
\mathrm{O}) \mathrm{C} 4=\mathrm{C} / \mathrm{C}=\mathrm{C}(/ \mathrm{N}) \mathrm{C}=\mathrm{C} 4\end{array}$ \\
\hline H53 & + & 3.735 & 3.769 & 77.701 & 3.645 & 55.812 & $\begin{array}{l}\mathrm{O}=\mathrm{C} 1 \mathrm{O}[\mathrm{C} @ @]([\mathrm{H}])(\mathrm{CN} 1 \mathrm{C}=2 \mathrm{C}=\mathrm{CC}(=\mathrm{CC}=2) \mathrm{C}(=\mathrm{O}) \mathrm{C}) \mathrm{C}(=\mathrm{O}) \mathrm{N}[\mathrm{C} @ \mathrm{C} @] \\
([\mathrm{H}])(\mathrm{C} / \mathrm{C} 3=\mathrm{C} / \mathrm{C}=\mathrm{CC}=\mathrm{C} 3)[\mathrm{C} @ @](\mathrm{O})([\mathrm{H}]) \mathrm{CN}(\mathrm{CC}(\mathrm{C})(\mathrm{C})[\mathrm{H}])[\mathrm{S}](=\mathrm{O})(= \\
\mathrm{O}) \mathrm{C} 4=\mathrm{C} / \mathrm{C}=\mathrm{C}(/ \mathrm{N}) \mathrm{C}=\mathrm{C} 4\end{array}$ \\
\hline H14 & + & 3.971 & 3.952 & 81.485 & 3.922 & 60.071 & $\begin{array}{l}\mathrm{O}=\mathrm{C} 1 \mathrm{O}[\mathrm{C} @ @]([\mathrm{H}])(\mathrm{CN} 1 \mathrm{C}=2 \mathrm{C}=\mathrm{C} / \mathrm{C}=\mathrm{C}(/[\mathrm{F}]) \mathrm{C}=2) \mathrm{C}(=\mathrm{O}) \mathrm{N}[\mathrm{C} @ @]([ \\
\mathrm{H}])(\mathrm{C} / \mathrm{C} 3=\mathrm{C} / \mathrm{C}=\mathrm{CC}=\mathrm{C} 3)[\mathrm{C} @ @](\mathrm{O})([\mathrm{H}]) \mathrm{CN}(\mathrm{CC}(\mathrm{C})(\mathrm{C})[\mathrm{H}])[\mathrm{S}](=\mathrm{O})(=\mathrm{O} \\
) \mathrm{C} 5=\mathrm{CC}=\mathrm{C} 4 \mathrm{OCOC} 4=\mathrm{C} 5\end{array}$ \\
\hline H57 & + & 5.222 & 4.607 & 94.978 & 4.653 & 71.267 & $\begin{array}{l}\mathrm{O}=\mathrm{C} 1 \mathrm{O}[\mathrm{C} @ @]([\mathrm{H}])(\mathrm{CN} 1 \mathrm{C}=2 \mathrm{C}=\mathrm{CC}=\mathrm{C}(\mathrm{C}=2) \mathrm{C}(=\mathrm{O}) \mathrm{C}) \mathrm{C}(=\mathrm{O}) \mathrm{N}[\mathrm{C} @] @ \\
([\mathrm{H}])(\mathrm{C} / \mathrm{C} 3=\mathrm{C} / \mathrm{C}=\mathrm{CC}=\mathrm{C} 3)[\mathrm{C} @ @](\mathrm{O})([\mathrm{H}]) \mathrm{CN}(\mathrm{CC}(\mathrm{C})(\mathrm{C})[\mathrm{H}])[\mathrm{S}](=\mathrm{O})(= \\
\mathrm{O}) \mathrm{C} 5=\mathrm{CC}=\mathrm{C} 4 \mathrm{OCOC} 4=\mathrm{C} 5\end{array}$ \\
\hline H60 & + & 3.465 & 3.962 & 81.693 & 3.953 & 60.547 & $\begin{array}{l}\mathrm{O}=\mathrm{C} 1 \mathrm{O}[\mathrm{C} @ @]([\mathrm{H}])(\mathrm{CN} 1 \mathrm{C}=2 \mathrm{C}=\mathrm{CC}([\mathrm{F}])=\mathrm{C}([\mathrm{F}]) \mathrm{C}=2) \mathrm{C}(=\mathrm{O}) \mathrm{N}[\mathrm{C} @ @] \\
([\mathrm{H}])(\mathrm{C} / \mathrm{C} 3=\mathrm{C} / \mathrm{C}=\mathrm{CC}=\mathrm{C} 3)[\mathrm{C} @ @](\mathrm{O})([\mathrm{H}]) \mathrm{CN}(\mathrm{CC}(\mathrm{C})(\mathrm{C})[\mathrm{H}])[\mathrm{S}](=\mathrm{O})(= \\
\mathrm{O}) \mathrm{C} 4=\mathrm{C} / \mathrm{C}=\mathrm{C}(/ \mathrm{OC}) \mathrm{C}(\mathrm{C})=\mathrm{C} 4\end{array}$ \\
\hline H66 & + & 2.420 & 2.508 & 51.746 & 2.567 & 39.277 & $\begin{array}{l}\mathrm{O}=\mathrm{C} 1 \mathrm{O}[\mathrm{C} @ @]([\mathrm{H}])(\mathrm{CN} 1 \mathrm{C}=2 \mathrm{C}=\mathrm{CC}=\mathrm{CC}=2) \mathrm{C}(=\mathrm{O}) \mathrm{N}[\mathrm{C} @ @]([\mathrm{H}])(\mathrm{C} / \\
\mathrm{C} 3=\mathrm{C} / \mathrm{C}=\mathrm{CC}=\mathrm{C} 3)[\mathrm{C} @ @](\mathrm{O})([\mathrm{H}]) \mathrm{CN}(\mathrm{CC}(\mathrm{C})(\mathrm{C})[\mathrm{H}])[\mathrm{S}](=\mathrm{O})(=\mathrm{O}) \mathrm{C} 4=\mathrm{C} \\
\mathrm{C}=\mathrm{C}(\mathrm{C}=\mathrm{C} 4) \mathrm{OC}([\mathrm{F}])([\mathrm{F}])[\mathrm{F}]\end{array}$ \\
\hline H67 & + & 3.076 & 2.785 & 57.449 & 2.614 & 40.002 & $\begin{array}{l}\mathrm{O}=\mathrm{C} 1 \mathrm{O}[\mathrm{C} @ @]([\mathrm{H}])(\mathrm{CN} 1 \mathrm{C}=2 \mathrm{C}=\mathrm{CC}(=\mathrm{CC}=2) \mathrm{C}(=\mathrm{O}) \mathrm{C}) \mathrm{C}(=\mathrm{O}) \mathrm{N}[\mathrm{C} @ @] \\
([\mathrm{H}])(\mathrm{C} / \mathrm{C} 3=\mathrm{C} / \mathrm{C}=\mathrm{CC}=\mathrm{C} 3)[\mathrm{C} @ @](\mathrm{O})([\mathrm{H}]) \mathrm{CN}(\mathrm{CC}(\mathrm{C})(\mathrm{C})[\mathrm{H}])[\mathrm{S}](=\mathrm{O})(= \\
\mathrm{O}) \mathrm{C} 4=\mathrm{CC}=\mathrm{C}(\mathrm{C}=\mathrm{C} 4) \mathrm{OC}([\mathrm{F}])([\mathrm{F}])[\mathrm{F}]\end{array}$ \\
\hline H68 & + & 3.590 & 3.407 & 70.253 & 3.433 & 52.567 & $\begin{array}{l}\mathrm{O}=\mathrm{C} 1 \mathrm{O}[\mathrm{C} @ @]([\mathrm{H}])(\mathrm{CN} 1 \mathrm{C}=2 \mathrm{C}=\mathrm{C} / \mathrm{C}=\mathrm{C} / /[\mathrm{F}]) \mathrm{C}=2) \mathrm{C}(=\mathrm{O}) \mathrm{N}[\mathrm{C} @(\mathrm{C})([ \\
\mathrm{H}])(\mathrm{C} / \mathrm{C} 3=\mathrm{C} / \mathrm{C}=\mathrm{CC}=\mathrm{C} 3)[\mathrm{C} @ @](\mathrm{O})([\mathrm{H}]) \mathrm{CN}(\mathrm{C}[\mathrm{C} @ @] 4([\mathrm{H}]) \mathrm{CC})[\mathrm{S}](\end{array}$ \\
\hline
\end{tabular}




\begin{tabular}{|c|c|c|c|c|c|c|c|}
\hline & & & & & & & $=0)(=0) C 5=C C=C(C=C 5) O C$ \\
\hline H69 & + & 3.237 & 3.432 & 70.764 & 3.431 & 52.529 & $\begin{array}{l}\mathrm{O}=\mathrm{C} 1 \mathrm{O}[\mathrm{C} @ @]([\mathrm{H}])(\mathrm{CN} 1 \mathrm{C}=2 \mathrm{C}=\mathrm{CC}([\mathrm{F}])=\mathrm{C}([\mathrm{F}]) \mathrm{C}=2) \mathrm{C}(=\mathrm{O}) \mathrm{N}[\mathrm{C} @ @] \\
([\mathrm{H}])(\mathrm{C} / \mathrm{C} 3=\mathrm{C} / \mathrm{C}=\mathrm{CC}=\mathrm{C} 3)[\mathrm{C} @ @](\mathrm{O})([\mathrm{H}]) \mathrm{CN}(\mathrm{C}[\mathrm{C} @ @] 4([\mathrm{H}]) \mathrm{CC} 4)[\mathrm{S} \\
](=O)(=O) \mathrm{C} 5=\mathrm{CC}=\mathrm{C}(\mathrm{C}=\mathrm{C} 5) \mathrm{OC}\end{array}$ \\
\hline $\mathrm{H} 71$ & + & 0.622 & 0.654 & 13.536 & 0.714 & 10.862 & $\begin{array}{l}\mathrm{O}=\mathrm{C} 1 \mathrm{O}[\mathrm{C} @ @]([\mathrm{H}])(\mathrm{CN} 1 \mathrm{C}=2 \mathrm{C}=\mathrm{CC}=\mathrm{CC}=2) \mathrm{C}(=\mathrm{O}) \mathrm{N}[\mathrm{C} @ @]([\mathrm{H}])(\mathrm{C} / \\
\mathrm{C} 3=\mathrm{C} / \mathrm{C}=\mathrm{CC}=\mathrm{C} 3)[\mathrm{C} @ @](\mathrm{O})([\mathrm{H}]) \mathrm{CN}(\mathrm{C} / \mathrm{C} 4=\mathrm{C} / \mathrm{C}=\mathrm{C}[\mathrm{S}] 4)[\mathrm{S}](=\mathrm{O})(=\mathrm{O}) \\
\mathrm{C} 5=\mathrm{CC}=\mathrm{CC}(=\mathrm{C} 5) \mathrm{OC}\end{array}$ \\
\hline $\mathrm{H} 76$ & + & 0.775 & 0.857 & 17.725 & 0.892 & 13.592 & $\begin{array}{l}\mathrm{O}=\mathrm{C} 1 \mathrm{O}[\mathrm{C} @ @]([\mathrm{H}])(\mathrm{CN} 1 \mathrm{C}=2 \mathrm{C}=\mathrm{CC}(=\mathrm{CC}=2) \mathrm{C}(=\mathrm{O}) \mathrm{C}) \mathrm{C}(=\mathrm{O}) \mathrm{N}[\mathrm{C} @ @] \\
([\mathrm{H}])(\mathrm{C} / \mathrm{C} 3=\mathrm{C} / \mathrm{C}=\mathrm{CC}=\mathrm{C} 3)[\mathrm{C} @ @](\mathrm{O})([\mathrm{H}]) \mathrm{CN}(\mathrm{C} / \mathrm{C} 4=\mathrm{C} / \mathrm{C}=\mathrm{C}[\mathrm{S}] 4)[\mathrm{S}]( \\
=\mathrm{O})(=\mathrm{O}) \mathrm{C} 5=\mathrm{CC}([\mathrm{F}])=\mathrm{C}([\mathrm{F}]) \mathrm{C}=\mathrm{C} 5[\mathrm{~F}]\end{array}$ \\
\hline $\mathrm{H} 77$ & + & 1.377 & 1.065 & 22.011 & 1.133 & 17.296 & $\begin{array}{l}\mathrm{O}=\mathrm{C} 1 \mathrm{O}[\mathrm{C} @ @]([\mathrm{H}])(\mathrm{CN} 1 \mathrm{C}=2 \mathrm{C}=\mathrm{CC}=\mathrm{CC}=2) \mathrm{C}(=\mathrm{O}) \mathrm{N}[\mathrm{C} @ @]([\mathrm{H}])(\mathrm{C} / \\
\mathrm{C} 3=\mathrm{C} / \mathrm{C}=\mathrm{CC}=\mathrm{C} 3)[\mathrm{C} @ @](\mathrm{O})([\mathrm{H}]) \mathrm{CN}(\mathrm{C}[\mathrm{C} @ @] 4([\mathrm{H}]) \mathrm{CCCO} 4)[\mathrm{S}](=\mathrm{O} \\
)(=\mathrm{O}) \mathrm{C} 5=\mathrm{CC}=\mathrm{CC}(=\mathrm{C} 5) \mathrm{OC}\end{array}$ \\
\hline $\mathrm{H} 78$ & + & 0.824 & 1.258 & 25.982 & 1.200 & 18.323 & $\begin{array}{l}\mathrm{O}=\mathrm{C} 1 \mathrm{O}[\mathrm{C} @ @]([\mathrm{H}])(\mathrm{CN} 1 \mathrm{C}=2 \mathrm{C}=\mathrm{C} / \mathrm{C}=\mathrm{C}(/[\mathrm{F}]) \mathrm{C}=2) \mathrm{C}(=\mathrm{O}) \mathrm{N}[\mathrm{C} @ @]([ \\
\mathrm{H}])(\mathrm{C} / \mathrm{C} 3=\mathrm{C} / \mathrm{C}=\mathrm{CC}=\mathrm{C} 3)[\mathrm{C} @ @](\mathrm{O})([\mathrm{H}]) \mathrm{CN}(\mathrm{C}[\mathrm{C} @ @] 4([\mathrm{H}]) \mathrm{CCCO})[ \\
\mathrm{S}](=\mathrm{O})(=\mathrm{O}) \mathrm{C} 5=\mathrm{CC}=\mathrm{CC}(=\mathrm{C} 5) \mathrm{OC}\end{array}$ \\
\hline $\mathrm{H} 79$ & + & 3.686 & 3.759 & 77.513 & 3.855 & 59.044 & $\begin{array}{l}\mathrm{O}=\mathrm{C} 1 \mathrm{O}[\mathrm{C} @ @]([\mathrm{H}])(\mathrm{CN} 1 \mathrm{C}=2 \mathrm{C}=\mathrm{CC}=\mathrm{CC}=2) \mathrm{C}(=\mathrm{O}) \mathrm{N}[\mathrm{C} @ @]([\mathrm{H}])(\mathrm{C} / \\
\mathrm{C} 3=\mathrm{C} / \mathrm{C}=\mathrm{CC}=\mathrm{C} 3)[\mathrm{C} @ @](\mathrm{O})([\mathrm{H}]) \mathrm{CN}(\mathrm{CC}(\mathrm{C})(\mathrm{C})[\mathrm{H}])[\mathrm{S}](=\mathrm{O})(=\mathrm{O}) \mathrm{C} 5=\mathrm{C} \\
\mathrm{C}=\mathrm{C} 4 \mathrm{OCOC} 4=\mathrm{C} 5\end{array}$ \\
\hline $\mathrm{H} 80$ & + & 4.481 & 4.305 & 88.744 & 4.427 & 67.806 & $\begin{array}{l}\mathrm{O}=\mathrm{C} 1 \mathrm{O}[\mathrm{C} @ @]([\mathrm{H}])(\mathrm{CN} 1 \mathrm{C}=2 \mathrm{C}=\mathrm{CC}=\mathrm{CC}=2) \mathrm{C}(=\mathrm{O}) \mathrm{N}[\mathrm{C} @ @]([\mathrm{H}])(\mathrm{C} / \\
\mathrm{C} 3=\mathrm{C} / \mathrm{C}=\mathrm{CC}=\mathrm{C} 3)[\mathrm{C} @ @](\mathrm{O})([\mathrm{H}]) \mathrm{CN}(\mathrm{CC}(\mathrm{C})(\mathrm{C})[\mathrm{H}])[\mathrm{S}](=\mathrm{O})(=\mathrm{O}) \mathrm{C} 5=\mathrm{C} \\
\mathrm{C}=\mathrm{C} 4 \mathrm{~N}=\mathrm{C}[\mathrm{S}] \mathrm{C} 4=\mathrm{C} 5\end{array}$ \\
\hline H81 & + & 3.599 & 3.358 & 69.241 & 3.497 & 53.548 & $\begin{array}{l}\mathrm{O}=\mathrm{C} 1 \mathrm{O}[\mathrm{C} @ @]([\mathrm{H}])(\mathrm{CN} 1 \mathrm{C}=2 \mathrm{C}=\mathrm{CC}=\mathrm{CC}=2) \mathrm{C}(=\mathrm{O}) \mathrm{N}[\mathrm{C} @ @]([\mathrm{H}])(\mathrm{C} / \\
\mathrm{C} 3=\mathrm{C} / \mathrm{C}=\mathrm{CC}=\mathrm{C} 3)[\mathrm{C} @ @](\mathrm{O})([\mathrm{H}]) \mathrm{CN}(\mathrm{CC}(\mathrm{C})(\mathrm{C})[\mathrm{H}])[\mathrm{S}](=\mathrm{O})(=\mathrm{O}) \mathrm{C} 4=\mathrm{C} \\
\mathrm{C}=\mathrm{C}(\mathrm{C}=\mathrm{C} 4) \mathrm{CO}\end{array}$ \\
\hline $\mathrm{H} 83$ & + & 2.248 & 2.278 & 47.004 & 2.306 & 35.288 & $\begin{array}{l}\mathrm{O}=\mathrm{C} 1 \mathrm{O}[\mathrm{C} @ @]([\mathrm{H}])(\mathrm{CN} 1 \mathrm{C}=2 \mathrm{C}=\mathrm{CC}=\mathrm{CC}=2 \mathrm{O}) \mathrm{C}(=\mathrm{O}) \mathrm{N}[\mathrm{C} @ @]([\mathrm{H}])(\mathrm{C} \\
/ \mathrm{C} 3=\mathrm{C} / \mathrm{C}=\mathrm{CC}=\mathrm{C} 3)[\mathrm{C} @ @](\mathrm{O})([\mathrm{H}]) \mathrm{CN}(\mathrm{CC}(\mathrm{C})(\mathrm{C})[\mathrm{H}])[\mathrm{S}](=\mathrm{O})(=\mathrm{O}) \mathrm{C} 4= \\
\mathrm{CC}=\mathrm{C}(\mathrm{C}=\mathrm{C} 4) \mathrm{OC}\end{array}$ \\
\hline H85 & + & 3.866 & 3.662 & 75.498 & 3.646 & 55.832 & $\begin{array}{l}\mathrm{O}=\mathrm{C} 1 \mathrm{O}[\mathrm{C} @ @]([\mathrm{H}])(\mathrm{CN} 1 \mathrm{C}=2 \mathrm{C}=\mathrm{CC}=\mathrm{CC}=2 \mathrm{C}([\mathrm{F}])([\mathrm{F}])[\mathrm{F}]) \mathrm{C}(=\mathrm{O}) \mathrm{N}[\mathrm{C} \\
@ @]([\mathrm{H}])(\mathrm{C} / \mathrm{C} 3=\mathrm{C} / \mathrm{C}=\mathrm{CC}=\mathrm{C} 3)[\mathrm{C} @ @](\mathrm{O})([\mathrm{H}]) \mathrm{CN}(\mathrm{CC}(\mathrm{C})(\mathrm{C})[\mathrm{H}])[\mathrm{S}]( \\
=\mathrm{O})(=\mathrm{O}) \mathrm{C} 4=\mathrm{C} / \mathrm{C}=\mathrm{C}(/ \mathrm{N}) \mathrm{C}=\mathrm{C} 4\end{array}$ \\
\hline H86 & + & 4.066 & 4.026 & 83.003 & 4.067 & 62.284 & $\begin{array}{l}\mathrm{O}=\mathrm{C} 1 \mathrm{O}[\mathrm{C} @ @]([\mathrm{H}])(\mathrm{CN} 1 \mathrm{C}=2 \mathrm{C}=\mathrm{CC}=\mathrm{CC}=2 \mathrm{C}([\mathrm{F}])([\mathrm{F}])[\mathrm{F}]) \mathrm{C}(=\mathrm{O}) \mathrm{N}[\mathrm{C} \\
@ @]([\mathrm{H}])(\mathrm{C} / \mathrm{C} 3=\mathrm{C} / \mathrm{C}=\mathrm{CC}=\mathrm{C} 3)[\mathrm{C} @ @](\mathrm{O})([\mathrm{H}]) \mathrm{CN}(\mathrm{CC}(\mathrm{C})(\mathrm{C})[\mathrm{H}])[\mathrm{S}]( \\
=\mathrm{O})(=\mathrm{O}) \mathrm{C} 4=\mathrm{CC}=\mathrm{C}(\mathrm{C}=\mathrm{C} 4) \mathrm{OC}\end{array}$ \\
\hline H87 & + & 3.848 & 3.929 & 81.013 & 3.904 & 59.789 & $\begin{array}{l}\mathrm{O}=\mathrm{C} 1 \mathrm{O}[\mathrm{C} @ @]([\mathrm{H}])(\mathrm{CN} 1 \mathrm{C}=2 \mathrm{C}=\mathrm{CC}=\mathrm{CC}=2 \mathrm{C}([\mathrm{F}])([\mathrm{F}])[\mathrm{F}]) \mathrm{C}(=\mathrm{O}) \mathrm{N}[\mathrm{C} \\
@ @]([\mathrm{H}])(\mathrm{C} / \mathrm{C} 3=\mathrm{C} / \mathrm{C}=\mathrm{CC}=\mathrm{C} 3)[\mathrm{C} @ @](\mathrm{O})([\mathrm{H}]) \mathrm{CN}(\mathrm{CC}(\mathrm{C})(\mathrm{C})[\mathrm{H}])[\mathrm{S}]( \\
=\mathrm{O})(=\mathrm{O}) \mathrm{C} 5=\mathrm{CC}=\mathrm{C} 40 \mathrm{OCOC} 4=\mathrm{C} 5\end{array}$ \\
\hline H88 & + & 4.013 & 4.475 & 92.244 & 4.475 & 68.550 & $\begin{array}{l}\mathrm{O}=\mathrm{C} 1 \mathrm{O}[\mathrm{C} @ @]([\mathrm{H}])(\mathrm{CN} 1 \mathrm{C}=2 \mathrm{C}=\mathrm{CC}=\mathrm{CC}=2 \mathrm{C}([\mathrm{F}])([\mathrm{F}])[\mathrm{F}]) \mathrm{C}(=\mathrm{O}) \mathrm{N}[\mathrm{C} \\
@ @]([\mathrm{H}])(\mathrm{C} / \mathrm{C} 3=\mathrm{C} / \mathrm{C}=\mathrm{CC}=\mathrm{C} 3)[\mathrm{C} @ @](\mathrm{O})([\mathrm{H}]) \mathrm{CN}(\mathrm{CC}(\mathrm{C})(\mathrm{C})[\mathrm{H}])[\mathrm{S}]( \\
=\mathrm{O})(=\mathrm{O}) \mathrm{C} 5=\mathrm{CC}=\mathrm{C} 4 \mathrm{~N}=\mathrm{C}[\mathrm{S}] \mathrm{C} 4=\mathrm{C} 5\end{array}$ \\
\hline H89 & + & 3.629 & 3.528 & 72.741 & 3.546 & 54.293 & $\begin{array}{l}\mathrm{O}=\mathrm{C} 1 \mathrm{O}[\mathrm{C} @ @]([\mathrm{H}])(\mathrm{CN} 1 \mathrm{C}=2 \mathrm{C}=\mathrm{CC}=\mathrm{CC}=2 \mathrm{C}([\mathrm{F}])([\mathrm{F}])[\mathrm{F}]) \mathrm{C}(=\mathrm{O}) \mathrm{N}[\mathrm{C} \\
@ @]([\mathrm{H}])(\mathrm{C} / \mathrm{C} 3=\mathrm{C} / \mathrm{C}=\mathrm{CC}=\mathrm{C} 3)[\mathrm{C} @ @](\mathrm{O})([\mathrm{H}]) \mathrm{CN}(\mathrm{CC}(\mathrm{C})(\mathrm{C})[\mathrm{H}])[\mathrm{S}]( \\
=\mathrm{O})(=\mathrm{O}) \mathrm{C} 4=\mathrm{CC}=\mathrm{C}(\mathrm{C}=\mathrm{C} 4) \mathrm{CO}\end{array}$ \\
\hline H91 & + & 4.201 & 3.794 & 78.235 & 3.806 & 58.280 & $\begin{array}{l}\mathrm{O}=\mathrm{C} 1 \mathrm{O}[\mathrm{C} @ @]([\mathrm{H}])(\mathrm{CN} 1 \mathrm{C}=2 \mathrm{C}=\mathrm{CC}([\mathrm{F}])=\mathrm{CC}=2[\mathrm{~F}]) \mathrm{C}(=\mathrm{O}) \mathrm{N}[\mathrm{C} @ @]([ \\
\mathrm{H}])(\mathrm{C} / \mathrm{C} 3=\mathrm{C} / \mathrm{C}=\mathrm{CC}=\mathrm{C} 3)[\mathrm{C} @ @](\mathrm{O})([\mathrm{H}]) \mathrm{CN}(\mathrm{CC}(\mathrm{C})(\mathrm{C})[\mathrm{H}])[\mathrm{S}](=\mathrm{O})(=\mathrm{O} \\
) \mathrm{C} 4=\mathrm{CC}=\mathrm{C}(\mathrm{C}=\mathrm{C} 4) \mathrm{OC}\end{array}$ \\
\hline
\end{tabular}




\begin{tabular}{|c|c|c|c|c|c|c|c|}
\hline H93 & + & 3.824 & 4.243 & 87.477 & 4.214 & 64.545 & $\begin{array}{l}\mathrm{O}=\mathrm{C} 1 \mathrm{O}[\mathrm{C} @ @]([\mathrm{H}])(\mathrm{CN} 1 \mathrm{C}=2 \mathrm{C}=\mathrm{CC}([\mathrm{F}])=\mathrm{CC}=2[\mathrm{~F}]) \mathrm{C}(=\mathrm{O}) \mathrm{N}[\mathrm{C} @ @]([ \\
\mathrm{H}])(\mathrm{C} / \mathrm{C} 3=\mathrm{C} / \mathrm{C}=\mathrm{CC}=\mathrm{C} 3)[\mathrm{C} @ @](\mathrm{O})([\mathrm{H}]) \mathrm{CN}(\mathrm{CC}(\mathrm{C})(\mathrm{C})[\mathrm{H}])[\mathrm{S}](=\mathrm{O})(=\mathrm{O} \\
) \mathrm{C} 5=\mathrm{CC}=\mathrm{C} 4 \mathrm{~N}=\mathrm{C}[\mathrm{S}] \mathrm{C} 4=\mathrm{C} 5\end{array}$ \\
\hline H95 & + & 3.349 & 3.406 & 70.227 & 3.483 & 53.332 & $\begin{array}{l}\mathrm{O}=\mathrm{C} 1 \mathrm{O}[\mathrm{C} @ @]([\mathrm{H}])(\mathrm{CN} 1 \mathrm{C}=2 \mathrm{C}=\mathrm{CC}([\mathrm{F}])=\mathrm{CC}=2) \mathrm{C}(=\mathrm{O}) \mathrm{N}[\mathrm{C} @ @]([\mathrm{H}]) \\
(\mathrm{C} / \mathrm{C} 3=\mathrm{C} / \mathrm{C}=\mathrm{CC}=\mathrm{C} 3)[\mathrm{C} @ @](\mathrm{O})([\mathrm{H}]) \mathrm{CN}(\mathrm{CC}(\mathrm{C})(\mathrm{C})[\mathrm{H}])[\mathrm{S}](=\mathrm{O})(=\mathrm{O}) \mathrm{C} \\
4=\mathrm{C} / \mathrm{C}=\mathrm{C}(/ \mathrm{N}) \mathrm{C}=\mathrm{C} 4\end{array}$ \\
\hline H97 & + & 3.777 & 3.673 & 75.743 & 3.741 & 57.289 & $\begin{array}{l}\mathrm{O}=\mathrm{C} 1 \mathrm{O}[\mathrm{C} @ @]([\mathrm{H}])(\mathrm{CN} 1 \mathrm{C}=2 \mathrm{C}=\mathrm{CC}([\mathrm{F}])=\mathrm{CC}=2) \mathrm{C}(=\mathrm{O}) \mathrm{N}[\mathrm{C} @ @]([\mathrm{H}]) \\
(\mathrm{C} / \mathrm{C} 3=\mathrm{C} / \mathrm{C}=\mathrm{CC}=\mathrm{C} 3)[\mathrm{C} @ @](\mathrm{O})([\mathrm{H}]) \mathrm{CN}(\mathrm{CC}(\mathrm{C})(\mathrm{C})[\mathrm{H}])[\mathrm{S}](=\mathrm{O})(=\mathrm{O}) \mathrm{C} \\
5=\mathrm{CC}=\mathrm{C} 4 \mathrm{OCOC} 4=\mathrm{C} 5\end{array}$ \\
\hline H98 & + & 3.876 & 4.219 & 86.974 & 4.312 & 66.050 & $\begin{array}{l}\mathrm{O}=\mathrm{C} 1 \mathrm{O}[\mathrm{C} @ @]([\mathrm{H}])(\mathrm{CN} 1 \mathrm{C}=2 \mathrm{C}=\mathrm{CC}([\mathrm{F}])=\mathrm{CC}=2) \mathrm{C}(=\mathrm{O}) \mathrm{N}[\mathrm{C} @ @]([\mathrm{H}]) \\
(\mathrm{C} / \mathrm{C} 3=\mathrm{C} / \mathrm{C}=\mathrm{CC}=\mathrm{C} 3)[\mathrm{C} @ @](\mathrm{O})([\mathrm{H}]) \mathrm{CN}(\mathrm{CC}(\mathrm{C})(\mathrm{C})[\mathrm{H}])[\mathrm{S}](=\mathrm{O})(=\mathrm{O}) \mathrm{C} \\
5=\mathrm{CC}=\mathrm{C} 4 \mathrm{~N}=\mathrm{C}[\mathrm{S}] \mathrm{C} 4=\mathrm{C} 5\end{array}$ \\
\hline H100 & + & 4.137 & 4.581 & 94.448 & 4.474 & 68.530 & $\begin{array}{l}\mathrm{O}=\mathrm{C} 1 \mathrm{O}[\mathrm{C} @ @]([\mathrm{H}])(\mathrm{CN} 1 \mathrm{C}=2 \mathrm{C}=\mathrm{CC}(=\mathrm{CC}=2) \mathrm{C}(\mathrm{C})=\mathrm{O}) \mathrm{C}(=\mathrm{O}) \mathrm{N}[\mathrm{C} @] \\
([\mathrm{H}])(\mathrm{C} / \mathrm{C} 3=\mathrm{C} / \mathrm{C}=\mathrm{CC}=\mathrm{C} 3)[\mathrm{C} @ @](\mathrm{O})([\mathrm{H}]) \mathrm{CN}(\mathrm{CC}(\mathrm{C})(\mathrm{C})[\mathrm{H}])[\mathrm{S}](=\mathrm{O})(= \\
\mathrm{O}) \mathrm{C} 5=\mathrm{CC}=\mathrm{C} 4 \mathrm{~N}=\mathrm{C}[\mathrm{S}] \mathrm{C} 4=\mathrm{C} 5\end{array}$ \\
\hline H101 & + & 3.499 & 3.635 & 74.944 & 3.544 & 54.273 & $\begin{array}{l}\mathrm{O}=\mathrm{C} 1 \mathrm{O}[\mathrm{C} @ @]([\mathrm{H}])(\mathrm{CN} 1 \mathrm{C}=2 \mathrm{C}=\mathrm{CC}(=\mathrm{CC}=2) \mathrm{C}(\mathrm{C})=\mathrm{O}) \mathrm{C}(=\mathrm{O}) \mathrm{N}[\mathrm{C} @] \\
([\mathrm{H}])(\mathrm{C} / \mathrm{C} 3=\mathrm{C} / \mathrm{C}=\mathrm{CC}=\mathrm{C} 3)[\mathrm{C} @ @](\mathrm{O})([\mathrm{H}]) \mathrm{CN}(\mathrm{CC}(\mathrm{C})(\mathrm{C})[\mathrm{H}])[\mathrm{S}](=\mathrm{O})(= \\
\mathrm{O}) \mathrm{C} 4=\mathrm{CC}=\mathrm{C}(\mathrm{C}=\mathrm{C} 4) \mathrm{CO}\end{array}$ \\
\hline H102 & + & 4.097 & 4.497 & 92.716 & 4.494 & 68.833 & $\begin{array}{l}\mathrm{O}=\mathrm{C} 1 \mathrm{O}[\mathrm{C} @ @]([\mathrm{H}])(\mathrm{CN} 1 \mathrm{C}=2 \mathrm{C}=\mathrm{C} / \mathrm{C}=\mathrm{C}(/[\mathrm{F}]) \mathrm{C}=2) \mathrm{C}(=\mathrm{O}) \mathrm{N}[\mathrm{C} @ @]([ \\
\mathrm{H}])(\mathrm{C} / \mathrm{C} 3=\mathrm{C} / \mathrm{C}=\mathrm{CC}=\mathrm{C} 3)[\mathrm{C} @ @](\mathrm{O})([\mathrm{H}]) \mathrm{CN}(\mathrm{CC}(\mathrm{C})(\mathrm{C})[\mathrm{H}])[\mathrm{S}](=\mathrm{O})(=\mathrm{O} \\
) \mathrm{C} 5=\mathrm{CC}=\mathrm{C} 4 \mathrm{~N}=\mathrm{C}[\mathrm{S}] \mathrm{C} 4=\mathrm{C} 5\end{array}$ \\
\hline H104 & + & 3.635 & 4.522 & 93.226 & 4.491 & 68.795 & $\begin{array}{l}\mathrm{O}=\mathrm{C} 1 \mathrm{O}[\mathrm{C} @ @]([\mathrm{H}])(\mathrm{CN} 1 \mathrm{C}=2 \mathrm{C}=\mathrm{CC}([\mathrm{F}])=\mathrm{C}([\mathrm{F}]) \mathrm{C}=2) \mathrm{C}(=\mathrm{O}) \mathrm{N}[\mathrm{C} @ @] \\
([\mathrm{H}])(\mathrm{C} / \mathrm{C} 3=\mathrm{C} / \mathrm{C}=\mathrm{CC}=\mathrm{C} 3)[\mathrm{C} @ @](\mathrm{O})([\mathrm{H}]) \mathrm{CN}(\mathrm{CC}(\mathrm{C})(\mathrm{C})[\mathrm{H}])[\mathrm{S}](=\mathrm{O})(= \\
\mathrm{O}) \mathrm{C} 5=\mathrm{CC}=\mathrm{C} 4 \mathrm{~N}=\mathrm{C}[\mathrm{S}] \mathrm{C} 4=\mathrm{C} 5\end{array}$ \\
\hline H105 & + & 3.481 & 3.575 & 73.723 & 3.562 & 54.538 & $\begin{array}{l}\mathrm{O}=\mathrm{C} 1 \mathrm{O}[\mathrm{C} @ @]([\mathrm{H}])(\mathrm{CN} 1 \mathrm{C}=2 \mathrm{C}=\mathrm{CC}([\mathrm{F}])=\mathrm{C}([\mathrm{F}]) \mathrm{C}=2) \mathrm{C}(=\mathrm{O}) \mathrm{N}[\mathrm{C} @ @] \\
([\mathrm{H}])(\mathrm{C} / \mathrm{C} 3=\mathrm{C} / \mathrm{C}=\mathrm{CC}=\mathrm{C} 3)[\mathrm{C} @ @](\mathrm{O})([\mathrm{H}]) \mathrm{CN}(\mathrm{CC}(\mathrm{C})(\mathrm{C})[\mathrm{H}])[\mathrm{S}](=\mathrm{O})(= \\
\mathrm{O}) \mathrm{C} 4=\mathrm{CC}=\mathrm{C}(\mathrm{C}=\mathrm{C} 4) \mathrm{CO}\end{array}$ \\
\hline H106 & + & 4.796 & 4.328 & 89.235 & 4.165 & 63.789 & $\begin{array}{l}\mathrm{O}=\mathrm{C} 1 \mathrm{O}[\mathrm{C} @ @]([\mathrm{H}])(\mathrm{CN} 1 \mathrm{C}=2 \mathrm{C}=\mathrm{CC}=\mathrm{C}(\mathrm{C}=2) \mathrm{C}([\mathrm{F}])([\mathrm{F}])[\mathrm{F}]) \mathrm{C}(=\mathrm{O}) \mathrm{N}[\mathrm{C} \\
@ @]([\mathrm{H}])(\mathrm{C} / \mathrm{C} 3=\mathrm{C} / \mathrm{C}=\mathrm{CC}=\mathrm{C} 3)[\mathrm{C} @ @](\mathrm{O})([\mathrm{H}]) \mathrm{CN}(\mathrm{CC}(\mathrm{C})(\mathrm{C})[\mathrm{H}])[\mathrm{S}]( \\
=0)(=\mathrm{O}) \mathrm{C} 5=\mathrm{CC}=\mathrm{C} 4 \mathrm{~N}=\mathrm{C}[\mathrm{S}] \mathrm{C} 4=\mathrm{C} 5\end{array}$ \\
\hline H108 & + & 3.648 & 3.418 & 70.485 & 3.498 & 53.567 & $\begin{array}{l}\mathrm{O}=\mathrm{C} 1 \mathrm{O}[\mathrm{C} @ @]([\mathrm{H}])(\mathrm{CN} 1 \mathrm{C}=2 \mathrm{C}=\mathrm{CC}=\mathrm{C}(\mathrm{C}=2) \mathrm{OC}([\mathrm{F}])([\mathrm{F}])[\mathrm{F}]) \mathrm{C}(=\mathrm{O}) \mathrm{N} \\
{[\mathrm{C} @ @]([\mathrm{H}])(\mathrm{C} / \mathrm{C} 3=\mathrm{C} / \mathrm{C}=\mathrm{CC}=\mathrm{C} 3)[\mathrm{C} @ @](\mathrm{O})([\mathrm{H}]) \mathrm{CN}(\mathrm{CC}(\mathrm{C})(\mathrm{C})[\mathrm{H}])[\mathrm{S}} \\
](=\mathrm{O})(=\mathrm{O}) \mathrm{C} 4=\mathrm{C} / \mathrm{C}=\mathrm{C}(/ \mathrm{N}) \mathrm{C}=\mathrm{C} 4\end{array}$ \\
\hline H109 & + & 3.886 & 3.783 & 77.989 & 3.919 & 60.019 & $\begin{array}{l}\mathrm{O}=\mathrm{C} 1 \mathrm{O}[\mathrm{C} @ @]([\mathrm{H}])(\mathrm{CN} 1 \mathrm{C}=2 \mathrm{C}=\mathrm{CC}=\mathrm{C}(\mathrm{C}=2) \mathrm{OC}([\mathrm{F}])([\mathrm{F}])[\mathrm{F}]) \mathrm{C}(=\mathrm{O}) \mathrm{N} \\
{[\mathrm{C} @ @]([\mathrm{H}])(\mathrm{C} / \mathrm{C} 3=\mathrm{C} / \mathrm{C}=\mathrm{CC}=\mathrm{C} 3)[\mathrm{C} @ @](\mathrm{O})([\mathrm{H}]) \mathrm{CN}(\mathrm{CC}(\mathrm{C})(\mathrm{C})[\mathrm{H}])[\mathrm{S}} \\
](=\mathrm{O})(=\mathrm{O}) \mathrm{C} 4=\mathrm{CC}=\mathrm{C}(\mathrm{C}=\mathrm{C} 4) \mathrm{OC}\end{array}$ \\
\hline H112 & + & 3.544 & 3.284 & 67.728 & 3.398 & 52.028 & $\begin{array}{l}\mathrm{O}=\mathrm{C} 1 \mathrm{O}[\mathrm{C} @ @]([\mathrm{H}])(\mathrm{CN} 1 \mathrm{C}=2 \mathrm{C}=\mathrm{CC}=\mathrm{C}(\mathrm{C}=2) \mathrm{OC}([\mathrm{F}])([\mathrm{F}])[\mathrm{F}]) \mathrm{C}(=\mathrm{O}) \mathrm{N} \\
{[\mathrm{C} @ @]([\mathrm{H}])(\mathrm{C} / \mathrm{C} 3=\mathrm{C} / \mathrm{C}=\mathrm{CC}=\mathrm{C} 3)[\mathrm{C} @ @](\mathrm{O})([\mathrm{H}]) \mathrm{CN}(\mathrm{CC}(\mathrm{C})(\mathrm{C})[\mathrm{H}])[\mathrm{S}} \\
](=\mathrm{O})(=\mathrm{O}) \mathrm{C} 4=\mathrm{CC}=\mathrm{C}(\mathrm{C}=\mathrm{C} 4) \mathrm{CO}\end{array}$ \\
\hline H113 & + & 4.824 & 5.152 & 106.209 & 5.224 & 80.029 & $\begin{array}{l}\mathrm{O}=\mathrm{C} 1 \mathrm{O}[\mathrm{C} @ @]([\mathrm{H}])(\mathrm{CN} 1 \mathrm{C}=2 \mathrm{C}=\mathrm{CC}=\mathrm{C}(\mathrm{C}=2) \mathrm{C}(\mathrm{C})=\mathrm{O}) \mathrm{C}(=\mathrm{O}) \mathrm{N}[\mathrm{C} @ @] \\
([\mathrm{H}])(\mathrm{C} / \mathrm{C} 3=\mathrm{C} / \mathrm{C}=\mathrm{CC}=\mathrm{C} 3)[\mathrm{C} @ @](\mathrm{O})([\mathrm{H}]) \mathrm{CN}(\mathrm{CC}(\mathrm{C})(\mathrm{C})[\mathrm{H}])[\mathrm{S}](=\mathrm{O})(= \\
\mathrm{O}) \mathrm{C} 5=\mathrm{CC}=\mathrm{C} 4 \mathrm{~N}=\mathrm{C}[\mathrm{S}] \mathrm{C} 4=\mathrm{C} 5\end{array}$ \\
\hline H115 & + & 4.310 & 4.837 & 99.707 & 4.863 & 74.499 & $\begin{array}{l}\mathrm{O}=\mathrm{C} 1 \mathrm{O}[\mathrm{C} @ @]([\mathrm{H}])(\mathrm{CN} 1 \mathrm{C}=2 \mathrm{C}=\mathrm{CC}=\mathrm{C}(\mathrm{C}=2)[\mathrm{S}](\mathrm{C})(=\mathrm{O})=\mathrm{O}) \mathrm{C}(=\mathrm{O}) \mathrm{N}[ \\
\mathrm{C} @ @]([\mathrm{H}])(\mathrm{C} / \mathrm{C} 3=\mathrm{C} / \mathrm{C}=\mathrm{CC}=\mathrm{C} 3)[\mathrm{C} @ @](\mathrm{O})([\mathrm{H}]) \mathrm{CN}(\mathrm{CC}(\mathrm{C})(\mathrm{C})[\mathrm{H}])[\mathrm{S}] \\
(=\mathrm{O})(=\mathrm{O}) \mathrm{C} 4=\mathrm{CC}=\mathrm{C}(\mathrm{C}=\mathrm{C} 4) \mathrm{OC}\end{array}$ \\
\hline H116 & + & 4.602 & 4.740 & 97.718 & 4.701 & 72.003 & $\begin{array}{l}\mathrm{O}=\mathrm{C} 1 \mathrm{O}[\mathrm{C} @ @]([\mathrm{H}])(\mathrm{CN} 1 \mathrm{C}=2 \mathrm{C}=\mathrm{CC}=\mathrm{C}(\mathrm{C}=2)[\mathrm{S}](\mathrm{C})(=\mathrm{O})=\mathrm{O}) \mathrm{C}(=\mathrm{O}) \mathrm{N}[ \\
\mathrm{C} @ @]([\mathrm{H}])(\mathrm{C} / \mathrm{C} 3=\mathrm{C} / \mathrm{C}=\mathrm{CC}=\mathrm{C} 3)[\mathrm{C} @ @](\mathrm{O})([\mathrm{H}]) \mathrm{CN}(\mathrm{CC}(\mathrm{C})(\mathrm{C})[\mathrm{H}])[\mathrm{S}]\end{array}$ \\
\hline
\end{tabular}




\begin{tabular}{|c|c|c|c|c|c|c|c|}
\hline & & & & & & & $(=0)(=0) C 5=C C=C 4 O C O C 4=C 5$ \\
\hline H118 & + & 3.866 & 4.195 & 86.487 & 4.227 & 64.735 & $\begin{array}{l}\mathrm{O}=\mathrm{C} 10[\mathrm{C} @ @]([\mathrm{H}])(\mathrm{CN} 1 \mathrm{C}=2 \mathrm{C}=\mathrm{CC}=\mathrm{C}(\mathrm{C}=2)[\mathrm{N}+](=\mathrm{O})[\mathrm{O}- \\
]) \mathrm{C}(=\mathrm{O}) \mathrm{N}[\mathrm{C} @ @]([\mathrm{H}])(\mathrm{C} / \mathrm{C} 3=\mathrm{C} / \mathrm{C}=\mathrm{CC}=\mathrm{C} 3)[\mathrm{C} @ @](\mathrm{O})([\mathrm{H}]) \mathrm{CN}(\mathrm{CC}(\mathrm{C} \\
)(\mathrm{C})[\mathrm{H}])[\mathrm{S}](=\mathrm{O})(=\mathrm{O}) \mathrm{C} 4=\mathrm{CC}=\mathrm{C}(\mathrm{C}=\mathrm{C} 4) \mathrm{OC}\end{array}$ \\
\hline H119 & + & 3.932 & 4.098 & 84.498 & 4.064 & 62.240 & $\begin{array}{l}\mathrm{O}=\mathrm{C} 1 \mathrm{O}[\mathrm{C} @ @]([\mathrm{H}])(\mathrm{CN} 1 \mathrm{C}=2 \mathrm{C}=\mathrm{CC}=\mathrm{C}(\mathrm{C}=2)[\mathrm{N}+](=\mathrm{O})[\mathrm{O}- \\
]) \mathrm{C}(=\mathrm{O}) \mathrm{N}[\mathrm{C} @ @]([\mathrm{H}])(\mathrm{C} / \mathrm{C} 3=\mathrm{C} / \mathrm{C}=\mathrm{CC}=\mathrm{C} 3)[\mathrm{C} @ @](\mathrm{O})([\mathrm{H}]) \mathrm{CN}(\mathrm{CC}(\mathrm{C} \\
)(\mathrm{C})[\mathrm{H}])[\mathrm{S}](=\mathrm{O})(=\mathrm{O}) \mathrm{C} 5=\mathrm{CC}=\mathrm{C} 4 \mathrm{OCOC} 4=\mathrm{C} 5\end{array}$ \\
\hline H121 & + & 4.699 & 4.547 & 93.747 & 4.559 & 69.828 & $\begin{array}{l}\mathrm{O}=\mathrm{C} 1 \mathrm{O}[\mathrm{C} @ @]([\mathrm{H}])(\mathrm{CN} 1 \mathrm{C}=2 \mathrm{C}=\mathrm{C} / \mathrm{C}=\mathrm{C}(/ \mathrm{N}) \mathrm{C}=2) \mathrm{C}(=\mathrm{O}) \mathrm{N}[\mathrm{C} @ @]([\mathrm{H}] \\
)(\mathrm{C} / \mathrm{C} 3=\mathrm{C} / \mathrm{C}=\mathrm{CC}=\mathrm{C} 3)[\mathrm{C} @ @](\mathrm{O})([\mathrm{H}]) \mathrm{CN}(\mathrm{CC}(\mathrm{C})(\mathrm{C})[\mathrm{H}])[\mathrm{S}](=\mathrm{O})(=\mathrm{O}) \mathrm{C} \\
4=\mathrm{CC}=\mathrm{C}(\mathrm{C}=\mathrm{C} 4) \mathrm{OC}\end{array}$ \\
\hline H122 & + & 3.947 & 4.451 & 91.758 & 4.396 & 67.333 & $\begin{array}{l}\mathrm{O}=\mathrm{C} 1 \mathrm{O}[\mathrm{C} @ @]([\mathrm{H}])(\mathrm{CN} 1 \mathrm{C}=2 \mathrm{C}=\mathrm{C} / \mathrm{C}=\mathrm{C}(/ \mathrm{N}) \mathrm{C}=2) \mathrm{C}(=\mathrm{O}) \mathrm{N}[\mathrm{C} @ @]([\mathrm{H}] \\
)(\mathrm{C} / \mathrm{C} 3=\mathrm{C} / \mathrm{C}=\mathrm{CC}=\mathrm{C} 3)[\mathrm{C} @ @](\mathrm{O})([\mathrm{H}]) \mathrm{CN}(\mathrm{CC}(\mathrm{C})(\mathrm{C})[\mathrm{H}])[\mathrm{S}](=\mathrm{O})(=\mathrm{O}) \mathrm{C} \\
5=\mathrm{CC}=\mathrm{C} 4 \mathrm{OCOC} 4=\mathrm{C} 5\end{array}$ \\
\hline H123 & + & 5.097 & 4.996 & 102.989 & 4.967 & 76.094 & $\begin{array}{l}\mathrm{O}=\mathrm{C} 10[\mathrm{C} @ @]([\mathrm{H}])(\mathrm{CN} 1 \mathrm{C}=2 \mathrm{C}=\mathrm{C} / \mathrm{C}=\mathrm{C}(/ \mathrm{N}) \mathrm{C}=2) \mathrm{C}(=\mathrm{O}) \mathrm{N}[\mathrm{C} @ @]([\mathrm{H}] \\
)(\mathrm{C} / \mathrm{C} 3=\mathrm{C} / \mathrm{C}=\mathrm{CC}=\mathrm{C} 3)[\mathrm{C} @ @](\mathrm{O})([\mathrm{H}]) \mathrm{CN}(\mathrm{CC}(\mathrm{C})(\mathrm{C})[\mathrm{H}])[\mathrm{S}](=\mathrm{O})(=\mathrm{O}) \mathrm{C} \\
5=\mathrm{CC}=\mathrm{C} 4 \mathrm{~N}=\mathrm{C}[\mathrm{S}] \mathrm{C} 4=\mathrm{C} 5\end{array}$ \\
\hline H126 & + & 5.222 & 4.679 & 96.460 & 4.751 & 72.783 & $\begin{array}{l}\mathrm{O}=\mathrm{C} 1 \mathrm{O}[\mathrm{C} @ @]([\mathrm{H}])(\mathrm{CN} 1 \mathrm{C}=2 \mathrm{C}=\mathrm{CC}=\mathrm{C}(\mathrm{C}=2) \mathrm{NC}(=\mathrm{O}) \mathrm{C}) \mathrm{C}(=\mathrm{O}) \mathrm{N}[\mathrm{C} @ \\
@]([\mathrm{H}])(\mathrm{C} / \mathrm{C} 3=\mathrm{C} / \mathrm{C}=\mathrm{CC}=\mathrm{C} 3)[\mathrm{C} @ @](\mathrm{O})([\mathrm{H}]) \mathrm{CN}(\mathrm{CC}(\mathrm{C})(\mathrm{C})[\mathrm{H}])[\mathrm{S}](=\mathrm{O} \\
)(=\mathrm{O}) \mathrm{C} 5=\mathrm{CC}=\mathrm{C} 4 \mathrm{~N}=\mathrm{C}[\mathrm{S}] \mathrm{C} 4=\mathrm{C} 5\end{array}$ \\
\hline H129 & + & 4.585 & 4.327 & 89.210 & 4.262 & 65.280 & $\begin{array}{l}\mathrm{O}=\mathrm{C} 1 \mathrm{O}[\mathrm{C} @ @]([\mathrm{H}])(\mathrm{CN} 1 \mathrm{C}=2 \mathrm{C}=\mathrm{CC}=\mathrm{C}(\mathrm{C}=2) \mathrm{NC}(=\mathrm{O}) \mathrm{OC}) \mathrm{C}(=\mathrm{O}) \mathrm{N}[\mathrm{C} @ \\
@]([\mathrm{H}])(\mathrm{C} / \mathrm{C} 3=\mathrm{C} / \mathrm{C}=\mathrm{CC}=\mathrm{C} 3)[\mathrm{C} @ @](\mathrm{O})([\mathrm{H}]) \mathrm{CN}(\mathrm{CC}(\mathrm{C})(\mathrm{C})[\mathrm{H}])[\mathrm{S}](=\mathrm{O} \\
)(=\mathrm{O}) \mathrm{C} 5=\mathrm{CC}=\mathrm{C} 4 \mathrm{~N}=\mathrm{C}[\mathrm{S}] \mathrm{C} 4=\mathrm{C} 5\end{array}$ \\
\hline H3 & - & 1.923 & 1.962 & 40.494 & 1.952 & 29.860 & $\begin{array}{l}\mathrm{O}=\mathrm{C}(\mathrm{OCCNC}(=\mathrm{O}) \mathrm{C}([\mathrm{F}])([\mathrm{F}])[\mathrm{F}]) \mathrm{N}[\mathrm{C} @ @]([\mathrm{H}])(\mathrm{C} / \mathrm{C} 1=\mathrm{C} / \mathrm{C}=\mathrm{CC}=\mathrm{C} 1)[ \\
\mathrm{C} @](\mathrm{O})([\mathrm{H}]) \mathrm{CN}(\mathrm{C}[\mathrm{C} @ @](\mathrm{C})([\mathrm{H}]) \mathrm{CC})[\mathrm{S}](=\mathrm{O})(=\mathrm{O}) \mathrm{C} 2=\mathrm{CC}=\mathrm{C}(\mathrm{C}=\mathrm{C} 2) \\
\mathrm{OC}\end{array}$ \\
\hline $\mathrm{H} 7$ & - & 4.553 & 4.521 & 93.208 & 4.623 & 70.810 & 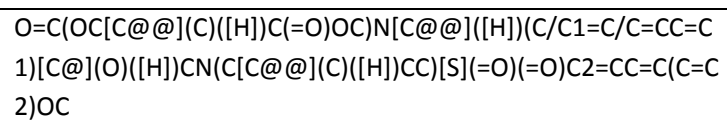 \\
\hline $\mathrm{H} 10$ & - & 1.455 & 1.077 & 22.249 & 1.087 & 16.594 & $\begin{array}{l}\mathrm{O}=\mathrm{C}(\mathrm{O}[\mathrm{C} @] 1([\mathrm{H}]) \mathrm{CCOC1}=\mathrm{O}) \mathrm{N}[\mathrm{C} @ @]([\mathrm{H}])(\mathrm{C} / \mathrm{C} 2=\mathrm{C} / \mathrm{C}=\mathrm{CC}=\mathrm{C} 2)[\mathrm{C} \\
@](\mathrm{O})([\mathrm{H}]) \mathrm{CN}(\mathrm{C}[\mathrm{C} @ @](\mathrm{C})([\mathrm{H}]) \mathrm{CC})[\mathrm{S}](=\mathrm{O})(=\mathrm{O}) \mathrm{C} 3=\mathrm{CC}=\mathrm{C}(\mathrm{C}=\mathrm{C} 3) \mathrm{O} \\
\mathrm{C}\end{array}$ \\
\hline H12 & - & 3.229 & 2.980 & 61.465 & 3.027 & 46.344 & $\begin{array}{l}\mathrm{O}=\mathrm{C}(\mathrm{O}[\mathrm{C} @ @](\mathrm{C})([\mathrm{H}]) \mathrm{CC}(=\mathrm{O}) \mathrm{OC}) \mathrm{N}[\mathrm{C} @ @]([\mathrm{H}])(\mathrm{C} / \mathrm{C} 1=\mathrm{C} / \mathrm{C}=\mathrm{CC}=\mathrm{C} \\
1)[\mathrm{C} @](\mathrm{O})([\mathrm{H}]) \mathrm{CN}(\mathrm{C}[\mathrm{C} @ @](\mathrm{C})([\mathrm{H}]) \mathrm{CC})[\mathrm{S}](=\mathrm{O})(=\mathrm{O}) \mathrm{C} 3=\mathrm{CC}=\mathrm{C} 2 \mathrm{~N}= \\
\mathrm{C}[\mathrm{S}] \mathrm{C} 2=\mathrm{C3}\end{array}$ \\
\hline $\mathrm{H} 20$ & - & 0.915 & 1.149 & 23.744 & 1.170 & 17.868 & $\begin{array}{l}\mathrm{O}=\mathrm{C}(\mathrm{O}[\mathrm{C} @] 1([\mathrm{H}]) \mathrm{CCOC} 1=\mathrm{O}) \mathrm{N}[\mathrm{C} @ @]([\mathrm{H}])(\mathrm{C} / \mathrm{C} 2=\mathrm{C} / \mathrm{C}=\mathrm{CC}=\mathrm{C} 2)[\mathrm{C} \\
@](\mathrm{O})([\mathrm{H}]) \mathrm{CN}(\mathrm{C}[\mathrm{C} @ @](\mathrm{C})([\mathrm{H}]) \mathrm{CC})[\mathrm{S}](=\mathrm{O})(=\mathrm{O}) \mathrm{C} 4=\mathrm{CC}=\mathrm{C} 3 \mathrm{~N}=\mathrm{C}[\mathrm{S}] \\
\mathrm{C} 3=\mathrm{C} 4\end{array}$ \\
\hline $\mathrm{H} 22$ & - & 1.763 & 1.961 & 40.465 & 2.066 & 31.608 & $\begin{array}{l}\mathrm{O}=\mathrm{C}(\mathrm{O}[\mathrm{C} @ @](\mathrm{C})([\mathrm{H}]) \mathrm{CC}(=\mathrm{O}) \mathrm{OC}) \mathrm{N}[\mathrm{C} @ @]([\mathrm{H}])(\mathrm{C} / \mathrm{C} 1=\mathrm{C} / \mathrm{C}=\mathrm{CC}=\mathrm{C} \\
1)[\mathrm{C} @](\mathrm{O})([\mathrm{H}]) \mathrm{CN}(\mathrm{CCCCC})[\mathrm{S}](=\mathrm{O})(=\mathrm{O}) \mathrm{C} 2=\mathrm{CC}=\mathrm{C}(\mathrm{C}=\mathrm{C} 2) \mathrm{OC}\end{array}$ \\
\hline $\mathrm{H} 23$ & - & 1.503 & 1.344 & 27.765 & 1.417 & 21.649 & $\begin{array}{l}\mathrm{O}=\mathrm{C}(\mathrm{OCCNC}(=\mathrm{O}) \mathrm{C}([\mathrm{F}])([\mathrm{F}])[\mathrm{F}]) \mathrm{N}[\mathrm{C} @ @]([\mathrm{H}])(\mathrm{C} / \mathrm{C} 1=\mathrm{C} / \mathrm{C}=\mathrm{CC}=\mathrm{C} 1)[ \\
\mathrm{C} @](\mathrm{O})([\mathrm{H}]) \mathrm{CN}(\mathrm{CCCCC})[\mathrm{S}](=\mathrm{O})(=\mathrm{O}) \mathrm{C} 2=\mathrm{CC}=\mathrm{C}(\mathrm{C}=\mathrm{C} 2) \mathrm{OC}\end{array}$ \\
\hline $\mathrm{H} 43$ & - & 4.180 & 4.074 & 83.984 & 4.083 & 62.529 & $\begin{array}{l}\mathrm{O}=\mathrm{C} 1 \mathrm{O}[\mathrm{C} @ @]([\mathrm{H}])(\mathrm{CN} 1 \mathrm{C}=2 \mathrm{C}=\mathrm{CC}([\mathrm{F}])=\mathrm{C}([\mathrm{F}]) \mathrm{C}=2) \mathrm{C}(=\mathrm{O}) \mathrm{N}[\mathrm{C} @ @] \\
([\mathrm{H}])(\mathrm{C} / \mathrm{C} 3=\mathrm{C} / \mathrm{C}=\mathrm{CC}=\mathrm{C} 3)[\mathrm{C} @ @](\mathrm{O})([\mathrm{H}]) \mathrm{CN}(\mathrm{CC}(\mathrm{C})(\mathrm{C})[\mathrm{H}])[\mathrm{S}](=\mathrm{O})(= \\
\mathrm{O}) \mathrm{C} 4=\mathrm{CC}=\mathrm{C}(\mathrm{C}=\mathrm{C} 4) \mathrm{OC}\end{array}$ \\
\hline $\mathrm{H} 47$ & - & 4.347 & 4.704 & 96.981 & 4.637 & 71.029 & $\begin{array}{l}\mathrm{O}=\mathrm{C} 10[\mathrm{C} @ @]([\mathrm{H}])(\mathrm{CN} 1 \mathrm{C}=2 \mathrm{C}=\mathrm{CC}=\mathrm{C}(\mathrm{C}=2) \mathrm{OC}) \mathrm{C}(=\mathrm{O}) \mathrm{N}[\mathrm{C} @ @]([\mathrm{H}] \\
)(\mathrm{C} / \mathrm{C} 3=\mathrm{C} / \mathrm{C}=\mathrm{CC}=\mathrm{C} 3)[\mathrm{C} @ @](\mathrm{O})([\mathrm{H}]) \mathrm{CN}(\mathrm{CC}(\mathrm{C})(\mathrm{C})[\mathrm{H}])[\mathrm{S}](=\mathrm{O})(=\mathrm{O}) \mathrm{C}\end{array}$ \\
\hline
\end{tabular}




\begin{tabular}{|c|c|c|c|c|c|c|c|}
\hline & & & & & & & $4=C C=C(C=C 4) O C$ \\
\hline H55 & - & 4.071 & 3.977 & 81.995 & 3.920 & 60.034 & $\begin{array}{l}\mathrm{O}=\mathrm{C} 1 \mathrm{O}[\mathrm{C} @ @]([\mathrm{H}])(\mathrm{CN} 1 \mathrm{C}=2 \mathrm{C}=\mathrm{CC}([\mathrm{F}])=\mathrm{C}([\mathrm{F}]) \mathrm{C}=2) \mathrm{C}(=\mathrm{O}) \mathrm{N}[\mathrm{C} @ @] \\
([\mathrm{H}])(\mathrm{C} / \mathrm{C} 3=\mathrm{C} / \mathrm{C}=\mathrm{CC}=\mathrm{C} 3)[\mathrm{C} @ @](\mathrm{O})([\mathrm{H}]) \mathrm{CN}(\mathrm{CC}(\mathrm{C})(\mathrm{C})[\mathrm{H}])[\mathrm{S}](=\mathrm{O})(= \\
\mathrm{O}) \mathrm{C} 5=\mathrm{CC}=\mathrm{C} 4 \mathrm{OCOC} 4=\mathrm{C} 5\end{array}$ \\
\hline H59 & - & 4.155 & 3.938 & 81.183 & 3.956 & 60.585 & $\begin{array}{l}\mathrm{O}=\mathrm{C} 1 \mathrm{O}[\mathrm{C} @ @]([\mathrm{H}])(\mathrm{CN} 1 \mathrm{C}=2 \mathrm{C}=\mathrm{C} / \mathrm{C}=\mathrm{C}(/[\mathrm{F}]) \mathrm{C}=2) \mathrm{C}(=\mathrm{O}) \mathrm{N}[\mathrm{C} @ @]([ \\
\mathrm{H}])(\mathrm{C} / \mathrm{C} 3=\mathrm{C} / \mathrm{C}=\mathrm{CC}=\mathrm{C} 3)[\mathrm{C} @ @](\mathrm{O})([\mathrm{H}]) \mathrm{CN}(\mathrm{CC}(\mathrm{C})(\mathrm{C})[\mathrm{H}])[\mathrm{S}](=\mathrm{O})(=\mathrm{O} \\
) \mathrm{C} 4=\mathrm{C} / \mathrm{C}=\mathrm{C}(/ \mathrm{OC}) \mathrm{C}(\mathrm{C})=\mathrm{C} 4\end{array}$ \\
\hline H64 & - & 2.000 & 2.193 & 45.249 & 2.142 & 32.769 & $\begin{array}{l}\mathrm{O}=\mathrm{C} 1 \mathrm{O}[\mathrm{C} @ @]([\mathrm{H}])(\mathrm{CN} 1 \mathrm{C}=2 \mathrm{C}=\mathrm{CC}=\mathrm{C}(\mathrm{C}=2) \mathrm{C}([\mathrm{F}])([\mathrm{F}])[\mathrm{F}]) \mathrm{C}(=\mathrm{O}) \mathrm{N}[\mathrm{C} \\
@ @]([\mathrm{H}])(\mathrm{C} / \mathrm{C} 3=\mathrm{C} / \mathrm{C}=\mathrm{CC}=\mathrm{C} 3)[\mathrm{C} @ @](\mathrm{O})([\mathrm{H}]) \mathrm{CN}(\mathrm{CC}(\mathrm{C})(\mathrm{C})[\mathrm{H}])[\mathrm{S}]( \\
=\mathrm{O})(=\mathrm{O}) \mathrm{C} 4=\mathrm{CC}=\mathrm{C}(\mathrm{C}=\mathrm{C} 4) \mathrm{OC}([\mathrm{F}])([\mathrm{F}])[\mathrm{F}]^{\sim}\end{array}$ \\
\hline H65 & - & 2.699 & 3.356 & 69.211 & 3.364 & 51.501 & $\begin{array}{l}\mathrm{O}=\mathrm{C} 1 \mathrm{O}[\mathrm{C} @ @]([\mathrm{H}])(\mathrm{CN} 1 \mathrm{C}=2 \mathrm{C}=\mathrm{CC}=\mathrm{C}(\mathrm{C}=2) \mathrm{C}(=\mathrm{O}) \mathrm{C}) \mathrm{C}(=\mathrm{O}) \mathrm{N}[\mathrm{C} @ @] \\
([\mathrm{H}])(\mathrm{C} / \mathrm{C} 3=\mathrm{C} / \mathrm{C}=\mathrm{CC}=\mathrm{C} 3)[\mathrm{C} @ @](\mathrm{O})([\mathrm{H}]) \mathrm{CN}(\mathrm{CC}(\mathrm{C})(\mathrm{C})[\mathrm{H}])[\mathrm{S}](=\mathrm{O})(= \\
\mathrm{O}) \mathrm{C} 4=\mathrm{CC}=\mathrm{C}(\mathrm{C}=\mathrm{C} 4) \mathrm{OC}([\mathrm{F}])([\mathrm{F}])[\mathrm{F}]\end{array}$ \\
\hline H107 & - & 3.708 & 3.382 & 69.731 & 3.235 & 49.532 & $\begin{array}{l}\mathrm{O}=\mathrm{C} 1 \mathrm{O}[\mathrm{C} @ @]([\mathrm{H}])(\mathrm{CN} 1 \mathrm{C}=2 \mathrm{C}=\mathrm{CC}=\mathrm{C}(\mathrm{C}=2) \mathrm{C}([\mathrm{F}])([\mathrm{F}])[\mathrm{F}]) \mathrm{C}(=\mathrm{O}) \mathrm{N}[\mathrm{C} \\
@ @]([\mathrm{H}])(\mathrm{C} / \mathrm{C} 3=\mathrm{C} / \mathrm{C}=\mathrm{CC}=\mathrm{C} 3)[\mathrm{C} @ @](\mathrm{O})([\mathrm{H}]) \mathrm{CN}(\mathrm{CC}(\mathrm{C})(\mathrm{C})[\mathrm{H}])[\mathrm{S}]( \\
=\mathrm{O})(=\mathrm{O}) \mathrm{C} 4=\mathrm{CC}=\mathrm{C}(\mathrm{C}=\mathrm{C} 4) \mathrm{CO}\end{array}$ \\
\hline H111 & - & 4.585 & 4.231 & 87.231 & 4.328 & 66.285 & $\begin{array}{l}\mathrm{O}=\mathrm{C} 1 \mathrm{O}[\mathrm{C} @ @]([\mathrm{H}])(\mathrm{CN} 1 \mathrm{C}=2 \mathrm{C}=\mathrm{CC}=\mathrm{C}(\mathrm{C}=2) \mathrm{OC}([\mathrm{F}])([\mathrm{F}])[\mathrm{F}]) \mathrm{C}(=\mathrm{O}) \mathrm{N} \\
{[\mathrm{C} @ @]([\mathrm{H}])(\mathrm{C} / \mathrm{C} 3=\mathrm{C} / \mathrm{C}=\mathrm{CC}=\mathrm{C} 3)[\mathrm{C} @ @](\mathrm{O})([\mathrm{H}]) \mathrm{CN}(\mathrm{CC}(\mathrm{C})(\mathrm{C})[\mathrm{H}])[\mathrm{S}} \\
](=\mathrm{O})(=\mathrm{O}) \mathrm{C} 5=\mathrm{CC}=\mathrm{C} 4 \mathrm{~N}=\mathrm{C}[\mathrm{S}] \mathrm{C} 4=\mathrm{C} 5\end{array}$ \\
\hline H114 & - & 3.627 & 4.206 & 86.706 & 4.294 & 65.772 & $\begin{array}{l}\mathrm{O}=\mathrm{C} 1 \mathrm{O}[\mathrm{C} @ @]([\mathrm{H}])(\mathrm{CN} 1 \mathrm{C}=2 \mathrm{C}=\mathrm{CC}=\mathrm{C}(\mathrm{C}=2) \mathrm{C}(\mathrm{C})=\mathrm{O}) \mathrm{C}(=\mathrm{O}) \mathrm{N}[\mathrm{C} @ @] \\
([\mathrm{H}])(\mathrm{C} / \mathrm{C} 3=\mathrm{C} / \mathrm{C}=\mathrm{CC}=\mathrm{C} 3)[\mathrm{C} @ @](\mathrm{O})([\mathrm{H}]) \mathrm{CN}(\mathrm{CC}(\mathrm{C})(\mathrm{C})[\mathrm{H}])[\mathrm{S}](=\mathrm{O})(= \\
\mathrm{O}) \mathrm{C} 4=\mathrm{CC}=\mathrm{C}(\mathrm{C}=\mathrm{C} 4) \mathrm{CO}\end{array}$ \\
\hline H117 & - & 5.523 & 5.285 & 108.949 & 5.272 & 80.765 & $\begin{array}{l}\mathrm{O}=\mathrm{C} 1 \mathrm{O}[\mathrm{C} @ @]([\mathrm{H}])(\mathrm{CN} 1 \mathrm{C}=2 \mathrm{C}=\mathrm{CC}=\mathrm{C}(\mathrm{C}=2)[\mathrm{S}](\mathrm{C})(=\mathrm{O})=\mathrm{O}) \mathrm{C}(=\mathrm{O}) \mathrm{N}[ \\
\mathrm{C} @ @]([\mathrm{H}])(\mathrm{C} / \mathrm{C} 3=\mathrm{C} / \mathrm{C}=\mathrm{CC}=\mathrm{C} 3)[\mathrm{C} @ @](\mathrm{O})([\mathrm{H}]) \mathrm{CN}(\mathrm{CC}(\mathrm{C})(\mathrm{C})[\mathrm{H}])[\mathrm{S}] \\
(=\mathrm{O})(=\mathrm{O}) \mathrm{C} 5=\mathrm{CC}=\mathrm{C} 4 \mathrm{~N}=\mathrm{C}[\mathrm{S}] \mathrm{C} 4=\mathrm{C} 5\end{array}$ \\
\hline H120 & - & 4.824 & 4.644 & 95.729 & 4.635 & 71.001 & 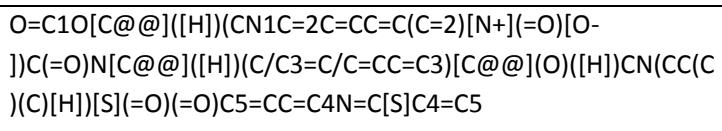 \\
\hline H124 & - & 3.914 & 4.231 & 87.219 & 4.343 & 66.517 & $\begin{array}{l}\mathrm{O}=\mathrm{C} 1 \mathrm{O}[\mathrm{C} @ @]([\mathrm{H}])(\mathrm{CN} 1 \mathrm{C}=2 \mathrm{C}=\mathrm{CC}=\mathrm{C}(\mathrm{C}=2) \mathrm{NC}(=\mathrm{O}) \mathrm{C}) \mathrm{C}(=\mathrm{O}) \mathrm{N}[\mathrm{C} @ \\
@]([\mathrm{H}])(\mathrm{C} / \mathrm{C} 3=\mathrm{C} / \mathrm{C}=\mathrm{CC}=\mathrm{C} 3)[\mathrm{C} @ @](\mathrm{O})([\mathrm{H}]) \mathrm{CN}(\mathrm{CC}(\mathrm{C})(\mathrm{C})[\mathrm{H}])[\mathrm{S}](=\mathrm{O} \\
)(=\mathrm{O}) \mathrm{C} 4=\mathrm{CC}=\mathrm{C}(\mathrm{C}=\mathrm{C} 4) \mathrm{OC}\end{array}$ \\
\hline H127 & - & 3.788 & 3.879 & 79.968 & 3.853 & 59.014 & $\begin{array}{l}\mathrm{O}=\mathrm{C} 1 \mathrm{O}[\mathrm{C} @ @]([\mathrm{H}])(\mathrm{CN} 1 \mathrm{C}=2 \mathrm{C}=\mathrm{CC}=\mathrm{C}(\mathrm{C}=2) \mathrm{NC}(=\mathrm{O}) \mathrm{OC}) \mathrm{C}(=\mathrm{O}) \mathrm{N}[\mathrm{C} @ \\
@]([\mathrm{H}])(\mathrm{C} / \mathrm{C} 3=\mathrm{C} / \mathrm{C}=\mathrm{CC}=\mathrm{C} 3)[\mathrm{C} @ @](\mathrm{O})([\mathrm{H}]) \mathrm{CN}(\mathrm{CC}(\mathrm{C})(\mathrm{C})[\mathrm{H}])[\mathrm{S}](=\mathrm{O} \\
)(=\mathrm{O}) \mathrm{C} 4=\mathrm{CC}=\mathrm{C}(\mathrm{C}=\mathrm{C} 4) \mathrm{OC}\end{array}$ \\
\hline $\mathrm{H} 2$ & $\#$ & 3.059 & 2.579 & 53.194 & 2.602 & 39.819 & 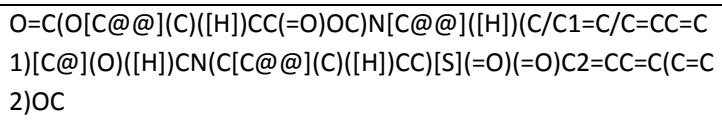 \\
\hline H18 & $\#$ & 5.301 & 4.220 & 87.007 & 4.221 & 64.649 & $\begin{array}{l}\mathrm{O}=\mathrm{C}(\mathrm{OCCN} 1 \mathrm{CCNC1}=\mathrm{O}) \mathrm{N}[\mathrm{C} @ @]([\mathrm{H}])(\mathrm{C} / \mathrm{C} 2=\mathrm{C} / \mathrm{C}=\mathrm{CC}=\mathrm{C} 2)[\mathrm{C} @](\mathrm{O}) \\
([\mathrm{H}]) \mathrm{CN}(\mathrm{C}[\mathrm{C} @ @](\mathrm{C})([\mathrm{H}]) \mathrm{CC})[\mathrm{S}](=\mathrm{O})(=\mathrm{O}) \mathrm{C} 4=\mathrm{CC}=\mathrm{C} 3 \mathrm{~N}=\mathrm{C}[\mathrm{S}] \mathrm{C} 3=\mathrm{C} 4\end{array}$ \\
\hline $\mathrm{H} 26$ & $\#$ & 3.391 & 3.709 & 76.484 & 3.143 & 48.111 & $\begin{array}{l}\mathrm{O}=\mathrm{C}(\mathrm{OC}[\mathrm{C} @](\mathrm{C})([\mathrm{H}]) \mathrm{C}(=\mathrm{O}) \mathrm{OC}) \mathrm{N}[\mathrm{C} @ @]([\mathrm{H}])(\mathrm{C} / \mathrm{C} 1=\mathrm{C} / \mathrm{C}=\mathrm{CC}=\mathrm{C} 1)[ \\
\mathrm{C} @](\mathrm{O})([\mathrm{H}]) \mathrm{CN}(\mathrm{CCCCC})[\mathrm{S}](=\mathrm{O})(=\mathrm{O}) \mathrm{C} 2=\mathrm{CC}=\mathrm{C}(\mathrm{C}=\mathrm{C} 2) \mathrm{OC}\end{array}$ \\
\hline $\mathrm{H} 29$ & $\#$ & 1.181 & 0.459 & 9.520 & 0.552 & 8.383 & $\begin{array}{l}\mathrm{O}=\mathrm{C}(\mathrm{O}[\mathrm{C} @] 1([\mathrm{H}]) \mathrm{CCOC} 1=\mathrm{O}) \mathrm{N}[\mathrm{C} @ @]([\mathrm{H}])(\mathrm{C} / \mathrm{C} 2=\mathrm{C} / \mathrm{C}=\mathrm{CC}=\mathrm{C} 2)[\mathrm{C} \\
@](\mathrm{O})([\mathrm{H}]) \mathrm{CN}(\mathrm{CCCCC})[\mathrm{S}](=\mathrm{O})(=\mathrm{O}) \mathrm{C} 3=\mathrm{CC}=\mathrm{C}(\mathrm{C}=\mathrm{C} 3) \mathrm{OC}\end{array}$ \\
\hline H34 & \# & 2.472 & 2.787 & 57.477 & 2.459 & 37.627 & $\begin{array}{l}\mathrm{O}=\mathrm{C}(\mathrm{O}[\mathrm{C} @ @](\mathrm{C})([\mathrm{H}]) \mathrm{CCC}(\mathrm{C})=\mathrm{O}) \mathrm{N}[\mathrm{C} @ @]([\mathrm{H}])(\mathrm{C} / \mathrm{C} 1=\mathrm{C} / \mathrm{C}=\mathrm{CC}=\mathrm{C} 1 \\
)[\mathrm{C} @](\mathrm{O})([\mathrm{H}]) \mathrm{CN}(\mathrm{CCCCC})[\mathrm{S}](=\mathrm{O})(=\mathrm{O}) \mathrm{C} 3=\mathrm{CC}=\mathrm{C} 2 \mathrm{~N}=\mathrm{C}[\mathrm{S}] \mathrm{C} 2=\mathrm{C} 3\end{array}$ \\
\hline
\end{tabular}




\begin{tabular}{|c|c|c|c|c|c|c|c|}
\hline H36 & \# & 2.476 & 4.111 & 84.755 & 3.568 & 54.636 & $\begin{array}{l}\mathrm{O}=\mathrm{C}(\mathrm{OC}[\mathrm{C} @](\mathrm{C})([\mathrm{H}]) \mathrm{C}(=\mathrm{O}) \mathrm{OC}) \mathrm{N}[\mathrm{C} @ @]([\mathrm{H}])(\mathrm{C} / \mathrm{C} 1=\mathrm{C} / \mathrm{C}=\mathrm{CC}=\mathrm{C} 1)[ \\
\mathrm{C} @](\mathrm{O})([\mathrm{H}]) \mathrm{CN}(\mathrm{CCCCC})[\mathrm{S}](=\mathrm{O})(=\mathrm{O}) \mathrm{C} 3=\mathrm{CC}=\mathrm{C} 2 \mathrm{~N}=\mathrm{C}[\mathrm{S}] \mathrm{C} 2=\mathrm{C} 3\end{array}$ \\
\hline $\mathrm{H} 40$ & \# & 4.569 & 4.111 & 84.747 & 4.123 & 63.154 & $\begin{array}{l}\mathrm{O}=\mathrm{C}(\mathrm{OC}[\mathrm{C} @] 1([\mathrm{H}]) \mathrm{NC}(=\mathrm{O}) \mathrm{CC} 1) \mathrm{N}[\mathrm{C} @ @]([\mathrm{H}])(\mathrm{C} / \mathrm{C} 2=\mathrm{C} / \mathrm{C}=\mathrm{CC}=\mathrm{C} 2) \\
{[\mathrm{C} @](\mathrm{O})([\mathrm{H}]) \mathrm{CN}(\mathrm{CCCCC})[\mathrm{S}](=\mathrm{O})(=\mathrm{O}) \mathrm{C} 4=\mathrm{CC}=\mathrm{C} 3 \mathrm{~N}=\mathrm{C}[\mathrm{S}] \mathrm{C} 3=\mathrm{C} 4}\end{array}$ \\
\hline H44 & \# & 5.222 & 3.880 & 79.993 & 3.756 & 57.523 & $\begin{array}{l}\mathrm{O}=\mathrm{C} 1 \mathrm{O}[\mathrm{C} @ @]([\mathrm{H}])(\mathrm{CN} 1 \mathrm{C}=2 \mathrm{C}=\mathrm{CC}=\mathrm{C}(\mathrm{C}=2) \mathrm{C}([\mathrm{F}])([\mathrm{F}])[\mathrm{F}]) \mathrm{C}(=\mathrm{O}) \mathrm{N}[\mathrm{C} \\
@ @]([\mathrm{H}])(\mathrm{C} / \mathrm{C} 3=\mathrm{C} / \mathrm{C}=\mathrm{CC}=\mathrm{C} 3)[\mathrm{C} @ @](\mathrm{O})([\mathrm{H}]) \mathrm{CN}(\mathrm{CC}(\mathrm{C})(\mathrm{C})[\mathrm{H}])[\mathrm{S}]( \\
=0)(=\mathrm{O}) \mathrm{C} 4=\mathrm{CC}=\mathrm{C}(\mathrm{C}=\mathrm{C} 4) \mathrm{OC}\end{array}$ \\
\hline H50 & $\#$ & 3.638 & 3.709 & 76.480 & 3.662 & 56.077 & $\begin{array}{l}\mathrm{O}=\mathrm{C} 10[\mathrm{C} @ @]([\mathrm{H}])(\mathrm{CN} 1 \mathrm{C}=2 \mathrm{C}=\mathrm{CC}([\mathrm{F}])=\mathrm{C}([\mathrm{F}]) \mathrm{C}=2) \mathrm{C}(=\mathrm{O}) \mathrm{N}[\mathrm{C} @ @] \\
([\mathrm{H}])(\mathrm{C} / \mathrm{C} 3=\mathrm{C} / \mathrm{C}=\mathrm{CC}=\mathrm{C} 3)[\mathrm{C} @ @](\mathrm{O})([\mathrm{H}]) \mathrm{CN}(\mathrm{CC}(\mathrm{C})(\mathrm{C})[\mathrm{H}])[\mathrm{S}](=\mathrm{O})(= \\
\mathrm{O}) \mathrm{C} 4=\mathrm{C} / \mathrm{C}=\mathrm{C}(/ \mathrm{N}) \mathrm{C}=\mathrm{C} 4\end{array}$ \\
\hline H58 & \# & 4.796 & 4.036 & 83.217 & 3.903 & 59.768 & $\begin{array}{l}\mathrm{O}=\mathrm{C} 1 \mathrm{O}[\mathrm{C} @ @]([\mathrm{H}])(\mathrm{CN} 1 \mathrm{C}=2 \mathrm{C}=\mathrm{CC}(=\mathrm{CC}=2) \mathrm{C}(=\mathrm{O}) \mathrm{C}) \mathrm{C}(=\mathrm{O}) \mathrm{N}[\mathrm{C} @ @] \\
([\mathrm{H}])(\mathrm{C} / \mathrm{C} 3=\mathrm{C} / \mathrm{C}=\mathrm{CC}=\mathrm{C} 3)[\mathrm{C} @ @](\mathrm{O})([\mathrm{H}]) \mathrm{CN}(\mathrm{CC}(\mathrm{C})(\mathrm{C})[\mathrm{H}])[\mathrm{S}](=\mathrm{O})(= \\
\mathrm{O}) \mathrm{C} 5=\mathrm{CC}=\mathrm{C} 4 \mathrm{OCOC} 4=\mathrm{C} 5\end{array}$ \\
\hline H61 & $\#$ & 4.143 & 3.769 & 77.702 & 3.627 & 55.541 & $\begin{array}{l}\mathrm{O}=\mathrm{C} 10[\mathrm{C} @ @]([\mathrm{H}])(\mathrm{CN} 1 \mathrm{C}=2 \mathrm{C}=\mathrm{CC}=\mathrm{C}(\mathrm{C}=2) \mathrm{C}([\mathrm{F}])([\mathrm{F}])[\mathrm{F}]) \mathrm{C}(=\mathrm{O}) \mathrm{N}[\mathrm{C} \\
@ @]([\mathrm{H}])(\mathrm{C} / \mathrm{C} 3=\mathrm{C} / \mathrm{C}=\mathrm{CC}=\mathrm{C} 3)[\mathrm{C} @ @](\mathrm{O})([\mathrm{H}]) \mathrm{CN}(\mathrm{CC}(\mathrm{C})(\mathrm{C})[\mathrm{H}])[\mathrm{S}]( \\
=0)(=\mathrm{O}) \mathrm{C} 4=\mathrm{C} / \mathrm{C}=\mathrm{C}(/ \mathrm{OC}) \mathrm{C}(\mathrm{C})=\mathrm{C} 4\end{array}$ \\
\hline $\mathrm{H} 70$ & $\#$ & 3.097 & 3.491 & 71.985 & 3.413 & 52.264 & $\begin{array}{l}\mathrm{O}=\mathrm{C} 10[\mathrm{C} @ @]([\mathrm{H}])(\mathrm{CN} 1 \mathrm{C}=2 \mathrm{C}=\mathrm{CC}(=\mathrm{CC}=2) \mathrm{C}(\mathrm{C})=\mathrm{O}) \mathrm{C}(=\mathrm{O}) \mathrm{N}[\mathrm{C} @ @] \\
([\mathrm{H}])(\mathrm{C} / \mathrm{C} 3=\mathrm{C} / \mathrm{C}=\mathrm{CC}=\mathrm{C} 3)[\mathrm{C} @ @](\mathrm{O})([\mathrm{H}]) \mathrm{CN}(\mathrm{C}[\mathrm{C} @ @] 4([\mathrm{H}]) \mathrm{CC} 4)[\mathrm{S} \\
](=\mathrm{S})(=\mathrm{O}) \mathrm{C} 5=\mathrm{CC}=\mathrm{C}(\mathrm{C}=\mathrm{C} 5) \mathrm{OC}\end{array}$ \\
\hline H74 & \# & 0.769 & 0.580 & 12.021 & 0.844 & 12.868 & $\begin{array}{l}\mathrm{O}=\mathrm{C} 10[\mathrm{C} @ @]([\mathrm{H}])(\mathrm{CN} 1 \mathrm{C}=2 \mathrm{C}=\mathrm{CC}=\mathrm{CC}=2) \mathrm{C}(=\mathrm{O}) \mathrm{N}[\mathrm{C} @ @]([\mathrm{H}])(\mathrm{C} / \\
\mathrm{C} 3=\mathrm{C} / \mathrm{C}=\mathrm{CC}=\mathrm{C} 3)[\mathrm{C} @ @](\mathrm{O})([\mathrm{H}]) \mathrm{CN}(\mathrm{C} / \mathrm{C} 4=\mathrm{C} / \mathrm{C}=\mathrm{C}[\mathrm{S}] 4)[\mathrm{S}](=\mathrm{O})(=\mathrm{O}) \\
\mathrm{C} 5=\mathrm{CC}([\mathrm{F}])=\mathrm{C}([\mathrm{F}]) \mathrm{C}=\mathrm{C} 5[\mathrm{~F}]\end{array}$ \\
\hline H75 & $\#$ & 0.795 & 0.434 & 9.006 & 0.749 & 11.403 & $\begin{array}{l}\mathrm{O}=\mathrm{C} 1 \mathrm{O}[\mathrm{C} @ @]([\mathrm{H}])(\mathrm{CN} 1 \mathrm{C}=2 \mathrm{C}=\mathrm{C} / \mathrm{C}=\mathrm{C}(/[\mathrm{F}]) \mathrm{C}=2) \mathrm{C}(=\mathrm{O}) \mathrm{N}[\mathrm{C} @ @]([ \\
\mathrm{H}])(\mathrm{C} / \mathrm{C} 3=\mathrm{C} / \mathrm{C}=\mathrm{CC}=\mathrm{C} 3)[\mathrm{C} @ @](\mathrm{O})([\mathrm{H}]) \mathrm{CN}(\mathrm{C} / \mathrm{C} 4=\mathrm{C} / \mathrm{C}=\mathrm{C}[\mathrm{S}] 4)[\mathrm{S}](= \\
\mathrm{O})(=\mathrm{O}) \mathrm{C} 5=\mathrm{CC}([\mathrm{F}])=\mathrm{C}([\mathrm{F}]) \mathrm{C}=\mathrm{C} 5[\mathrm{~F}]\end{array}$ \\
\hline H82 & \# & 2.133 & 1.914 & 39.500 & 1.886 & 28.835 & $\begin{array}{l}\mathrm{O}=\mathrm{C} 1 \mathrm{O}[\mathrm{C} @ @]([\mathrm{H}])(\mathrm{CN} 1 \mathrm{C}=2 \mathrm{C}=\mathrm{CC}=\mathrm{CC}=2 \mathrm{O}) \mathrm{C}(=\mathrm{O}) \mathrm{N}[\mathrm{C} @ @]([\mathrm{H}])(\mathrm{C} \\
/ \mathrm{C} 3=\mathrm{C} / \mathrm{C}=\mathrm{CC}=\mathrm{C} 3)[\mathrm{C} @ @](\mathrm{O})([\mathrm{H}]) \mathrm{CN}(\mathrm{CC}(\mathrm{C})(\mathrm{C})[\mathrm{H}])[\mathrm{S}](=\mathrm{O})(=\mathrm{O}) \mathrm{C} 4= \\
\mathrm{C} / \mathrm{C}=\mathrm{C}(/ \mathrm{N}) \mathrm{C}=\mathrm{C} 4\end{array}$ \\
\hline H90 & \# & 3.415 & 3.430 & 70.730 & 3.385 & 51.827 & $\begin{array}{l}\mathrm{O}=\mathrm{C} 1 \mathrm{O}[\mathrm{C} @ @]([\mathrm{H}])(\mathrm{CN} 1 \mathrm{C}=2 \mathrm{C}=\mathrm{CC}([\mathrm{F}])=\mathrm{CC}=2[\mathrm{~F}]) \mathrm{C}(=\mathrm{O}) \mathrm{N}[\mathrm{C} @ @]([ \\
\mathrm{H}])(\mathrm{C} / \mathrm{C} 3=\mathrm{C} / \mathrm{C}=\mathrm{CC}=\mathrm{C} 3)[\mathrm{C} @ @](\mathrm{O})([\mathrm{H}]) \mathrm{CN}(\mathrm{CC}(\mathrm{C})(\mathrm{C})[\mathrm{H}])[\mathrm{S}](=\mathrm{O})(=\mathrm{O} \\
\mathrm{C} 4=\mathrm{C} / \mathrm{C}=\mathrm{C}(/ \mathrm{N}) \mathrm{C}=\mathrm{C} 4\end{array}$ \\
\hline H94 & \# & 3.674 & 3.296 & 67.973 & 3.285 & 50.288 & $\begin{array}{l}\mathrm{O}=\mathrm{C} 1 \mathrm{O}[\mathrm{C} @ @]([\mathrm{H}])(\mathrm{CN} 1 \mathrm{C}=2 \mathrm{C}=\mathrm{CC}([\mathrm{F}])=\mathrm{CC}=2[\mathrm{~F}]) \mathrm{C}(=\mathrm{O}) \mathrm{N}[\mathrm{C} @ @]([ \\
\mathrm{H}])(\mathrm{C} / \mathrm{C} 3=\mathrm{C} / \mathrm{C}=\mathrm{CC}=\mathrm{C} 3)[\mathrm{C} @ @](\mathrm{O})([\mathrm{H}]) \mathrm{CN}(\mathrm{CC}(\mathrm{C})(\mathrm{C})[\mathrm{H}])[\mathrm{S}](=\mathrm{O})(=\mathrm{O} \\
) \mathrm{C} 4=\mathrm{CC}=\mathrm{C}(\mathrm{C}=\mathrm{C} 4) \mathrm{CO}\end{array}$ \\
\hline H96 & \# & 3.893 & 3.770 & 77.732 & 3.904 & 59.784 & $\begin{array}{l}\mathrm{O}=\mathrm{C} 10[\mathrm{C} @ @]([\mathrm{H}])(\mathrm{CN} 1 \mathrm{C}=2 \mathrm{C}=\mathrm{CC}([\mathrm{F}])=\mathrm{CC}=2) \mathrm{C}(=\mathrm{O}) \mathrm{N}[\mathrm{C} @ @]([\mathrm{H}]) \\
(\mathrm{C} / \mathrm{C} 3=\mathrm{C} / \mathrm{C}=\mathrm{CC}=\mathrm{C} 3)[\mathrm{C} @ @](\mathrm{O})([\mathrm{H}]) \mathrm{CN}(\mathrm{CC}(\mathrm{C})(\mathrm{C})[\mathrm{H}])[\mathrm{S}](=\mathrm{O})(=\mathrm{O}) \mathrm{C} \\
4=\mathrm{CC}=\mathrm{C}(\mathrm{C}=\mathrm{C} 4) \mathrm{OC}\end{array}$ \\
\hline H99 & \# & 3.684 & 3.272 & 67.470 & 3.383 & 51.793 & $\begin{array}{l}\mathrm{O}=\mathrm{C} 10[\mathrm{C} @ @]([\mathrm{H}])(\mathrm{CN} 1 \mathrm{C}=2 \mathrm{C}=\mathrm{CC}([\mathrm{F}])=\mathrm{CC}=2) \mathrm{C}(=\mathrm{O}) \mathrm{N}[\mathrm{C} @ @]([\mathrm{H}]) \\
(\mathrm{C} / \mathrm{C} 3=\mathrm{C} / \mathrm{C}=\mathrm{CC}=\mathrm{C} 3)[\mathrm{C} @ @](\mathrm{O})([\mathrm{H}]) \mathrm{CN}(\mathrm{CC}(\mathrm{C})(\mathrm{C})[\mathrm{H}])[\mathrm{S}](=\mathrm{O})(=\mathrm{O}) \mathrm{C} \\
4=\mathrm{CC}=\mathrm{C}(\mathrm{C}=\mathrm{C} 4) \mathrm{CO}\end{array}$ \\
\hline H5 & $*$ & 3.371 & 3.003 & 61.935 & 2.569 & 39.313 & $\begin{array}{l}\mathrm{O}=\mathrm{C}(\mathrm{O}[\mathrm{C} @ @](\mathrm{C})([\mathrm{H}]) \mathrm{CCC}(\mathrm{C})=\mathrm{O}) \mathrm{N}[\mathrm{C} @ @]([\mathrm{H}])(\mathrm{C} / \mathrm{C} 1=\mathrm{C} / \mathrm{C}=\mathrm{CC}=\mathrm{C} 1 \\
)[\mathrm{C} @](\mathrm{O})([\mathrm{H}]) \mathrm{CN}(\mathrm{C}[\mathrm{C} @ @](\mathrm{C})([\mathrm{H}]) \mathrm{CC})[\mathrm{S}](=\mathrm{O})(=\mathrm{O}) \mathrm{C} 2=\mathrm{CC}=\mathrm{C}(\mathrm{C}=\mathrm{C} 2 \\
\text { JOC }\end{array}$ \\
\hline H15 & $*$ & 3.519 & 3.405 & 70.206 & 2.994 & 45.838 & $\begin{array}{l}\mathrm{O}=\mathrm{C}(\mathrm{O}[\mathrm{C} @ @](\mathrm{C})([\mathrm{H}]) \mathrm{CCC}(\mathrm{C})=\mathrm{O}) \mathrm{N}[\mathrm{C} @ @]([\mathrm{H}])(\mathrm{C} / \mathrm{C} 1=\mathrm{C} / \mathrm{C}=\mathrm{CC}=\mathrm{C} 1 \\
)[\mathrm{C} @](\mathrm{O})([\mathrm{H}]) \mathrm{CN}(\mathrm{C}[\mathrm{C} @ @](\mathrm{C})([\mathrm{H}]) \mathrm{CC})[\mathrm{S}](=\mathrm{O})(=\mathrm{O}) \mathrm{C} 3=\mathrm{CC}=\mathrm{C} 2 \mathrm{~N}=\mathrm{C}[ \\
\mathrm{S}] \mathrm{C} 2=\mathrm{C} 3\end{array}$ \\
\hline
\end{tabular}




\begin{tabular}{|c|c|c|c|c|c|c|c|}
\hline H17 & * & 2.856 & 4.729 & 97.484 & 4.103 & 62.847 & $\begin{array}{l}\mathrm{O}=\mathrm{C}(\mathrm{OC}[\mathrm{C} @](\mathrm{C})([\mathrm{H}]) \mathrm{C}(=\mathrm{O}) \mathrm{OC}) \mathrm{N}[\mathrm{C} @ @]([\mathrm{H}])(\mathrm{C} / \mathrm{C} 1=\mathrm{C} / \mathrm{C}=\mathrm{CC}=\mathrm{C} 1)[ \\
\mathrm{C} @](\mathrm{O})([\mathrm{H}]) \mathrm{CN}(\mathrm{C}[\mathrm{C} @ @](\mathrm{C})([\mathrm{H}]) \mathrm{CC})[\mathrm{S}](=\mathrm{O})(=\mathrm{O}) \mathrm{C} 3=\mathrm{CC}=\mathrm{C} 2 \mathrm{~N}=\mathrm{C}[\mathrm{S} \\
] \mathrm{C} 2=\mathrm{C} 3\end{array}$ \\
\hline H31 & $*$ & 1.943 & 2.362 & 48.736 & 2.492 & 38.133 & $\begin{array}{l}\mathrm{O}=\mathrm{C}(\mathrm{O}[\mathrm{C} @ @](\mathrm{C})([\mathrm{H}]) \mathrm{CC}(=\mathrm{O}) \mathrm{OC}) \mathrm{N}[\mathrm{C} @ @]([\mathrm{H}])(\mathrm{C} / \mathrm{C} 1=\mathrm{C} / \mathrm{C}=\mathrm{CC}=\mathrm{C} \\
1)[\mathrm{C} @](\mathrm{O})([\mathrm{H}]) \mathrm{CN}(\mathrm{CCCCC})[\mathrm{S}](=\mathrm{O})(=\mathrm{O}) \mathrm{C} 3=\mathrm{CC}=\mathrm{C} 2 \mathrm{~N}=\mathrm{C}[\mathrm{S}] \mathrm{C} 2=\mathrm{C} 3\end{array}$ \\
\hline H37 & $*$ & 3.664 & 3.602 & 74.278 & 3.686 & 56.438 & $\begin{array}{l}\mathrm{O}=\mathrm{C}(\mathrm{OCCN} 1 \mathrm{CCNC} 1=\mathrm{O}) \mathrm{N}[\mathrm{C} @ @]([\mathrm{H}])(\mathrm{C} / \mathrm{C} 2=\mathrm{C} / \mathrm{C}=\mathrm{CC}=\mathrm{C} 2)[\mathrm{C} @](\mathrm{O}) \\
([\mathrm{H}]) \mathrm{CN}(\mathrm{CCCCC})[\mathrm{S}](=\mathrm{O})(=\mathrm{O}) \mathrm{C} 4=\mathrm{CC}=\mathrm{C} 3 \mathrm{~N}=\mathrm{C}[\mathrm{S}] \mathrm{C} 3=\mathrm{C} 4\end{array}$ \\
\hline H39 & $*$ & 1.376 & 0.531 & 11.015 & 0.635 & 9.656 & $\begin{array}{l}\mathrm{O}=\mathrm{C}(\mathrm{O}[\mathrm{C} @] 1([\mathrm{H}]) \mathrm{CCOC} 1=\mathrm{O}) \mathrm{N}[\mathrm{C} @ @]([\mathrm{H}])(\mathrm{C} / \mathrm{C} 2=\mathrm{C} / \mathrm{C}=\mathrm{CC}=\mathrm{C} 2)[\mathrm{C} \\
@](\mathrm{O})([\mathrm{H}]) \mathrm{CN}(\mathrm{CCCCC})[\mathrm{S}](=\mathrm{O})(=\mathrm{O}) \mathrm{C} 4=\mathrm{CC}=\mathrm{C} 3 \mathrm{~N}=\mathrm{C}[\mathrm{S}] \mathrm{C} 3=\mathrm{C} 4\end{array}$ \\
\hline H49 & $*$ & 3.770 & 3.684 & 75.969 & 3.664 & 56.115 & $\begin{array}{l}\mathrm{O}=\mathrm{C} 10[\mathrm{C} @ @]([\mathrm{H}])(\mathrm{CN} 1 \mathrm{C}=2 \mathrm{C}=\mathrm{C} / \mathrm{C}=\mathrm{C}(/[\mathrm{F}]) \mathrm{C}=2) \mathrm{C}(=\mathrm{O}) \mathrm{N}[\mathrm{C} @ @]([ \\
\mathrm{H}])(\mathrm{C} / \mathrm{C} 3=\mathrm{C} / \mathrm{C}=\mathrm{CC}=\mathrm{C} 3)[\mathrm{C} @ @](\mathrm{O})([\mathrm{H}]) \mathrm{CN}(\mathrm{CC}(\mathrm{C})(\mathrm{C})[\mathrm{H}])[\mathrm{S}](=\mathrm{O})(=\mathrm{O} \\
) \mathrm{C} 4=\mathrm{C} / \mathrm{C}=\mathrm{C}(/ \mathrm{N}) \mathrm{C}=\mathrm{C} 4\end{array}$ \\
\hline H56 & $*$ & 4.796 & 3.783 & 78.004 & 3.594 & 55.028 & $\begin{array}{l}\mathrm{O}=\mathrm{C} 1 \mathrm{O}[\mathrm{C} @ @]([\mathrm{H}])(\mathrm{CN} 1 \mathrm{C}=2 \mathrm{C}=\mathrm{CC}=\mathrm{C}(\mathrm{C}=2) \mathrm{C}([\mathrm{F}])([\mathrm{F}])[\mathrm{F}]) \mathrm{C}(=\mathrm{O}) \mathrm{N}[\mathrm{C} \\
@ @]([\mathrm{H}])(\mathrm{C} / \mathrm{C} 3=\mathrm{C} / \mathrm{C}=\mathrm{CC}=\mathrm{C} 3)[\mathrm{C} @ @](\mathrm{O})([\mathrm{H}]) \mathrm{CN}(\mathrm{CC}(\mathrm{C})(\mathrm{C})[\mathrm{H}])[\mathrm{S}]( \\
=\mathrm{O})(=\mathrm{O}) \mathrm{C} 5=\mathrm{CC}=\mathrm{C} 4 \mathrm{OCOC} 4=\mathrm{C} 5\end{array}$ \\
\hline H62 & $*$ & 3.876 & 4.593 & 94.676 & 4.686 & 71.781 & $\begin{array}{l}\mathrm{O}=\mathrm{C} 1 \mathrm{O}[\mathrm{C} @ @]([\mathrm{H}])(\mathrm{CN} 1 \mathrm{C}=2 \mathrm{C}=\mathrm{CC}=\mathrm{C}(\mathrm{C}=2) \mathrm{C}(=\mathrm{O}) \mathrm{C}) \mathrm{C}(=\mathrm{O}) \mathrm{N}[\mathrm{C} @ @] \\
([\mathrm{H}])(\mathrm{C} / \mathrm{C} 3=\mathrm{C} / \mathrm{C}=\mathrm{CC}=\mathrm{C} 3)[\mathrm{C} @ @](\mathrm{O})([\mathrm{H}]) \mathrm{CN}(\mathrm{CC}(\mathrm{C})(\mathrm{C})[\mathrm{H}])[\mathrm{S}](=\mathrm{O})(= \\
\mathrm{O}) \mathrm{C} 4=\mathrm{C} / \mathrm{C}=\mathrm{C}(\mathrm{OCC}) \mathrm{C}(\mathrm{C})=\mathrm{C} 4\end{array}$ \\
\hline H63 & $*$ & 4.097 & 4.022 & 82.915 & 3.936 & 60.282 & $\begin{array}{l}\mathrm{O}=\mathrm{C} 1 \mathrm{O}[\mathrm{C} @ @]([\mathrm{H}])(\mathrm{CN} 1 \mathrm{C}=2 \mathrm{C}=\mathrm{CC}(=\mathrm{CC}=2) \mathrm{C}(=\mathrm{O}) \mathrm{C}) \mathrm{C}(=\mathrm{O}) \mathrm{N}[\mathrm{C} @ @] \\
([\mathrm{H}])(\mathrm{C} / \mathrm{C} 3=\mathrm{C} / \mathrm{C}=\mathrm{CC}=\mathrm{C} 3)[\mathrm{C} @ @](\mathrm{O})([\mathrm{H}]) \mathrm{CN}(\mathrm{CC}(\mathrm{C})(\mathrm{C})[\mathrm{H}])[\mathrm{S}](=\mathrm{O})(= \\
\mathrm{O}) \mathrm{C} 4=\mathrm{C} / \mathrm{C}=\mathrm{C}(/ \mathrm{OC}) \mathrm{C}(\mathrm{C})=\mathrm{C} 4\end{array}$ \\
\hline H72 & $*$ & 0.724 & 0.846 & 17.507 & 0.781 & 11.889 & $\begin{array}{l}\mathrm{O}=\mathrm{C} 1 \mathrm{O}[\mathrm{C} @ @]([\mathrm{H}])(\mathrm{CN} 1 \mathrm{C}=2 \mathrm{C}=\mathrm{C} / \mathrm{C}=\mathrm{C}(/[\mathrm{F}]) \mathrm{C}=2) \mathrm{C}(=\mathrm{O}) \mathrm{N}[\mathrm{C} @ @]([ \\
\mathrm{H}])(\mathrm{C} / \mathrm{C} 3=\mathrm{C} / \mathrm{C}=\mathrm{CC}=\mathrm{C} 3)[\mathrm{C} @ @](\mathrm{O})([\mathrm{H}]) \mathrm{CN}(\mathrm{C} / \mathrm{C} 4=\mathrm{C} / \mathrm{C}=\mathrm{C}[\mathrm{S}] 4)[\mathrm{S}](= \\
\mathrm{O})(=\mathrm{O}) \mathrm{C} 5=\mathrm{CC}=\mathrm{CC}(=\mathrm{C} 5) \mathrm{OC}\end{array}$ \\
\hline H73 & $*$ & 1.530 & 0.930 & 19.239 & 0.761 & 11.586 & $\begin{array}{l}\mathrm{O}=\mathrm{C} 1 \mathrm{O}[\mathrm{C} @ @]([\mathrm{H}])(\mathrm{CN} 1 \mathrm{C}=2 \mathrm{C}=\mathrm{CC}(=\mathrm{CC}=2) \mathrm{C}(=\mathrm{O}) \mathrm{C}) \mathrm{C}(=\mathrm{O}) \mathrm{N}[\mathrm{C} @ @] \\
([\mathrm{H}])(\mathrm{C} / \mathrm{C} 3=\mathrm{C} / \mathrm{C}=\mathrm{CC}=\mathrm{C} 3)[\mathrm{C} @ @](\mathrm{O})([\mathrm{H}]) \mathrm{CN}(\mathrm{C} / \mathrm{C} 4=\mathrm{C} / \mathrm{C}=\mathrm{C}[\mathrm{S}] 4)[\mathrm{S}]( \\
=0)(=\mathrm{O}) \mathrm{C} 5=\mathrm{CC}=\mathrm{CC}(=\mathrm{C} 5) \mathrm{OC}\end{array}$ \\
\hline H84 & * & 2.833 & 2.182 & 45.015 & 2.144 & 32.792 & $\begin{array}{l}\mathrm{O}=\mathrm{C} 1 \mathrm{O}[\mathrm{C} @ @]([\mathrm{H}])(\mathrm{CN} 1 \mathrm{C}=2 \mathrm{C}=\mathrm{CC}=\mathrm{CC}=2 \mathrm{O}) \mathrm{C}(=\mathrm{O}) \mathrm{N}[\mathrm{C} @ @]([\mathrm{H}])(\mathrm{C} \\
/ \mathrm{C} 3=\mathrm{C} / \mathrm{C}=\mathrm{CC}=\mathrm{C} 3)[\mathrm{C} @ @](\mathrm{O})([\mathrm{H}]) \mathrm{CN}(\mathrm{CC}(\mathrm{C})(\mathrm{C})[\mathrm{H}])[\mathrm{S}](=\mathrm{O})(=\mathrm{O}) \mathrm{C5}= \\
\mathrm{CC}=\mathrm{C} 40 \mathrm{OCOC} 4=\mathrm{C} 5\end{array}$ \\
\hline H92 & $*$ & 3.460 & 3.698 & 76.246 & 3.643 & 55.784 & $\begin{array}{l}\mathrm{O}=\mathrm{C} 1 \mathrm{O}[\mathrm{C} @ @]([\mathrm{H}])(\mathrm{CN} 1 \mathrm{C}=2 \mathrm{C}=\mathrm{CC}([\mathrm{F}])=\mathrm{CC}=2[\mathrm{~F}]) \mathrm{C}(=\mathrm{O}) \mathrm{N}[\mathrm{C} @ @]([ \\
\mathrm{H}])(\mathrm{C} / \mathrm{C} 3=\mathrm{C} / \mathrm{C}=\mathrm{CC}=\mathrm{C} 3)[\mathrm{C} @ @](\mathrm{O})([\mathrm{H}]) \mathrm{CN}(\mathrm{CC}(\mathrm{C})(\mathrm{C})[\mathrm{H}])[\mathrm{S}](=\mathrm{O})(=\mathrm{O} \\
) \mathrm{C} 5=\mathrm{CC}=\mathrm{C} 4 \mathrm{OCOC} 4=\mathrm{C} 5\end{array}$ \\
\hline H103 & $*$ & 3.496 & 3.551 & 73.212 & 3.564 & 54.575 & $\begin{array}{l}\mathrm{O}=\mathrm{C} 1 \mathrm{O}[\mathrm{C} @ @]([\mathrm{H}])(\mathrm{CN} 1 \mathrm{C}=2 \mathrm{C}=\mathrm{C} / \mathrm{C}=\mathrm{C}(/[\mathrm{F}]) \mathrm{C}=2) \mathrm{C}(=\mathrm{O}) \mathrm{N}[\mathrm{C} @ @]([ \\
\mathrm{H}])(\mathrm{C} / \mathrm{C} 3=\mathrm{C} / \mathrm{C}=\mathrm{CC}=\mathrm{C} 3)[\mathrm{C} @ @](\mathrm{O})([\mathrm{H}]) \mathrm{CN}(\mathrm{CC}(\mathrm{C})(\mathrm{C})[\mathrm{H}])[\mathrm{S}](=\mathrm{O})(=\mathrm{O} \\
) \mathrm{C} 4=\mathrm{CC}=\mathrm{C}(\mathrm{C}=\mathrm{C} 4) \mathrm{CO}\end{array}$ \\
\hline H110 & * & 3.622 & 3.686 & 76.000 & 3.756 & 57.524 & $\begin{array}{l}\mathrm{O}=\mathrm{C} 1 \mathrm{O}[\mathrm{C} @ @]([\mathrm{H}])(\mathrm{CN} 1 \mathrm{C}=2 \mathrm{C}=\mathrm{CC}=\mathrm{C}(\mathrm{C}=2) \mathrm{OC}([\mathrm{F}])([\mathrm{F}])[\mathrm{F}]) \mathrm{C}(=\mathrm{O}) \mathrm{N} \\
{[\mathrm{C} @ @]([\mathrm{H}])(\mathrm{C} / \mathrm{C} 3=\mathrm{C} / \mathrm{C}=\mathrm{CC}=\mathrm{C} 3)[\mathrm{C} @ @](\mathrm{O})([\mathrm{H}]) \mathrm{CN}(\mathrm{CC}(\mathrm{C})(\mathrm{C})[\mathrm{H}])[\mathrm{S}} \\
](=\mathrm{S})(=\mathrm{O}) \mathrm{C} 5=\mathrm{CC}=\mathrm{C} 4 \mathrm{OCOC} 4=\mathrm{C} 5\end{array}$ \\
\hline H125 & $*$ & 4.292 & 4.134 & 85.230 & 4.180 & 64.022 & $\begin{array}{l}\mathrm{O}=\mathrm{C} 1 \mathrm{O}[\mathrm{C} @ @]([\mathrm{H}])(\mathrm{CN} 1 \mathrm{C}=2 \mathrm{C}=\mathrm{CC}=\mathrm{C}(\mathrm{C}=2) \mathrm{NC}(=\mathrm{O}) \mathrm{C}) \mathrm{C}(=\mathrm{O}) \mathrm{N}[\mathrm{C} @ \\
@]([\mathrm{H}])(\mathrm{C} / \mathrm{C} 3=\mathrm{C} / \mathrm{C}=\mathrm{CC}=\mathrm{C} 3)[\mathrm{C} @ @](\mathrm{O})([\mathrm{H}]) \mathrm{CN}(\mathrm{CC}(\mathrm{C})(\mathrm{C})[\mathrm{H}])[\mathrm{S}](=\mathrm{C} \\
)(=\mathrm{O}) \mathrm{C} 5=\mathrm{CC}=\mathrm{C} 40 \mathrm{OCOC} 4=\mathrm{C} 5\end{array}$ \\
\hline H128 & $*$ & 3.539 & 3.782 & 77.979 & 3.691 & 56.518 & $\begin{array}{l}\mathrm{O}=\mathrm{C} 1 \mathrm{O}[\mathrm{C} @ @]([\mathrm{H}])(\mathrm{CN} 1 \mathrm{C}=2 \mathrm{C}=\mathrm{CC}=\mathrm{C}(\mathrm{C}=2) \mathrm{NC}(=\mathrm{O}) \mathrm{OC}) \mathrm{C}(=\mathrm{O}) \mathrm{N}[\mathrm{C} @ \\
@]([\mathrm{H}])(\mathrm{C} / \mathrm{C} 3=\mathrm{C} / \mathrm{C}=\mathrm{CC}=\mathrm{C} 3)[\mathrm{C} @ @](\mathrm{O})([\mathrm{H}]) \mathrm{CN}(\mathrm{CC}(\mathrm{C})(\mathrm{C})[\mathrm{H}])[\mathrm{S}](=\mathrm{O} \\
)(=\mathrm{O}) \mathrm{C} 5=\mathrm{CC}=\mathrm{C} 4 \mathrm{OCOC} 4=\mathrm{C} 5\end{array}$ \\
\hline
\end{tabular}

${ }^{1}$ Experimental $p K_{I} ;{ }^{2}$ Calculated $p K_{i}$ 
Table S3: Observed, predicted activity and DCW, and distribution into the training (+), calibration (-), test (\#) and validation (*) sets of split 3 (Models 5 and 6)

\begin{tabular}{|c|c|c|c|c|c|c|c|}
\hline \multirow{2}{*}{$\begin{array}{l}\text { Mol. } \\
\text { No. }\end{array}$} & \multirow{2}{*}{ Set } & \multirow[b]{2}{*}{${ }^{1} \operatorname{Exp}$} & \multicolumn{2}{|c|}{ Model 5} & \multicolumn{2}{|c|}{ Model 6} & \multirow{2}{*}{ SMILES } \\
\hline & & & ${ }^{2}$ Calc. & DCW & ${ }^{2}$ Calc. & DCW & \\
\hline $\mathrm{H} 2$ & + & 3.059 & 2.732 & 59.023 & 2.692 & 68.549 & $\begin{array}{l}\mathrm{O}=\mathrm{C}(\mathrm{O}[\mathrm{C} @ @](\mathrm{C})([\mathrm{H}]) \mathrm{CC}(=\mathrm{O}) \mathrm{OC}) \mathrm{N}[\mathrm{C} @ @]([\mathrm{H}])(\mathrm{C} / \mathrm{C} 1=\mathrm{C} / \\
\mathrm{C}=\mathrm{CC}=\mathrm{C} 1)[\mathrm{C} @](\mathrm{O})([\mathrm{H}]) \mathrm{CN}(\mathrm{C}[\mathrm{C} @ @](\mathrm{C})([\mathrm{H}]) \mathrm{CC})[\mathrm{S}](=\mathrm{O})( \\
=\mathrm{O}) \mathrm{C} 2=\mathrm{CC}=\mathrm{C}(\mathrm{C}=\mathrm{C} 2) \mathrm{OC}\end{array}$ \\
\hline $\mathrm{H} 4$ & + & 5.523 & 5.532 & 119.535 & 5.492 & 139.826 & $\begin{array}{l}\mathrm{O}=\mathrm{C}(\mathrm{OCCNC}(=\mathrm{O}) \mathrm{C}([\mathrm{Cl}])([\mathrm{Cl}])[\mathrm{H}]) \mathrm{N}[\mathrm{C} @ @]([\mathrm{H}])(\mathrm{C} / \mathrm{C} 1=\mathrm{C} / \\
\mathrm{C}=\mathrm{CC}=\mathrm{C} 1)[\mathrm{C} @](\mathrm{O})([\mathrm{H}]) \mathrm{CN}(\mathrm{C}[\mathrm{C} @ @](\mathrm{C})([\mathrm{H}]) \mathrm{CC})[\mathrm{S}](=\mathrm{O})( \\
=\mathrm{O}) \mathrm{C} 2=\mathrm{CC}=\mathrm{C}(\mathrm{C}=\mathrm{C} 2) \mathrm{OC}\end{array}$ \\
\hline H6 & + & 2.697 & 3.311 & 71.535 & 3.310 & 84.267 & $\begin{array}{l}\mathrm{O}=\mathrm{C}(\mathrm{OCCNC}(=\mathrm{O}) \mathrm{OC}(\mathrm{C})(\mathrm{C}) \mathrm{C}) \mathrm{N}[\mathrm{C} @ @]([\mathrm{H}])(\mathrm{C} / \mathrm{C} 1=\mathrm{C} / \mathrm{C}=\mathrm{C} \\
\mathrm{C}=\mathrm{C} 1)[\mathrm{C} @(\mathrm{O})([\mathrm{H}]) \mathrm{CN}(\mathrm{C}[\mathrm{C} @ @](\mathrm{C})([\mathrm{H}]) \mathrm{CC})[\mathrm{S}](=\mathrm{O})(=\mathrm{O}) \\
\mathrm{C} 2=\mathrm{CC}=\mathrm{C}(\mathrm{C}=\mathrm{C} 2) \mathrm{OC}\end{array}$ \\
\hline H8 & + & 4.319 & 4.284 & 92.563 & 4.273 & 108.781 & $\begin{array}{l}\mathrm{O}=\mathrm{C}(\mathrm{OCCN} 1 \mathrm{CCNC} 1=\mathrm{O}) \mathrm{N}[\mathrm{C} @ @]([\mathrm{H}])(\mathrm{C} / \mathrm{C} 2=\mathrm{C} / \mathrm{C}=\mathrm{CC}=\mathrm{C} 2 \\
)[\mathrm{C} @](\mathrm{O})([\mathrm{H}]) \mathrm{CN}(\mathrm{C}[\mathrm{C} @ @](\mathrm{C})([\mathrm{H}]) \mathrm{CC})[\mathrm{S}](=\mathrm{O})(=\mathrm{O}) \mathrm{C} 3=\mathrm{C} \\
\mathrm{C}=\mathrm{C}(\mathrm{C}=\mathrm{C} 3) \mathrm{OC}\end{array}$ \\
\hline H11 & + & 4.260 & 4.262 & 92.091 & 4.264 & 108.555 & $\begin{array}{l}\mathrm{O}=\mathrm{C}(\mathrm{OC}[\mathrm{C} @ @] 1([\mathrm{H}]) \mathrm{NOCC} 1) \mathrm{N}[\mathrm{C} @ @]([\mathrm{H}])(\mathrm{C} / \mathrm{C} 2=\mathrm{C} / \mathrm{C}= \\
\mathrm{CC}=\mathrm{C} 2)[\mathrm{C} @](\mathrm{O})([\mathrm{H}]) \mathrm{CN}(\mathrm{C}[\mathrm{C} @ @](\mathrm{C})([\mathrm{H}]) \mathrm{CC})[\mathrm{S}](=\mathrm{O})(=\mathrm{O} \\
) \mathrm{C} 3=\mathrm{CC}=\mathrm{C}(\mathrm{C}=\mathrm{C} 3) \mathrm{OC}\end{array}$ \\
\hline H12 & + & 3.229 & 2.663 & 57.549 & 2.654 & 67.578 & $\begin{array}{l}\mathrm{O}=\mathrm{C}(\mathrm{O}[\mathrm{C} @ @](\mathrm{C})([\mathrm{H}]) \mathrm{CC}(=\mathrm{O}) \mathrm{OC}) \mathrm{N}[\mathrm{C} @ @]([\mathrm{H}])(\mathrm{C} / \mathrm{C} 1=\mathrm{C} / \\
\mathrm{C}=\mathrm{CC}=\mathrm{C} 1)[\mathrm{C} @](\mathrm{O})([\mathrm{H}]) \mathrm{CN}(\mathrm{C}[\mathrm{C} @ @](\mathrm{C})([\mathrm{H}]) \mathrm{CC})[\mathrm{S}](=\mathrm{O})( \\
=\mathrm{O}) \mathrm{C} 3=\mathrm{CC}=\mathrm{C} 2 \mathrm{~N}=\mathrm{C}[\mathrm{S}] \mathrm{C} 2=\mathrm{C} 3\end{array}$ \\
\hline H14 & + & 4.222 & 5.464 & 118.062 & 5.454 & 138.855 & $\begin{array}{l}\mathrm{O}=\mathrm{C}(\mathrm{OCCNC}(=\mathrm{O}) \mathrm{C}([\mathrm{Cl}])([\mathrm{Cl}])[\mathrm{H}]) \mathrm{N}[\mathrm{C} @ @]([\mathrm{H}])(\mathrm{C} / \mathrm{C} 1=\mathrm{C} / \\
\mathrm{C}=\mathrm{CC}=\mathrm{C} 1)[\mathrm{C} @](\mathrm{O})([\mathrm{H}]) \mathrm{CN}(\mathrm{C}[\mathrm{C} @ @](\mathrm{C})([\mathrm{H}]) \mathrm{CC})[\mathrm{S}](=\mathrm{O})( \\
=\mathrm{O}) \mathrm{C} 3=\mathrm{CC}=\mathrm{C} 2 \mathrm{~N}=\mathrm{C}[\mathrm{S}] \mathrm{C} 2=\mathrm{C} 3\end{array}$ \\
\hline H16 & + & 3.638 & 3.243 & 70.061 & 3.272 & 83.296 & $\begin{array}{l}\mathrm{O}=\mathrm{C}(\mathrm{OCCNC}(=\mathrm{O}) \mathrm{OC}(\mathrm{C})(\mathrm{C}) \mathrm{C}) \mathrm{N}[\mathrm{C} @ @]([\mathrm{H}])(\mathrm{C} / \mathrm{C} 1=\mathrm{C} / \mathrm{C}=\mathrm{C} \\
\mathrm{C}=\mathrm{C} 1)[\mathrm{C} @(\mathrm{O})([\mathrm{H}]) \mathrm{CN}(\mathrm{C}[\mathrm{C} @(\mathrm{C})([\mathrm{H}]) \mathrm{CC})[\mathrm{S}](=\mathrm{O})(=\mathrm{O}) \\
\mathrm{C} 3=\mathrm{CC}=\mathrm{C} 2 \mathrm{~N}=\mathrm{C}[\mathrm{S}] \mathrm{C} 2=\mathrm{C} 3\end{array}$ \\
\hline H18 & + & 5.301 & 4.712 & 101.812 & 4.735 & 120.566 & $\begin{array}{l}\mathrm{O}=\mathrm{C}(\mathrm{OCCN} 1 \mathrm{CCNC} 1=\mathrm{O}) \mathrm{N}[\mathrm{C} @ @]([\mathrm{H}])(\mathrm{C} / \mathrm{C} 2=\mathrm{C} / \mathrm{C}=\mathrm{CC}=\mathrm{C} 2 \\
)[\mathrm{C} @](\mathrm{O})([\mathrm{H}]) \mathrm{CN}(\mathrm{C}[\mathrm{C} @ @](\mathrm{C})([\mathrm{H}]) \mathrm{CC})[\mathrm{S}](=\mathrm{O})(=\mathrm{O}) \mathrm{C} 4=\mathrm{C} \\
\mathrm{C}=\mathrm{C} 3 \mathrm{~N}=\mathrm{C}[\mathrm{S}] \mathrm{C} 3=\mathrm{C} 4\end{array}$ \\
\hline H19 & + & 5.301 & 5.823 & 125.807 & 5.875 & 149.576 & $\begin{array}{l}\mathrm{O}=\mathrm{C}(\mathrm{OCCN} 1 \mathrm{CCOC} 1=\mathrm{O}) \mathrm{N}[\mathrm{C} @ @]([\mathrm{H}])(\mathrm{C} / \mathrm{C} 2=\mathrm{C} / \mathrm{C}=\mathrm{CC}=\mathrm{C} 2 \\
)[\mathrm{C} @](\mathrm{O})([\mathrm{H}]) \mathrm{CN}(\mathrm{C}[\mathrm{C} @ @](\mathrm{C})([\mathrm{H}]) \mathrm{CC})[\mathrm{S}](=\mathrm{O})(=\mathrm{O}) \mathrm{C} 4=\mathrm{C} \\
\mathrm{C}=\mathrm{C} 3 \mathrm{~N}=\mathrm{C}[\mathrm{S}] \mathrm{C} 3=\mathrm{C} 4\end{array}$ \\
\hline $\mathrm{H} 21$ & + & 5.523 & 5.014 & 108.326 & 5.040 & 128.318 & $\begin{array}{l}\mathrm{O}=\mathrm{C}(\mathrm{OC}[\mathrm{C} @] 1([\mathrm{H}]) \mathrm{NC}(=\mathrm{O}) \mathrm{CC} 1) \mathrm{N}[\mathrm{C} @ @]([\mathrm{H}])(\mathrm{C} / \mathrm{C} 2=\mathrm{C} / \mathrm{C} \\
=\mathrm{CC}=\mathrm{C} 2)[\mathrm{C} @](\mathrm{O})([\mathrm{H}]) \mathrm{CN}(\mathrm{C}[\mathrm{C} @ @](\mathrm{C})([\mathrm{H}]) \mathrm{CC})[\mathrm{S}](=\mathrm{O})(= \\
\mathrm{O}) \mathrm{C} 4=\mathrm{CC}=\mathrm{C} 3 \mathrm{~N}=\mathrm{C}[\mathrm{S}] \mathrm{C} 3=\mathrm{C} 4\end{array}$ \\
\hline $\mathrm{H} 22$ & + & 1.763 & 2.073 & 44.799 & 2.014 & 51.272 & $\begin{array}{l}\mathrm{O}=\mathrm{C}(\mathrm{O}[\mathrm{C} @ @](\mathrm{C})([\mathrm{H}]) \mathrm{CC}(=\mathrm{O}) \mathrm{OC}) \mathrm{N}[\mathrm{C} @ @]([\mathrm{H}])(\mathrm{C} / \mathrm{C} 1=\mathrm{C} / \\
\mathrm{C}=\mathrm{CC}=\mathrm{C} 1)[\mathrm{C} @](\mathrm{O})([\mathrm{H}]) \mathrm{CN}(\mathrm{CCCCC})[\mathrm{S}](=\mathrm{O})(=\mathrm{O}) \mathrm{C} 2=\mathrm{CC}= \\
\mathrm{C}(\mathrm{C}=\mathrm{C} 2) \mathrm{OC}\end{array}$ \\
\hline $\mathrm{H} 24$ & + & 2.167 & 2.293 & 49.545 & 2.475 & 63.017 & $\begin{array}{l}\mathrm{O}=\mathrm{C}(\mathrm{O}[\mathrm{C} @ @](\mathrm{C})([\mathrm{H}]) \mathrm{CCC}(\mathrm{C})=\mathrm{O}) \mathrm{N}[\mathrm{C} @ @]([\mathrm{H}])(\mathrm{C} / \mathrm{C} 1=\mathrm{C} / \\
\mathrm{C}=\mathrm{CC}=\mathrm{C} 1)[\mathrm{C} @](\mathrm{O})([\mathrm{H}]) \mathrm{CN}(\mathrm{CCCCC})[\mathrm{S}](=\mathrm{O})(=\mathrm{O}) \mathrm{C} 2=\mathrm{CC}= \\
\mathrm{C}(\mathrm{C}=\mathrm{C} 2) \mathrm{OC}\end{array}$ \\
\hline $\mathrm{H} 26$ & + & 3.391 & 3.336 & 72.071 & 3.330 & 84.780 & $\begin{array}{l}\mathrm{O}=\mathrm{C}(\mathrm{OC}[\mathrm{C} @](\mathrm{C})([\mathrm{H}]) \mathrm{C}(=\mathrm{O}) \mathrm{OC}) \mathrm{N}[\mathrm{C} @ @]([\mathrm{H}])(\mathrm{C} / \mathrm{C} 1=\mathrm{C} / \mathrm{C} \\
=\mathrm{CC}=\mathrm{C} 1)[\mathrm{C} @](\mathrm{O})([\mathrm{H}]) \mathrm{CN}(\mathrm{CCCCC})[\mathrm{S}](=\mathrm{O})(=\mathrm{O}) \mathrm{C} 2=\mathrm{CC}=\mathrm{C} \\
(\mathrm{C}=\mathrm{C} 2) \mathrm{OC}\end{array}$ \\
\hline $\mathrm{H} 28$ & + & 5.097 & 4.736 & 102.335 & 4.733 & 120.515 & $\begin{array}{l}\mathrm{O}=\mathrm{C}(\mathrm{OCCN} 1 \mathrm{CCOC} 1=\mathrm{O}) \mathrm{N}[\mathrm{C} @ @]([\mathrm{H}])(\mathrm{C} / \mathrm{C} 2=\mathrm{C} / \mathrm{C}=\mathrm{CC}=\mathrm{C} 2 \\
)[\mathrm{C} @](\mathrm{O})([\mathrm{H}]) \mathrm{CN}(\mathrm{CCCCC})[\mathrm{S}](=\mathrm{O})(=\mathrm{O}) \mathrm{C} 3=\mathrm{CC}=\mathrm{C}(\mathrm{C}=\mathrm{C} 3) \mathrm{O} \\
\mathrm{C}\end{array}$ \\
\hline
\end{tabular}




\begin{tabular}{|c|c|c|c|c|c|c|c|}
\hline $\mathrm{H} 30$ & + & 3.205 & 3.927 & 84.854 & 3.898 & 99.256 & $\begin{array}{l}\mathrm{O}=\mathrm{C}(\mathrm{OC}[\mathrm{C} @] 1([\mathrm{H}]) \mathrm{NC}(=\mathrm{O}) \mathrm{CC} 1) \mathrm{N}[\mathrm{C} @ @]([\mathrm{H}])(\mathrm{C} / \mathrm{C} 2=\mathrm{C} / \mathrm{C} \\
=\mathrm{CC}=\mathrm{C} 2)[\mathrm{C} @](\mathrm{O})([\mathrm{H}]) \mathrm{CN}(\mathrm{CCCC})[\mathrm{S}](=\mathrm{O})(=\mathrm{O}) \mathrm{C} 3=\mathrm{CC}=\mathrm{C} \\
(\mathrm{C}=\mathrm{C} 3) \mathrm{OC}\end{array}$ \\
\hline H31 & + & 1.943 & 2.005 & 43.325 & 1.976 & 50.302 & $\begin{array}{l}\mathrm{O}=\mathrm{C}(\mathrm{O}[\mathrm{C} @ @](\mathrm{C})([\mathrm{H}]) \mathrm{CC}(=\mathrm{O}) \mathrm{OC}) \mathrm{N}[\mathrm{C} @ @]([\mathrm{H}])(\mathrm{C} / \mathrm{C} 1=\mathrm{C} / \\
\mathrm{C}=\mathrm{CC}=\mathrm{C} 1)[\mathrm{C} @](\mathrm{O})([\mathrm{H}]) \mathrm{CN}(\mathrm{CCCCC})[\mathrm{S}](=\mathrm{O})(=\mathrm{O}) \mathrm{C} 3=\mathrm{CC}= \\
\mathrm{C} 2 \mathrm{~N}=\mathrm{C}[\mathrm{S}] \mathrm{C} 2=\mathrm{C} 3\end{array}$ \\
\hline H33 & + & 6.000 & 4.806 & 103.837 & 4.775 & 121.579 & $\begin{array}{l}\mathrm{O}=\mathrm{C}(\mathrm{OCCNC}(=\mathrm{O}) \mathrm{C}([\mathrm{Cl}])([\mathrm{Cl}])[\mathrm{H}]) \mathrm{N}[\mathrm{C} @ @]([\mathrm{H}])(\mathrm{C} / \mathrm{C} 1=\mathrm{C} / \\
\mathrm{C}=\mathrm{CC}=\mathrm{C} 1)[\mathrm{C} @](\mathrm{O})([\mathrm{H}]) \mathrm{CN}(\mathrm{CCCCC})[\mathrm{S}](=\mathrm{O})(=\mathrm{O}) \mathrm{C} 3=\mathrm{CC}= \\
\mathrm{C} 2 \mathrm{~N}=\mathrm{C}[\mathrm{S}] \mathrm{C} 2=\mathrm{C} 3\end{array}$ \\
\hline H35 & + & 2.889 & 2.584 & 55.837 & 2.593 & 66.020 & $\begin{array}{l}\mathrm{O}=\mathrm{C}(\mathrm{OCCNC}(=\mathrm{O}) \mathrm{OC}(\mathrm{C})(\mathrm{C}) \mathrm{C}) \mathrm{N}[\mathrm{C} @ @]([\mathrm{H}])(\mathrm{C} / \mathrm{C} 1=\mathrm{C} / \mathrm{C}=\mathrm{C} \\
\mathrm{C}=\mathrm{C} 1)[\mathrm{C} @](\mathrm{O})([\mathrm{H}]) \mathrm{CN}(\mathrm{CCCCC})[\mathrm{S}](=\mathrm{O})(=\mathrm{O}) \mathrm{C} 3=\mathrm{CC}=\mathrm{C} 2 \mathrm{~N} \\
=\mathrm{C}[\mathrm{S}] \mathrm{C} 2=\mathrm{C} 3\end{array}$ \\
\hline H36 & + & 2.476 & 3.267 & 70.598 & 3.292 & 83.810 & $\begin{array}{l}\mathrm{O}=\mathrm{C}(\mathrm{OC}[\mathrm{C} @](\mathrm{C})([\mathrm{H}]) \mathrm{C}(=\mathrm{O}) \mathrm{OC}) \mathrm{N}[\mathrm{C} @ @]([\mathrm{H}])(\mathrm{C} / \mathrm{C} 1=\mathrm{C} / \mathrm{C} \\
=\mathrm{CC}=\mathrm{C} 1)[\mathrm{C} @](\mathrm{O})([\mathrm{H}]) \mathrm{CN}(\mathrm{CCCC})[\mathrm{S}](=\mathrm{O})(=\mathrm{O}) \mathrm{C} 3=\mathrm{CC}=\mathrm{C} \\
2 \mathrm{~N}=\mathrm{C}[\mathrm{S}] \mathrm{C} 2=\mathrm{C} 3\end{array}$ \\
\hline H37 & + & 3.664 & 4.054 & 87.588 & 4.057 & 103.290 & $\begin{array}{l}\mathrm{O}=\mathrm{C}(\mathrm{OCCN} 1 \mathrm{CCNC} 1=\mathrm{O}) \mathrm{N}[\mathrm{C} @ @]([\mathrm{H}])(\mathrm{C} / \mathrm{C} 2=\mathrm{C} / \mathrm{C}=\mathrm{CC}=\mathrm{C} 2 \\
)[\mathrm{C} @](\mathrm{O})([\mathrm{H}]) \mathrm{CN}(\mathrm{CCCCC})[\mathrm{S}](=\mathrm{O})(=\mathrm{O}) \mathrm{C} 4=\mathrm{CC}=\mathrm{C} 3 \mathrm{~N}=\mathrm{C}[\mathrm{S}] \\
\mathrm{C} 3=\mathrm{C} 4\end{array}$ \\
\hline H38 & + & 4.770 & 5.164 & 111.583 & 5.196 & 132.300 & $\begin{array}{l}\mathrm{O}=\mathrm{C}(\mathrm{OCCN} 1 \mathrm{CCOC} 1=\mathrm{O}) \mathrm{N}[\mathrm{C} @ @]([\mathrm{H}])(\mathrm{C} / \mathrm{C} 2=\mathrm{C} / \mathrm{C}=\mathrm{CC}=\mathrm{C} 2 \\
)[\mathrm{C} @](\mathrm{O})([\mathrm{H}]) \mathrm{CN}(\mathrm{CCCCC})[\mathrm{S}](=\mathrm{O})(=\mathrm{O}) \mathrm{C} 4=\mathrm{CC}=\mathrm{C} 3 \mathrm{~N}=\mathrm{C}[\mathrm{S}] \\
\mathrm{C} 3=\mathrm{C} 4\end{array}$ \\
\hline $\mathrm{H} 40$ & + & 4.569 & 4.355 & 94.102 & 4.361 & 111.041 & $\begin{array}{l}\mathrm{O}=\mathrm{C}(\mathrm{OC}[\mathrm{C} @] 1([\mathrm{H}]) \mathrm{NC}(=\mathrm{O}) \mathrm{CC} 1) \mathrm{N}[\mathrm{C} @ @]([\mathrm{H}])(\mathrm{C} / \mathrm{C} 2=\mathrm{C} / \mathrm{C} \\
=\mathrm{CC}=\mathrm{C} 2)[\mathrm{C} @](\mathrm{O})([\mathrm{H}]) \mathrm{CN}(\mathrm{CCCC})[\mathrm{S}](=\mathrm{O})(=\mathrm{O}) \mathrm{C} 4=\mathrm{CC}=\mathrm{C} \\
3 \mathrm{~N}=\mathrm{C}[\mathrm{S}] \mathrm{C} 3=\mathrm{C} 4\end{array}$ \\
\hline $\mathrm{H} 42$ & + & 4.081 & 4.082 & 88.190 & 4.076 & 103.776 & $\begin{array}{l}\mathrm{O}=\mathrm{C} 1 \mathrm{O}[\mathrm{C} @ @]([\mathrm{H}])(\mathrm{CN} 1 \mathrm{C}=2 \mathrm{C}=\mathrm{C} / \mathrm{C}=\mathrm{C}(/[\mathrm{F}]) \mathrm{C}=2) \mathrm{C}(=\mathrm{O}) \mathrm{N}[ \\
\mathrm{C} @ @]([\mathrm{H}])(\mathrm{C} / \mathrm{C} 3=\mathrm{C} / \mathrm{C}=\mathrm{CC}=\mathrm{C} 3)[\mathrm{C} @ @](\mathrm{O})([\mathrm{H}]) \mathrm{CN}(\mathrm{CC}(\mathrm{C}) \\
(\mathrm{C})[\mathrm{H}])[\mathrm{S}](=\mathrm{O})(=\mathrm{O}) \mathrm{C} 4=\mathrm{CC}=\mathrm{C}(\mathrm{C}=\mathrm{C} 4) \mathrm{OC}\end{array}$ \\
\hline $\mathrm{H} 45$ & + & 6.097 & 4.729 & 102.178 & 4.753 & 121.010 & $\begin{array}{l}\mathrm{O}=\mathrm{C} 1 \mathrm{O}[\mathrm{C} @ @]([\mathrm{H}])(\mathrm{CN} 1 \mathrm{C}=2 \mathrm{C}=\mathrm{CC}=\mathrm{C}(\mathrm{C}=2) \mathrm{C}(\mathrm{C})=\mathrm{O}) \mathrm{C}(=\mathrm{O} \\
) \mathrm{N}[\mathrm{C} @ @]([\mathrm{H}])(\mathrm{C} / \mathrm{C} 3=\mathrm{C} / \mathrm{C}=\mathrm{CC}=\mathrm{C} 3)[\mathrm{C} @ @](\mathrm{O})([\mathrm{H}]) \mathrm{CN}(\mathrm{CC} \\
(\mathrm{C})(\mathrm{C})[\mathrm{H}])[\mathrm{S}](=\mathrm{O})(=\mathrm{O}) \mathrm{C} 4=\mathrm{CC}=\mathrm{C}(\mathrm{C}=\mathrm{C} 4) \mathrm{OC}\end{array}$ \\
\hline H46 & + & 5.398 & 4.348 & 93.946 & 4.380 & 111.522 & $\begin{array}{l}\mathrm{O}=\mathrm{C} 1 \mathrm{O}[\mathrm{C} @ @]([\mathrm{H}])(\mathrm{CN} 1 \mathrm{C}=2 \mathrm{C}=\mathrm{CC}(=\mathrm{CC}=2) \mathrm{C}(\mathrm{C})=\mathrm{O}) \mathrm{C}(=\mathrm{O} \\
) \mathrm{N}[\mathrm{C} @ @]([\mathrm{H}])(\mathrm{C} / \mathrm{C} 3=\mathrm{C} / \mathrm{C}=\mathrm{CC}=\mathrm{C} 3)[\mathrm{C} @ @](\mathrm{O})([\mathrm{H}]) \mathrm{CN}(\mathrm{CC} \\
(\mathrm{C})(\mathrm{C})[\mathrm{H}])[\mathrm{S}](=\mathrm{O})(=\mathrm{O}) \mathrm{C} 4=\mathrm{CC}=\mathrm{C}(\mathrm{C}=\mathrm{C} 4) \mathrm{OC}\end{array}$ \\
\hline H49 & + & 3.770 & 3.642 & 78.681 & 3.594 & 91.513 & $\begin{array}{l}\mathrm{O}=\mathrm{C} 1 \mathrm{O}[\mathrm{C} @ @]([\mathrm{H}])(\mathrm{CN} 1 \mathrm{C}=2 \mathrm{C}=\mathrm{C} / \mathrm{C}=\mathrm{C}(/[\mathrm{F}]) \mathrm{C}=2) \mathrm{C}(=\mathrm{O}) \mathrm{N}[ \\
\mathrm{C} @ @]([\mathrm{H}])(\mathrm{C} / \mathrm{C} 3=\mathrm{C} / \mathrm{C}=\mathrm{CC}=\mathrm{C} 3)[\mathrm{C} @ @](\mathrm{O})([\mathrm{H}]) \mathrm{CN}(\mathrm{CC}(\mathrm{C}) \\
(\mathrm{C})[\mathrm{H}])[\mathrm{S}](=\mathrm{O})(=\mathrm{O}) \mathrm{C} 4=\mathrm{C} / \mathrm{C}=\mathrm{C}(/ \mathrm{N}) \mathrm{C}=\mathrm{C} 4\end{array}$ \\
\hline H51 & + & 4.377 & 3.455 & 74.651 & 3.428 & 87.280 & $\begin{array}{l}\mathrm{O}=\mathrm{C} 1 \mathrm{O}[\mathrm{C} @ @]([\mathrm{H}])(\mathrm{CN} 1 \mathrm{C}=2 \mathrm{C}=\mathrm{CC}=\mathrm{C}(\mathrm{C}=2) \mathrm{C}([\mathrm{F}])([\mathrm{F}])[\mathrm{F}]) \\
\mathrm{C}(=\mathrm{O}) \mathrm{N}[\mathrm{C} @ @]([\mathrm{H}])(\mathrm{C} / \mathrm{C} 3=\mathrm{C} / \mathrm{C}=\mathrm{CC}=\mathrm{C} 3)[\mathrm{C} @ @](\mathrm{O})([\mathrm{H}]) \mathrm{C} \\
\mathrm{N}(\mathrm{CC}(\mathrm{C})(\mathrm{C})[\mathrm{H}])[\mathrm{S}](=\mathrm{O})(=\mathrm{O}) \mathrm{C} 4=\mathrm{C} / \mathrm{C}=\mathrm{C}(/ \mathrm{N}) \mathrm{C}=\mathrm{C} 4\end{array}$ \\
\hline H53 & + & 3.735 & 3.908 & 84.438 & 3.899 & 99.259 & $\begin{array}{l}\mathrm{O}=\mathrm{C} 1 \mathrm{O}[\mathrm{C} @ @]([\mathrm{H}])(\mathrm{CN} 1 \mathrm{C}=2 \mathrm{C}=\mathrm{CC}(=\mathrm{CC}=2) \mathrm{C}(=\mathrm{O}) \mathrm{C}) \mathrm{C}(=\mathrm{O} \\
) \mathrm{N}[\mathrm{C} @ @]([\mathrm{H}])(\mathrm{C} / \mathrm{C} 3=\mathrm{C} / \mathrm{C}=\mathrm{CC}=\mathrm{C} 3)[\mathrm{C} @ @](\mathrm{O})([\mathrm{H}]) \mathrm{CN}(\mathrm{CC} \\
(\mathrm{C})(\mathrm{C})[\mathrm{H}])[\mathrm{S}](=\mathrm{O})(=\mathrm{O}) \mathrm{C} 4=\mathrm{C} / \mathrm{C}=\mathrm{C}(/ \mathrm{N}) \mathrm{C}=\mathrm{C} 4\end{array}$ \\
\hline H56 & + & 4.796 & 4.023 & 86.924 & 4.028 & 102.542 & $\begin{array}{l}\mathrm{O}=\mathrm{C} 1 \mathrm{O}[\mathrm{C} @ @]([\mathrm{H}])(\mathrm{CN} 1 \mathrm{C}=2 \mathrm{C}=\mathrm{CC}=\mathrm{C}(\mathrm{C}=2) \mathrm{C}([\mathrm{F}])([\mathrm{F}])[\mathrm{F}]) \\
\mathrm{C}(=\mathrm{O}) \mathrm{N}[\mathrm{C} @ @]([\mathrm{H}])(\mathrm{C} / \mathrm{C} 3=\mathrm{C} / \mathrm{C}=\mathrm{CC}=\mathrm{C} 3)[\mathrm{C} @ @](\mathrm{O})([\mathrm{H}]) \mathrm{C} \\
\mathrm{N}(\mathrm{CC}(\mathrm{C})(\mathrm{C})[\mathrm{H}])[\mathrm{S}](=\mathrm{O})(=\mathrm{O}) \mathrm{C} 5=\mathrm{CC}=\mathrm{C} 4 \mathrm{OCOC} 4=\mathrm{C} 5\end{array}$ \\
\hline H57 & + & 5.222 & 4.857 & 104.942 & 4.871 & 124.009 & $\begin{array}{l}\mathrm{O}=\mathrm{C} 1 \mathrm{O}[\mathrm{C} @ @]([\mathrm{H}])(\mathrm{CN} 1 \mathrm{C}=2 \mathrm{C}=\mathrm{CC}=\mathrm{C}(\mathrm{C}=2) \mathrm{C}(=\mathrm{O}) \mathrm{C}) \mathrm{C}(=\mathrm{O} \\
) \mathrm{N}[\mathrm{C} @ @]([\mathrm{H}])(\mathrm{C} / \mathrm{C} 3=\mathrm{C} / \mathrm{C}=\mathrm{CC}=\mathrm{C} 3)[\mathrm{C} @ @](\mathrm{O})([\mathrm{H}]) \mathrm{CN}(\mathrm{CC} \\
(\mathrm{C})(\mathrm{C})[\mathrm{H}])[\mathrm{S}](=\mathrm{O})(=\mathrm{O}) \mathrm{C} 5=\mathrm{CC}=\mathrm{C} 4 \mathrm{OCOC} 4=\mathrm{C} 5\end{array}$ \\
\hline H59 & + & 4.155 & 3.629 & 78.419 & 3.604 & 91.756 & $\mathrm{O}=\mathrm{C} 1 \mathrm{O}[\mathrm{C} @ @]([\mathrm{H}])(\mathrm{CN} 1 \mathrm{C}=2 \mathrm{C}=\mathrm{C} / \mathrm{C}=\mathrm{C}(/[\mathrm{F}]) \mathrm{C}=2) \mathrm{C}(=\mathrm{O}) \mathrm{N}[$ \\
\hline
\end{tabular}




\begin{tabular}{|c|c|c|c|c|c|c|c|}
\hline & & & & & & & $\begin{array}{l}\mathrm{C} @ @]([\mathrm{H}])(\mathrm{C} / \mathrm{C} 3=\mathrm{C} / \mathrm{C}=\mathrm{CC}=\mathrm{C} 3)[\mathrm{C} @ @](\mathrm{O})([\mathrm{H}]) \mathrm{CN}(\mathrm{CC}(\mathrm{C}) \\
(\mathrm{C})[\mathrm{H}])[\mathrm{S}](=\mathrm{O})(=\mathrm{O}) \mathrm{C} 4=\mathrm{C} / \mathrm{C}=\mathrm{C}(/ \mathrm{OC}) \mathrm{C}(\mathrm{C})=\mathrm{C} 4\end{array}$ \\
\hline H50 & + & 3.465 & 3.779 & 81.660 & 3.801 & 96.780 & $\begin{array}{l}\mathrm{O}=\mathrm{C} 1 \mathrm{O}[\mathrm{C} @ @]([\mathrm{H}])(\mathrm{CN} 1 \mathrm{C}=2 \mathrm{C}=\mathrm{CC}([\mathrm{F}])=\mathrm{C}([\mathrm{F}]) \mathrm{C}=2) \mathrm{C}(=\mathrm{O}) \\
\mathrm{N}[\mathrm{C} @ @]([\mathrm{H}])(\mathrm{C} / \mathrm{C} 3=\mathrm{C} / \mathrm{C}=\mathrm{CC}=\mathrm{C} 3)[\mathrm{C} @ @](\mathrm{O})([\mathrm{H}]) \mathrm{CN}(\mathrm{CC}( \\
\mathrm{C})(\mathrm{C})[\mathrm{H}])[\mathrm{S}](=\mathrm{O})(=\mathrm{O}) \mathrm{C} 4=\mathrm{C} / \mathrm{C}=\mathrm{C}(/ \mathrm{OC}) \mathrm{C}(\mathrm{C})=\mathrm{C} 4\end{array}$ \\
\hline H62 & + & 3.876 & 4.277 & 92.407 & 4.281 & 108.990 & $\begin{array}{l}\mathrm{O}=\mathrm{C} 1 \mathrm{O}[\mathrm{C} @ @]([\mathrm{H}])(\mathrm{CN} 1 \mathrm{C}=2 \mathrm{C}=\mathrm{CC}=\mathrm{C}(\mathrm{C}=2) \mathrm{C}(=\mathrm{O}) \mathrm{C}) \mathrm{C}(=\mathrm{O} \\
) \mathrm{N}[\mathrm{C} @ @]([\mathrm{H}])(\mathrm{C} / \mathrm{C} 3=\mathrm{C} / \mathrm{C}=\mathrm{CC}=\mathrm{C} 3)[\mathrm{C} @ @](\mathrm{O})([\mathrm{H}]) \mathrm{CN}(\mathrm{CC} \\
(\mathrm{C})(\mathrm{C})[\mathrm{H}])[\mathrm{S}](=\mathrm{O})(=\mathrm{O}) \mathrm{C} 4=\mathrm{C} / \mathrm{C}=\mathrm{C}(/ \mathrm{OC}) \mathrm{C}(\mathrm{C})=\mathrm{C} 4\end{array}$ \\
\hline H63 & + & 4.097 & 3.896 & 84.176 & 3.908 & 99.502 & $\begin{array}{l}\mathrm{O}=\mathrm{C} 1 \mathrm{O}[\mathrm{C} @ @]([\mathrm{H}])(\mathrm{CN} 1 \mathrm{C}=2 \mathrm{C}=\mathrm{CC}(=\mathrm{CC}=2) \mathrm{C}(=\mathrm{O}) \mathrm{C}) \mathrm{C}(=\mathrm{O} \\
) \mathrm{N}[\mathrm{C} @ @]([\mathrm{H}])(\mathrm{C} / \mathrm{C} 3=\mathrm{C} / \mathrm{C}=\mathrm{CC}=\mathrm{C} 3)[\mathrm{C} @ @](\mathrm{O})([\mathrm{H}]) \mathrm{CN}(\mathrm{CC} \\
(\mathrm{C})(\mathrm{C})[\mathrm{H}])[\mathrm{S}](=\mathrm{O})(=\mathrm{O}) \mathrm{C} 4=\mathrm{C} / \mathrm{C}=\mathrm{C}(/ \mathrm{OC}) \mathrm{C}(\mathrm{C})=\mathrm{C} 4\end{array}$ \\
\hline H65 & + & 2.699 & 3.548 & 76.654 & 3.477 & 88.536 & $\begin{array}{l}\mathrm{O}=\mathrm{C} 1 \mathrm{O}[\mathrm{C} @ @]([\mathrm{H}])(\mathrm{CN} 1 \mathrm{C}=2 \mathrm{C}=\mathrm{CC}=\mathrm{C}(\mathrm{C}=2) \mathrm{C}(=\mathrm{O}) \mathrm{C}) \mathrm{C}(=\mathrm{O} \\
) \mathrm{N}[\mathrm{C} @ @]([\mathrm{H}])(\mathrm{C} / \mathrm{C} 3=\mathrm{C} / \mathrm{C}=\mathrm{CC}=\mathrm{C} 3)[\mathrm{C} @ @](\mathrm{O})([\mathrm{H}]) \mathrm{CN}(\mathrm{CC} \\
(\mathrm{C})(\mathrm{C})[\mathrm{H}])[\mathrm{S}](=\mathrm{O})(=\mathrm{O}) \mathrm{C} 4=\mathrm{CC}=\mathrm{C}(\mathrm{C}=\mathrm{C} 4) \mathrm{OC}([\mathrm{F}])([\mathrm{F}])[\mathrm{F}]\end{array}$ \\
\hline H67 & + & 3.076 & 3.167 & 68.422 & 3.105 & 79.048 & $\begin{array}{l}\mathrm{O}=\mathrm{C} 1 \mathrm{O}[\mathrm{C} @ @]([\mathrm{H}])(\mathrm{CN} 1 \mathrm{C}=2 \mathrm{C}=\mathrm{CC}(=\mathrm{CC}=2) \mathrm{C}(=\mathrm{O}) \mathrm{C}) \mathrm{C}(=\mathrm{O} \\
) \mathrm{N}[\mathrm{C} @ @]([\mathrm{H}])(\mathrm{C} / \mathrm{C} 3=\mathrm{C} / \mathrm{C}=\mathrm{CC}=\mathrm{C} 3)[\mathrm{C} @ @](\mathrm{O})([\mathrm{H}]) \mathrm{CN}(\mathrm{CC} \\
(\mathrm{C})(\mathrm{C})[\mathrm{H}])[\mathrm{S}](=\mathrm{O})(=\mathrm{O}) \mathrm{C} 4=\mathrm{CC}=\mathrm{C}(\mathrm{C}=\mathrm{C} 4) \mathrm{OC}([\mathrm{F}])([\mathrm{F}])[\mathrm{F}]\end{array}$ \\
\hline H69 & + & 3.237 & 3.100 & 66.979 & 3.087 & 78.587 & $\begin{array}{l}\mathrm{O}=\mathrm{C} 1 \mathrm{O}[\mathrm{C} @ @]([\mathrm{H}])(\mathrm{CN} 1 \mathrm{C}=2 \mathrm{C}=\mathrm{CC}([\mathrm{F}])=\mathrm{C}([\mathrm{F}]) \mathrm{C}=2) \mathrm{C}(=\mathrm{O}) \\
\mathrm{N}[\mathrm{C} @ @]([\mathrm{H}])(\mathrm{C} / \mathrm{C} 3=\mathrm{C} / \mathrm{C}=\mathrm{CC}=\mathrm{C} 3)[\mathrm{C} @ @](\mathrm{O})([\mathrm{H}]) \mathrm{CN}(\mathrm{C}[\mathrm{C} \\
@ @] 4([\mathrm{H}]) \mathrm{CC} 4)[\mathrm{S}](=\mathrm{O})(=\mathrm{O}) \mathrm{C} 5=\mathrm{CC}=\mathrm{C}(\mathrm{C}=\mathrm{C} 5) \mathrm{OC}\end{array}$ \\
\hline $\mathrm{H} 70$ & + & 3.097 & 3.216 & 69.495 & 3.194 & 81.309 & $\begin{array}{l}\mathrm{O}=\mathrm{C} 1 \mathrm{O}[\mathrm{C} @ @]([\mathrm{H}])(\mathrm{CN} 1 \mathrm{C}=2 \mathrm{C}=\mathrm{CC}(=\mathrm{CC}=2) \mathrm{C}(\mathrm{C})=\mathrm{O}) \mathrm{C}(=\mathrm{O} \\
) \mathrm{N}[\mathrm{C} @ @]([\mathrm{H}])(\mathrm{C} / \mathrm{C} 3=\mathrm{C} / \mathrm{C}=\mathrm{CC}=\mathrm{C} 3)[\mathrm{C} @ @](\mathrm{O})([\mathrm{H}]) \mathrm{CN}(\mathrm{C}[ \\
\mathrm{C} @ @] 4([\mathrm{H}]) \mathrm{CC} 4)[\mathrm{S}](=\mathrm{O})(=\mathrm{O}) \mathrm{C} 5=\mathrm{CC}=\mathrm{C}(\mathrm{C}=\mathrm{C} 5) \mathrm{OC}\end{array}$ \\
\hline H71 & + & 0.622 & 0.950 & 20.533 & 0.827 & 21.052 & $\begin{array}{l}\mathrm{O}=\mathrm{C} 1 \mathrm{O}[\mathrm{C} @ @]([\mathrm{H}])(\mathrm{CN} 1 \mathrm{C}=2 \mathrm{C}=\mathrm{CC}=\mathrm{CC}=2) \mathrm{C}(=\mathrm{O}) \mathrm{N}[\mathrm{C} @ @) \\
]([\mathrm{H}])(\mathrm{C} / \mathrm{C} 3=\mathrm{C} / \mathrm{C}=\mathrm{CC}=\mathrm{C} 3)[\mathrm{C} @ @](\mathrm{O})([\mathrm{H}]) \mathrm{CN}(\mathrm{C} / \mathrm{C} 4=\mathrm{C} / \mathrm{C}= \\
\mathrm{C}[\mathrm{S}] 4)[\mathrm{S}](=\mathrm{O})(=\mathrm{O}) \mathrm{C} 5=\mathrm{CC}=\mathrm{CC}(=\mathrm{C} 5) \mathrm{OC}\end{array}$ \\
\hline $\mathrm{H} 73$ & + & 1.530 & 1.296 & 28.009 & 1.298 & 33.044 & $\begin{array}{l}\mathrm{O}=\mathrm{C} 1 \mathrm{O}[\mathrm{C} @ @]([\mathrm{H}])(\mathrm{CN} 1 \mathrm{C}=2 \mathrm{C}=\mathrm{CC}(=\mathrm{CC}=2) \mathrm{C}(=\mathrm{O}) \mathrm{C}) \mathrm{C}(=\mathrm{O} \\
) \mathrm{N}[\mathrm{C} @ @]([\mathrm{H}])(\mathrm{C} / \mathrm{C} 3=\mathrm{C} / \mathrm{C}=\mathrm{CC}=\mathrm{C} 3)[\mathrm{C} @ @](\mathrm{O})([\mathrm{H}]) \mathrm{CN}(\mathrm{C} / \\
\mathrm{C} 4=\mathrm{C} / \mathrm{C}=\mathrm{C}[\mathrm{S}] 4)[\mathrm{S}](=\mathrm{O})(=\mathrm{O}) \mathrm{C} 5=\mathrm{CC}=\mathrm{CC}(=\mathrm{C} 5) \mathrm{OC}\end{array}$ \\
\hline H76 & + & 0.775 & 1.017 & 21.975 & 1.171 & 29.819 & $\begin{array}{l}\mathrm{O}=\mathrm{C} 1 \mathrm{O}[\mathrm{C} @ @]([\mathrm{H}])(\mathrm{CN} 1 \mathrm{C}=2 \mathrm{C}=\mathrm{CC}(=\mathrm{CC}=2) \mathrm{C}(=\mathrm{O}) \mathrm{C}) \mathrm{C}(=\mathrm{O} \\
) \mathrm{N}[\mathrm{C} @ @]([\mathrm{H}])(\mathrm{C} / \mathrm{C} 3=\mathrm{C} / \mathrm{C}=\mathrm{CC}=\mathrm{C} 3)[\mathrm{C} @ @](\mathrm{O})([\mathrm{H}]) \mathrm{CN}(\mathrm{C} / \\
\mathrm{C} 4=\mathrm{C} / \mathrm{C}=\mathrm{C}[\mathrm{S}] 4)[\mathrm{S}](=\mathrm{O})(=\mathrm{O}) \mathrm{C} 5=\mathrm{CC}([\mathrm{F}])=\mathrm{C}([\mathrm{F}]) \mathrm{C}=\mathrm{C} 5[\mathrm{~F}]\end{array}$ \\
\hline H77 & + & 1.377 & 1.043 & 22.541 & 1.024 & 26.075 & $\begin{array}{l}\mathrm{O}=\mathrm{C} 1 \mathrm{O}[\mathrm{C} @ @]([\mathrm{H}])(\mathrm{CN} 1 \mathrm{C}=2 \mathrm{C}=\mathrm{CC}=\mathrm{CC}=2) \mathrm{C}(=\mathrm{O}) \mathrm{N}[\mathrm{C} @ @) \\
]([\mathrm{H}])(\mathrm{C} / \mathrm{C} 3=\mathrm{C} / \mathrm{C}=\mathrm{CC}=\mathrm{C} 3)[\mathrm{C} @ @](\mathrm{O})([\mathrm{H}]) \mathrm{CN}(\mathrm{C}[\mathrm{C} @ @] 4([ \\
\mathrm{H}]) \mathrm{CCCO})[\mathrm{S}](=\mathrm{O})(=\mathrm{O}) \mathrm{C} 5=\mathrm{CC}=\mathrm{CC}(=\mathrm{C} 5) \mathrm{OC}\end{array}$ \\
\hline $\mathrm{H} 78$ & + & 0.824 & 1.123 & 24.260 & 1.191 & 30.322 & $\begin{array}{l}\mathrm{O}=\mathrm{C} 1 \mathrm{O}[\mathrm{C} @ @]([\mathrm{H}])(\mathrm{CN} 1 \mathrm{C}=2 \mathrm{C}=\mathrm{C} / \mathrm{C}=\mathrm{C}(/[\mathrm{F}]) \mathrm{C}=2) \mathrm{C}(=\mathrm{O}) \mathrm{N}[ \\
\mathrm{C} @ @]([\mathrm{H}])(\mathrm{C} / \mathrm{C} 3=\mathrm{C} / \mathrm{C}=\mathrm{CC}=\mathrm{C} 3)[\mathrm{C} @ @](\mathrm{O})([\mathrm{H}]) \mathrm{CN}(\mathrm{C}[\mathrm{C} @ \\
@] 4([\mathrm{H}]) \mathrm{CCCO} 4)[\mathrm{S}](=\mathrm{O})(=\mathrm{O}) \mathrm{C} 5=\mathrm{CC}=\mathrm{CC}(=\mathrm{C} 5) \mathrm{OC}\end{array}$ \\
\hline H79 & + & 3.686 & 4.130 & 89.235 & 4.027 & 102.529 & $\begin{array}{l}\mathrm{O}=\mathrm{C} 1 \mathrm{O}[\mathrm{C} @ @]([\mathrm{H}])(\mathrm{CN} 1 \mathrm{C}=2 \mathrm{C}=\mathrm{CC}=\mathrm{CC}=2) \mathrm{C}(=\mathrm{O}) \mathrm{N}[\mathrm{C} @ @ \\
]([\mathrm{H}])(\mathrm{C} / \mathrm{C} 3=\mathrm{C} / \mathrm{C}=\mathrm{CC}=\mathrm{C} 3)[\mathrm{C} @ @](\mathrm{O})([\mathrm{H}]) \mathrm{CN}(\mathrm{CC}(\mathrm{C})(\mathrm{C})[\mathrm{H}] \\
)[\mathrm{S}](=\mathrm{O})(=\mathrm{O}) \mathrm{C} 5=\mathrm{CC}=\mathrm{C} 4 \mathrm{OCOC} 4=\mathrm{C} 5\end{array}$ \\
\hline H80 & + & 4.481 & 4.373 & 94.485 & 4.264 & 108.569 & $\begin{array}{l}\mathrm{O}=\mathrm{C} 1 \mathrm{O}[\mathrm{C} @ @]([\mathrm{H}])(\mathrm{CN} 1 \mathrm{C}=2 \mathrm{C}=\mathrm{CC}=\mathrm{CC}=2) \mathrm{C}(=\mathrm{O}) \mathrm{N}[\mathrm{C} @ @ \\
]([\mathrm{H}])(\mathrm{C} / \mathrm{C} 3=\mathrm{C} / \mathrm{C}=\mathrm{CC}=\mathrm{C} 3)[\mathrm{C} @(\mathrm{O})([\mathrm{H}]) \mathrm{CN}(\mathrm{CC}(\mathrm{C})(\mathrm{C})[\mathrm{H}] \\
)[\mathrm{S}](=\mathrm{O})(=\mathrm{O}) \mathrm{C} 5=\mathrm{CC}=\mathrm{C} 4 \mathrm{~N}=\mathrm{C}[\mathrm{S}] \mathrm{C} 4=\mathrm{C} 5\end{array}$ \\
\hline H83 & + & 2.248 & 2.568 & 55.485 & 2.604 & 66.287 & $\begin{array}{l}\mathrm{O}=\mathrm{C} 1 \mathrm{O}[\mathrm{C} @ @]([\mathrm{H}])(\mathrm{CN} 1 \mathrm{C}=2 \mathrm{C}=\mathrm{CC}=\mathrm{CC}=2 \mathrm{O}) \mathrm{C}(=\mathrm{O}) \mathrm{N}[\mathrm{C} @ \\
@]([\mathrm{H}])(\mathrm{C} / \mathrm{C} 3=\mathrm{C} / \mathrm{C}=\mathrm{CC}=\mathrm{C} 3)[\mathrm{C} @ @](\mathrm{O})([\mathrm{H}]) \mathrm{CN}(\mathrm{CC}(\mathrm{C})(\mathrm{C})[ \\
\mathrm{H}])[\mathrm{S}](=\mathrm{O})(=\mathrm{O}) \mathrm{C} 4=\mathrm{CC}=\mathrm{C}(\mathrm{C}=\mathrm{C} 4) \mathrm{OC}\end{array}$ \\
\hline H84 & + & 2.833 & 2.696 & 58.250 & 2.721 & 69.286 & $\begin{array}{l}\mathrm{O}=\mathrm{C} 1 \mathrm{O}[\mathrm{C} @ @]([\mathrm{H}])(\mathrm{CN} 1 \mathrm{C}=2 \mathrm{C}=\mathrm{CC}=\mathrm{CC}=2 \mathrm{O}) \mathrm{C}(=\mathrm{O}) \mathrm{N}[\mathrm{C} @ \\
@]([\mathrm{H}])(\mathrm{C} / \mathrm{C} 3=\mathrm{C} / \mathrm{C}=\mathrm{CC}=\mathrm{C} 3)[\mathrm{C} @ @](\mathrm{O})([\mathrm{H}]) \mathrm{CN}(\mathrm{CC}(\mathrm{C})(\mathrm{C})[\end{array}$ \\
\hline
\end{tabular}




\begin{tabular}{|c|c|c|c|c|c|c|c|}
\hline & & & & & & & $\mathrm{H}])[\mathrm{S}](=\mathrm{O})(=\mathrm{O}) \mathrm{C} 5=\mathrm{CC}=\mathrm{C} 4 \mathrm{OCOC} 4=\mathrm{C} 5$ \\
\hline $\mathrm{H} 87$ & + & 3.848 & 3.978 & 85.958 & 3.989 & 101.556 & $\begin{array}{l}\mathrm{O}=\mathrm{C} 1 \mathrm{O}[\mathrm{C} @ @]([\mathrm{H}])(\mathrm{CN} 1 \mathrm{C}=2 \mathrm{C}=\mathrm{CC}=\mathrm{CC}=2 \mathrm{C}([\mathrm{F}])([\mathrm{F}])[\mathrm{F}]) \mathrm{C} \\
(=\mathrm{O}) \mathrm{N}[\mathrm{C} @ @]([\mathrm{H}])(\mathrm{C} / \mathrm{C} 3=\mathrm{C} / \mathrm{C}=\mathrm{CC}=\mathrm{C} 3)[\mathrm{C} @ @](\mathrm{O})([\mathrm{H}]) \mathrm{CN} \\
(\mathrm{CC}(\mathrm{C})(\mathrm{C})[\mathrm{H}])[\mathrm{S}](=\mathrm{O})(=\mathrm{O}) \mathrm{C} 5=\mathrm{CC}=\mathrm{C} 4 \mathrm{OCOC} 4=\mathrm{C} 5\end{array}$ \\
\hline H89 & + & 3.629 & 3.214 & 69.446 & 3.213 & 81.797 & $\begin{array}{l}\mathrm{O}=\mathrm{C} 1 \mathrm{O}[\mathrm{C} @ @]([\mathrm{H}])(\mathrm{CN} 1 \mathrm{C}=2 \mathrm{C}=\mathrm{CC}=\mathrm{CC}=2 \mathrm{C}([\mathrm{F}])([\mathrm{F}])[\mathrm{F}]) \mathrm{C} \\
(=\mathrm{O}) \mathrm{N}[\mathrm{C} @ @]([\mathrm{H}])(\mathrm{C} / \mathrm{C} 3=\mathrm{C} / \mathrm{C}=\mathrm{CC}=\mathrm{C} 3)[\mathrm{C} @ @](\mathrm{O})([\mathrm{H}]) \mathrm{CN} \\
(\mathrm{CC}(\mathrm{C})(\mathrm{C})[\mathrm{H}])[\mathrm{S}](=\mathrm{O})(=\mathrm{O}) \mathrm{C} 4=\mathrm{CC}=\mathrm{C}(\mathrm{C}=\mathrm{C} 4) \mathrm{CO}\end{array}$ \\
\hline H91 & + & 4.201 & 3.655 & 78.968 & 3.675 & 93.571 & $\begin{array}{l}\mathrm{O}=\mathrm{C} 1 \mathrm{O}[\mathrm{C} @ @]([\mathrm{H}])(\mathrm{CN} 1 \mathrm{C}=2 \mathrm{C}=\mathrm{CC}([\mathrm{F}])=\mathrm{CC}=2[\mathrm{~F}]) \mathrm{C}(=\mathrm{O}) \mathrm{N} \\
{[\mathrm{C} @ @]([\mathrm{H}])(\mathrm{C} / \mathrm{C} 3=\mathrm{C} / \mathrm{C}=\mathrm{CC}=\mathrm{C} 3)[\mathrm{C} @ @](\mathrm{O})([\mathrm{H}]) \mathrm{CN}(\mathrm{CC}(\mathrm{C}} \\
)(\mathrm{C})[\mathrm{H}])[\mathrm{S}](=\mathrm{O})(=\mathrm{O}) \mathrm{C} 4=\mathrm{CC}=\mathrm{C}(\mathrm{C}=\mathrm{C} 4) \mathrm{OC}\end{array}$ \\
\hline H92 & + & 3.460 & 3.783 & 81.732 & 3.793 & 96.570 & $\begin{array}{l}\mathrm{O}=\mathrm{C} 1 \mathrm{O}[\mathrm{C} @ @]([\mathrm{H}])(\mathrm{CN} 1 \mathrm{C}=2 \mathrm{C}=\mathrm{CC}([\mathrm{F}])=\mathrm{CC}=2[\mathrm{~F}]) \mathrm{C}(=\mathrm{O}) \mathrm{N} \\
{[\mathrm{C} @ @]([\mathrm{H}])(\mathrm{C} / \mathrm{C} 3=\mathrm{C} / \mathrm{C}=\mathrm{CC}=\mathrm{C} 3)[\mathrm{C} @ @](\mathrm{O})([\mathrm{H}]) \mathrm{CN}(\mathrm{CC}(\mathrm{C}} \\
)(\mathrm{C})[\mathrm{H}])[\mathrm{S}](=\mathrm{O})(=\mathrm{O}) \mathrm{C} 5=\mathrm{CC}=\mathrm{C} 4 \mathrm{OCOC} 4=\mathrm{C} 5\end{array}$ \\
\hline H93 & + & 3.824 & 4.026 & 86.983 & 4.030 & 102.610 & $\begin{array}{l}\mathrm{O}=\mathrm{C} 1 \mathrm{O}[\mathrm{C} @ @]([\mathrm{H}])(\mathrm{CN} 1 \mathrm{C}=2 \mathrm{C}=\mathrm{CC}([\mathrm{F}])=\mathrm{CC}=2[\mathrm{~F}]) \mathrm{C}(=\mathrm{O}) \mathrm{N} \\
{[\mathrm{C} @ @]([\mathrm{H}])(\mathrm{C} / \mathrm{C} 3=\mathrm{C} / \mathrm{C}=\mathrm{CC}=\mathrm{C} 3)[\mathrm{C} @ @](\mathrm{O})([\mathrm{H}]) \mathrm{CN}(\mathrm{CC}(\mathrm{C}} \\
)(\mathrm{C})[\mathrm{H}])[\mathrm{S}](=\mathrm{O})(=\mathrm{O}) \mathrm{C} 5=\mathrm{CC}=\mathrm{C} 4 \mathrm{~N}=\mathrm{C}[\mathrm{S}] \mathrm{C} 4=\mathrm{C} 5\end{array}$ \\
\hline H95 & + & 3.349 & 3.666 & 79.205 & 3.517 & 89.534 & $\begin{array}{l}\mathrm{O}=\mathrm{C} 1 \mathrm{O}[\mathrm{C} @ @]([\mathrm{H}])(\mathrm{CN} 1 \mathrm{C}=2 \mathrm{C}=\mathrm{CC}([\mathrm{F}])=\mathrm{CC}=2) \mathrm{C}(=\mathrm{O}) \mathrm{N}[\mathrm{C} \\
@ @]([\mathrm{H}])(\mathrm{C} / \mathrm{C} 3=\mathrm{C} / \mathrm{C}=\mathrm{CC}=\mathrm{C} 3)[\mathrm{C} @ @](\mathrm{O})([\mathrm{H}]) \mathrm{CN}(\mathrm{CC}(\mathrm{C})( \\
\mathrm{C})[\mathrm{H}])[\mathrm{S}](=\mathrm{O})(=\mathrm{O}) \mathrm{C} 4=\mathrm{C} / \mathrm{C}=\mathrm{C}(/ \mathrm{N}) \mathrm{C}=\mathrm{C} 4\end{array}$ \\
\hline H98 & + & 3.876 & 4.477 & 96.729 & 4.353 & 110.837 & $\begin{array}{l}\mathrm{O}=\mathrm{C} 1 \mathrm{O}[\mathrm{C} @ @]([\mathrm{H}])(\mathrm{CN} 1 \mathrm{C}=2 \mathrm{C}=\mathrm{CC}([\mathrm{F}])=\mathrm{CC}=2) \mathrm{C}(=\mathrm{O}) \mathrm{N}[\mathrm{C} \\
@ @]([\mathrm{H}])(\mathrm{C} / \mathrm{C} 3=\mathrm{C} / \mathrm{C}=\mathrm{CC}=\mathrm{C} 3)[\mathrm{C} @ @](\mathrm{O})([\mathrm{H}]) \mathrm{CN}(\mathrm{CC}(\mathrm{C})( \\
\mathrm{C})[\mathrm{H}])[\mathrm{S}](=\mathrm{O})(=\mathrm{O}) \mathrm{C} 5=\mathrm{CC}=\mathrm{C} 4 \mathrm{~N}=\mathrm{C}[\mathrm{S}] \mathrm{C} 4=\mathrm{C} 5\end{array}$ \\
\hline H100 & + & 4.137 & 4.719 & 101.961 & 4.735 & 120.561 & $\begin{array}{l}\mathrm{O}=\mathrm{C} 1 \mathrm{O}[\mathrm{C} @ @]([\mathrm{H}])(\mathrm{CN} 1 \mathrm{C}=2 \mathrm{C}=\mathrm{CC}(=\mathrm{CC}=2) \mathrm{C}(\mathrm{C})=\mathrm{O}) \mathrm{C}(=\mathrm{O} \\
) \mathrm{N}[\mathrm{C} @ @]([\mathrm{H}])(\mathrm{C} / \mathrm{C} 3=\mathrm{C} / \mathrm{C}=\mathrm{CC}=\mathrm{C} 3)[\mathrm{C} @ @](\mathrm{O})([\mathrm{H}]) \mathrm{CN}(\mathrm{CC} \\
(\mathrm{C})(\mathrm{C})[\mathrm{H}])[\mathrm{S}](=\mathrm{O})(=\mathrm{O}) \mathrm{C} 5=\mathrm{CC}=\mathrm{C} 4 \mathrm{~N}=\mathrm{C}[\mathrm{S}] \mathrm{C} 4=\mathrm{C} 5\end{array}$ \\
\hline H101 & + & 3.499 & 3.712 & 80.198 & 3.722 & 94.762 & $\begin{array}{l}\mathrm{O}=\mathrm{C} 1 \mathrm{O}[\mathrm{C} @ @]([\mathrm{H}])(\mathrm{CN} 1 \mathrm{C}=2 \mathrm{C}=\mathrm{CC}(=\mathrm{CC}=2) \mathrm{C}(\mathrm{C})=\mathrm{O}) \mathrm{C}(=\mathrm{O} \\
) \mathrm{N}[\mathrm{C} @ @]([\mathrm{H}])(\mathrm{C} / \mathrm{C} 3=\mathrm{C} / \mathrm{C}=\mathrm{CC}=\mathrm{C} 3)[\mathrm{C} @ @](\mathrm{O})([\mathrm{H}]) \mathrm{CN}(\mathrm{CC} \\
(\mathrm{C})(\mathrm{C})[\mathrm{H}])[\mathrm{S}](=\mathrm{O})(=\mathrm{O}) \mathrm{C} 4=\mathrm{CC}=\mathrm{C}(\mathrm{C}=\mathrm{C} 4) \mathrm{CO}\end{array}$ \\
\hline H102 & + & 4.097 & 4.453 & 96.205 & 4.431 & 112.816 & $\begin{array}{l}\mathrm{O}=\mathrm{C} 1 \mathrm{O}[\mathrm{C} @ @]([\mathrm{H}])(\mathrm{CN} 1 \mathrm{C}=2 \mathrm{C}=\mathrm{C} / \mathrm{C}=\mathrm{C}(/[\mathrm{F}]) \mathrm{C}=2) \mathrm{C}(=\mathrm{O}) \mathrm{N}[ \\
\mathrm{C} @ @]([\mathrm{H}])(\mathrm{C} / \mathrm{C} 3=\mathrm{C} / \mathrm{C}=\mathrm{CC}=\mathrm{C} 3)[\mathrm{C} @ @](\mathrm{O})([\mathrm{H}]) \mathrm{CN}(\mathrm{CC}(\mathrm{C}) \\
(\mathrm{C})[\mathrm{H}])[\mathrm{S}](=\mathrm{O})(=\mathrm{O}) \mathrm{C} 5=\mathrm{CC}=\mathrm{C} 4 \mathrm{~N}=\mathrm{C}[\mathrm{S}] \mathrm{C} 4=\mathrm{C} 5\end{array}$ \\
\hline H104 & + & 3.635 & 4.603 & 99.445 & 4.628 & 117.839 & $\begin{array}{l}\mathrm{O}=\mathrm{C} 1 \mathrm{O}[\mathrm{C} @ @]([\mathrm{H}])(\mathrm{CN} 1 \mathrm{C}=2 \mathrm{C}=\mathrm{CC}([\mathrm{F}])=\mathrm{C}([\mathrm{F}]) \mathrm{C}=2) \mathrm{C}(=\mathrm{O}) \\
\mathrm{N}[\mathrm{C} @ @]([\mathrm{H}])(\mathrm{C} / \mathrm{C} 3=\mathrm{C} / \mathrm{C}=\mathrm{CC}=\mathrm{C} 3)[\mathrm{C} @ @](\mathrm{O})([\mathrm{H}]) \mathrm{CN}(\mathrm{CC}( \\
\mathrm{C})(\mathrm{C})[\mathrm{H}])[\mathrm{S}](=\mathrm{O})(=\mathrm{O}) \mathrm{C} 5=\mathrm{CC}=\mathrm{C} 4 \mathrm{~N}=\mathrm{C}[\mathrm{S}] \mathrm{C} 4=\mathrm{C} 5\end{array}$ \\
\hline H105 & + & 3.481 & 3.595 & 77.682 & 3.615 & 92.040 & $\begin{array}{l}\mathrm{O}=\mathrm{C} 1 \mathrm{O}[\mathrm{CC} @]]([\mathrm{H}])(\mathrm{CN} 1 \mathrm{C}=2 \mathrm{C}=\mathrm{CC}([\mathrm{F}])=\mathrm{C}([\mathrm{F}]) \mathrm{C}=2) \mathrm{C}(=\mathrm{O}) \\
\mathrm{N}[\mathrm{C} @ @]([\mathrm{H}])(\mathrm{C} / \mathrm{C} 3=\mathrm{C} / \mathrm{C}=\mathrm{CC}=\mathrm{C} 3)[\mathrm{C} @ @](\mathrm{O})([\mathrm{H}]) \mathrm{CN}(\mathrm{CC}( \\
\mathrm{C})(\mathrm{C})[\mathrm{H}])[\mathrm{S}](=\mathrm{O})(=\mathrm{O}) \mathrm{C} 4=\mathrm{CC}=\mathrm{C}(\mathrm{C}=\mathrm{C} 4) \mathrm{CO}\end{array}$ \\
\hline H106 & + & 4.796 & 4.266 & 92.174 & 4.265 & 108.583 & $\begin{array}{l}\mathrm{O}=\mathrm{C} 1 \mathrm{O}[\mathrm{C} @ @]([\mathrm{H}])(\mathrm{CN} 1 \mathrm{C}=2 \mathrm{C}=\mathrm{CC}=\mathrm{C}(\mathrm{C}=2) \mathrm{C}([\mathrm{F}])([\mathrm{F}])[\mathrm{F}]) \\
\mathrm{C}(=\mathrm{O}) \mathrm{N}[\mathrm{C} @ @]([\mathrm{H}])(\mathrm{C} / \mathrm{C} 3=\mathrm{C} / \mathrm{C}=\mathrm{CC}=\mathrm{C} 3)[\mathrm{C} @ @](\mathrm{O})([\mathrm{H}]) \mathrm{C} \\
\mathrm{N}(\mathrm{CC}(\mathrm{C})(\mathrm{C})[\mathrm{H}])[\mathrm{S}](=\mathrm{O})(=\mathrm{O}) \mathrm{C} 5=\mathrm{CC}=\mathrm{C} 4 \mathrm{~N}=\mathrm{C}[\mathrm{S}] \mathrm{C} 4=\mathrm{C} 5\end{array}$ \\
\hline H107 & + & 3.708 & 3.259 & 70.411 & 3.252 & 82.784 & $\begin{array}{l}\mathrm{O}=\mathrm{C} 1 \mathrm{O}[\mathrm{C} @ @]([\mathrm{H}])(\mathrm{CN} 1 \mathrm{C}=2 \mathrm{C}=\mathrm{CC}=\mathrm{C}(\mathrm{C}=2) \mathrm{C}([\mathrm{F}])([\mathrm{F}])[\mathrm{F}]) \\
\mathrm{C}(=\mathrm{O}) \mathrm{N}[\mathrm{C} @ @]([\mathrm{H}])(\mathrm{C} / \mathrm{C} 3=\mathrm{C} / \mathrm{C}=\mathrm{CC}=\mathrm{C} 3)[\mathrm{C} @ @](\mathrm{O})([\mathrm{H}]) \mathrm{C} \\
\mathrm{N}(\mathrm{CC}(\mathrm{C})(\mathrm{C})[\mathrm{H}])[\mathrm{S}](=\mathrm{O})(=\mathrm{O}) \mathrm{C} 4=\mathrm{CC}=\mathrm{C}(\mathrm{C}=\mathrm{C} 4) \mathrm{CO}\end{array}$ \\
\hline H109 & + & 3.886 & 3.942 & 85.172 & 3.989 & 101.556 & $\begin{array}{l}\mathrm{O}=\mathrm{C} 1 \mathrm{O}[\mathrm{C} @ @]([\mathrm{H}])(\mathrm{CN} 1 \mathrm{C}=2 \mathrm{C}=\mathrm{CC}=\mathrm{C}(\mathrm{C}=2) \mathrm{OC}([\mathrm{F}])([\mathrm{F}])[\mathrm{F} \\
]) \mathrm{C}(=\mathrm{O}) \mathrm{N}[\mathrm{C} @ @]([\mathrm{H}])(\mathrm{C} / \mathrm{C} 3=\mathrm{C} / \mathrm{C}=\mathrm{CC}=\mathrm{C} 3)[\mathrm{C} @ @](\mathrm{O})([\mathrm{H}]) \\
\mathrm{CN}(\mathrm{CC}(\mathrm{C})(\mathrm{C})[\mathrm{H}])[\mathrm{S}](=\mathrm{O})(=\mathrm{O}) \mathrm{C} 4=\mathrm{CC}=\mathrm{C}(\mathrm{C}=\mathrm{C} 4) \mathrm{OC}\end{array}$ \\
\hline H111 & + & 4.585 & 4.313 & 93.187 & 4.344 & 110.595 & $\begin{array}{l}\mathrm{O}=\mathrm{C} 1 \mathrm{O}[\mathrm{C} @ @]([\mathrm{H}])(\mathrm{CN} 1 \mathrm{C}=2 \mathrm{C}=\mathrm{CC}=\mathrm{C}(\mathrm{C}=2) \mathrm{OC}([\mathrm{F}])([\mathrm{F}])[\mathrm{F} \\
]) \mathrm{C}(=\mathrm{O}) \mathrm{N}[\mathrm{C} @ @]([\mathrm{H}])(\mathrm{C} / \mathrm{C} 3=\mathrm{C} / \mathrm{C}=\mathrm{CC}=\mathrm{C} 3)[\mathrm{C} @ @](\mathrm{O})([\mathrm{H}]) \\
\mathrm{CN}(\mathrm{CC}(\mathrm{C})(\mathrm{C})[\mathrm{H}])[\mathrm{S}](=\mathrm{O})(=\mathrm{O}) \mathrm{C} 5=\mathrm{CC}=\mathrm{C} 4 \mathrm{~N}=\mathrm{C}[\mathrm{S}] \mathrm{C} 4=\mathrm{C} 5\end{array}$ \\
\hline
\end{tabular}




\begin{tabular}{|c|c|c|c|c|c|c|c|}
\hline H113 & + & 4.824 & 5.100 & 110.193 & 5.108 & 130.049 & $\begin{array}{l}\mathrm{O}=\mathrm{C} 1 \mathrm{O}[\mathrm{C} @ @]([\mathrm{H}])(\mathrm{CN} 1 \mathrm{C}=2 \mathrm{C}=\mathrm{CC}=\mathrm{C}(\mathrm{C}=2) \mathrm{C}(\mathrm{C})=\mathrm{O}) \mathrm{C}(=\mathrm{O} \\
) \mathrm{N}[\mathrm{C} @ @]([\mathrm{H}])(\mathrm{C} / \mathrm{C} 3=\mathrm{C} / \mathrm{C}=\mathrm{CC}=\mathrm{C} 3)[\mathrm{C} @ @](\mathrm{O})([\mathrm{H}]) \mathrm{CN}(\mathrm{CC} \\
(\mathrm{C})(\mathrm{C})[\mathrm{H}])[\mathrm{S}](=\mathrm{O})(=\mathrm{O}) \mathrm{C} 5=\mathrm{CC}=\mathrm{C} 4 \mathrm{~N}=\mathrm{C}[\mathrm{S}] \mathrm{C} 4=\mathrm{C} 5\end{array}$ \\
\hline H114 & + & 3.627 & 4.093 & 88.430 & 4.095 & 104.250 & $\begin{array}{l}\mathrm{O}=\mathrm{C} 1 \mathrm{O}[\mathrm{C} @ @]([\mathrm{H}])(\mathrm{CN} 1 \mathrm{C}=2 \mathrm{C}=\mathrm{CC}=\mathrm{C}(\mathrm{C}=2) \mathrm{C}(\mathrm{C})=\mathrm{O}) \mathrm{C}(=\mathrm{O} \\
) \mathrm{N}[\mathrm{C} @ @]([\mathrm{H}])(\mathrm{C} / \mathrm{C} 3=\mathrm{C} / \mathrm{C}=\mathrm{CC}=\mathrm{C} 3)[\mathrm{C} @ @](\mathrm{O})([\mathrm{H}]) \mathrm{CN}(\mathrm{CC} \\
(\mathrm{C})(\mathrm{C})[\mathrm{H}])[\mathrm{S}](=\mathrm{O})(=\mathrm{O}) \mathrm{C} 4=\mathrm{CC}=\mathrm{C}(\mathrm{C}=\mathrm{C} 4) \mathrm{CO}\end{array}$ \\
\hline H116 & + & 4.602 & 4.729 & 102.170 & 4.734 & 120.519 & $\begin{array}{l}\mathrm{O}=\mathrm{C} 1 \mathrm{O}[\mathrm{C} @ @]([\mathrm{H}])(\mathrm{CN} 1 \mathrm{C}=2 \mathrm{C}=\mathrm{CC}=\mathrm{C}(\mathrm{C}=2)[\mathrm{S}](\mathrm{C})(=\mathrm{O})=\mathrm{O}) \\
\mathrm{C}(=\mathrm{O}) \mathrm{N}[\mathrm{C} @ @]([\mathrm{H}])(\mathrm{C} / \mathrm{C} 3=\mathrm{C} / \mathrm{C}=\mathrm{CC}=\mathrm{C} 3)[\mathrm{C} @ @](\mathrm{O})([\mathrm{H}]) \mathrm{C} \\
\mathrm{N}(\mathrm{CC}(\mathrm{C})(\mathrm{C})[\mathrm{H}])[\mathrm{S}](=\mathrm{O})(=\mathrm{O}) \mathrm{C} 5=\mathrm{CC}=\mathrm{C} 4 \mathrm{OCOC} 4=\mathrm{C} 5\end{array}$ \\
\hline H117 & + & 5.523 & 4.972 & 107.421 & 4.971 & 126.560 & $\begin{array}{l}\mathrm{O}=\mathrm{C} 1 \mathrm{O}[\mathrm{C} @ @]([\mathrm{H}])(\mathrm{CN} 1 \mathrm{C}=2 \mathrm{C}=\mathrm{CC}=\mathrm{C}(\mathrm{C}=2)[\mathrm{S}](\mathrm{C})(=\mathrm{O})=\mathrm{O}) \\
\mathrm{C}(=\mathrm{O}) \mathrm{N}[\mathrm{C} @ @]([\mathrm{H}])(\mathrm{C} / \mathrm{C} 3=\mathrm{C} / \mathrm{C}=\mathrm{CC}=\mathrm{C} 3)[\mathrm{C} @ @](\mathrm{O})([\mathrm{H}]) \mathrm{C} \\
\mathrm{N}(\mathrm{CC}(\mathrm{C})(\mathrm{C})[\mathrm{H}])[\mathrm{S}](=\mathrm{O})(=\mathrm{O}) \mathrm{C} 5=\mathrm{CC}=\mathrm{C} 4 \mathrm{~N}=\mathrm{C}[\mathrm{S}] \mathrm{C} 4=\mathrm{C} 5\end{array}$ \\
\hline H118 & + & 3.866 & 4.219 & 91.168 & 4.244 & 108.048 & $\begin{array}{l}\mathrm{O}=\mathrm{C} 1 \mathrm{O}[\mathrm{C} @ @]([\mathrm{H}])(\mathrm{CN} 1 \mathrm{C}=2 \mathrm{C}=\mathrm{CC}=\mathrm{C}(\mathrm{C}=2)[\mathrm{N}+](=\mathrm{O})[\mathrm{O}- \\
]) \mathrm{C}(=\mathrm{O}) \mathrm{N}[\mathrm{C} @ @]([\mathrm{H}])(\mathrm{C} / \mathrm{C} 3=\mathrm{C} / \mathrm{C}=\mathrm{CC}=\mathrm{C} 3)[\mathrm{C} @ @](\mathrm{O})([\mathrm{H}]) \\
\mathrm{CN}(\mathrm{CC}(\mathrm{C})(\mathrm{C})[\mathrm{H}])[\mathrm{S}](=\mathrm{O})(=\mathrm{O}) \mathrm{C} 4=\mathrm{CC}=\mathrm{C}(\mathrm{C}=\mathrm{C} 4) \mathrm{OC}\end{array}$ \\
\hline H119 & + & 3.932 & 4.347 & 93.933 & 4.362 & 111.047 & $\begin{array}{l}\mathrm{O}=\mathrm{C} 1 \mathrm{O}[\mathrm{C} @ @]([\mathrm{H}])(\mathrm{CN} 1 \mathrm{C}=2 \mathrm{C}=\mathrm{CC}=\mathrm{C}(\mathrm{C}=2)[\mathrm{N}+](=\mathrm{O})[\mathrm{O}- \\
]) \mathrm{C}(=\mathrm{O}) \mathrm{N}[\mathrm{C} @ @]([\mathrm{H}])(\mathrm{C} / \mathrm{C} 3=\mathrm{C} / \mathrm{C}=\mathrm{CC}=\mathrm{C} 3)[\mathrm{C} @ @](\mathrm{O})([\mathrm{H}]) \\
\mathrm{CN}(\mathrm{CC}(\mathrm{C})(\mathrm{C})[\mathrm{H}])[\mathrm{S}](=\mathrm{O})(=\mathrm{O}) \mathrm{C} 5=\mathrm{CC}=\mathrm{C} 4 \mathrm{OCOC} 4=\mathrm{C} 5\end{array}$ \\
\hline H121 & + & 4.699 & 4.672 & 100.937 & 4.812 & 122.517 & $\begin{array}{l}\mathrm{O}=\mathrm{C} 1 \mathrm{O}[\mathrm{C} @ @]([\mathrm{H}])(\mathrm{CN} 1 \mathrm{C}=2 \mathrm{C}=\mathrm{C} / \mathrm{C}=\mathrm{C}(/ \mathrm{N}) \mathrm{C}=2) \mathrm{C}(=\mathrm{O}) \mathrm{N}[\mathrm{C} \\
@ @]([\mathrm{H}])(\mathrm{C} / \mathrm{C} 3=\mathrm{C} / \mathrm{C}=\mathrm{CC}=\mathrm{C} 3)[\mathrm{C} @ @](\mathrm{O})([\mathrm{H}]) \mathrm{CN}(\mathrm{CC}(\mathrm{C})( \\
\mathrm{C})[\mathrm{H}])[\mathrm{S}](=\mathrm{O})(=\mathrm{O}) \mathrm{C} 4=\mathrm{CC}=\mathrm{C}(\mathrm{C}=\mathrm{C} 4) \mathrm{OC}\end{array}$ \\
\hline H123 & + & 5.097 & 5.043 & 108.952 & 5.167 & 131.556 & $\begin{array}{l}\mathrm{O}=\mathrm{C} 1 \mathrm{O}[\mathrm{C} @ @]([\mathrm{H}])(\mathrm{CN} 1 \mathrm{C}=2 \mathrm{C}=\mathrm{C} / \mathrm{C}=\mathrm{C}(/ \mathrm{N}) \mathrm{C}=2) \mathrm{C}(=\mathrm{O}) \mathrm{N}[\mathrm{C} \\
@ @]([\mathrm{H}])(\mathrm{C} / \mathrm{C} 3=\mathrm{C} / \mathrm{C}=\mathrm{CC}=\mathrm{C} 3)[\mathrm{C} @ @](\mathrm{O})([\mathrm{H}]) \mathrm{CN}(\mathrm{CC}(\mathrm{C})( \\
\mathrm{C})[\mathrm{H}])[\mathrm{S}](=\mathrm{O})(=\mathrm{O}) \mathrm{C} 5=\mathrm{CC}=\mathrm{C} 4 \mathrm{~N}=\mathrm{C}[\mathrm{S}] \mathrm{C} 4=\mathrm{C} 5\end{array}$ \\
\hline H125 & + & 4.292 & 4.568 & 98.705 & 4.497 & 114.505 & $\begin{array}{l}\mathrm{O}=\mathrm{C} 1 \mathrm{O}[\mathrm{C} @ @]([\mathrm{H}])(\mathrm{CN} 1 \mathrm{C}=2 \mathrm{C}=\mathrm{CC}=\mathrm{C}(\mathrm{C}=2) \mathrm{NC}(=\mathrm{O}) \mathrm{C}) \mathrm{C}(= \\
\mathrm{O}) \mathrm{N}[\mathrm{C} @ @]([\mathrm{H}])(\mathrm{C} / \mathrm{C} 3=\mathrm{C} / \mathrm{C}=\mathrm{CC}=\mathrm{C} 3)[\mathrm{C} @ @](\mathrm{O})([\mathrm{H}]) \mathrm{CN}(\mathrm{C} \\
\mathrm{C}(\mathrm{C})(\mathrm{C})[\mathrm{H}])[\mathrm{S}](=\mathrm{O})(=\mathrm{O}) \mathrm{C} 5=\mathrm{CC}=\mathrm{C} 4 \mathrm{OCOC} 4=\mathrm{C} 5\end{array}$ \\
\hline H126 & + & 5.222 & 4.811 & 103.956 & 4.735 & 120.546 & $\begin{array}{l}\mathrm{O}=\mathrm{C} 1 \mathrm{O}[\mathrm{C} @ @]([\mathrm{H}])(\mathrm{CN} 1 \mathrm{C}=2 \mathrm{C}=\mathrm{CC}=\mathrm{C}(\mathrm{C}=2) \mathrm{NC}(=\mathrm{O}) \mathrm{C}) \mathrm{C}(= \\
\mathrm{O}) \mathrm{N}[\mathrm{C} @ @]([\mathrm{H}])(\mathrm{C} / \mathrm{C} 3=\mathrm{C} / \mathrm{C}=\mathrm{CC}=\mathrm{C} 3)[\mathrm{C} @ @](\mathrm{O})([\mathrm{H}]) \mathrm{CN}(\mathrm{C} \\
\mathrm{C}(\mathrm{C})(\mathrm{C})[\mathrm{H}])[\mathrm{S}](=\mathrm{O})(=\mathrm{O}) \mathrm{C} 5=\mathrm{CC}=\mathrm{C} 4 \mathrm{~N}=\mathrm{C}[\mathrm{S}] \mathrm{C} 4=\mathrm{C} 5\end{array}$ \\
\hline H129 & + & 4.585 & 4.140 & 89.461 & 4.195 & 106.805 & $\begin{array}{l}\mathrm{O}=\mathrm{C} 1 \mathrm{O}[\mathrm{C} @ @]([\mathrm{H}])(\mathrm{CN} 1 \mathrm{C}=2 \mathrm{C}=\mathrm{CC}=\mathrm{C}(\mathrm{C}=2) \mathrm{NC}(=\mathrm{O}) \mathrm{OC}) \mathrm{C}( \\
=\mathrm{O}) \mathrm{N}[\mathrm{C} @ @]([\mathrm{H}])(\mathrm{C} / \mathrm{C} 3=\mathrm{C} / \mathrm{C}=\mathrm{CC}=\mathrm{C} 3)[\mathrm{C} @ @](\mathrm{O})([\mathrm{H}]) \mathrm{CN}( \\
\mathrm{CC}(\mathrm{C})(\mathrm{C})[\mathrm{H}])[\mathrm{S}](=\mathrm{O})(=\mathrm{O}) \mathrm{C} 5=\mathrm{CC}=\mathrm{C} 4 \mathrm{~N}=\mathrm{C}[\mathrm{S}] \mathrm{C} 4=\mathrm{C} 5\end{array}$ \\
\hline H1 & - & 3.614 & 3.821 & 82.562 & 3.851 & 98.039 & $\begin{array}{l}\mathrm{O}=\mathrm{C}(\mathrm{OC}(\mathrm{C})(\mathrm{C}) \mathrm{C}) \mathrm{N}[\mathrm{C} @ @]([\mathrm{H}])(\mathrm{C} / \mathrm{C} 1=\mathrm{C} / \mathrm{C}=\mathrm{CC}=\mathrm{C} 1)[\mathrm{C} @]( \\
\mathrm{O})([\mathrm{H}]) \mathrm{CN}(\mathrm{C}[\mathrm{C} @ @](\mathrm{C})([\mathrm{H}]) \mathrm{CC})[\mathrm{S}](=\mathrm{O})(=\mathrm{O}) \mathrm{C} 3=\mathrm{CC}=\mathrm{C} 2 \mathrm{~N} \\
=\mathrm{C}[\mathrm{S}] \mathrm{C} 2=\mathrm{C} 3\end{array}$ \\
\hline $\mathrm{H} 7$ & - & 4.553 & 4.352 & 94.042 & 4.313 & 109.817 & $\begin{array}{l}\mathrm{O}=\mathrm{C}(\mathrm{OC}[\mathrm{C} @ @](\mathrm{C})([\mathrm{H}]) \mathrm{C}(=\mathrm{O}) \mathrm{OC}) \mathrm{N}[\mathrm{C} @ @]([\mathrm{H}])(\mathrm{C} / \mathrm{C} 1=\mathrm{C} / \\
\mathrm{C}=\mathrm{CC}=\mathrm{C} 1)[\mathrm{C} @](\mathrm{O})([\mathrm{H}]) \mathrm{CN}(\mathrm{C}[\mathrm{C} @ @](\mathrm{C})([\mathrm{H}]) \mathrm{CC})[\mathrm{S}](=\mathrm{O})( \\
=\mathrm{O}) \mathrm{C} 2=\mathrm{CC}=\mathrm{C}(\mathrm{C}=\mathrm{C} 2) \mathrm{OC}\end{array}$ \\
\hline H9 & - & 5.523 & 5.395 & 116.559 & 5.412 & 137.791 & $\begin{array}{l}\mathrm{O}=\mathrm{C}(\mathrm{OCCN} 1 \mathrm{CCOC} 1=\mathrm{O}) \mathrm{N}[\mathrm{C} @ @]([\mathrm{H}])(\mathrm{C} / \mathrm{C} 2=\mathrm{C} / \mathrm{C}=\mathrm{CC}=\mathrm{C} 2 \\
)[\mathrm{C} @](\mathrm{O})([\mathrm{H}]) \mathrm{CN}(\mathrm{C}[\mathrm{C} @ @](\mathrm{C})([\mathrm{H}]) \mathrm{CC})[\mathrm{S}](=\mathrm{O})(=\mathrm{O}) \mathrm{C} 3=\mathrm{C} \\
\mathrm{C}=\mathrm{C}(\mathrm{C}=\mathrm{C} 3) \mathrm{OC}\end{array}$ \\
\hline $\mathrm{H} 20$ & - & 0.915 & 1.530 & 33.062 & 1.525 & 38.832 & $\begin{array}{l}\mathrm{O}=\mathrm{C}(\mathrm{O}[\mathrm{C} @] 1([\mathrm{H}]) \mathrm{CCOC} 1=\mathrm{O}) \mathrm{N}[\mathrm{C} @ @]([\mathrm{H}])(\mathrm{C} / \mathrm{C} 2=\mathrm{C} / \mathrm{C}=\mathrm{C} \\
\mathrm{C}=\mathrm{C} 2)[\mathrm{C} @](\mathrm{O})([\mathrm{H}]) \mathrm{CN}(\mathrm{C}[\mathrm{C} @ @](\mathrm{C})([\mathrm{H}]) \mathrm{CC})[\mathrm{S}](=\mathrm{O})(=\mathrm{O}) \\
\mathrm{C} 4=\mathrm{CC}=\mathrm{C} 3 \mathrm{~N}=\mathrm{C}[\mathrm{S}] \mathrm{C} 3=\mathrm{C} 4\end{array}$ \\
\hline $\mathrm{H} 25$ & - & 2.529 & 2.652 & 57.311 & 2.631 & 66.990 & $\begin{array}{l}\mathrm{O}=\mathrm{C}(\mathrm{OCCNC}(=\mathrm{O}) \mathrm{OC}(\mathrm{C})(\mathrm{C}) \mathrm{C}) \mathrm{N}[\mathrm{C} @ @]([\mathrm{H}])(\mathrm{C} / \mathrm{C} 1=\mathrm{C} / \mathrm{C}=\mathrm{C} \\
\mathrm{C}=\mathrm{C} 1)[\mathrm{C} @](\mathrm{O})([\mathrm{H}]) \mathrm{CN}(\mathrm{CCCC})[\mathrm{S}](=\mathrm{O})(=\mathrm{O}) \mathrm{C} 2=\mathrm{CC}=\mathrm{C}(\mathrm{C} \\
=\mathrm{C} 2) \mathrm{OC}\end{array}$ \\
\hline $\mathrm{H} 27$ & - & 3.400 & 3.626 & 78.339 & 3.594 & 91.505 & $\mathrm{O}=\mathrm{C}(\mathrm{OCCN} 1 \mathrm{CCNC} 1=\mathrm{O}) \mathrm{N}[\mathrm{C} @ @]([\mathrm{H}])(\mathrm{C} / \mathrm{C} 2=\mathrm{C} / \mathrm{C}=\mathrm{CC}=\mathrm{C} 2$ \\
\hline
\end{tabular}




\begin{tabular}{|c|c|c|c|c|c|c|c|}
\hline & & & & & & & $\begin{array}{l})[\mathrm{C} @](\mathrm{O})([\mathrm{H}]) \mathrm{CN}(\mathrm{CCCCC})[\mathrm{S}](=\mathrm{O})(=\mathrm{O}) \mathrm{C} 3=\mathrm{CC}=\mathrm{C}(\mathrm{C}=\mathrm{C} 3) \mathrm{O} \\
\mathrm{C}\end{array}$ \\
\hline H39 & - & 1.376 & 0.872 & 18.838 & 0.847 & 21.555 & $\begin{array}{l}\mathrm{O}=\mathrm{C}(\mathrm{O}[\mathrm{C} @] 1([\mathrm{H}]) \mathrm{CCOC} 1=\mathrm{O}) \mathrm{N}[\mathrm{C} @ @]([\mathrm{H}])(\mathrm{C} / \mathrm{C} 2=\mathrm{C} / \mathrm{C}=\mathrm{C} \\
\mathrm{C}=\mathrm{C} 2)[\mathrm{C} @](\mathrm{O})([\mathrm{H}]) \mathrm{CN}(\mathrm{CCCCC})[\mathrm{S}](=\mathrm{O})(=\mathrm{O}) \mathrm{C} 4=\mathrm{CC}=\mathrm{C} 3 \mathrm{~N} \\
=\mathrm{C}[\mathrm{S}] \mathrm{C} 3=\mathrm{C} 4\end{array}$ \\
\hline $\mathrm{H} 43$ & - & 4.180 & 4.232 & 91.430 & 4.273 & 108.800 & $\begin{array}{l}\mathrm{O}=\mathrm{C} 1 \mathrm{O}[\mathrm{C} @ @]([\mathrm{H}])(\mathrm{CN} 1 \mathrm{C}=2 \mathrm{C}=\mathrm{CC}([\mathrm{F}])=\mathrm{C}([\mathrm{F}]) \mathrm{C}=2) \mathrm{C}(=\mathrm{O}) \\
\mathrm{N}[\mathrm{C} @ @]([\mathrm{H}])(\mathrm{C} / \mathrm{C} 3=\mathrm{C} / \mathrm{C}=\mathrm{CC}=\mathrm{C} 3)[\mathrm{C} @ @](\mathrm{O})([\mathrm{H}]) \mathrm{CN}(\mathrm{CC}( \\
\mathrm{C})(\mathrm{C})[\mathrm{H}])[\mathrm{S}](=\mathrm{O})(=\mathrm{O}) \mathrm{C} 4=\mathrm{CC}=\mathrm{C}(\mathrm{C}=\mathrm{C} 4) \mathrm{OC}\end{array}$ \\
\hline H47 & - & 4.347 & 4.753 & 102.699 & 4.714 & 120.024 & $\begin{array}{l}\mathrm{O}=\mathrm{C} 1 \mathrm{O}[\mathrm{C} @ @]([\mathrm{H}])(\mathrm{CN} 1 \mathrm{C}=2 \mathrm{C}=\mathrm{CC}=\mathrm{C}(\mathrm{C}=2) \mathrm{OC}) \mathrm{C}(=\mathrm{O}) \mathrm{N}[\mathrm{C} \\
@ @ @]([\mathrm{H}])(\mathrm{C} / \mathrm{C} 3=\mathrm{C} / \mathrm{C}=\mathrm{CC}=\mathrm{C} 3)[\mathrm{C} @ @](\mathrm{O})([\mathrm{H}]) \mathrm{CN}(\mathrm{CC}(\mathrm{C})( \\
\mathrm{C})[\mathrm{H}])[\mathrm{S}](=\mathrm{O})(=\mathrm{O}) \mathrm{C} 4=\mathrm{CC}=\mathrm{C}(\mathrm{C}=\mathrm{C} 4) \mathrm{OC}\end{array}$ \\
\hline H52 & - & 3.495 & 4.289 & 92.669 & 4.271 & 108.747 & $\begin{array}{l}\mathrm{O}=\mathrm{C} 1 \mathrm{O}[\mathrm{C} @ @]([\mathrm{H}])(\mathrm{CN} 1 \mathrm{C}=2 \mathrm{C}=\mathrm{CC}=\mathrm{C}(\mathrm{C}=2) \mathrm{C}(=\mathrm{O}) \mathrm{C}) \mathrm{C}(=\mathrm{O} \\
) \mathrm{N}[\mathrm{C} @ @]([\mathrm{H}])(\mathrm{C} / \mathrm{C} 3=\mathrm{C} / \mathrm{C}=\mathrm{CC}=\mathrm{C} 3)[\mathrm{C} @ @](\mathrm{O})([\mathrm{H}]) \mathrm{CN}(\mathrm{CC} \\
(\mathrm{C})(\mathrm{C})[\mathrm{H}])[\mathrm{S}](=\mathrm{O})(=\mathrm{O}) \mathrm{C} 4=\mathrm{C} / \mathrm{C}=\mathrm{C}(/ \mathrm{N}) \mathrm{C}=\mathrm{C} 4\end{array}$ \\
\hline H58 & - & 4.796 & 4.476 & 96.711 & 4.498 & 114.521 & $\begin{array}{l}\mathrm{O}=\mathrm{C} 1 \mathrm{O}[\mathrm{C} @ @]([\mathrm{H}])(\mathrm{CN} 1 \mathrm{C}=2 \mathrm{C}=\mathrm{CC}(=\mathrm{CC}=2) \mathrm{C}(=\mathrm{O}) \mathrm{C}) \mathrm{C}(=\mathrm{O} \\
) \mathrm{N}[\mathrm{C} @ @]([\mathrm{H}])(\mathrm{C} / \mathrm{C} 3=\mathrm{C} / \mathrm{C}=\mathrm{CC}=\mathrm{C} 3)[\mathrm{C} @ @](\mathrm{O})([\mathrm{H}]) \mathrm{CN}(\mathrm{CC} \\
(\mathrm{C})(\mathrm{C})[\mathrm{H}])[\mathrm{S}](=\mathrm{O})(=\mathrm{O}) \mathrm{C} 5=\mathrm{CC}=\mathrm{C} 4 \mathrm{OCOC} 4=\mathrm{C} 5\end{array}$ \\
\hline H64 & - & 2.000 & 2.216 & 47.878 & 2.241 & 57.063 & $\begin{array}{l}\mathrm{O}=\mathrm{C} 1 \mathrm{O}[\mathrm{C} @ @]([\mathrm{H}])(\mathrm{CN} 1 \mathrm{C}=2 \mathrm{C}=\mathrm{CC}=\mathrm{C}(\mathrm{C}=2) \mathrm{C}([\mathrm{F}])([\mathrm{F}])[\mathrm{F}]) \\
\mathrm{C}(=\mathrm{O}) \mathrm{N}[\mathrm{C} @ @]([\mathrm{H}])(\mathrm{C} / \mathrm{C} 3=\mathrm{C} / \mathrm{C}=\mathrm{CC}=\mathrm{C} 3)[\mathrm{C} @ @](\mathrm{O})([\mathrm{H}]) \mathrm{C} \\
\mathrm{N}(\mathrm{CC}(\mathrm{C})(\mathrm{C})[\mathrm{H}])[\mathrm{S}](=\mathrm{O})(=\mathrm{O}) \mathrm{C} 4=\mathrm{CC}=\mathrm{C}(\mathrm{C}=\mathrm{C} 4) \mathrm{OC}([\mathrm{F}])([\mathrm{F}]) \\
{[\mathrm{F}] \sim}\end{array}$ \\
\hline H74 & - & 0.769 & 0.671 & 14.499 & 0.700 & 17.827 & $\begin{array}{l}\mathrm{O}=\mathrm{C} 1 \mathrm{O}[\mathrm{C} @ @]([\mathrm{H}])(\mathrm{CN} 1 \mathrm{C}=2 \mathrm{C}=\mathrm{CC}=\mathrm{CC}=2) \mathrm{C}(=\mathrm{O}) \mathrm{N}[\mathrm{C} @ @ \\
]([\mathrm{H}])(\mathrm{C} / \mathrm{C} 3=\mathrm{C} / \mathrm{C}=\mathrm{CC}=\mathrm{C} 3)[\mathrm{C} @ @](\mathrm{O})([\mathrm{H}]) \mathrm{CN}(\mathrm{C} / \mathrm{C} 4=\mathrm{C} / \mathrm{C}= \\
\mathrm{C}[\mathrm{S}] 4)[\mathrm{S}](=\mathrm{O})(=\mathrm{O}) \mathrm{C} 5=\mathrm{C} C([\mathrm{~F}])=\mathrm{C}([\mathrm{F}]) \mathrm{C}=\mathrm{C} 5[\mathrm{~F}]\end{array}$ \\
\hline H82 & - & 2.133 & 2.128 & 45.977 & 2.122 & 54.024 & $\begin{array}{l}\mathrm{O}=\mathrm{C} 1 \mathrm{O}[\mathrm{C} @ @]([\mathrm{H}])(\mathrm{CN} 1 \mathrm{C}=2 \mathrm{C}=\mathrm{CC}=\mathrm{CC}=2 \mathrm{O}) \mathrm{C}(=\mathrm{O}) \mathrm{N}[\mathrm{C} @ \\
@]([\mathrm{H}])(\mathrm{C} / \mathrm{C} 3=\mathrm{C} / \mathrm{C}=\mathrm{CC}=\mathrm{C} 3)[\mathrm{C} @ @](\mathrm{O})([\mathrm{H}]) \mathrm{CN}(\mathrm{CC}(\mathrm{C})(\mathrm{C})[ \\
\mathrm{H}])[\mathrm{S}](=\mathrm{O})(=\mathrm{O}) \mathrm{C} 4=\mathrm{C} / \mathrm{C}=\mathrm{C}(/ \mathrm{N}) \mathrm{C}=\mathrm{C} 4\end{array}$ \\
\hline H88 & - & 4.013 & 4.221 & 91.209 & 4.226 & 107.596 & $\begin{array}{l}\mathrm{O}=\mathrm{C} 1 \mathrm{O}[\mathrm{C} @ @]([\mathrm{H}])(\mathrm{CN} 1 \mathrm{C}=2 \mathrm{C}=\mathrm{CC}=\mathrm{CC}=2 \mathrm{C}([\mathrm{F}])([\mathrm{F}])[\mathrm{F}]) \mathrm{C} \\
(=\mathrm{O}) \mathrm{N}[\mathrm{C} @ @]([\mathrm{H}])(\mathrm{C} / \mathrm{C} 3=\mathrm{C} / \mathrm{C}=\mathrm{CC}=\mathrm{C} 3)[\mathrm{C} @ @](\mathrm{O})([\mathrm{H}]) \mathrm{CN} \\
(\mathrm{CC}(\mathrm{C})(\mathrm{C})[\mathrm{H}])[\mathrm{S}](=\mathrm{O})(=\mathrm{O}) \mathrm{C} 5=\mathrm{CC}=\mathrm{C} 4 \mathrm{~N}=\mathrm{C}[\mathrm{S}] \mathrm{C} 4=\mathrm{C} 5\end{array}$ \\
\hline H96 & - & 3.893 & 4.106 & 88.714 & 3.998 & 101.798 & $\begin{array}{l}\mathrm{O}=\mathrm{C} 1 \mathrm{O}[\mathrm{C} @ @]([\mathrm{H}])(\mathrm{CN} 1 \mathrm{C}=2 \mathrm{C}=\mathrm{CC}([\mathrm{F}])=\mathrm{CC}=2) \mathrm{C}(=\mathrm{O}) \mathrm{N}[\mathrm{C} \\
@ @]([\mathrm{H}])(\mathrm{C} / \mathrm{C} 3=\mathrm{C} / \mathrm{C}=\mathrm{CC}=\mathrm{C} 3)[\mathrm{C} @ @](\mathrm{O})([\mathrm{H}]) \mathrm{CN}(\mathrm{CC}(\mathrm{C})( \\
\mathrm{C})[\mathrm{H}])[\mathrm{S}](=\mathrm{O})(=\mathrm{O}) \mathrm{C} 4=\mathrm{CC}=\mathrm{C}(\mathrm{C}=\mathrm{C} 4) \mathrm{OC}\end{array}$ \\
\hline H108 & - & 3.648 & 3.502 & 75.663 & 3.507 & 89.293 & $\begin{array}{l}\mathrm{O}=\mathrm{C} 1 \mathrm{O}[\mathrm{C} @ @]([\mathrm{H}])(\mathrm{CN} 1 \mathrm{C}=2 \mathrm{C}=\mathrm{CC}=\mathrm{C}(\mathrm{C}=2) \mathrm{OC}([\mathrm{F}])([\mathrm{F}])[\mathrm{F} \\
]) \mathrm{C}(=\mathrm{O}) \mathrm{N}[\mathrm{C} @ @]([\mathrm{H}])(\mathrm{C} / \mathrm{C} 3=\mathrm{C} / \mathrm{C}=\mathrm{CC}=\mathrm{C} 3)[\mathrm{C} @ @](\mathrm{O})([\mathrm{H}]) \\
\mathrm{CN}(\mathrm{CC}(\mathrm{C})(\mathrm{C})[\mathrm{H}])[\mathrm{S}](=\mathrm{O})(=\mathrm{O}) \mathrm{C} 4=\mathrm{C} / \mathrm{C}=\mathrm{C}(/ \mathrm{N}) \mathrm{C}=\mathrm{C} 4\end{array}$ \\
\hline H115 & - & 4.310 & 4.601 & 99.406 & 4.616 & 117.520 & $\begin{array}{l}\mathrm{O}=\mathrm{C} 1 \mathrm{O}[\mathrm{C} @ @]([\mathrm{H}])(\mathrm{CN} 1 \mathrm{C}=2 \mathrm{C}=\mathrm{CC}=\mathrm{C}(\mathrm{C}=2)[\mathrm{S}](\mathrm{C})(=\mathrm{O})=\mathrm{O}) \\
\mathrm{C}(=\mathrm{O}) \mathrm{N}[\mathrm{C} @ @]([\mathrm{H}])(\mathrm{C} / \mathrm{C} 3=\mathrm{C} / \mathrm{C}=\mathrm{CC}=\mathrm{C} 3)[\mathrm{C} @ @](\mathrm{O})([\mathrm{H}]) \mathrm{C} \\
\mathrm{N}(\mathrm{CC}(\mathrm{C})(\mathrm{C})[\mathrm{H}])[\mathrm{S}](=\mathrm{O})(=\mathrm{O}) \mathrm{C} 4=\mathrm{CC}=\mathrm{C}(\mathrm{C}=\mathrm{C} 4) \mathrm{OC}\end{array}$ \\
\hline H120 & - & 4.824 & 4.590 & 99.183 & 4.599 & 117.087 & $\begin{array}{l}\mathrm{O}=\mathrm{C} 1 \mathrm{O}[\mathrm{C} @ @]([\mathrm{H}])(\mathrm{CN} 1 \mathrm{C}=2 \mathrm{C}=\mathrm{CC}=\mathrm{C}(\mathrm{C}=2)[\mathrm{N}+](=\mathrm{O})[\mathrm{O}- \\
]) \mathrm{C}(=\mathrm{O}) \mathrm{N}[\mathrm{C} @ @]([\mathrm{H}])(\mathrm{C} / \mathrm{C} 3=\mathrm{C} / \mathrm{C}=\mathrm{CC}=\mathrm{C} 3)[\mathrm{C} @ @](\mathrm{O})([\mathrm{H}]) \\
\mathrm{CN}(\mathrm{CC}(\mathrm{C})(\mathrm{C})[\mathrm{H}])[\mathrm{S}](=\mathrm{O})(=\mathrm{O}) \mathrm{C} 5=\mathrm{CC}=\mathrm{C} 4 \mathrm{~N}=\mathrm{C}[\mathrm{S}] \mathrm{C} 4=\mathrm{C} 5\end{array}$ \\
\hline H128 & - & 3.539 & 3.897 & 84.210 & 3.958 & 100.764 & $\begin{array}{l}\mathrm{O}=\mathrm{C} 1 \mathrm{O}[\mathrm{C} @ @]([\mathrm{H}])(\mathrm{CN} 1 \mathrm{C}=2 \mathrm{C}=\mathrm{CC}=\mathrm{C}(\mathrm{C}=2) \mathrm{NC}(=\mathrm{O}) \mathrm{OC}) \mathrm{C}( \\
=\mathrm{O}) \mathrm{N}[\mathrm{C} @ @]([\mathrm{H}])(\mathrm{C} / \mathrm{C} 3=\mathrm{C} / \mathrm{C}=\mathrm{CC}=\mathrm{C} 3)[\mathrm{C} @ @](\mathrm{O})([\mathrm{H}]) \mathrm{CN}( \\
\mathrm{CC}(\mathrm{C})(\mathrm{C})[\mathrm{H}])[\mathrm{S}](=\mathrm{O})(=\mathrm{O}) \mathrm{C} 5=\mathrm{CC}=\mathrm{C} 4 \mathrm{OCOC} 4=\mathrm{C} 5\end{array}$ \\
\hline $\mathrm{H} 3$ & $\#$ & 1.923 & 2.108 & 45.543 & 2.300 & 58.563 & $\begin{array}{l}\mathrm{O}=\mathrm{C}(\mathrm{OCCNC}(=\mathrm{O}) \mathrm{C}([\mathrm{F}])([\mathrm{F}])[\mathrm{F}]) \mathrm{N}[\mathrm{C} @ @]([\mathrm{H}])(\mathrm{C} / \mathrm{C} 1=\mathrm{C} / \mathrm{C} \\
=\mathrm{CC}=\mathrm{C} 1)[\mathrm{C} @](\mathrm{O})([\mathrm{H}]) \mathrm{CN}(\mathrm{C}[\mathrm{C} @ @](\mathrm{C})([\mathrm{H}]) \mathrm{CC})[\mathrm{S}](=\mathrm{O})(= \\
\mathrm{O}) \mathrm{C} 2=\mathrm{CC}=\mathrm{C}(\mathrm{C}=\mathrm{C} 2) \mathrm{OC}\end{array}$ \\
\hline $\mathrm{H} 10$ & $\#$ & 1.455 & 1.102 & 23.814 & 1.062 & 27.046 & $\mathrm{O}=\mathrm{C}(\mathrm{O}[\mathrm{C} @] 1([\mathrm{H}]) \mathrm{CCOC} 1=\mathrm{O}) \mathrm{N}[\mathrm{C} @ @]([\mathrm{H}])(\mathrm{C} / \mathrm{C} 2=\mathrm{C} / \mathrm{C}=\mathrm{C}$ \\
\hline
\end{tabular}




\begin{tabular}{|c|c|c|c|c|c|c|c|}
\hline & & & & & & & $\begin{array}{l}\mathrm{C}=\mathrm{C} 2)[\mathrm{C} @](\mathrm{O})([\mathrm{H}]) \mathrm{CN}(\mathrm{C}[\mathrm{C} @ @](\mathrm{C})([\mathrm{H}]) \mathrm{CC})[\mathrm{S}](=\mathrm{O})(=\mathrm{O}) \\
\mathrm{C} 3=\mathrm{CC}=\mathrm{C}(\mathrm{C}=\mathrm{C} 3) \mathrm{OC}\end{array}$ \\
\hline H13 & $\#$ & 2.040 & 2.040 & 44.069 & 2.262 & 57.592 & $\begin{array}{l}\mathrm{O}=\mathrm{C}(\mathrm{OCCNC}(=\mathrm{O}) \mathrm{C}([\mathrm{F}])([\mathrm{F}])[\mathrm{F}]) \mathrm{N}[\mathrm{C} @ @]([\mathrm{H}])(\mathrm{C} / \mathrm{C} 1=\mathrm{C} / \mathrm{C} \\
=\mathrm{CC}=\mathrm{C} 1)[\mathrm{C} @](\mathrm{O})([\mathrm{H}]) \mathrm{CN}(\mathrm{C}[\mathrm{C} @ @](\mathrm{C})([\mathrm{H}]) \mathrm{CC})[\mathrm{S}](=\mathrm{O})(= \\
\mathrm{O}) \mathrm{C} 3=\mathrm{CC}=\mathrm{C} 2 \mathrm{~N}=\mathrm{C}[\mathrm{S}] \mathrm{C} 2=\mathrm{C} 3\end{array}$ \\
\hline H17 & $\#$ & 2.856 & 3.926 & 84.822 & 3.970 & 101.086 & $\begin{array}{l}\mathrm{O}=\mathrm{C}(\mathrm{OC}[\mathrm{C} @](\mathrm{C})([\mathrm{H}]) \mathrm{C}(=\mathrm{O}) \mathrm{OC}) \mathrm{N}[\mathrm{C} @ @]([\mathrm{H}])(\mathrm{C} / \mathrm{C} 1=\mathrm{C} / \mathrm{C} \\
=\mathrm{CC}=\mathrm{C} 1)[\mathrm{C} @](\mathrm{O})([\mathrm{H}]) \mathrm{CN}(\mathrm{C}[\mathrm{C} @ @](\mathrm{C})([\mathrm{H}]) \mathrm{CC})[\mathrm{S}](=\mathrm{O})(= \\
\mathrm{O}) \mathrm{C} 3=\mathrm{CC}=\mathrm{C} 2 \mathrm{~N}=\mathrm{C}[\mathrm{S}] \mathrm{C} 2=\mathrm{C} 3\end{array}$ \\
\hline H29 & $\#$ & 1.181 & 0.444 & 9.589 & 0.384 & 9.770 & $\begin{array}{l}\mathrm{O}=\mathrm{C}(\mathrm{O}[\mathrm{C} @] 1([\mathrm{H}]) \mathrm{CCOC} 1=\mathrm{O}) \mathrm{N}[\mathrm{C} @ @]([\mathrm{H}])(\mathrm{C} / \mathrm{C} 2=\mathrm{C} / \mathrm{C}=\mathrm{C} \\
\mathrm{C}=\mathrm{C} 2)[\mathrm{C} @](\mathrm{O})([\mathrm{H}]) \mathrm{CN}(\mathrm{CCCCC})[\mathrm{S}](=\mathrm{O})(=\mathrm{O}) \mathrm{C} 3=\mathrm{CC}=\mathrm{C}(\mathrm{C} \\
=\mathrm{C} 3) \mathrm{OC}\end{array}$ \\
\hline H34 & $\#$ & 2.472 & 2.225 & 48.071 & 2.437 & 62.046 & $\begin{array}{l}\mathrm{O}=\mathrm{C}(\mathrm{O}[\mathrm{C} @ @](\mathrm{C})([\mathrm{H}]) \mathrm{CCC}(\mathrm{C})=\mathrm{O}) \mathrm{N}[\mathrm{C} @ @]([\mathrm{H}])(\mathrm{C} / \mathrm{C} 1=\mathrm{C} / \\
\mathrm{C}=\mathrm{CC}=\mathrm{C} 1)[\mathrm{C} @](\mathrm{O})([\mathrm{H}]) \mathrm{CN}(\mathrm{CCCCC})[\mathrm{S}](=\mathrm{O})(=\mathrm{O}) \mathrm{C} 3=\mathrm{CC}= \\
\mathrm{C} 2 \mathrm{~N}=\mathrm{C}[\mathrm{S}] \mathrm{C} 2=\mathrm{C} 3\end{array}$ \\
\hline H44 & $\#$ & 5.222 & 3.895 & 84.159 & 3.910 & 99.544 & $\begin{array}{l}\mathrm{O}=\mathrm{C} 1 \mathrm{O}[\mathrm{C} @ @]([\mathrm{H}])(\mathrm{CN} 1 \mathrm{C}=2 \mathrm{C}=\mathrm{CC}=\mathrm{C}(\mathrm{C}=2) \mathrm{C}([\mathrm{F}])([\mathrm{F}])[\mathrm{F}]) \\
\mathrm{C}(=\mathrm{O}) \mathrm{N}[\mathrm{C} @ @]([\mathrm{H}])(\mathrm{C} / \mathrm{C} 3=\mathrm{C} / \mathrm{C}=\mathrm{CC}=\mathrm{C} 3)[\mathrm{C} @ @](\mathrm{O})([\mathrm{H}]) \mathrm{C} \\
\mathrm{N}(\mathrm{CC}(\mathrm{C})(\mathrm{C})[\mathrm{H}])[\mathrm{S}](=\mathrm{O})(=\mathrm{O}) \mathrm{C} 4=\mathrm{CC}=\mathrm{C}(\mathrm{C}=\mathrm{C} 4) \mathrm{OC}\end{array}$ \\
\hline H50 & $\#$ & 3.638 & 3.791 & 81.922 & 3.792 & 96.537 & $\begin{array}{l}\mathrm{O}=\mathrm{C} 1 \mathrm{O}[\mathrm{C} @ @]([\mathrm{H}])(\mathrm{CN} 1 \mathrm{C}=2 \mathrm{C}=\mathrm{CC}([\mathrm{F}])=\mathrm{C}([\mathrm{F}]) \mathrm{C}=2) \mathrm{C}(=\mathrm{O}) \\
\mathrm{N}[\mathrm{C} @ @]([\mathrm{H}])(\mathrm{C} / \mathrm{C} 3=\mathrm{C} / \mathrm{C}=\mathrm{CC}=\mathrm{C} 3)[\mathrm{C} @ @](\mathrm{O})([\mathrm{H}]) \mathrm{CN}(\mathrm{CC}( \\
\mathrm{C})(\mathrm{C})[\mathrm{H}])[\mathrm{S}](=\mathrm{O})(=\mathrm{O}) \mathrm{C} 4=\mathrm{C} / \mathrm{C}=\mathrm{C}(/ \mathrm{N}) \mathrm{C}=\mathrm{C} 4\end{array}$ \\
\hline H55 & \# & 4.071 & 4.360 & 94.195 & 4.391 & 111.799 & $\begin{array}{l}\mathrm{O}=\mathrm{C} 1 \mathrm{O}[\mathrm{C} @ @]([\mathrm{H}])(\mathrm{CN} 1 \mathrm{C}=2 \mathrm{C}=\mathrm{CC}([\mathrm{F}])=\mathrm{C}([\mathrm{F}]) \mathrm{C}=2) \mathrm{C}(=\mathrm{O}) \\
\mathrm{N}[\mathrm{C} @ @]([\mathrm{H}])(\mathrm{C} / \mathrm{C} 3=\mathrm{C} / \mathrm{C}=\mathrm{CC}=\mathrm{C} 3)[\mathrm{C} @ @](\mathrm{O})([\mathrm{H}]) \mathrm{CN}(\mathrm{CC}( \\
\mathrm{C})(\mathrm{C})[\mathrm{H}])[\mathrm{S}](=\mathrm{O})(=\mathrm{O}) \mathrm{C} 5=\mathrm{CC}=\mathrm{C} 4 \mathrm{OCOC} 4=\mathrm{C} 5\end{array}$ \\
\hline H61 & $\#$ & 4.143 & 3.443 & 74.389 & 3.438 & 87.523 & $\begin{array}{l}\mathrm{O}=\mathrm{C} 1 \mathrm{O}[\mathrm{C} @ @]([\mathrm{H}])(\mathrm{CN} 1 \mathrm{C}=2 \mathrm{C}=\mathrm{CC}=\mathrm{C}(\mathrm{C}=2) \mathrm{C}([\mathrm{F}])([\mathrm{F}])[\mathrm{F}]) \\
\mathrm{C}(=\mathrm{O}) \mathrm{N}[\mathrm{C} @ @]([\mathrm{H}])(\mathrm{C} / \mathrm{C} 3=\mathrm{C} / \mathrm{C}=\mathrm{CC}=\mathrm{C} 3)[\mathrm{C} @ @](\mathrm{O})([\mathrm{H}]) \mathrm{C} \\
\mathrm{N}(\mathrm{CC}(\mathrm{C})(\mathrm{C})[\mathrm{H}])[\mathrm{S}](=\mathrm{O})(=\mathrm{O}) \mathrm{C} 4=\mathrm{C} / \mathrm{C}=\mathrm{C}(/ \mathrm{OC}) \mathrm{C}(\mathrm{C})=\mathrm{C} 4\end{array}$ \\
\hline H68 & $\#$ & 3.590 & 2.950 & 63.738 & 2.889 & 73.563 & $\begin{array}{l}\mathrm{O}=\mathrm{C} 1 \mathrm{O}[\mathrm{C} @ @]([\mathrm{H}])(\mathrm{CN} 1 \mathrm{C}=2 \mathrm{C}=\mathrm{C} / \mathrm{C}=\mathrm{C}(/[\mathrm{F}]) \mathrm{C}=2) \mathrm{C}(=\mathrm{O}) \mathrm{N}[ \\
\mathrm{C} @ @]([\mathrm{H}])(\mathrm{C} / \mathrm{C} 3=\mathrm{C} / \mathrm{C}=\mathrm{CC}=\mathrm{C} 3)[\mathrm{C} @ @](\mathrm{O})([\mathrm{H}]) \mathrm{CN}(\mathrm{C}[\mathrm{C} @ \\
@] 4([\mathrm{H}]) \mathrm{CC} 4)[\mathrm{S}](=\mathrm{O})(=\mathrm{O}) \mathrm{C} 5=\mathrm{CC}=\mathrm{C}(\mathrm{C}=\mathrm{C} 5) \mathrm{OC}\end{array}$ \\
\hline H75 & $\#$ & 0.795 & 0.253 & 5.461 & 0.474 & 12.067 & $\begin{array}{l}\mathrm{O}=\mathrm{C} 1 \mathrm{O}[\mathrm{C} @ @]([\mathrm{H}])(\mathrm{CN} 1 \mathrm{C}=2 \mathrm{C}=\mathrm{C} / \mathrm{C}=\mathrm{C}(/[\mathrm{F}]) \mathrm{C}=2) \mathrm{C}(=\mathrm{O}) \mathrm{N}[ \\
\mathrm{C} @ @]([\mathrm{H}])(\mathrm{C} / \mathrm{C} 3=\mathrm{C} / \mathrm{C}=\mathrm{CC}=\mathrm{C} 3)[\mathrm{C} @ @](\mathrm{O})([\mathrm{H}]) \mathrm{CN}(\mathrm{C} / \mathrm{C} 4= \\
\mathrm{C} / \mathrm{C}=\mathrm{C}[\mathrm{S}] 4)[\mathrm{S}](=\mathrm{O})(=\mathrm{O}) \mathrm{C} 5=\mathrm{CC}([\mathrm{F}])=\mathrm{C}([\mathrm{F}]) \mathrm{C}=\mathrm{C} 5[\mathrm{~F}]\end{array}$ \\
\hline H86 & $\#$ & 4.066 & 3.850 & 83.194 & 3.871 & 98.557 & $\begin{array}{l}\mathrm{O}=\mathrm{C} 1 \mathrm{O}[\mathrm{C} @ @]([\mathrm{H}])(\mathrm{CN} 1 \mathrm{C}=2 \mathrm{C}=\mathrm{CC}=\mathrm{CC}=2 \mathrm{C}([\mathrm{F}])([\mathrm{F}])[\mathrm{F}]) \mathrm{C} \\
(=\mathrm{O}) \mathrm{N}[\mathrm{C} @ @]([\mathrm{H}])(\mathrm{C} / \mathrm{C} 3=\mathrm{C} / \mathrm{C}=\mathrm{CC}=\mathrm{C} 3)[\mathrm{C} @ @](\mathrm{O})([\mathrm{H}]) \mathrm{CN} \\
(\mathrm{CC}(\mathrm{C})(\mathrm{C})[\mathrm{H}])[\mathrm{S}](=\mathrm{O})(=\mathrm{O}) \mathrm{C} 4=\mathrm{CC}=\mathrm{C}(\mathrm{C}=\mathrm{C} 4) \mathrm{OC}\end{array}$ \\
\hline $\mathrm{H} 90$ & $\#$ & 3.415 & 3.215 & 69.459 & 3.194 & 81.308 & $\begin{array}{l}\mathrm{O}=\mathrm{C} 1 \mathrm{O}[\mathrm{C} @ @]([\mathrm{H}])(\mathrm{CN} 1 \mathrm{C}=2 \mathrm{C}=\mathrm{CC}([\mathrm{F}])=\mathrm{CC}=2[\mathrm{~F}]) \mathrm{C}(=\mathrm{O}) \mathrm{N} \\
{[\mathrm{C} @ @]([\mathrm{H}])(\mathrm{C} / \mathrm{C} 3=\mathrm{C} / \mathrm{C}=\mathrm{CC}=\mathrm{C} 3)[\mathrm{C} @ @](\mathrm{O})([\mathrm{H}]) \mathrm{CN}(\mathrm{CC}(\mathrm{C}} \\
)(\mathrm{C})[\mathrm{H}])[\mathrm{S}](=\mathrm{O})(=\mathrm{O}) \mathrm{C} 4=\mathrm{C} / \mathrm{C}=\mathrm{C}(/ \mathrm{N}) \mathrm{C}=\mathrm{C} 4\end{array}$ \\
\hline H94 & $\#$ & 3.674 & 3.018 & 65.220 & 3.017 & 76.811 & $\begin{array}{l}\mathrm{O}=\mathrm{C} 1 \mathrm{O}[\mathrm{C} @ @]([\mathrm{H}])(\mathrm{CN} 1 \mathrm{C}=2 \mathrm{C}=\mathrm{CC}([\mathrm{F}])=\mathrm{CC}=2[\mathrm{~F}]) \mathrm{C}(=\mathrm{O}) \mathrm{N} \\
{[\mathrm{C} @ @]([\mathrm{H}])(\mathrm{C} / \mathrm{C} 3=\mathrm{C} / \mathrm{C}=\mathrm{CC}=\mathrm{C} 3)[\mathrm{C} @ @](\mathrm{O})([\mathrm{H}]) \mathrm{CN}(\mathrm{CC}(\mathrm{C}} \\
)(\mathrm{C})[\mathrm{H}])[\mathrm{S}](=\mathrm{O})(=\mathrm{O}) \mathrm{C} 4=\mathrm{CC}=\mathrm{C}(\mathrm{C}=\mathrm{C} 4) \mathrm{CO}\end{array}$ \\
\hline H99 & $\#$ & 3.684 & 3.470 & 74.965 & 3.340 & 85.038 & $\begin{array}{l}\mathrm{O}=\mathrm{C} 1 \mathrm{O}[\mathrm{C} @ @]([\mathrm{H}])(\mathrm{CN} 1 \mathrm{C}=2 \mathrm{C}=\mathrm{CC}([\mathrm{F}])=\mathrm{CC}=2) \mathrm{C}(=\mathrm{O}) \mathrm{N}[\mathrm{C} \\
@ @]([\mathrm{H}])(\mathrm{C} / \mathrm{C} 3=\mathrm{C} / \mathrm{C}=\mathrm{CC}=\mathrm{C} 3)[\mathrm{C} @ @](\mathrm{O})([\mathrm{H}]) \mathrm{CN}(\mathrm{CC}(\mathrm{C})( \\
\mathrm{C})[\mathrm{H}])[\mathrm{S}](=\mathrm{O})(=\mathrm{O}) \mathrm{C} 4=\mathrm{CC}=\mathrm{C}(\mathrm{C}=\mathrm{C} 4) \mathrm{CO}\end{array}$ \\
\hline H103 & $\#$ & 3.496 & 3.445 & 74.442 & 3.418 & 87.016 & $\begin{array}{l}\mathrm{O}=\mathrm{C} 1 \mathrm{O}[\mathrm{C} @ @]([\mathrm{H}])(\mathrm{CN} 1 \mathrm{C}=2 \mathrm{C}=\mathrm{C} / \mathrm{C}=\mathrm{C}(/[\mathrm{F}]) \mathrm{C}=2) \mathrm{C}(=\mathrm{O}) \mathrm{N}[ \\
\mathrm{C} @ @]([\mathrm{H}])(\mathrm{C} / \mathrm{C} 3=\mathrm{C} / \mathrm{C}=\mathrm{CC}=\mathrm{C} 3)[\mathrm{C} @ @](\mathrm{O})([\mathrm{H}]) \mathrm{CN}(\mathrm{CC}(\mathrm{C}) \\
(\mathrm{C})[\mathrm{H}])[\mathrm{S}](=\mathrm{O})(=\mathrm{O}) \mathrm{C} 4=\mathrm{CC}=\mathrm{C}(\mathrm{C}=\mathrm{C} 4) \mathrm{CO}\end{array}$ \\
\hline H112 & $\#$ & 3.544 & 3.306 & 71.424 & 3.331 & 84.796 & $\begin{array}{l}\mathrm{O}=\mathrm{C} 1 \mathrm{O}[\mathrm{C} @ @]([\mathrm{H}])(\mathrm{CN} 1 \mathrm{C}=2 \mathrm{C}=\mathrm{CC}=\mathrm{C}(\mathrm{C}=2) \mathrm{OC}([\mathrm{F}])([\mathrm{F}])[\mathrm{F} \\
]) \mathrm{C}(=\mathrm{O}) \mathrm{N}[\mathrm{C} @ @]([\mathrm{H}])(\mathrm{C} / \mathrm{C} 3=\mathrm{C} / \mathrm{C}=\mathrm{CC}=\mathrm{C} 3)[\mathrm{C} @ @](\mathrm{O})([\mathrm{H}])\end{array}$ \\
\hline
\end{tabular}




\begin{tabular}{|c|c|c|c|c|c|c|c|}
\hline & & & & & & & $\mathrm{CN}(\mathrm{CC}(\mathrm{C})(\mathrm{C})[\mathrm{H}])[\mathrm{S}](=\mathrm{O})(=\mathrm{O}) \mathrm{C} 4=\mathrm{CC}=\mathrm{C}(\mathrm{C}=\mathrm{C} 4) \mathrm{CO}$ \\
\hline H122 & \# & 3.947 & 4.800 & 103.702 & 4.930 & 125.516 & $\begin{array}{l}\mathrm{O}=\mathrm{C} 1 \mathrm{O}[\mathrm{C} @ @]([\mathrm{H}])(\mathrm{CN} 1 \mathrm{C}=2 \mathrm{C}=\mathrm{C} / \mathrm{C}=\mathrm{C}(/ \mathrm{N}) \mathrm{C}=2) \mathrm{C}(=\mathrm{O}) \mathrm{N}[\mathrm{C} \\
@ @]([\mathrm{H}])(\mathrm{C} / \mathrm{C} 3=\mathrm{C} / \mathrm{C}=\mathrm{CC}=\mathrm{C} 3)[\mathrm{C} @ @](\mathrm{O})([\mathrm{H}]) \mathrm{CN}(\mathrm{CC}(\mathrm{C})( \\
\mathrm{C})[\mathrm{H}])[\mathrm{S}](=\mathrm{O})(=\mathrm{O}) \mathrm{C} 5=\mathrm{CC}=\mathrm{C} 4 \mathrm{OCOC} 4=\mathrm{C} 5\end{array}$ \\
\hline H5 & $*$ & 3.371 & 2.951 & 63.769 & 3.154 & 80.293 & $\begin{array}{l}\mathrm{O}=\mathrm{C}(\mathrm{O}[\mathrm{C} @ @](\mathrm{C})([\mathrm{H}]) \mathrm{CCC}(\mathrm{C})=\mathrm{O}) \mathrm{N}[\mathrm{C} @ @]([\mathrm{H}])(\mathrm{C} / \mathrm{C} 1=\mathrm{C} / \\
\mathrm{C}=\mathrm{CC}=\mathrm{C} 1)[\mathrm{C} @](\mathrm{O})([\mathrm{H}]) \mathrm{CN}(\mathrm{C}[\mathrm{C} @ @](\mathrm{C})([\mathrm{H}]) \mathrm{CC})[\mathrm{S}](=\mathrm{O})( \\
=\mathrm{O}) \mathrm{C} 2=\mathrm{CC}=\mathrm{C}(\mathrm{C}=\mathrm{C} 2) \mathrm{OC}\end{array}$ \\
\hline H15 & $*$ & 3.519 & 2.883 & 62.295 & 3.116 & 79.322 & $\begin{array}{l}\mathrm{O}=\mathrm{C}(\mathrm{O}[\mathrm{C} @ @](\mathrm{C})([\mathrm{H}]) \mathrm{CCC}(\mathrm{C})=\mathrm{O}) \mathrm{N}[\mathrm{C} @ @]([\mathrm{H}])(\mathrm{C} / \mathrm{C} 1=\mathrm{C} / \\
\mathrm{C}=\mathrm{CC}=\mathrm{C} 1)[\mathrm{C} @](\mathrm{O})([\mathrm{H}]) \mathrm{CN}(\mathrm{C}[\mathrm{C} @ @](\mathrm{C})([\mathrm{H}]) \mathrm{CC})[\mathrm{S}](=\mathrm{O})( \\
=\mathrm{O}) \mathrm{C} 3=\mathrm{CC}=\mathrm{C} 2 \mathrm{~N}=\mathrm{C}[\mathrm{S}] \mathrm{C} 2=\mathrm{C} 3\end{array}$ \\
\hline $\mathrm{H} 23$ & $*$ & 1.503 & 1.449 & 31.319 & 1.622 & 41.287 & $\begin{array}{l}\mathrm{O}=\mathrm{C}(\mathrm{OCCNC}(=\mathrm{O}) \mathrm{C}([\mathrm{F}])([\mathrm{F}])[\mathrm{F}]) \mathrm{N}[\mathrm{C} @ @]([\mathrm{H}])(\mathrm{C} / \mathrm{C} 1=\mathrm{C} / \mathrm{C} \\
=\mathrm{CC}=\mathrm{C} 1)[\mathrm{C} @](\mathrm{O})([\mathrm{H}]) \mathrm{CN}(\mathrm{CCCCC})[\mathrm{S}](=\mathrm{O})(=\mathrm{O}) \mathrm{C} 2=\mathrm{CC}=\mathrm{C} \\
(\mathrm{C}=\mathrm{C} 2) \mathrm{OC}\end{array}$ \\
\hline $\mathrm{H} 32$ & $*$ & 1.706 & 1.381 & 29.845 & 1.584 & 40.316 & $\begin{array}{l}\mathrm{O}=\mathrm{C}(\mathrm{OCCNC}(=\mathrm{O}) \mathrm{C}([\mathrm{F}])([\mathrm{F}])[\mathrm{F}]) \mathrm{N}[\mathrm{C} @ @]([\mathrm{H}])(\mathrm{C} / \mathrm{C} 1=\mathrm{C} / \mathrm{C} \\
=\mathrm{CC}=\mathrm{C} 1)[\mathrm{C} @](\mathrm{O})([\mathrm{H}]) \mathrm{CN}(\mathrm{CCCCC})[\mathrm{S}](=\mathrm{O})(=\mathrm{O}) \mathrm{C} 3=\mathrm{CC}=\mathrm{C} \\
2 \mathrm{~N}=\mathrm{C}[\mathrm{S}] \mathrm{C} 2=\mathrm{C} 3\end{array}$ \\
\hline $\mathrm{H} 41$ & $*$ & 4.000 & 4.002 & 86.470 & 3.909 & 99.530 & $\begin{array}{l}\mathrm{O}=\mathrm{C} 1 \mathrm{O}[\mathrm{C} @ @]([\mathrm{H}])(\mathrm{CN} 1 \mathrm{C}=2 \mathrm{C}=\mathrm{CC}=\mathrm{CC}=2) \mathrm{C}(=\mathrm{O}) \mathrm{N}[\mathrm{C} @ @ \\
]([\mathrm{H}])(\mathrm{C} / \mathrm{C} 3=\mathrm{C} / \mathrm{C}=\mathrm{CC}=\mathrm{C} 3)[\mathrm{C} @ @](\mathrm{O})([\mathrm{H}]) \mathrm{CN}(\mathrm{CC}(\mathrm{C})(\mathrm{C})[\mathrm{H}] \\
)[\mathrm{S}](=\mathrm{O})(=\mathrm{O}) \mathrm{C} 4=\mathrm{CC}=\mathrm{C}(\mathrm{C}=\mathrm{C} 4) \mathrm{OC}\end{array}$ \\
\hline $\mathrm{H} 48$ & $*$ & 3.276 & 3.562 & 76.962 & 3.428 & 87.266 & $\begin{array}{l}\mathrm{O}=\mathrm{C} 1 \mathrm{O}[\mathrm{C} @ @]([\mathrm{H}])(\mathrm{CN} 1 \mathrm{C}=2 \mathrm{C}=\mathrm{CC}=\mathrm{CC}=2) \mathrm{C}(=\mathrm{O}) \mathrm{N}[\mathrm{C} @ @ \\
]([\mathrm{H}])(\mathrm{C} / \mathrm{C} 3=\mathrm{C} / \mathrm{C}=\mathrm{CC}=\mathrm{C} 3)[\mathrm{C} @ @](\mathrm{O})([\mathrm{H}]) \mathrm{CN}(\mathrm{CC}(\mathrm{C})(\mathrm{C})[\mathrm{H}] \\
)[\mathrm{S}](=\mathrm{O})(=\mathrm{O}) \mathrm{C} 4=\mathrm{C} / \mathrm{C}=\mathrm{C}(/ \mathrm{N}) \mathrm{C}=\mathrm{C} 4\end{array}$ \\
\hline H54 & $*$ & 3.971 & 4.210 & 90.954 & 4.194 & 106.775 & $\begin{array}{l}\mathrm{O}=\mathrm{C} 1 \mathrm{O}[\mathrm{C} @ @]([\mathrm{H}])(\mathrm{CN} 1 \mathrm{C}=2 \mathrm{C}=\mathrm{C} / \mathrm{C}=\mathrm{C}(/[\mathrm{F}]) \mathrm{C}=2) \mathrm{C}(=\mathrm{O}) \mathrm{N}[ \\
\mathrm{C} @ @]([\mathrm{H}])(\mathrm{C} / \mathrm{C} 3=\mathrm{C} / \mathrm{C}=\mathrm{CC}=\mathrm{C} 3)[\mathrm{C} @ @](\mathrm{O})([\mathrm{H}]) \mathrm{CN}(\mathrm{CC}(\mathrm{C}) \\
(\mathrm{C})[\mathrm{H}])[\mathrm{S}](=\mathrm{O})(=\mathrm{O}) \mathrm{C} 5=\mathrm{CC}=\mathrm{C} 4 \mathrm{OCOC} 4=\mathrm{C} 5\end{array}$ \\
\hline H66 & $*$ & 2.420 & 2.821 & 60.947 & 2.634 & 67.056 & $\begin{array}{l}\mathrm{O}=\mathrm{C} 1 \mathrm{O}[\mathrm{C} @ @]([\mathrm{H}])(\mathrm{CN} 1 \mathrm{C}=2 \mathrm{C}=\mathrm{CC}=\mathrm{CC}=2) \mathrm{C}(=\mathrm{O}) \mathrm{N}[\mathrm{C} @ @ \\
]([\mathrm{H}])(\mathrm{C} / \mathrm{C} 3=\mathrm{C} / \mathrm{C}=\mathrm{CC}=\mathrm{C} 3)[\mathrm{C} @ @](\mathrm{O})([\mathrm{H}]) \mathrm{CN}(\mathrm{CC}(\mathrm{C})(\mathrm{C})[\mathrm{H}] \\
)[\mathrm{S}](=\mathrm{O})(=\mathrm{O}) \mathrm{C} 4=\mathrm{CC}=\mathrm{C}(\mathrm{C}=\mathrm{C} 4) \mathrm{OC}([\mathrm{F}])([\mathrm{F}])[\mathrm{F}]\end{array}$ \\
\hline H72 & $*$ & 0.724 & 1.030 & 22.253 & 0.994 & 25.298 & $\begin{array}{l}\mathrm{O}=\mathrm{C} 1 \mathrm{O}[\mathrm{C} @ @]([\mathrm{H}])(\mathrm{CN} 1 \mathrm{C}=2 \mathrm{C}=\mathrm{C} / \mathrm{C}=\mathrm{C}(/[\mathrm{F}]) \mathrm{C}=2) \mathrm{C}(=\mathrm{O}) \mathrm{N}[ \\
\mathrm{C} @ @]([\mathrm{H}])(\mathrm{C} / \mathrm{C} 3=\mathrm{C} / \mathrm{C}=\mathrm{CC}=\mathrm{C} 3)[\mathrm{C} @ @](\mathrm{O})([\mathrm{H}]) \mathrm{CN}(\mathrm{C} / \mathrm{C} 4= \\
\mathrm{C} / \mathrm{C}=\mathrm{C}[\mathrm{S}] 4)[\mathrm{S}](=\mathrm{O})(=\mathrm{O}) \mathrm{C} 5=\mathrm{CC}=\mathrm{CC}(=\mathrm{C} 5) \mathrm{OC}\end{array}$ \\
\hline H81 & $*$ & 3.599 & 3.366 & 72.722 & 3.251 & 82.770 & $\begin{array}{l}\mathrm{O}=\mathrm{C} 1 \mathrm{O}[\mathrm{C} @ @]([\mathrm{H}])(\mathrm{CN} 1 \mathrm{C}=2 \mathrm{C}=\mathrm{CC}=\mathrm{CC}=2) \mathrm{C}(=\mathrm{O}) \mathrm{N}[\mathrm{C} @ @ \\
]([\mathrm{H}])(\mathrm{C} / \mathrm{C} 3=\mathrm{C} / \mathrm{C}=\mathrm{CC}=\mathrm{C} 3)[\mathrm{C} @ @](\mathrm{O})([\mathrm{H}]) \mathrm{CN}(\mathrm{CC}(\mathrm{C})(\mathrm{C})[\mathrm{H}] \\
)[\mathrm{S}](=\mathrm{O})(=\mathrm{O}) \mathrm{C} 4=\mathrm{CC}=\mathrm{C}(\mathrm{C}=\mathrm{C} 4) \mathrm{CO}\end{array}$ \\
\hline H85 & $*$ & 3.777 & 4.234 & 91.478 & 3.389 & 86.293 & $\begin{array}{l}\mathrm{O}=\mathrm{C} 1 \mathrm{O}[\mathrm{C} @ @]([\mathrm{H}])(\mathrm{CN} 1 \mathrm{C}=2 \mathrm{C}=\mathrm{CC}([\mathrm{F}])=\mathrm{CC}=2) \mathrm{C}(=\mathrm{O}) \mathrm{N}[\mathrm{C} \\
@ @]([\mathrm{H}])(\mathrm{C} / \mathrm{C} 3=\mathrm{C} / \mathrm{C}=\mathrm{CC}=\mathrm{C} 3)[\mathrm{C} @ @](\mathrm{O})([\mathrm{H}]) \mathrm{CN}(\mathrm{CC}(\mathrm{C})( \\
\mathrm{C})[\mathrm{H}])[\mathrm{S}](=\mathrm{O})(=\mathrm{O}) \mathrm{C} 5=\mathrm{CC}=\mathrm{C} 4 \mathrm{OCOC} 4=\mathrm{C} 5\end{array}$ \\
\hline H97 & $*$ & 3.622 & 4.070 & 87.937 & 4.116 & 104.797 & $\begin{array}{l}\mathrm{O}=\mathrm{C} 1 \mathrm{O}[\mathrm{C} @ @]([\mathrm{H}])(\mathrm{CN} 1 \mathrm{C}=2 \mathrm{C}=\mathrm{CC}=\mathrm{C}(\mathrm{C}=2) \mathrm{OC}([\mathrm{F}])([\mathrm{F}])[\mathrm{F} \\
]) \mathrm{C}(=\mathrm{O}) \mathrm{N}[\mathrm{C} @ @]([\mathrm{H}])(\mathrm{C} / \mathrm{C} 3=\mathrm{C} / \mathrm{C}=\mathrm{CC}=\mathrm{C} 3)[\mathrm{C} @ @](\mathrm{O})([\mathrm{H}]) \\
\mathrm{CN}(\mathrm{CC}(\mathrm{C})(\mathrm{C})[\mathrm{H}])[\mathrm{S}](=\mathrm{O})(=\mathrm{O}) \mathrm{C} 5=\mathrm{CC}=\mathrm{C} 4 \mathrm{OCOC} 4=\mathrm{C} 5\end{array}$ \\
\hline H110 & $*$ & 3.914 & 4.440 & 95.940 & 4.107 & 104.555 & $\begin{array}{l}\mathrm{O}=\mathrm{C} 1 \mathrm{O}[\mathrm{C} @ @]([\mathrm{H}])(\mathrm{CN} 1 \mathrm{C}=2 \mathrm{C}=\mathrm{CC}=\mathrm{C}(\mathrm{C}=2) \mathrm{NC}(=\mathrm{O}) \mathrm{C}) \mathrm{C}(= \\
\mathrm{O}) \mathrm{N}[\mathrm{C} @ @]([\mathrm{H}])(\mathrm{C} / \mathrm{C} 3=\mathrm{C} / \mathrm{C}=\mathrm{CC}=\mathrm{C} 3)[\mathrm{C} @ @](\mathrm{O})([\mathrm{H}]) \mathrm{CN}(\mathrm{C} \\
\mathrm{C}(\mathrm{C})(\mathrm{C})[\mathrm{H}])[\mathrm{S}](=\mathrm{O})(=\mathrm{O}) \mathrm{C} 4=\mathrm{CC}=\mathrm{C}(\mathrm{C}=\mathrm{C} 4) \mathrm{OC}\end{array}$ \\
\hline H124 & $*$ & 3.788 & 3.769 & 81.446 & 4.380 & 111.506 & $\begin{array}{l}\mathrm{O}=\mathrm{C} 1 \mathrm{O}[\mathrm{C} @ @]((\mathrm{H}])(\mathrm{CN} 1 \mathrm{C}=2 \mathrm{C}=\mathrm{CC}=\mathrm{C}(\mathrm{C}=2) \mathrm{NC}(=\mathrm{O}) \mathrm{OC}) \mathrm{C}( \\
=\mathrm{O}) \mathrm{N}[\mathrm{C} @ @]([\mathrm{H}])(\mathrm{C} / \mathrm{C} 3=\mathrm{C} / \mathrm{C}=\mathrm{CC}=\mathrm{C} 3)[\mathrm{C} @ @](\mathrm{O})([\mathrm{H}]) \mathrm{CN}( \\
\mathrm{CC}(\mathrm{C})(\mathrm{C})[\mathrm{H}])[\mathrm{S}](=\mathrm{O})(=\mathrm{O}) \mathrm{C} 4=\mathrm{CC}=\mathrm{C}(\mathrm{C}=\mathrm{C} 4) \mathrm{OC}\end{array}$ \\
\hline H127 & $*$ & 3.866 & 3.410 & 73.685 & 3.840 & 97.765 & $\begin{array}{l}\mathrm{O}=\mathrm{C} 1 \mathrm{O}[\mathrm{C} @ @]([\mathrm{H}])(\mathrm{CN} 1 \mathrm{C}=2 \mathrm{C}=\mathrm{CC}=\mathrm{CC}=2 \mathrm{C}([\mathrm{F}])([\mathrm{F}])[\mathrm{F}]) \mathrm{C} \\
(=\mathrm{O}) \mathrm{N}[\mathrm{C} @ @]([\mathrm{H}])(\mathrm{C} / \mathrm{C} 3=\mathrm{C} / \mathrm{C}=\mathrm{CC}=\mathrm{C} 3)[\mathrm{C} @ @](\mathrm{O})([\mathrm{H}]) \mathrm{CN} \\
(\mathrm{CC}(\mathrm{C})(\mathrm{C})[\mathrm{H}])[\mathrm{S}](=\mathrm{O})(=\mathrm{O}) \mathrm{C} 4=\mathrm{C} / \mathrm{C}=\mathrm{C}(/ \mathrm{N}) \mathrm{C}=\mathrm{C} 4\end{array}$ \\
\hline
\end{tabular}


${ }^{1}$ Experimental $p K_{I} ;{ }^{2}$ Calculated $p K_{i}$

Table S4: Y-randomization test of Split 1 (Models 1 and 2)

Model 1

$\begin{array}{ccccccc} & \text { Training } & \text { Calibration } & \text { Test } & \text { Training } & \text { Calibration } & \text { Test } \\ \mathrm{n} & 70 & 20 & 21 & 70 & 20 & 21 \\ \text { Original } & 0.837 & 0.942 & 0.799 & 0.811 & 0.950 & 0.806 \\ 1 & 0.006 & 0.001 & 0.004 & 0.062 & 0.000 & 0.088 \\ 2 & 0.029 & 0.001 & 0.007 & 0.028 & 0.076 & 0.003 \\ 3 & 0.003 & 0.000 & 0.002 & 0.000 & 0.021 & 0.024 \\ 4 & 0.049 & 0.001 & 0.003 & 0.050 & 0.025 & 0.121 \\ 5 & 0.012 & 0.271 & 0.005 & 0.002 & 0.097 & 0.000 \\ 6 & 0.006 & 0.060 & 0.006 & 0.055 & 0.009 & 0.026 \\ 7 & 0.011 & 0.023 & 0.009 & 0.003 & 0.098 & 0.007 \\ 8 & 0.008 & 0.362 & 0.021 & 0.014 & 0.003 & 0.001 \\ 9 & 0.011 & 0.073 & 0.016 & 0.004 & 0.025 & 0.063 \\ 10 & 0.000 & 0.017 & 0.103 & 0.024 & 0.001 & 0.106 \\ R_{r}^{2} & 0.013 & 0.081 & 0.018 & 0.024 & 0.036 & 0.044 \\ { }^{2} \boldsymbol{R}_{p}^{2} & \mathbf{0 . 8 2 3} & \mathbf{0 . 9 0 7} & \mathbf{0 . 7 8 2} & \mathbf{0 . 8 2 4} & \mathbf{0 . 9 3 7} & \mathbf{0 . 7 9 6}\end{array}$


Table S5: Y-randomization test of Split 2 (Models 3 and 4)

$$
\text { Model } 3
$$

Training Calibration

72

$\mathrm{n}$

Original

1

2

3

4

5

6

6

7

8

9

10

$R_{r}^{2}$

${ }^{C} \boldsymbol{R}_{p}^{2}$

0.843

20

0.947

0.071

0.130

0.006

0.071

0.005

0.029

0.216

0.147

0.036

0.161

0.087

0.903

0.830

Test

Training

19

0.781

0.001

0.192

0.007

0.002

0.011

0.002

0.010

0.068

0.004

0.000

0.030

0.766

Model 4

Calibration

20

19

0.841

0.941

0.784

0.000

0.006

0.001

0.021

0.051

0.107

0.031

0.100

0.012

0.002

0.006

0.201

0.002

0.001

0.022

0.006

0.005

0.059

0.015

0.232

0.021

0.012

0.008

0.127

0.030

0.023

0.006

0.028

0.000

0.010

0.015

0.043

0.057

0.834

0.919

0.811 
Table S6: Y-randomization test of Split 3 (Models 5 and 6)

$$
\text { Model } 5
$$

Training Calibration Test

$\mathrm{n}$

Original

1

2

3

4

5

6

7

8

9

10

$R_{r}^{2}$

${ }^{C} \boldsymbol{R}_{p}^{2}$

0.829

0.945

0.001

0.008

0.001

0.017

0.000

0.003

0.001

0.099

0.005

0.007

0.203

0.004

0.040

0.001

0.056

0.002

0.002

0.010

0.012

0.004

0.045

0.827

0.923

19

Training

Model 6

Calibration

20

19

0.804

0.830

0.945

0.773

0.033

0.019

0.001

0.016

0.024

0.001

0.006

0.091

0.029

0.004

0.037

0.001

0.074

0.001

0.000

0.013

0.005

0.003

0.048

0.129

0.026

0.030

0.032

0.000

0.076

0.000

0.062

0.083

0.001

0.000

0.037

0.154

0.075

0.011

0.005

0.006

0.008

0.030

0.025

0.006

0.035

0.010

0.025

0.050

0.787

0.825

0.933

0.748 